Nova Southeastern University

Nova Southeastern University

\title{
Impact of Physical Therapist Attitudes and Beliefs on the Outcomes of Patients with Low Back Pain
}

\author{
Adam P. Rufa \\ Nova Southeastern University
}

Follow this and additional works at: https://nsuworks.nova.edu/hpd_pt_stuetd

Part of the Physical Therapy Commons

\begin{abstract}
All rights reserved. This publication is intended for use solely by faculty, students, and staff of Nova Southeastern University. No part of this publication may be reproduced, distributed, or transmitted in any form or by any means, now known or later developed, including but not limited to photocopying, recording, or other electronic or mechanical methods, without the prior written permission of the author or the publisher.
\end{abstract}

\section{NSUWorks Citation}

Adam P. Rufa. 2020. Impact of Physical Therapist Attitudes and Beliefs on the Outcomes of Patients with Low Back Pain. Doctoral dissertation. Nova Southeastern University. Retrieved from NSUWorks, College of Health Care Sciences - Physical Therapy Department. (185) https://nsuworks.nova.edu/hpd_pt_stuetd/185.

This Dissertation is brought to you by the Department of Physical Therapy at NSUWorks. It has been accepted for inclusion in Department of Physical Therapy Student Theses, Dissertations and Capstones by an authorized administrator of NSUWorks. For more information, please contact nsuworks@nova.edu. 


\title{
The Impact of Physical Therapist Attitudes and Beliefs on the Outcomes of Patients with Low Back Pain.
}

\author{
by \\ Adam P. Rufa \\ A dissertation submitted in partial fulfillment of the requirements for the degree of \\ Doctor of Philosophy \\ March 27, 2020
}

Nova Southeastern University

Dr. Pallavi Patel College of Health Care Sciences

Department of Physical Therapy 


\section{Approval/Signature Page}

We hereby certify that this dissertation, submitted by Adam Rufa, conforms to acceptable standards and is fully adequate in scope and quality to fulfill the dissertation requirement for the degree of Doctor of Philosophy in Physical Therapy.

Dr. Joshua Cleland, PT, PhD, FAPTA

Date

Chairperson of Dissertation Committee

Dr. Morey Kolber, PT, PhD, OCS

Member, Dissertation Committee

Dr. Jason Rodeghero PT, PhD, OCS, FAAOMPT

Member, Dissertation Committee

Date

Approved:

Dr. M. Samuel Cheng, PT, MS, ScD

Date

Director, Physical Therapy PhD Program

Dr. Shari Rone-Adams, PT, MHSA, DBA

Chair, Department of Physical Therapy

Dr. Stanley H. Wilson, PT, EdD, CEAS

Date

Dean and Associate Professor 


\section{Abstract}

Introduction: Physical therapist attitudes and beliefs about low back pain (LBP) have been shown to influence patient beliefs and affect clinician behavior. The purpose of this project was to investigate physical therapist attitudes and beliefs about LBP, identify factors that influence those beliefs, and determine if attitudes and beliefs have an impact on patient outcomes. Methods: This study was a retrospective cohort design that included a survey of physical therapists and the collection of patient outcomes from Focus on Therapeutic Outcomes, Inc. (FOTO). Attitudes and beliefs were measured using the Health Care Providers' Pain and Impairment Relationship Scale (HC-PAIRS) and the Pain Attitudes and Beliefs Scale for Physiotherapists (PABS-PT). Outcomes were measured using Computerized Lumbar Functional Scale change scores (CLFS), CLFS residual scores, number of visits, and Fear Avoidance Beliefs Scale physical activity subscale (FABQpa).

Results: Complete attitudes and beliefs scales were collected from 140 physical therapists. PABS-BM and PABS-BPS scores were predicted by a model that included age between 18 and 34, board certification, and NPQ scores. A multiple variate model could not be developed for HC-PAIRS scores, as NPQ score was the only significant predictor. A linear model containing HCPAIRS scores and change in FABQpa scores predicted $16.1 \%$ of the variability in CLFS scores and $12.8 \%$ of the variability in the number of visits. HC-PAIRS was a univariate logistic predictor for a greater than expected CLFS change for the 10 patient cut-off sample. Receiver operating characteristic (ROC) curve identified an HC-PAIRS cut-off score of 30.50. This score had a sensitivity of .564 and specificity of .641. Scores on the PABS-PT scale were not multivariate predictors of any outcome measure. 
Conclusion: Several factors predicted LBP-related attitudes and beliefs, with the most consistent predictor being knowledge of current pain science. The LBP attitudes and beliefs of physical therapists were not consistent predictors of outcomes. HC-PAIRS scores were found to be related to outcomes in 8 of the 33 performed analyses; however, this relationship was not in the predicted direction. Physical therapists who believed there was a stronger relationship between pain and disability had better outcomes. 


\section{Acknowledgments}

I would like to extend my sincere thank you to the many people who have supported me through this process. Without their active guidance, cooperation, and encouragement I would not have been successful in this endeavor.

I would first like to express my deepest appreciation to the chair of my committee, Dr. Josh Cleland. Despite his extremely busy schedule, he was always willing to provide timely advice and guidance. He helped me move forward with this project in a rigorous and pragmatic way.

I would also like to thank the other members of my committee, Dr. Morey Kolber and Dr. Jason Rodeghero. Their guidance was invaluable and they were instrumental in pushing me to think more deeply about the concepts covered in this project.

This dissertation would not have been successful without the strong support of my colleagues at SUNY Upstate Medical University. They helped me find time to work on the project and they provided expert advice on statistics, design, and logistics. Without their support and guidance, this project would not have been successful.

Lastly, I need to thank my family. My wife, Dr. Jennifer Rufa, not only supported me with love and time, she spent many hours reading over drafts and editing. Without her assistance and understanding, this project would not have been completed. I am also extremely thankful for the love and support of my three children, Amelia, Oliver, and Will. Their joy, brilliance, humor, love, and understanding helped me push through the many late $n$ 


\section{$\underline{\text { Table of Contents }}$}

Title Page

Approval/Signature Page ............................................................................ii

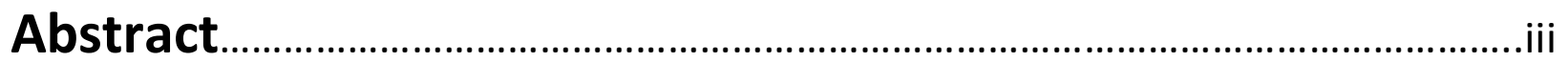

Acknowledgements............................................................................................

Table of Contents................................................................................................

\section{Chapter 1: Introduction}

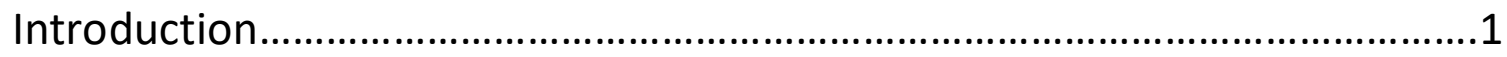

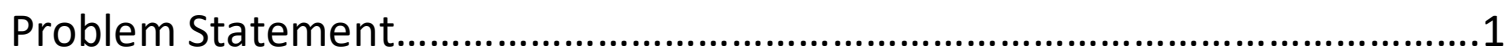

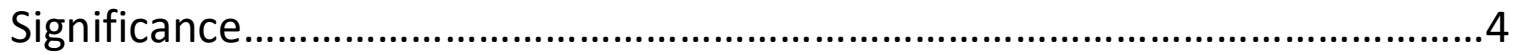

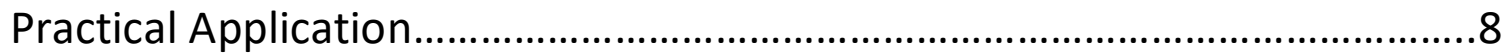

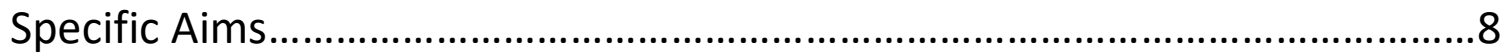

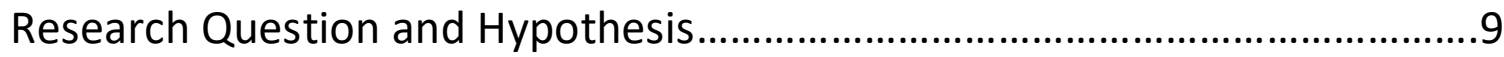

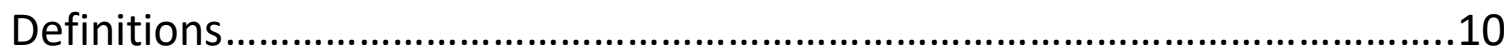

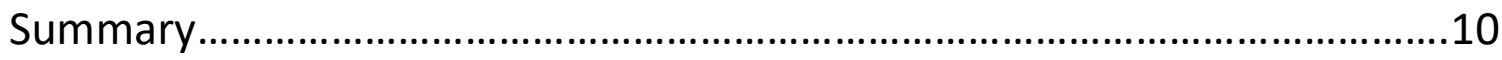

\section{Chapter 2: Literature Review}

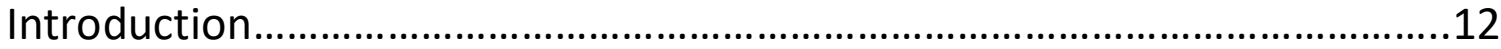

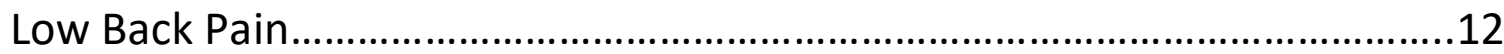

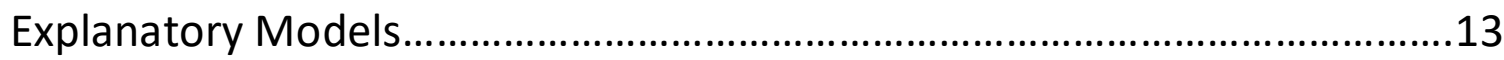

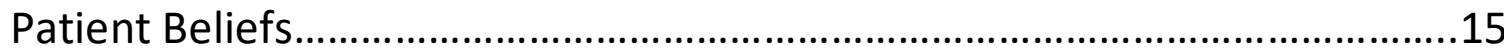

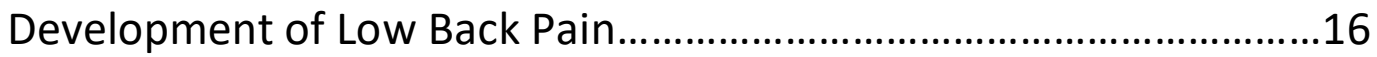

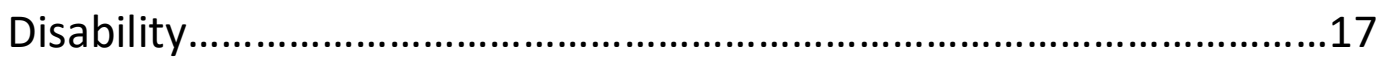

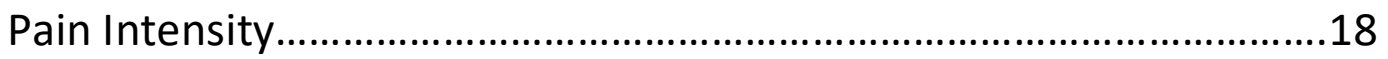

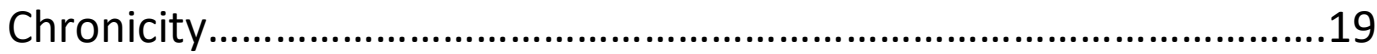

Treatment Success and Prognosis........................................................20

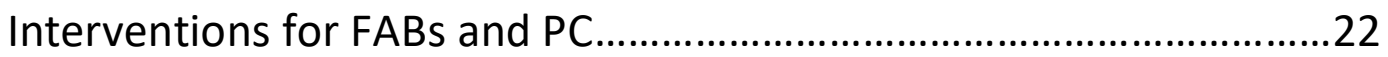

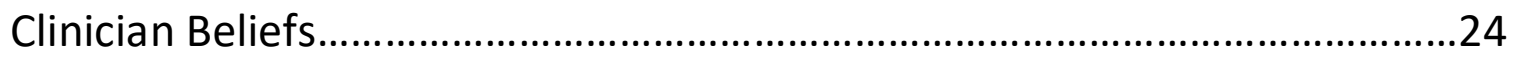

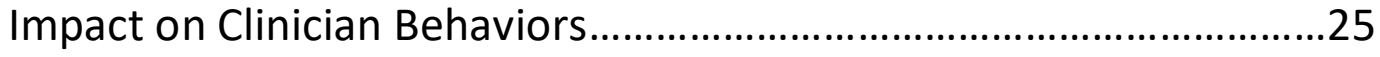

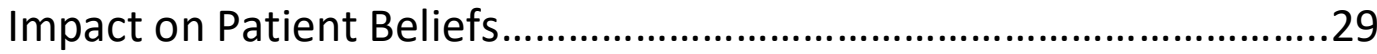

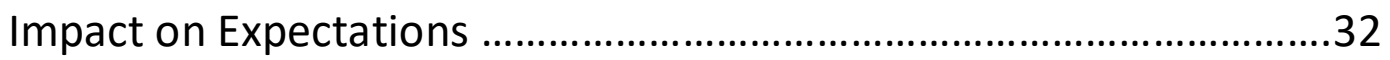

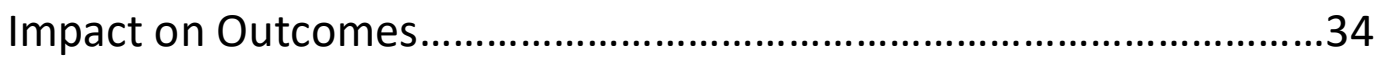

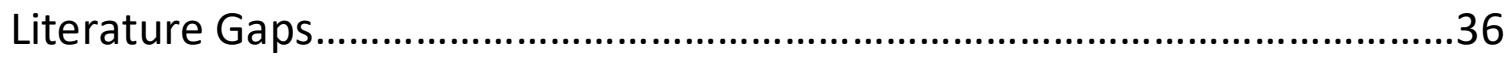

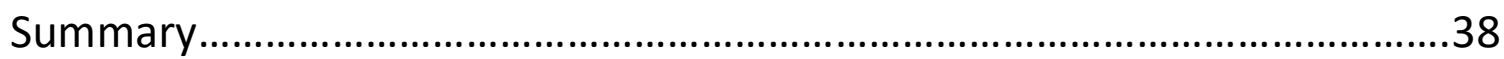

\section{Chapter 3: Methodology}




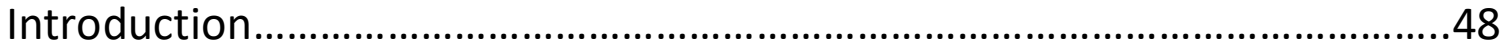

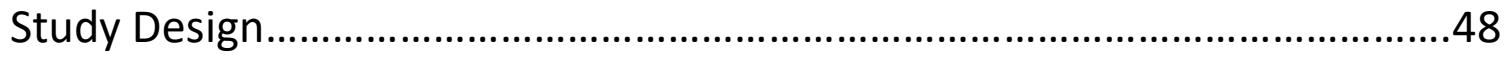

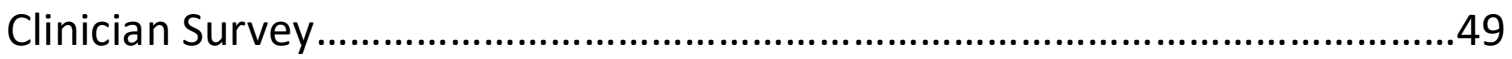

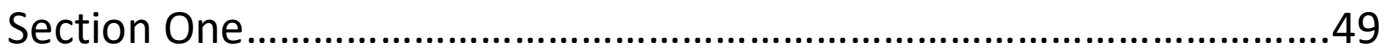

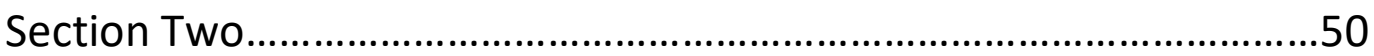

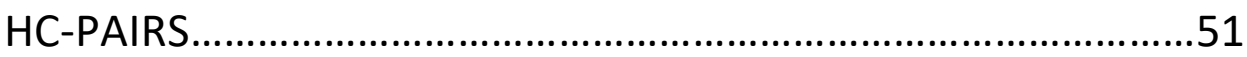

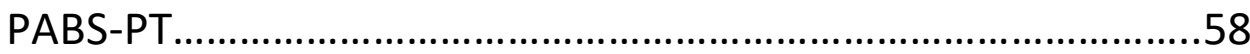

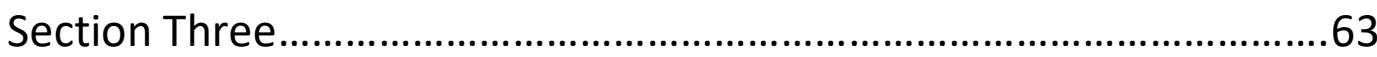

NPQ

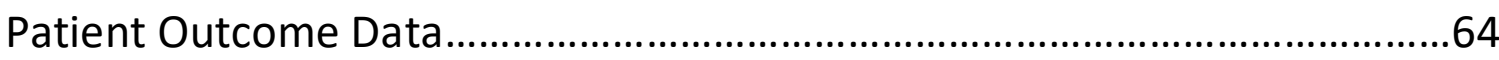

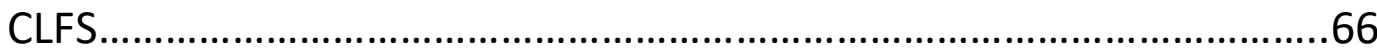

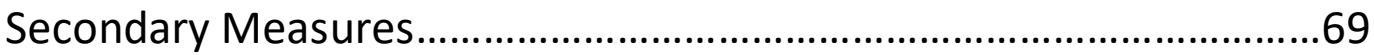

\section{Chapter 4: Results}

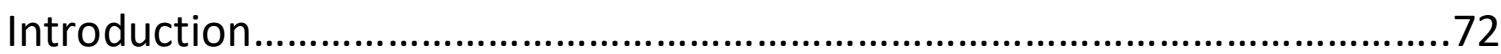

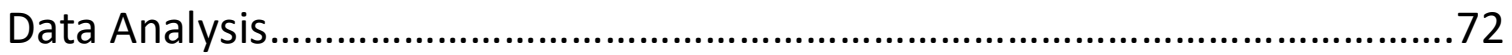

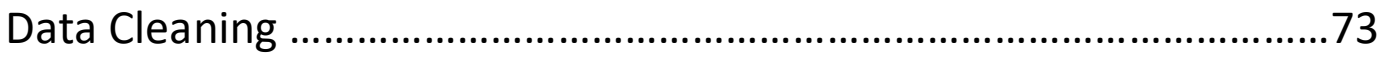

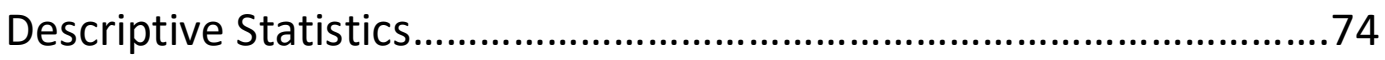

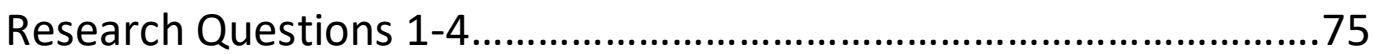

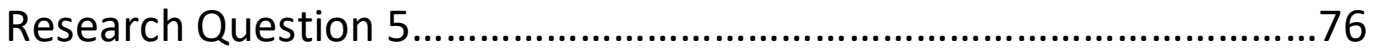

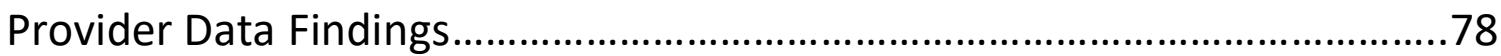

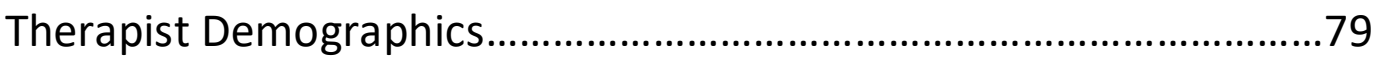

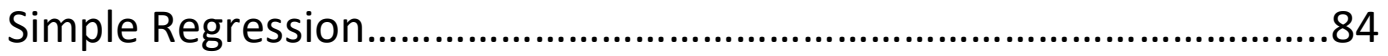

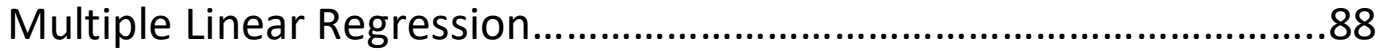

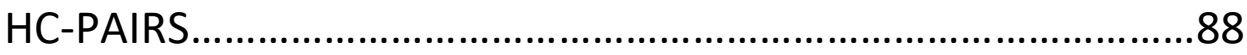

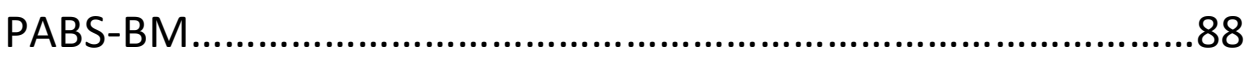

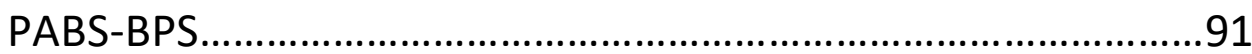

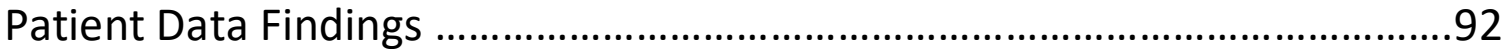

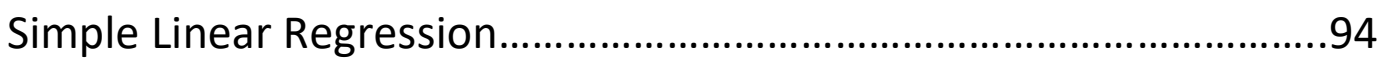

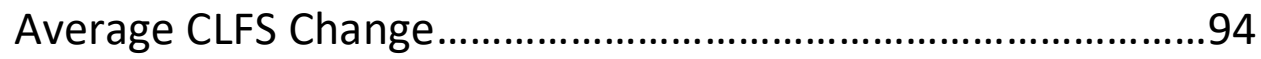

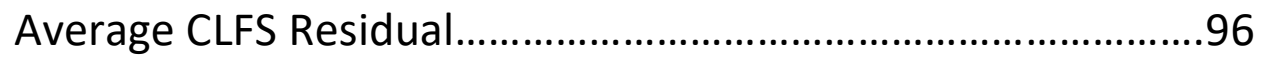

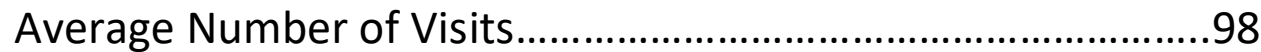

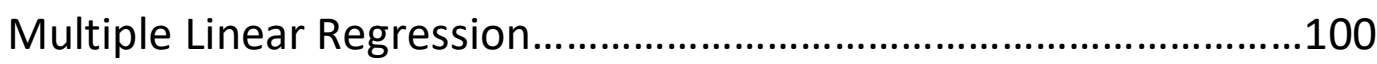

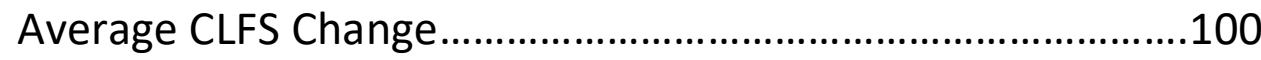

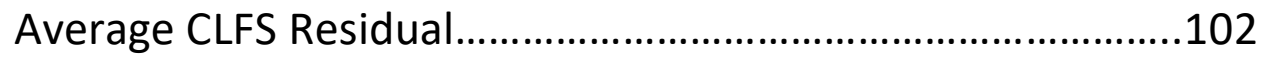

Average Number of Visits.....................................................105

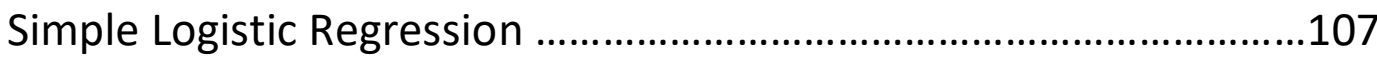

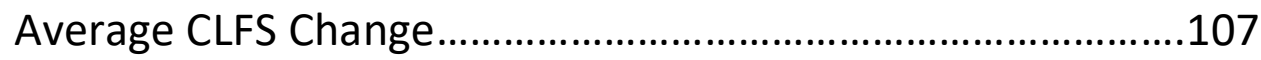


Average CLFS Residual......................................................112

Multiple Logistic Regression ...........................................................116

Average CLFS Change .........................................................116

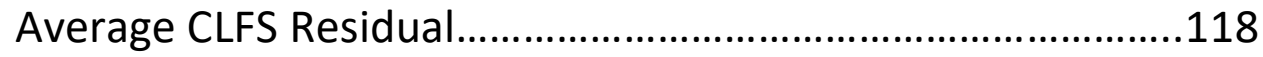

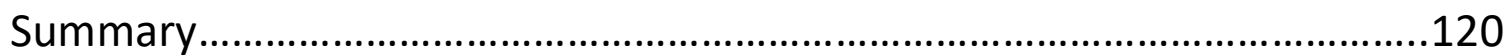

\section{Chapter 5: Discussion}

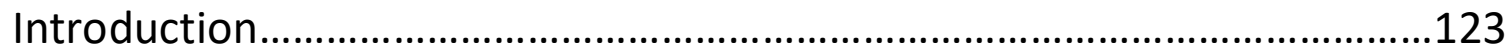

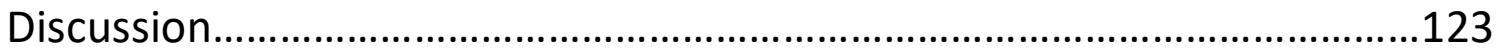

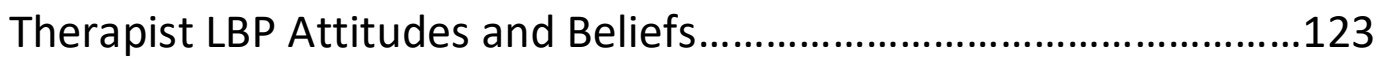

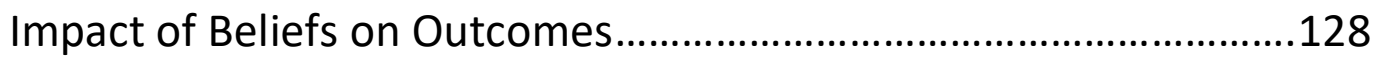

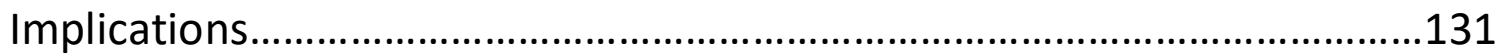

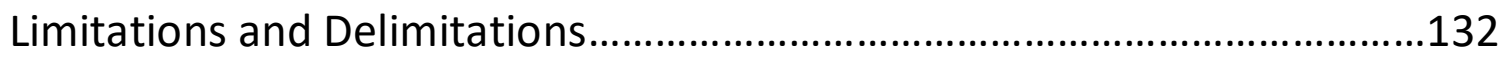

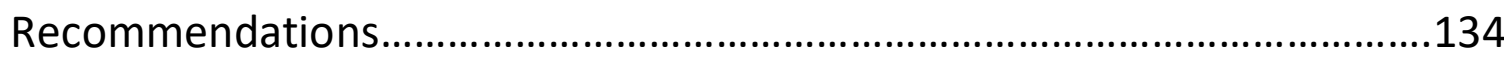

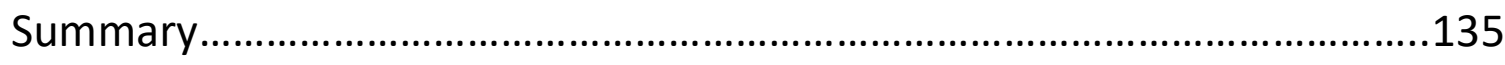

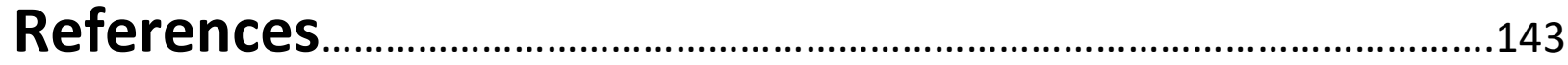

\section{Appendix}

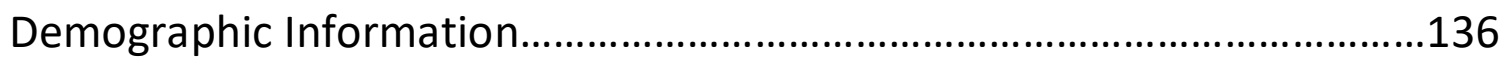

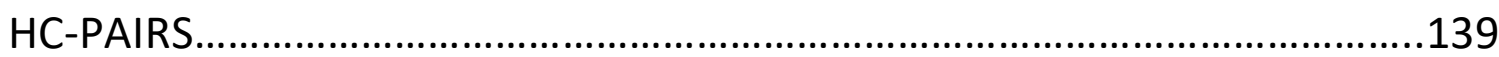

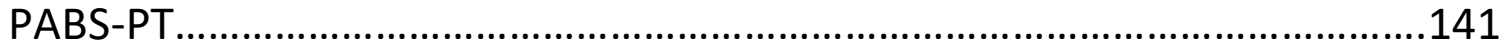

NPQ 


\section{List of Tables}

Table 1

(Impact of Attitudes and Beliefs on Clinician Behavior)

Table 2

(Impact of Attitudes and Beliefs on Patient Beliefs)

Table 3

(Impact of Attitudes and Beliefs on Patient Outcomes)

Table 4

(Mean belief and knowledge scores by demographic categories)

Table 5

(Scores for beliefs and knowledge questionnaires)

Table 6.

(Simple linear regression of beliefs scores by physical therapist demographics)

Table 7 ....

(Multiple regression modeling to predict HC-PAIRS scores)

Table 8

(Multiple regression modeling to predict PABS-BM scores)

Table 9

(Multiple regression modeling to predict PABS-BPS scores)

Table 10

(Patient data, physical therapist beliefs, and knowledge by sample)

Table 11

(Simple linear regression of average CLFS change scores)

Table 12

(Simple linear regression of average CLFS residual scores)

Table 13

(Simple linear regression of average number of visits)

Table 14

(Hierarchical multiple linear regression of average CLFS change)

Table 15

(Hierarchical multiple linear regression for average CLFS residual scores)

Table 16

(Hierarchical multiple linear regression of average number of visits)

Table 17

(Simple logistic regression of average CLFS scores)

Table 18

(Simple logistic regression of average CLFS residual)

Table 19.

(Receiver operating characteristic curve for HC-PAIRS ability to predict CLFS residual) 


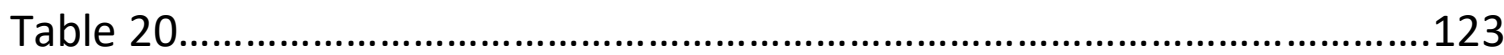

Receiver operating characteristic curve coordinates for HC-PAIRS ability to predict CLFS residual)

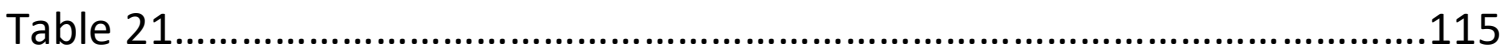

(Multiple logistic regression for CLFS change)

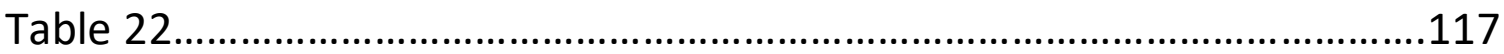

(Multiple logistic regression for average CLFS residual) 


\section{List of Figures}

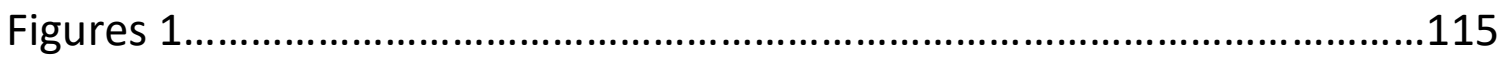

(Receiver operating characteristic curve for HC-PAIRS ability to predict CLFS residual) 


\section{Chapter 1: Introduction}

\section{Introduction}

This chapter is an introduction to the dissertation. It will discuss the problem that was addressed, describe the significance of the dissertation, outline the research questions and hypotheses, and define important terms that are used in the dissertation. The chapter also gives a brief overview of the current literature that demonstrates the significance of the problem addressed in this paper.

\section{Problem Statement}

Low back pain (LBP) is a complex disorder that places a major burden on individuals and society. ${ }^{1,2}$ Bio-pathoanatomical (BPA) models of LBP can explain some aspects of the disorder; however, they have struggled to produce highly effective treatment strategies. ${ }^{3-6}$ In hopes of improving on BPA models, more comprehensive explanations, which include BPA, psychological and social factors, have been proposed. ${ }^{7}$ These models consider the impact that cognitive factors have on the perception of pain and on the severity of disability. Models such as the biopsychosocial model (BPS), cognitive behavioral theory (CBT), and the fear-avoidance beliefs (FAB) model, stress that subjective factors, such as patient beliefs, have an influence on musculoskeletal conditions. Interventions aimed at influencing these subjective factors seem to have a positive influence on recovery. ${ }^{8}$ It has recently been proposed that similar cognitive factors in healthcare providers may have an influence on patients with musculoskeletal pain. ${ }^{9}$ For example, physical therapist attitudes and beliefs about LBP may influence the beliefs of 
their patients and may affect the advice and treatment physical therapists provide to patients. ${ }^{10,11}$

It is currently unclear, however, if physical therapist attitudes and beliefs about LBP have an influence on patient beliefs and/or if they have an impact on patient outcomes. If physical therapist LBP-related attitudes and beliefs do influence outcomes, it suggests that strategies to optimize physical therapist attitudes and beliefs should be developed.

LBP has an estimated global prevalence of $31 \%$ and is the number one cause of disability around the globe. ${ }^{1,2}$ The reported lifetime prevalence of LBP is variable and has been reported to be approximately $80 \% .{ }^{12-14}$ In 2010 , LBP accounted for 83 million disability-adjusted life years, making it the sixth greatest contributor to years lived with disability globally. ${ }^{2}$ Chronic low back pain (CLBP) is less prevalent but results in a significant burden on individuals and society. Studies examining the prevalence of CLBP throughout the world have reported ranges from 3.9\%-20\%. ${ }^{15}$ A 2010 internet-based study of 27,035 individuals from the United States (US) reported an $8.1 \%$ prevalence of LBP lasting greater than 6 months. ${ }^{16}$ LBP costs the US more than $\$ 100$ billion per year; $75 \%$ of that cost is incurred by fewer than $5 \%$ of individuals with CLBP. ${ }^{17}$ Patients with LBP comprise over $25 \%$ of all outpatient physical therapy visits, ${ }^{18}$ and physical therapy accounts for $17 \%$ of the direct costs of LBP. ${ }^{19}$

Despite the high prevalence of LBP and the large body of literature on the topic, rates of LBP continue to increase. ${ }^{17}$ As a result, researchers have searched for more comprehensive explanatory models that build on the large body of evidence, primarily examining BPA factors. Models such as the BPS model, CBT, and the FAB model expand on the BPA model by 
incorporating cognitive elements. The BPS model was first introduced by Engel in 1977 and suggests that pain and disability are influenced by biological, psychological, and social factors. ${ }^{20,21}$ Cognitive behavioral theories of pain were popularized in the early 1980 s and built on the work of behavioral psychologists. ${ }^{22}$ These theories focused on the interplay between thoughts, emotions, and behaviors. Interventions focus on influencing thoughts and emotions in hopes of improving function and reducing disability. ${ }^{7}$ The FAB model suggests that anxiety, excessive pain-related fear, reduced physical activity due to avoidance, and societal factors can affect the amount and duration of pain, increase disability, and limit function. ${ }^{23,24}$ Although slightly different, these three models all build on the BPA model by adding cognitive factors, and they attempt to provide a more comprehensive understanding of chronic pain.

The importance of cognitive factors in individuals with LBP has been supported in the literature. Several studies have found that psychological risk factors have a modest influence on the development of LBP ${ }^{3,25-27}$ and increase the risk of developing CLBP. ${ }^{28,29}$ Picavet et al. surveyed 1,845 individuals with a 6-month follow-up and found that pain catastrophizing and kinesophobia were predictors of CLBP and associated with higher levels of pain levels. ${ }^{30}$ In two systematic reviews, Wertli et al. found that FABs and pain catastrophizing had an impact on work-related outcomes, such as time out of work. ${ }^{31,32}$ Cognitive factors are also associated with higher pain levels, increased disability, ${ }^{8,33-36}$ and treatment success. ${ }^{31,37-41}$ Changes in FABs are associated with reduced pain and improved disability, ${ }^{8}$ and interventions targeting negative beliefs about LBP have been found to decrease pain, improve physical performance, lessen anxiety and depression, and reduce healthcare utilization. ${ }^{42}$ 
With the growing appreciation of the importance of patient cognitive factors, several authors have suggested that clinician attitudes and beliefs about LBP may have an impact on patient outcomes. ${ }^{10,11,43-45}$ Clinicians whose beliefs are more BPA-oriented may provide advice and recommendations that limit physical activity and promote the development of psychosocial risk factors. ${ }^{46,47}$ Although several studies have been performed regarding physical therapist attitudes and beliefs about LBP, the beliefs of physical therapists in the US are unclear and it is unknown if these attitudes and beliefs have an impact on patient outcomes. ${ }^{46,48}$ The purpose of this proposed project is to investigate physical therapist attitudes and beliefs about LBP, identify factors that influence those beliefs, and determine if attitudes and beliefs have an impact on patient outcomes.

\section{Significance}

Approximately $80 \%$ of individuals will have LBP in their lifetime. Of those individuals, 70 to 90 percent will recover in 6 weeks $^{49}$ and approximately $10 \%$ will go on to develop CLBP. ${ }^{50}$ Although most individuals with LBP recover, the majority of those individuals will have a recurrence of pain within a year. ${ }^{43,51}$ Despite significant research, the rates of LBP in our society remain high, the cost continues to increase ${ }^{17}$, and the best treatment approach is unclear. ${ }^{52,53}$ There is a growing body of evidence suggesting that cognitive factors may have an important influence on LBP. ${ }^{26,29}$ It has also been suggested that clinicians' thoughts and beliefs about LBP have an influence on recovery. ${ }^{46,54}$ Understanding this relationship could lead to improved outcomes for patients with LBP. 
A clinician's LPB-related attitudes and beliefs may influence the course of the disorder in several ways. One potential mechanism for this effect is the influence that clinician attitudes and beliefs have on the beliefs of their patients. A 2012 systematic review, including 17 articles from 8 countries, found strong evidence that patient beliefs about LBP were associated with the beliefs of their health care professionals. ${ }^{55} \mathrm{~A}$ more recent qualitative study involving New Zealanders with LBP found that healthcare professionals had the largest impact on patient beliefs. ${ }^{56}$ Participants in this study reported feeling uncertain about why they had pain and what they should do about it. These participants sought information from several sources, such as family, friends, and the internet, but they had the greatest confidence in the information provided to them by healthcare professionals. Clinicians who provided information and advice that focused on BPA factors, such as muscles and posture, and who suggested that certain activities be avoided, caused guilt, frustration, and worry in patients. ${ }^{56}$ This suggests that a clinician who believes movement is dangerous and damaging to the back may talk and act in a way that increases a patient's fear and reduces their willingness to move. ${ }^{56-58}$ These cliniciandriven changes in fear avoidance, kinesophobia, and pain catastrophizing could lead to higher pain levels, increased disability, and an overall worse outcome for patients. $8,32,36,39,59,60$

Clinician attitudes and beliefs about LBP influence the advice they provide to patients and may affect the treatment choices they make. Several studies have found that clinicians who are more biomedically-oriented and who have lower movement and functional expectations advise patients to move less and limit work activities. ${ }^{46,54,61-63}$ For example, Houben et al. measured the beliefs of 295 physical therapists in the Netherlands. They found that physical therapists with a biomedical orientation saw activities as being more harmful, and when 
provided with patient vignettes, were more likely to recommend activity limitations and work avoidance. ${ }^{46} \mathrm{~A}$ qualitative study of 6 physical therapists and 12 patients reported that patient education and treatment decisions were closely related to the physical therapists' beliefs. The therapists involved in the study had strong BPA beliefs such has pain being caused by poor muscle control, stiffness, obesity, job duties, and disc injury. Treatment was aimed at correcting the BPA findings and prognosis was determined based on the ability to change the BPA factors. ${ }^{47}$ These studies suggest that greater BPA-oriented beliefs increase the likelihood that a physical therapist will provide advice and treatment that is not aligned with current guidelines. ${ }^{64}$ This could lead to worse outcomes or reduced clinical efficiency for patients treated by physical therapists who have BPA-oriented beliefs.

Physical therapist attitudes and beliefs about LBP also have an influence on the functional expectations physical therapists have for patients. ${ }^{47}$ This phenomenon was first described by Rosenthal in 1963 and has been called "the expectancy effect." 65 Rosenthal observed that the expectations of an examiner had a significant impact on the speed rats could complete a maze. ${ }^{65}$ When researchers thought they were testing highly intelligent rats, those rats finished the maze sooner than identical rats that were viewed as having normal intelligence. It is possible that this effect could carry over to the clinical setting, resulting in changes in outcomes based solely on the expectations of the healthcare provider. The clinical application of this effect was demonstrated in a study by Galer et al.; they found a significant correlation between a physicians' expectation that an injection would be effective and the actual change in pain after the injection. ${ }^{66}$ This effect has also been observed in physical therapy students during an isometric lifting task. Students with high fear-avoidance who were 
tested on a lifting task by other students with high FAB had a $14.4 \mathrm{~kg}$ reduction in lifting capacity. ${ }^{9}$ The exact mechanism(s) of this effect is unclear; however, a lack of clinical equipoise could lead to subtle changes in a clinician's verbal and non-verbal communication which, in turn, could influence patient performance. This could lead to worse outcomes for patients with LBP who are treated by physical therapists with BPA-oriented attitudes and beliefs.

Several studies have been published which look at the attitudes and beliefs of physical therapists and how those beliefs affect decision making. These studies have provided a theoretical basis for how the beliefs of physical therapists could influence patient outcomes. The vast majority of these studies have been performed outside the US and it is unclear if the beliefs of physical therapists within the US are similar to those of other countries. It is also unclear if beliefs have a meaningful impact on patient outcomes. A 2011 Swedish study of 42 physical therapists and 266 patients found that patient outcomes did not change after physical therapists underwent an 8-week class focused on psychosocial prognostic factors. ${ }^{44}$ This may indicate that clinicians' beliefs do not have an impact on outcomes; however, the small sample size, questionable risk adjustment strategies, and focus on changes in physical therapist beliefs limit the conclusions that can be drawn from this study. Further investigation into the impact of physical therapist attitudes and beliefs on patient outcomes could help to determine if greater attention should be given to measuring and modifying the attitudes and beliefs of physical therapists. 


\section{Practical Application}

If the attitudes and beliefs of physical therapists about LBP have an impact on outcomes, then educational interventions targeting these beliefs may lead to improved patient recovery. Entry-level education and post-professional courses aimed at improving physical therapist attitudes and beliefs could help to ensure that patients obtain the best recovery possible. It may also be important to screen physical therapists and student physical therapists for high-risk beliefs and target interventions at those clinicians whose beliefs may lead to worse outcomes. The identification of factors that influence the attitudes and beliefs of physical therapists will assist in developing strategies to improve them. If physical therapist attitudes and beliefs do not impact the outcomes of patients with LBP, efforts to change those beliefs may be an inefficient use of time and resources.

\section{Specific Aims}

1. Describe the attitudes and beliefs of US physical therapists regarding low back pain.

2. Determine if physical therapist knowledge about the physiologic mechanisms of pain is aligned with current evidence.

3. Determine factors that influence physical therapist attitudes and beliefs regarding low back pain.

4. Determine if physical therapist attitudes and beliefs are associated with the outcomes of patients with low back pain. 


\section{Research Question and Hypothesis}

\section{Questions:}

1. What are the characteristics of the physical therapists who participated in the study?

2. What are the low back pain related attitudes and beliefs of US physical therapists?

3. Do physical therapists have pain science knowledge that is aligned with current evidence (as measured by the Revised Neurophysiology of Pain Questionnaire)?

4. What factors are associated with physical therapist attitudes and beliefs about low back pain?

5. Are physical therapist attitudes and beliefs associated with the outcomes of patients with low back pain?

\section{Research Hypothesis:}

H1 Physical therapist traits, such as a personal history of low back pain, a personal history of invasive interventions for low back pain, a lack of board certification or residency/fellowship training, and limited knowledge about pain mechanisms, will be associated with greater BPA-oriented attitudes and beliefs.

H2 Patients with low back pain treated by physical therapists with BPA-oriented beliefs will improve less than those treated by physical therapists with lower BPA-oriented beliefs. 


\section{$\underline{\text { Definitions }}$}

Back pain: An unpleasant sensory and emotional experience that occurs in an area from the first thoracic vertebrae to the sacrum or is associated with a disorder of those areas.

Low back pain: An unpleasant sensory and emotional experience that is located below the ribs and above the gluteal folds.

Bio-pathoanatomical model: An explanatory model that addresses the origins of back pain in terms of tissue and biomechanical pathology.

Biopsychosocial model: An explanatory model that focuses on the origins of back pain in terms of tissue and biomechanical pathology, social, psychological, and behavioral factors.

Attitudes and beliefs (about low back pain): The attitudes and beliefs of physical therapists about the connection between tissue damage, low back pain, and function.

\section{Summary}

LBP is a common problem that results in disability and financial burden. Despite significant scientific investigation, the cause of LBP remains unclear and there is a lack of highly effective treatment strategies. There has been a growing interest in investigating the influence of psychological and social factors on LBP. Studies have suggested that factors such as pain catastrophizing and fear avoidance can have an influence on the development and prognosis of LBP. More recently, it has been proposed that clinician attitudes and beliefs about the relationship between pain, tissue injury, and function may have an impact on the outcomes of 
patients with LBP. This dissertation will investigate the attitudes and beliefs of physical therapists and their influence on the outcomes of patients with LBP. 


\section{Chapter 2: Literature Review}

\section{Introduction}

This chapter includes a review of the concepts and literature pertaining to the impact of clinician attitudes and beliefs about low back pain (LBP). The chapter will start by describing LBP and detailing the impact this disorder has on individuals and society. The evolution of explanatory models for LBP from a pathoanatomical-dominated model to a broader biopsychosocial model will also be covered. Next, the negative impact of certain patientoriented beliefs will be discussed which will lead into a discussion of the potential impact of clinician LBP-related attitudes and beliefs have on patient outcomes. Finally, current gaps in the literature will be highlighted and the purpose of this dissertation will be explained.

\section{Low Back Pain}

Pain has been defined as "an unpleasant sensory and emotional experience associated with actual or potential tissue damage, or described in terms of such damage" by the International Association for the Study of Pain. ${ }^{67}$ The low back is not well defined as an anatomical region, but is traditionally associated with the lumbosacral spine. LBP can have various definitions and has been described as pain that is located below the ribs and above the gluteal folds. ${ }^{68}$ Lower back dysfunction may also result in symptoms that are felt in other areas of the body. These symptoms can be concurrent with LBP or arise independently. A common example is lower extremity referred pain that has an etiological origin in the LBP.

Classification of LBP in the literature is variable and includes methods based on duration of symptoms, location of symptoms, treatment response, pathoanatomical characteristics, or 
radiographic abnormalities. ${ }^{69-71}$ Symptom duration is one of the most commonly used classification systems in the literature and divides patients into three broad categories: acute, sub-acute, and chronic. Exact definitions can vary between studies, but, authors generally describe acute LBP as pain lasting less than 4 weeks, sub-acute LBP as pain lasting between 4 and 12 weeks, and chronic LBP as pain lasting greater than 12 weeks. ${ }^{64,71}$

LBP is the most common musculoskeletal disorder and is estimated to affect over 2 billion people globally. ${ }^{1,72}$ A 2016 article on the global impact of disease and injury found that LBP has remained the number one cause of disability over the last 25 years. ${ }^{73}$ The prevalence of LBP varies widely across studies and between countries, and the one-year prevalence has been reported to be as low as 7.0\% (Denmark) and as high as $76.0 \%$ (Germany). ${ }^{1}$ In the United States (US), studies have found the one-year prevalence of LBP to range from $10.3 \%{ }^{74}$ to $56 \%{ }^{75}$, with a lifetime prevalence up to $80 \% .^{1,72,76,77}$ Females have higher rates of LBP than males and LBP prevalence tends to have a curvilinear relationship with age. ${ }^{1}$ When looking at all LBP, prevalence is highest in middle age $;^{1,73,76}$ however, severe LBP tends to increase steadily with age. ${ }^{1}$ Chronic low back pain (CLBP) is less prevalent than acute or sub-acute LBP, with ranges from 3.9\%-20.0\% ${ }^{15}$ Although CLBP occurs less frequently, it accounts for $75 \%$ of the more than $\$ 100$ billion dollars spent on LBP each year in the US. ${ }^{17}$

\section{Explanatory Models}

Traditionally, LBP has been seen as a tissue-based disorder. ${ }^{20,78}$ Researchers and clinicians have focused on pathoanatomical sources of pain and dysfunction, such as discogenic pathology and degenerative changes. Studies investigating bio-pathoanatomical (BPA) influences on pain 
have improved our understanding of anatomical and physiological changes that may influence LBP; however, these factors have not fully explained the complex nature of the condition. ${ }^{4-6,79}$ For example, BPA models of LBP have not been able to adequately explain the disconnect between structural changes in the back and the heterogeneity of patient signs and symptoms. ${ }^{3,4,80}$ As a result, clinicians and researchers searched for broader explanatory models that could potentially lead to new areas of research and more effective interventions to prevent and treat LBP. In 1977, George Engle described the conflict between a medical model of disease and the needs of psychiatrists for a broader view of disease and disability. ${ }^{20}$ He felt that all of medicine was in a crisis due to an "adherence to a model of disease no longer adequate for the scientific tasks and social responsibilities of either medicine or psychiatry." ${ }^{20} \mathrm{He}$ argued that the medical model's assumption that disease can be completely captured by measurable bioanatomical factors was faulty, and that social, psychological, and behavioral factors should be considered. Engle proposed that medicine move away from a reductionist biomedical model and proposed the biopsychosocial model of disease (BPS). ${ }^{20}$ This model stresses that simply addressing BPA factors may not be sufficient to return patients to full health. Instead, health and healing are predicated on treatment of biological, psychological, social, and behavioral factors.

Around the same time that Engle was proposing his BPS model, Fordyce was describing behavioral-based interventions for treating patients with chronic pain. ${ }^{81-84}$ Fordyce suggested that pain behaviors were operant and influenced by environmental factors. For example, he described how activity tolerance in patients with pain is influenced by contextual factors, such as verbal reinforcement, attention, and social context. ${ }^{81}$ Since pain cannot be directly measured or observed, Fordyce suggested that interventions should focus on the emergent behaviors that 
were amenable to observation. Behaviors were said to initially be responses to actual or potential tissue damage; however, chronic pain was seen as a persistence of this behavior beyond the threat to the tissue. ${ }^{83}$ Behavioral-based interventions were not aimed at changing nociception, but were focused on influencing pain expressions such as disability and suffering. ${ }^{83}$

Gordon Waddell was one of the first practitioners to write about the use of BPS and behavioral models for the treatment of patients with LBP78,85 His 1987 article in Spine highlighted the apparent disconnect between the physical aspects of LBP and the resulting pain and disability. ${ }^{78}$ Waddell described a treatment approach that targeted psychological and behavioral aspects of the disorder. Since that article, there has been a growing interest in the impact of psychological and behavioral factors on low back pain. Guidelines now suggest that psychological factors be considered, ${ }^{64,86}$ and treatments to address psychological and behavioral aspects of LBP, such as Cognitive Behavioral Therapy (CBT), ${ }^{87}$ pain neuroscience education (PNE), ${ }^{42}$ and graded exposure ${ }^{88}$ have continued to grow in popularity.

\section{$\underline{\text { Patient Beliefs }}$}

In addition to more psychologically informed treatment, there has been an expanding body of literature, and growing appreciation for, the importance of patient beliefs in the examination and treatment of musculoskeletal conditions. Fear-avoidance beliefs (FABs) and pain catastrophizing (PC) are two of the most commonly studied topics in this area and may play an important role in the development, persistence, and treatment of LBP.

The fear-avoidance model was first described by Lethem in 1983 and suggests that a patient's attitudes and beliefs about LBP influence the development and continuation of pain 
and disability. ${ }^{24,89}$ Under this model, fear of pain can lead to either avoidance or confrontation behaviors. Patients who fall more on the confrontation side of the continuum have less disability because they continue to move and function despite pain. Those who demonstrate more avoidance behaviors reduce their physical activity and attempt to avoid movement and activity that does or may cause pain. ${ }^{24}$ The model predicts that avoidance behaviors will lead to increased pain, higher levels of disability, and a greater chance of developing chronic pain.

The concept of catastrophizing in medicine was first introduced in the 1950 s and was seen as an irrational belief about the severity of a condition or problem. ${ }^{90} \mathrm{PC}$ occurs when patients see their pain and/or resulting disability as greater than it actually is. This exaggerated negative belief about pain is thought to result in higher levels of pain, greater disability, and is likely related to FABs. ${ }^{91}$ Three dimensions of PC have been identified: rumination, magnification, and helplessness. ${ }^{92}$ Rumination is excessive thought and worry about pain, magnification is the perception that the condition is more serious than it is, and helplessness is the feeling that nothing can be done about the condition. In general, studies have supported the hypothesis that patient's beliefs about LBP have an impact on the condition. FABs and PC have been implicated as factors impacting the development of LBP, ${ }^{26}$ the level of disability, ${ }^{93}$ pain intensity, ${ }^{94}$ conversion to chronic pain, ${ }^{28}$ treatment success, ${ }^{95}$ and overall prognosis. ${ }^{96}$

\section{Development of Low Back Pain}

There have been several studies that implicate FABs and PC as risk factors for the development of LBP in asymptomatic individuals. In a prospective study of 415 participants without spine pain, Linton et al found that those who scored above the median (9 points) for 
FABs were two times more likely to develop back pain and at a 1.7 times greater risk of having lower physical functioning in the subsequent year. ${ }^{27}$ In a similar study, Picavet et al. found that high levels of FABs and PC predicted the development of LBP in a pain-free cohort over a 6 month period. ${ }^{30}$ In a systematic review, Linton identified 37 prospective studies that investigated risk factors for the development of neck and back pain. Psychological factors, including FABs, were consistent risk factors for the development of LBP. Four studies investigated the impact of FABs and/or PC, and all four found that they played a role in predicting the development of pain. ${ }^{26}$

\section{Disability}

Higher levels of FABs and PC in patients have been found to be related to higher disability; ${ }^{8,28,94,97-103}$ for example, in a cross-sectional study of 96 participants with acute LBP, FABs were found to be strong predictors of a subject's ability to perform a lifting task. ${ }^{104}$ In fact, FABs and PC were better predictors of lifting capacity than Roland-Morris Disability

Questionnaire scores and pain intensity. ${ }^{104} \mathrm{~A}$ study of 211 participants with chronic pain found that patients with higher PC reported more disability and greater psychological distress. ${ }^{101}$ Grotle et al. examined FABs, distress, and disability in 356 participants with acute or chronic LBP. Patients with chronic LBP had more FABs and those beliefs were associated with greater disability and more work absenteeism. ${ }^{93}$ These findings suggest an association between patient beliefs and disability. The cross-sectional nature of the studies limits the ability to make inferences about a cause and effect relationship. It is possible that higher levels of disability result in elevated FABs and PC. Another limitation of these studies is the over reliance on selfreport measures of disability. FABs and PC may influence patients' self-reports of disability, 
irrespective of the patients' actual functional abilities. To avoid problems with self-reporting bias, future studies should include direct measurements of function, such as the lifting task utilized by Swinkels-Meewisse et al. ${ }^{104}$ The association between disability, FABs, and PC is a fairly consistent finding in the literature; however, the relationship is generally weak to moderate at best ${ }^{8,28,94,97-103}$ Given the complex nature of LBP, it is not surprising that patient beliefs may only explain a portion of the variability in disability. Despite only having a modest correlation, patient beliefs may be an important factor given that several studies have found FABs and PC to be the strongest single predictors of disability. ${ }^{28,93,98,104}$

\section{Pain Intensity}

$F A B$ and $P C$ have been shown to influence pain intensity in patients with

musculoskeletal pain. ${ }^{8,94,101,102,105,106}$ In 1998, Sullivan et al. studied 86 participants with persistent pain disorders (75\% with LBP). PC in these participants was moderately correlated with several factors, including present pain intensity (0.46), disability (0.55), depression (0.47), and anxiety (0.51).${ }^{94}$ This study suggests that there is a relationship between pain and PCS; however, due to study limitations it is unclear if this relationship is causal. A causal link between PC and pain intensity was supported in a prospective study published by Sullivan et al. ${ }^{107}$ In this study, PC was measured in 80 healthy college students without pain. Experimentally induced pain was measured using a visual analog scale and pain behaviors were recorded by independent raters who watched video recordings of the session. The results demonstrated PC was a predictor of pain intensity and pain behaviors for the female participants in the study. The prospective nature of the study suggests that PC may have an influence on pain intensity ratings and behaviors. This relationship was only seen in the female participants $(n=42)$ and it is 
unclear if the finding can be generalized to individuals with non-experimentally induced painful conditions. Peters et al investigated the relationship between pathology, FAB, PC, pain intensity, and disability in 100 patients with non-specific LBP. ${ }^{105}$ Disability had the strongest association with pain intensity; however, FAB and PC explained $4-10 \%$ of the variability in pain intensity. Overall, only $25 \%$ of the total variance in pain intensity was explained by the factors included in this study. This suggests that factors other than PC, FAB, pathology, and disability play a significant role in predicting pain intensity. Similar studies by Turner et al. ${ }^{102}$ and Severeijns et al. ${ }^{101}$ found a correlation between higher levels of PC and greater pain intensity in patients with chronic low back pain. Nearly all the studies investigating the relationship between patient beliefs and pain intensity in participants with painful conditions were retrospective, relied on self-reported measures, and did not include long-term longitudinal follow-ups. As a result, conclusions about cause and effect, and the long-term consequences of patient beliefs on pain intensity, cannot be made.

\section{Chronicity}

Patient beliefs about musculoskeletal pain may also have an impact on the development of chronic pain; ${ }^{24,28-30,98,108,109}$ however, the literature on this topic is limited by small sample sizes, prospective designs and is lacking in long-term longitudinal studies. The proposed hypothesis is that certain patient beliefs increase the risk of developing CLBP. The exact mechanism for this connection is unclear, but it is possible that patient beliefs influence neurophysiology or behavior in a way that increases the risk of developing long-term LBP symptoms. Several published studies have demonstrated a relationship between patient beliefs and CLBP. For example, Fritz et al. followed 78 participants for 4 weeks to determine factors 
that predicted continued symptoms. ${ }^{98,109}$ After 4-weeks, $29 \%$ of the participants remained on work restrictions. FABs were the best predictors of work status and disability at the 4-week follow-up. ${ }^{98,109}$ Klenerman et al. followed a sample of 300 patients with acute LBP for 1 year. ${ }^{28}$ Measurements were taken at one week and two months to identify factors that may predict outcomes at 12 months. Only $41 \%$ of the participants were measured at all three time points and one-year follow-up was obtained for $54 \%$ of the sample. FABs were the strongest predictor of persistent LBP over the 12 -month period, explaining $25 \%$ of the variability. ${ }^{28}$ Picavet et al. also performed a longitudinal study of patients with LBP in hopes of identifying predictors of CLBP. ${ }^{30}$ The follow-up time was half that of the Klenerman study; however, it involved a significantly larger sample size. Eighty five percent of the 1,845 surveyed participants $(1,571)$ responded to the survey and $26.2 \%$ reported $L B P$. FABs and PC predicted continued LBP, LBP severity, and disability with odds ratios ranging from 1.7 to 3.0. ${ }^{30}$ These studies investigating the influence of patient beliefs on the development of chronic LBP suggest that beliefs may be a risk factor for chronicity. These studies are limited by short-term follow-ups, patient drop-out, missing data, and a reliance on self-report.

\section{Treatment Success and Prognosis}

The impact of patient beliefs on prognosis and treatment success has been investigated by several authors. $31,59,95,96,98,100,110-114$ The majority of these studies have shown that higher levels of FABs and greater PC correlate with a worse prognosis and reduced effectiveness of traditional interventions utilized by physical therapists. A 2006 systematic review on FABs and LBP prognosis included 9 articles, 8 that were classified as having acceptable quality. ${ }^{112}$ This review included prospective studies that investigated the impact of FABs on the prognosis of 
patients with acute and subacute LBP. Three studies from this review demonstrated no association between FABs and prognosis. The other studies suggested a relationship, but the effect sizes were generally small. In 2007, Mallen et al. performed a broader systematic review that investigated prognostic factors for patients with musculoskeletal pain. ${ }^{95}$ They identified 11 prognostic factors including higher baseline pain intensity, multiple pain sites, lower social support, and greater movement restriction. Two of the identified factors, higher somatic perceptions/distress, and coping strategies, overlap with the FAB and PC constructs. In 2014, Wertli et al. published two systematic reviews investigating the influence of patient beliefs on prognosis. ${ }^{31,32}$ One of these reviews investigated the influence of FABs on patients with nonspecific LBP. ${ }^{65}$ The review included 21 studies and concluded that high FABs were prognostic of poorer outcomes for patients with sub-acute pain. The strongest connection was the association between FABs and the risk of work-related disability. ${ }^{31}$ The review did not find FABs to be prognostic for patients with LBP less than 2-weeks or greater than 3-months. The other review by Wertli et al. investigated the influence of PC on the prognosis of patients with LBP. ${ }^{66}$ The authors included 16 studies and found that most of the studies reported that PC was predictive of pain and disability in patients with acute, sub-acute, and chronic LBP. Both the reviews by Wertli and colleagues were limited by inconsistencies in the included studies, short follow-ups, and a reliance on self-reported measures. Due to the variability in study design, neither review included a meta-analysis of the data.

Similar to overall prognosis, patient beliefs may have an impact on the response a patient has to treatment..$^{59,96,113,115,116}$ For example, a patient who has fear about movement may be less likely to respond positively to an exercise based intervention. Bergbom studied 297 
patients receiving physical therapy and found a relationship between higher levels of PC and a lack of improvement with interventions. ${ }^{115}$ Al-Obaidi et al. also found that FABs were predictive of patients who did not respond to an exercise-based intervention. ${ }^{96}$ In contrast, Underwood et al performed a secondary analysis of 1334 participants who participated in the UK BEAM trial. They found that FABs were predictive of overall outcomes, but they were unable to specifically predict response to treatments such as exercise and spinal manipulation. ${ }^{113}$ In 2014, Wertli et al. performed systematic reviews to determine if FABs and PC were moderators or mediators of treatment efficacy. ${ }^{59,116}$ The reviews included 17 studies on FABs and 11 studies on PC, with study quality ranging from moderate to high. The reviews concluded that a reduction in FABs was a mediator of reduced disability in patients with LBP for less than 6months ${ }^{59}$ and a reduction of PC mediated improvements in pain and disability. ${ }^{116}$ The moderating effect of FABs and PC on treatment success were less consistent among the studies included in the reviews, which may have been due to a lack of power. ${ }^{59,116}$ These results suggest that changes in patient beliefs may be one of the factors that influence a patient's response to treatment.

\section{Interventions for FABs and PC}

Given the apparent influence of FABs and PC on LBP, there has been interest in interventions that target those beliefs. The current literature on this topic is limited in both quantity and quality; however, the available literature suggests that FABs and PC can be changed and those changes are associated with meaningful clinical improvements for patients with LBP. ${ }^{42,87,117-119}$ Two commonly utilized interventions that are aimed at addressing patient beliefs are Cognitive Behavioral Therapy (CBT) and Pain Neuroscience Education (PNE). CBT 
includes a large class of psychological treatments that attempt to address cognitive and behavioral aspects of pain, such as catastrophizing and avoidance behaviors. ${ }^{7}$ PNE is an educational intervention that teaches patients about the neurologic origins of pain, emphasizes the disconnect between tissue damage and pain, and seeks to reassure patients that pain reduction is possible. ${ }^{119,120}$ Studies on the effectiveness of CBT have generally found that it is a useful intervention for patients with LBP. ${ }^{87,121}$ A 2015 meta-analysis on the use of CBT for the treatment of non-specific LBP found CBT resulted in greater improvement in disability and pain when compared to a wait-list and European guideline-based active treatments that included exercise. ${ }^{122}$ Although significant, the positive effects of CBT on LBP were consistently small to moderate at best. ${ }^{122}$ A 2018 systematic review investigating CBT for the treatment of subacute LBP found that 5 of the 6 included studies favored CBT ${ }^{87}$ The included studies used different CBT protocols and diverse outcome measures, making it difficult to compare the findings. Comparison groups were also variable and included individual exercise, group exercise, primary care management, and no treatment. Similar to other studies on CBT, the effects of the intervention were modest in patients with subacute LBP. ${ }^{87}$

Similar to CBT, several studies have shown that PNE has a positive influence on patients with LBP. Moseley et al. compared PNE combined with physical therapy (PT) to care provided by general medical practitioners and found significant improvements in pain and disability at the one-year mark. ${ }^{123} \mathrm{~A}$ more recent multicenter randomized controlled trial found that PNE plus exercise was more effective than biomedical focused education and exercise. ${ }^{124}$ There were small to medium effects for pain, symptoms of central sensitization, disability, pain beliefs, and function in patients with CLBP. Several other studies have been performed on PNE 
and it has been shown to be associated with reduced pain, ${ }^{125,126}$ improved function, ${ }^{126,127}$ decreased $\mathrm{FABs}$ and $\mathrm{PC},{ }^{127-130}$ reduced anxiety and/or depression, ${ }^{127}$ improved physical performance, ${ }^{128,131}$ and reduced healthcare utilization. ${ }^{130,132}$ The influence of PNE on CLBP was also supported by a 2018 meta-analysis that included 7 randomized controlled trials. ${ }^{133}$ The analysis found moderate evidence for a small to moderate short-term improvement in pain with PNE and low level evidence for a small to moderate short-term improvement in disability. There was also low-level evidence for small to moderate effect on pain and disability at 3months. Unlike these studies on CLBP, Traeger et al. found that PNE did not have a positive effect on patients with acute LBP. ${ }^{134}$ They performed a randomized placebo-controlled trial on the use of PNE in 202 patients with acute LBP. Patients were randomized to receive PNE or placebo education, which included active listening but no material or advice. PNE was no better than the placebo education at reducing pain intensity at 3,6, or 12 months. The PNE group did have a small reduction in disability (7\%) at 1 week and 3 months, but this reduction may not be clinically meaningful, and the difference was not present at the 6- or 12-month follow-up.

\section{Clinician Beliefs}

Based on the growing body of literature demonstrating the importance of patientoriented psychological factors, including beliefs, it has become commonly accepted that clinicians should consider cognitive and psychological influences when treating patients with LBP. ${ }^{64,71}$ It has also been proposed that cognitive and psychological factors within clinicians may have an influence on the recovery of patients with LBP. ${ }^{55,135}$ This evidence is centered on the attitudes and beliefs of clinicians about the relationship between LBP, tissue damage, and 
functional expectations. ${ }^{10,136}$ Proponents suggest that clinicians who draw a stronger relationship between pain and tissue damage, and who have lower functional expectations for patients with LBP, have a negative impact on the recovery of their patients. ${ }^{10,11,46,137}$ There are several potential mechanisms for this negative effect and the evidence on this topic is still evolving.

\section{Impact on Clinician Behaviors}

The most direct potential influence of clinician attitudes and beliefs on the recovery of patients with LBP is the effect these attitudes and beliefs may have on the actions of the clinician. Twenty-three studies were identified that investigated the relationship between clinician attitudes and beliefs about LBP and their clinical actions. ${ }^{43,45-48,54,63,138-153}$ These studies included general practitioners, orthopedic surgeons, rheumatologists, chiropractors, manual handling advisors, physical therapists, and physical therapist students across 10 different countries (see Table 1). Twenty of the studies found a relationship between clinician attitudes and beliefs and clinical actions. All 20 of these studies found that clinicians who have lower functional expectations and/or stronger BPA-oriented beliefs were prone to clinical actions that encouraged less activity. ${ }^{43,45-48,54,63,138-140,142,144-150,152,153}$ For example, Coudeyre et al. measured the FABs of 864 general practitioners (GPs) in France and surveyed them about their usual actions when treating patients with LBP. ${ }^{142}$ The beliefs of GPs were measured using the Fear Avoidance Beliefs Questionnaire for Healthcare Workers (FABQ-HC) ${ }^{62}$ and clinical practice was measured using five questions, which inquired about the information GPs provided to patients with LBP, the referral practices of the GP, the length of sick leave prescribed for acute LBP, the advice given about physical activity, and the GP's attitudes regarding job adaptations, 
sick leave prescription, and physical activity for patients with chronic LBP. Approximately $16 \%$ of GPs were categorized as having high FABs (>14 on FABQ-HC Physical Activity subscale); GPs with higher FABs were more likely to suggest bed rest $(p<0.001)$, recommend longer work sickleave $(p<0.05)$, and were less likely to encourage patients to maintain a tolerable level of physical activity $(p<0.001)$. Lower FABs scores were associated with greater familiarity with functional restoration programs $(p=0.002)$, a larger focus on patient education $(p=0.004)$, referral to back schools $(p=0.01)$, and recommendations for less than 8 -days of sick leave $(p=0.003)$. Bishop et al conducted a similar survey-based study of 455 GPs and 580 physical therapists in the United Kingdom. ${ }^{48}$ Attitudes and beliefs were measured using the Pain Attitudes and Beliefs Scale (PABS-PT) and clinical practice was determined by asking questions about a patient vignette. The PABS-PT contains two subscales and scores indicate whether a clinician has beliefs that are more biomedically- or biopsychosocially-oriented. The responses to the clinical practice questions were categorized as being strictly in-line, broadly in-line, or not in-line with guideline recommendations. Strictly in-line advice included return to work, perform usual activities, and avoid bed rest. Not in-line advice included stay out of work until pain disappears, limit physical activity until pain disappears, and rest in bed until pain disappears. Advice labeled as not in-line for work, activity, and bedrest was given by $28.1 \%, 6.6 \%$, and $0.9 \%$ of respondents respectively. Mean biomedical scores were higher $(28.3,30.6,33.5)$ and mean biopsychosocial scores were lower $(34.1,33.3,31.8)$ for clinicians whose work advice was labeled as divergent from guideline recommendations $(p<0.001)$. The association between PABS-PT scores and advice about activity and bedrest were not analyzed due to the low number of respondents who provided advice that was labeled not in-line. Clinicians who scored high in 
biomedical and low in biopsychosocial on the PABS-PT made up the greatest percentage of individuals who provided advice not in-line with guidelines for physical activity and bedrest.

Houben et al. performed a study looking at attitudes and beliefs about low back pain, harmfulness perceptions about physical activity, and recommendations for activity of 295 therapists (manual therapists $n=113$, physiotherapists $n=69$, McKenzie therapists $n=57$, Chiropractors $n=26$, and others $n=30$ ) in the Netherlands. ${ }^{46}$ Attitudes and beliefs were measured using the PABS-PT and harmfulness judgments about physical activity were determined using the Photograph series of Daily Activities (PHODA), which consisted of 41 pictures of individuals performing common tasks. Study participants ranked the harmfulness of the PHODA tasks on a 7-point scale, ranging from "not harmful at all" to "extremely harmful." Recommendations for physical activity were determined using three patient vignettes. Study participants were asked to rate the level of pain, amount of pathology, and the recommended level of activity and work for each patient vignette. Scores on both PABS-PT subscales were predictors of PHODA harmfulness ratings $(p<0.01)$ and recommendations for physical activity $(p<0.01)$ in the expected direction. The biopsychosocial subscale was also predictive of work recommendations $(p<0.05)$.

There were three studies that failed to find a connection between attitudes and beliefs and the clinical actions of healthcare providers. Epstein et al. examined the FABs of 149 Israeli primary care providers and surveyed them about their knowledge and readiness to implement LBP guidelines. ${ }^{143}$ There was no association between FABs and the measured guideline variables, which suggests no connection between clinical actions and attitudes and beliefs. This study was limited by the high overall guideline knowledge of the sample, which may not have 
been representative of the population. The lack of variability in guideline knowledge may have also increased the chance of a type II error due to the small number of participants with poor guideline knowledge. The questions about clinical behaviors used in this study were oriented around guideline knowledge and did not ask about actual behavior. As a result, the findings of this study suggest no connection between guideline knowledge and attitudes and beliefs, but it is unclear if clinical behaviors were correlated. Another study which did not find a connection between attitudes and beliefs and clinical action was performed on 47 student physical therapists and recent graduates in Ireland. ${ }^{141}$ There was no connection between activity and work advice given for three patient vignettes and the biomedical and/or biopsychosocial orientation of the participants. The final study that failed to find a relationship between actions and beliefs determined that beliefs were not correlated with the number of sickness certifications issued by GPs in the United Kingdom. ${ }^{151}$ This was the only study of the 20 that directly measured clinical behaviors and did not rely on self-report or hypothetical patients.

In general, the literature suggests that the attitudes and beliefs of clinicians have an impact on the type of clinical actions they take. Those clinicians with higher FABs, lower functional expectations, greater biomedically-oriented beliefs, and less biopsychosocial orientation are more likely to suggest that patients limit their activity. This indicates that clinician attitudes and beliefs may play a role in the outcomes of patients with LBP. Despite this consistency, several limitations in these studies should be considered when generalizing the results to physical therapists. A major limitation is that only eight of the studies were performed exclusively on physical therapists, and of those eight, only one included physical therapists from the US. As a result, it is unclear if the bulk of the studies on this topic can be 
generalized to physical therapists in the US. Another significant limitation is the reliance on selfreported measures. Only one of the 23 studies included a direct measurement of clinician behavior and that study did not find a connection between beliefs and behaviours. ${ }^{151} \mathrm{It}$ is unclear if the self-reported measures of clinician behavior were reliable and valid. Even if clinician attitudes and beliefs had an impact on the behaviors of clinicians, it is unclear if these effects resulted in a significant influence on patient outcomes.

\section{Impact on Patient Beliefs}

Clinician attitudes and beliefs about LBP could impact patient outcomes more indirectly by influencing the beliefs of patients. ${ }^{55}$ As discussed previously, there is evidence that patient beliefs influence their experience of LBP and seem to have an impact on outcomes, such as pain intensity and disability. ${ }^{32,95}$ If clinician attitudes and beliefs have an effect on the beliefs of patients, it is possible that this influence will have an impact on patient recovery. Six studies were identified that investigated the impact of clinician attitudes and beliefs on the beliefs of patients. ${ }^{47,56,144,154-156}$ All six of these studies suggest that patient beliefs were influenced by the beliefs of the clinicians who cared for them (see Table 2). A good example of this influence was provided by Darlow et al. in a qualitative study of 23 patients with LBP from New Zealand. ${ }^{56}$ These patients, who participated in semi-structured interviews, described several factors that influenced their beliefs about LBP, including the media, family and friends, previous experience and healthcare professionals. Participants reported that healthcare professionals had the largest impact on their beliefs about the source and meaning of pain, and on their expectations for recovery. Clinicians who stressed protective avoidance strategies for the back and who focused primarily on BPA explanations for LBP, such as faulty alignment, ligament sprains, 
muscle dysfunctions, and postural factors, caused patients to feel guilt, frustration, and worry. For example, in response to receiving a diagnosis of a lumbar strain, one participant stated, "when I get that sharp pain, I guess that I've moved in a way that's continually putting strain on an area of the muscle that I've damaged....my assumption would be that I was making it worse." ${ }^{16}$ Patients also reported that BPA-oriented clinicians encouraged them to focus more on their back pain and promoted an attitude of protection. One patient who was told by a chiropractor that his back was out of alignment stated, "the only thing that was going through my mind is the seriousness of my dis-alignment...I was really petrified." Clinicians who focused on reassurance and activity promoted increased confidence, a positive attitude towards activity, and higher expectations for recovery. Poiraudeau et al. surveyed 286 rheumatologists and 443 of their patients to determine their beliefs about LBP. ${ }^{144}$ Physician beliefs were measured using the FABQ-HC and patient beliefs about LBP were measured using the Fear Avoidance Beliefs Questionnaire (FABQ). An association was found between patient FABs about physical activity and lower patient educational level, workplace physical demands, the patients' perceived level of disability, and physician FABs. High physician FABs were the strongest predictor of elevated patient fear of activity with an odds ratio of 5.92 (95\% confidence interval of 1.3-26.32). It is unclear from this data if physician beliefs caused the increased FABs in patients; however, based on the study by Darlow et al. it appears possible. ${ }^{56}$

A similar correlation between healthcare provider attitudes and beliefs and patient beliefs was found in a large study of Norwegian patients and clinicians. ${ }^{154}$ Four hundred and sixty nine healthcare workers (physical therapists $(n=255)$, physicians $(n=193)$, and chiropractors $(n=21))$ and 1502 patients were surveyed to determine their beliefs about LBP. 
Patients were recruited using phone calls to 2,717 residents of three counties in Norway.

Questionnaires were sent to all the practicing physicians $(n=414)$, physiotherapists $(n=663)$, and chiropractors $(n=28)$ in the counties. Both clinician and patient beliefs were identified using six statements that mirrored recommendations made by Norwegian guidelines for acute LBP or messages contained in a recent media campaign. The six statements were: 1) "if you have a slipped disc you should have surgery," 2) "radiograph and newer imaging tests can always find the cause of pain," 3) "bedrest is the mainstay of therapy," 4) "in most cases, back pain recovers by itself in a couple of weeks," 5) "back pain recovers best by itself," and 6) "one recovers faster from back pain if one continues at work or returns as soon as possible." Survey respondents rated their agreement with the statements on a 5-point scale, ranging from totally disagree to totally agree. Overall, clinician beliefs were in-line with the guideline recommendations and there were not significant differences between the various provider types. The exception was with statements four and five, which asked about the natural course of LBP. None of the chiropractors agreed with statement five and $71.4 \%$ disagreed with the statement. Only $4.8 \%$ of chiropractors agreed with statement four, $57.1 \%$ were unsure, and 38.1\% disagreed. These responses were significantly different than the responses given by physical therapists and physicians, who were more likely to agree with the statements $(p=0.001)$. Patients who reported being treated by chiropractors were also more likely to disagree with statements four and five than patients who were treated by physical therapists or physicians $(p=0.001)$. As with the study by Poiraudeau, ${ }^{144}$ it is unclear if this association indicates a causal relationship; however, it is plausible that the beliefs of chiropractors had an influence on the beliefs of their patients. It is also possible that BPA-focused chiropractors tend 
to attract patients who have similar attitudes and beliefs. Based on this limited data, it is possible that clinician attitudes and beliefs about LBP have an influence on the beliefs of patients. If patients develop higher FABs or PC due to interactions with healthcare providers, patient prognosis could be lower.

\section{Impact of Expectations}

There may be other, indirect ways that clinician beliefs influence the outcomes of patients with LBP. One of these may be the impact of clinicians' expectations about LBP and treatment efficacy. The influence of expectation on an outcome was first described by Rosenthal in the 1960s and has been labeled the observer-expectancy effect. ${ }^{65}$ Rosenthal observed that when his lab assistants believed that a rat was more intelligent, the rat was able to finish a maze faster than identical rats that were viewed as normal. This observation implies that the expectations of a tester, or perhaps a clinician, could influence the performance of a subject or patient. Galer et al. tested this phenomenon in a clinical setting by investigating the effectiveness of intravenous injections and nerve blocks for 46 patients with chronic pain. ${ }^{66}$ Patients treated by physicians who expected the intervention to work were more likely to have a positive outcome $(r=.42)$. A lack of clinical equipoise was also found to be a factor in outcomes after treatment with acupuncture. Witt et al examined the influence of 2781 physicians' expectations on pain reduction in 9900 patients after receiving acupuncture treatments. ${ }^{157}$ When added to the regression model, physician expectation had a small, but significant impact on outcomes. Participants treated by physicians who expected substantial improvement with accupuncture had less pain and better functioning after treatment. This effect was independent of participants' baseline characteristics, which suggests the clinician 
expectations were not based on observed patient prognostic factors. A study of student physical therapist in the Netherlands also suggests that clinician expectations may influence outcomes. ${ }^{9}$ In this study, participants who were tested on a lifting task by testers with higher FABs had significantly lower lifting capacity. If the lifters also had high FABs, the lifting capacity was reduced by $14.4 \mathrm{~kg}$ versus an $8 \mathrm{~kg}$ reduction if the lifter did not have elevated FABs. This suggests that clinicians with suboptimal beliefs may have the largest negative impact on patients who share those beliefs. ${ }^{9}$

The mechanism by which clinician expectations influence patient outcomes is not fully understood. It is possible that clinicians act differently based on their expectations and these actions may have a direct or indirect influence on patients. These changes in clinical behavior may be overt, such as changes in interventions, but the studies discussed above suggest that the influence may be more subtle. The clinicians' expectations may cause slight changes in verbal and non-verbal communication, which subconsciously influences the patient. ${ }^{158}$ Rosenthal proposed that the researchers' expectations about the intelligence of the rats caused the testers to handle the rats differently. ${ }^{65}$ This slight change in handling may have influenced the rats and caused them to be faster through the maze. It is reasonable to think that clinician expectations may influence their communication and handling of patients. These changes may influence outcomes by modifying the patient's confidence in the provider and the interventions. $^{158-160}$ 


\section{Impact on Outcomes}

The research previously discussed provides indirect support for the hypothesis that the outcomes of patients with LBP can be influenced by the attitudes and beliefs of clinicians. This effect may be mediated by an impact on clinician clinical behaviors, by changes in patient beliefs, and/or changes in clinician or patient expectations. Despite the indirect support for the hypothesis, direct evidence for a connection between clinician beliefs and patient outcomes is lacking. One challenge is that factors influencing outcomes are numerous and complex, ${ }^{161,162}$ which makes it difficult to identify individual factors, especially if they only have a small impact on outcomes. Four studies that investigated the impact of healthcare workers' attitudes and beliefs about LBP on patient outcomes were identified. ${ }^{144,148,163,164}$ Only one of the four studies concluded that clinician attitudes and beliefs had an impact the outcomes (see Table 3). This study by Beneciuk et al. put 6 physical therapists through an education session emphasizing a stratified treatment approach (including 8 hours on psychologically informed treatment) and 6 physical therapists through standard education. ${ }^{164}$ The physical therapists were then asked to treat patients based on the education they received and outcomes were obtained for their patients after 4 weeks. Physical therapists in the stratified education group had changed their attitudes and beliefs and patients treated by those physical therapists had greater improvements in pain and disability; however, it is unclear if the improved outcomes were a result of changes in the clinician beliefs. The physical therapists were instructed to treat based on the stratified education and it is unknown if the physical therapists would have changed their clinical behaviors simply based on changes in their attitudes and beliefs. There was also minimal change in the physical therapists' attitudes and beliefs after the education and it is 
unclear if those small changes had an impact on clinical behaviors or outcomes. The authors did not directly analyze if changes in attitudes and beliefs correlated with outcomes. In contrast to the findings of this study, Overmeer et al. did not find a relationship between changes in physical therapists' attitudes and beliefs and patient outcomes after an educational intervention. ${ }^{163}$ In this study, 42 physical therapists were randomized to an education intervention focused on BPS aspects of pain or to no education. Unlike the previously discussed study, participants in this study were not specifically instructed to follow a particular treatment protocol when treating patients. The outcomes of the patients from physical therapists in both groups were collected at baseline and at 6 months and there were no statistically significant differences between the groups; furthermore, the impact of physical therapist attitudes and beliefs on outcomes was not affected by patient levels of PC or depression. The authors did note a trend towards better outcomes in patients with high PC and depression who were treated by physical therapists in the intervention group, but that trend was not significant. The two other identified studies also failed to show that healthcare provider attitudes and beliefs had an impact on outcomes. Poiraudeau et al. measured the outcomes of patients with LBP who were treated by 266 rheumatologists. ${ }^{144}$ Ten percent of physicians had high FABs and they were more likely to recommend bed rest and time away from work. Despite those changes in behaviors, there was no association between patient outcomes and physician FABs. In another study involving physicians, Sieben found no correlation between physician attitudes and beliefs and their self-reported treatment choices or patient outcomes. ${ }^{148}$ 


\section{Literature Gaps}

The current studies investigating the impact of the attitudes and beliefs of physical therapists on the outcomes of patients with LBP are conflicting and limited. Both identified studies assessed the impact of changes in attitudes and beliefs and did not consider the impact of attitudes and beliefs separate from an educational intervention. ${ }^{163,164}$ Although several studies have shown clinician attitudes and beliefs can be changed through education, ${ }^{165-168}$ it is likely that it would take more than just a short educational intervention to change the behavior of the physical therapists in a meaningful way. ${ }^{169}$ The educational interventions used in the studies may also have limited effectiveness and a different educational strategy may be better at changing attitudes, , and behaviors. The skills needed to treat patients based on the BPS model are complex, and it may take years after having a change in attitudes and beliefs for a physical therapist to become adept at implementing these skills. It is also unclear if the beliefs of the physical therapist who participated in the two studies were problematic. It is possible that physical therapists' attitudes and beliefs will not impact outcomes unless they significantly deviate from the norm. With the small sample sizes utilized in these studies, it is possible that there was limited variability in the attitudes and beliefs of the participants. Attitudes and beliefs in the Beneciuk et al. study did not vary significantly and only changed minimally after the intervention. ${ }^{164}$ The actual attitudes and beliefs scores for physical therapists participating the Overmeer et al. study were not reported, so it is unclear if the study included participants with suboptimal attitudes and beliefs. ${ }^{163}$ The authors noted in their discussion that many of the physical therapists participating in the study already had an interest in psychologically informed 
PT. This may suggest that there was minimal deviation in attitudes and beliefs among physical therapists in the study.

Based on these limitations, there is a need for studies that investigate the impact of physical therapist attitudes and beliefs on patient outcomes without a focus on interventions aimed at changing those beliefs. It is also important to have studies that include a larger sample of physical therapists and patients who have divergent attitudes and beliefs. The main purpose of this study was to determine if physical therapist attitudes and beliefs about LBP are associated with the outcomes of patients with LBP. Secondary purposes of this project will be to describe the attitudes, beliefs, and pain science knowledge of physical therapists, and to determine the factors that influence the attitudes and beliefs of physical therapists. Several studies have described the attitudes and beliefs of physical therapists about LBP; however, of the identified studies on the topic, only 3 included physical therapists from the US. ${ }^{10,145,164}$ Several studies have also attempted to determine factors which influence the development of clinician attitudes and beliefs. ${ }^{63,142,143,170-173}$ The results of these studies are mixed and no clear consensus on factors that influence the development of attitudes and beliefs about LBP has emerged. Factors such as gender, ${ }^{171}$ experience treating patients with LBP, ${ }^{171}$ profession, ${ }^{170}$ years of school, ${ }^{173}$ and personal history of $\mathrm{LBP}^{173}$ have been implicated as factors that may influence the development of attitudes and beliefs about LBP. Understanding the factors that influence the development of attitudes and beliefs about LBP will be useful when designing strategies to improve those beliefs. 


\section{Summary}

LBP is a common disorder that results in significant disability and financial burden

globally. ${ }^{73}$ It has been suggested that classic models of LBP, which focus solely on BPA factors, should be expanded to include psychological and social factors. ${ }^{78}$ Patient beliefs about LBP such as FABs and PC may increase the risk of developing LBP and may impact pain intensity, disability, and prognosis. The attitudes and beliefs of clinicians about LBP appear to impact clinical behaviors, such as activity level recommendations, and they may have an impact on the outcomes of patients with LBP. The current literature investigating the impact of physical therapists attitudes and beliefs on the outcomes of patients with LBP is limited. This project expands the current body of evidence by describing the LBP-related attitudes, beliefs and knowledge of physical therapists in the US, by identifying factors that influence the development of those beliefs, and by studying the impact of physical therapist attitudes and beliefs on the outcomes of patients with LBP. 


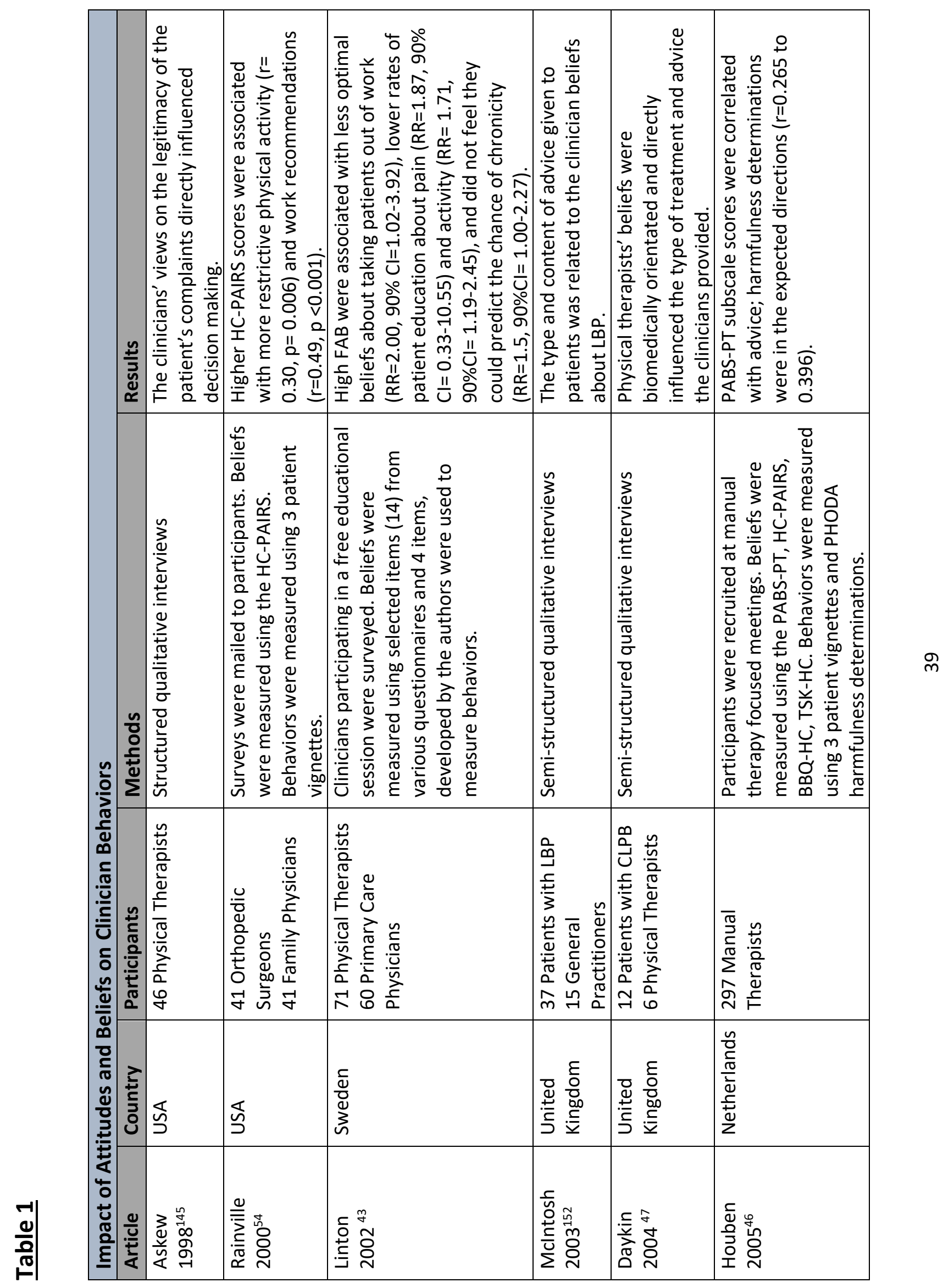




\begin{tabular}{|c|c|c|c|c|}
\hline 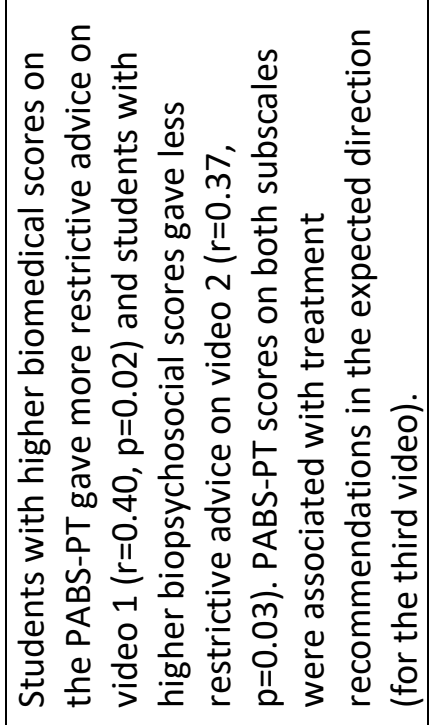 & 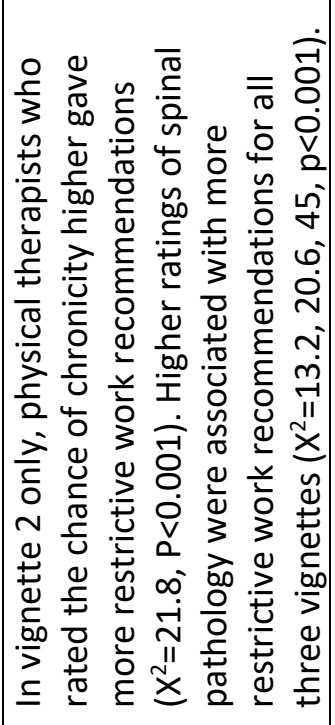 & 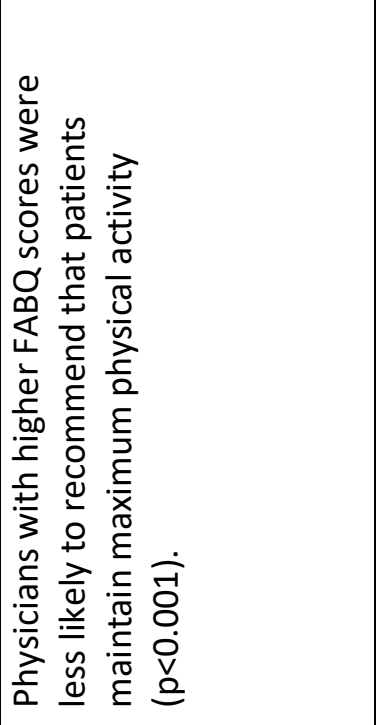 & 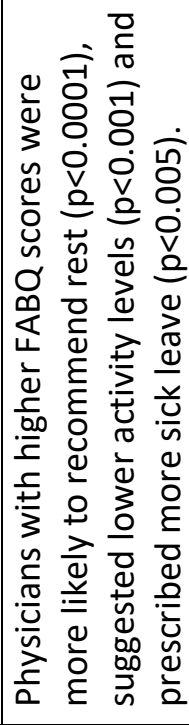 & 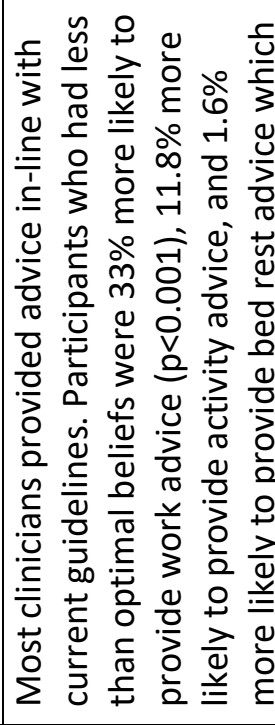 \\
\hline 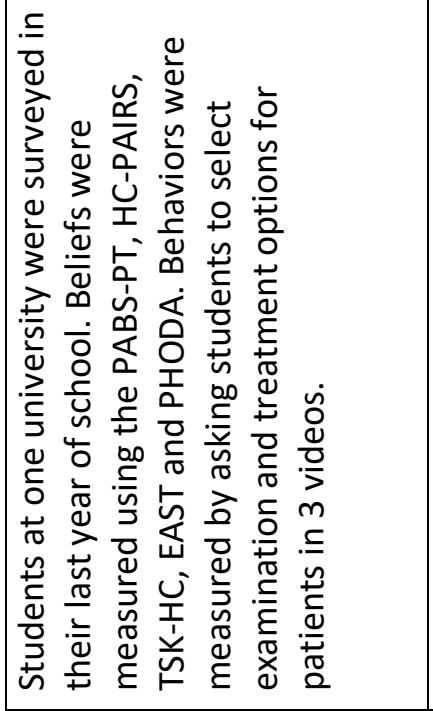 & 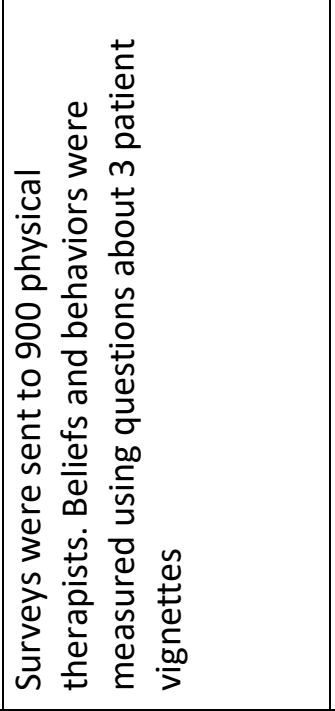 & 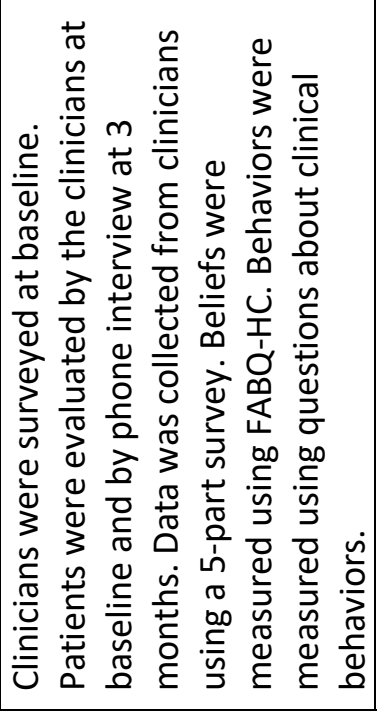 & 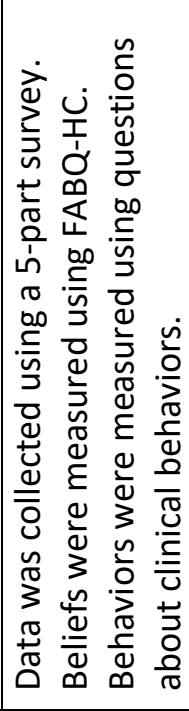 & 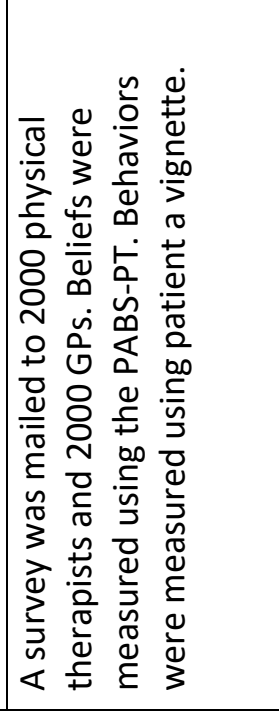 \\
\hline 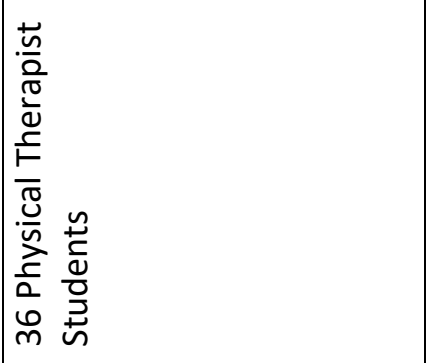 & 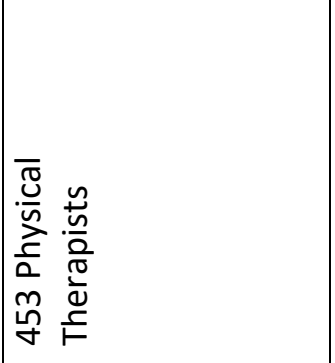 & 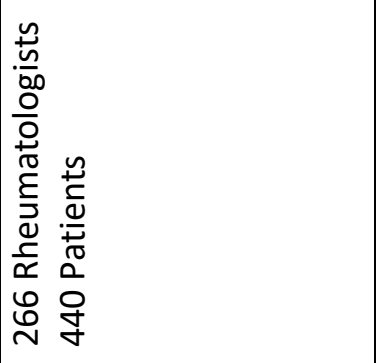 & 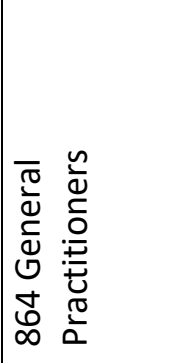 & 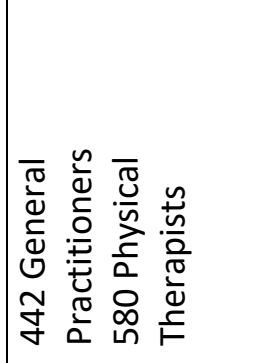 \\
\hline $\begin{array}{l}\frac{n}{0} \\
\frac{1}{0} \\
\frac{0}{1} \\
\frac{1}{1} \\
\frac{ \pm}{2} \\
z\end{array}$ & 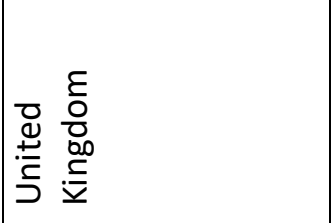 & & 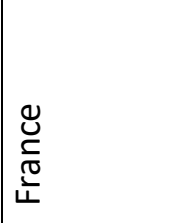 & 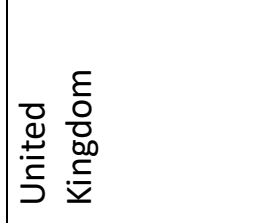 \\
\hline 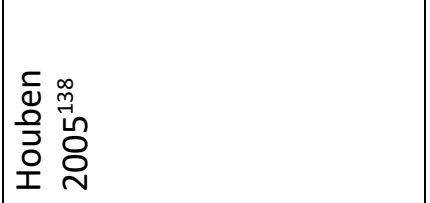 & 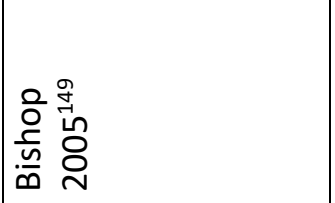 & 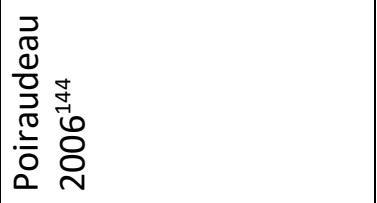 & 竞 & 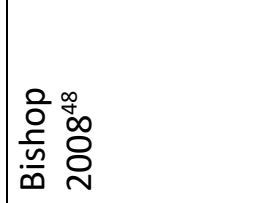 \\
\hline
\end{tabular}




\begin{tabular}{|c|c|c|c|c|c|}
\hline \multirow[t]{2}{*}{ 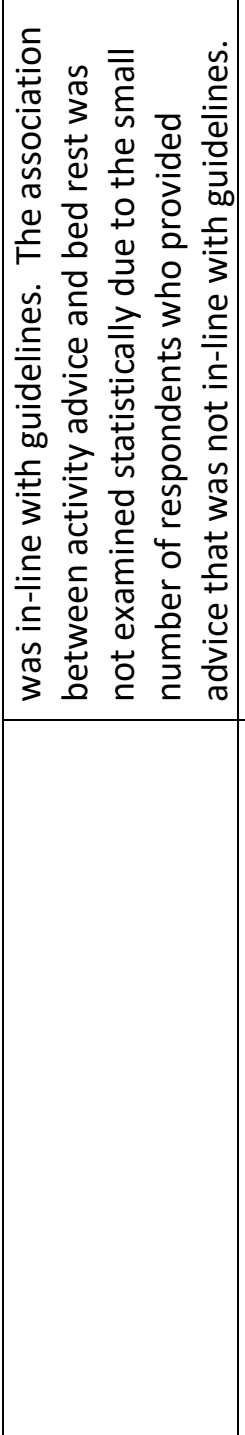 } & 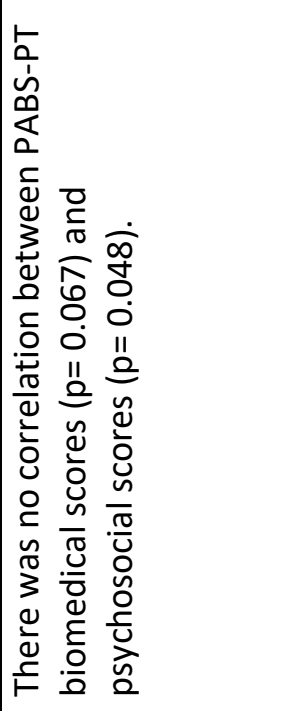 & 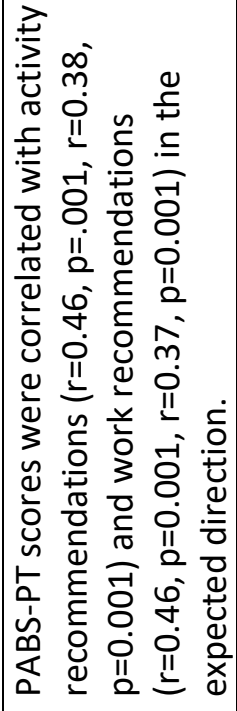 & 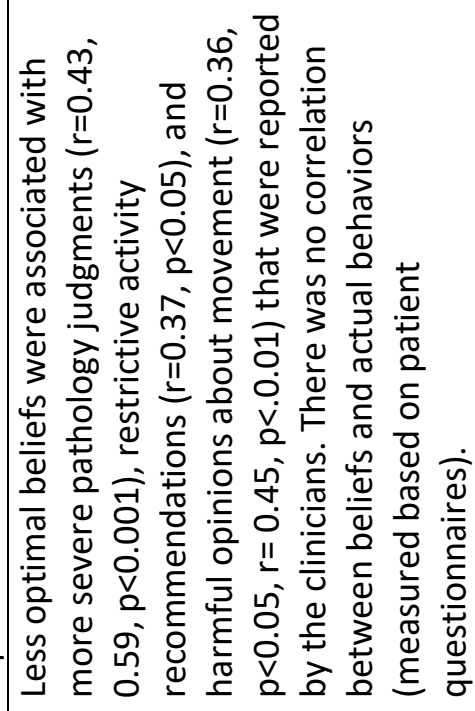 & 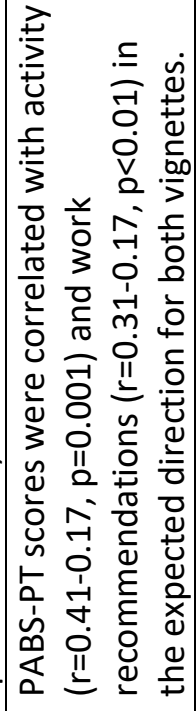 & 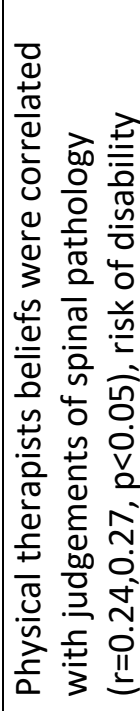 \\
\hline & 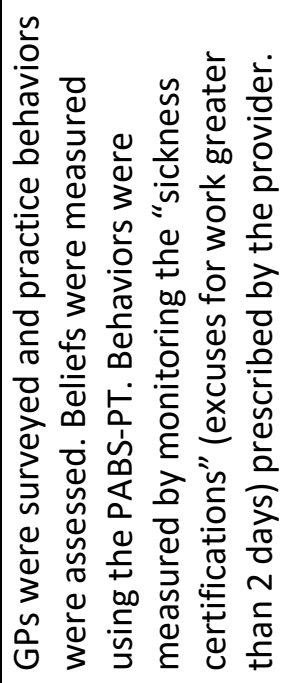 & 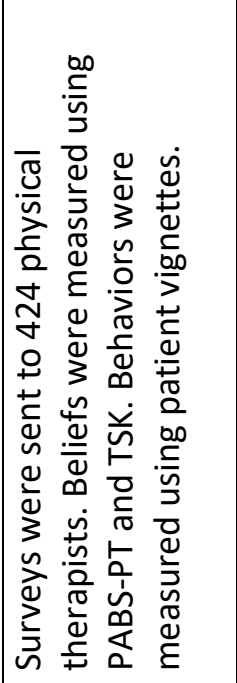 & 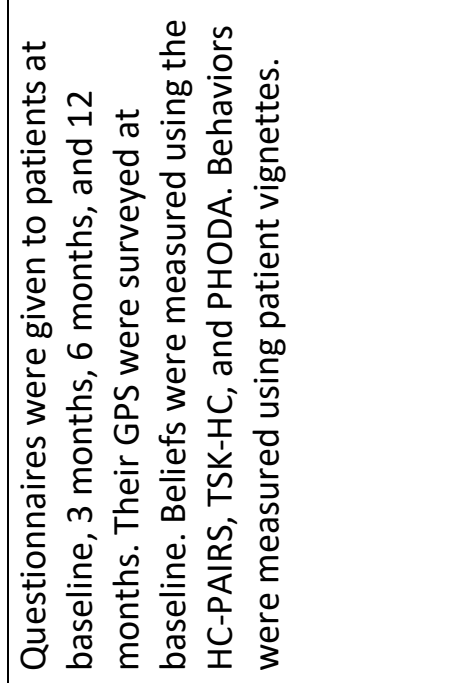 & 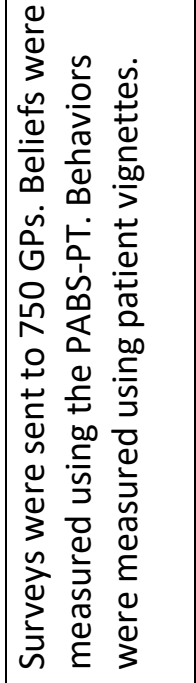 & 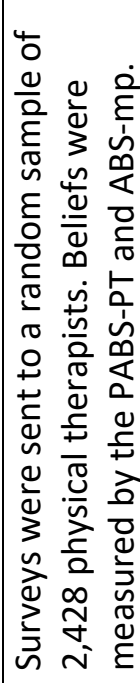 \\
\hline & 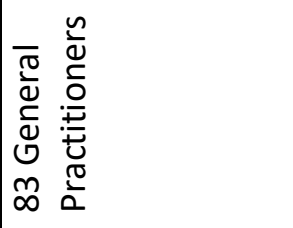 & 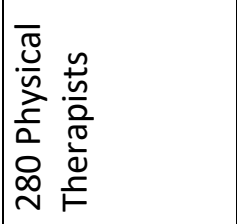 & 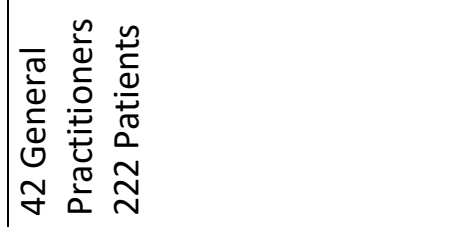 & 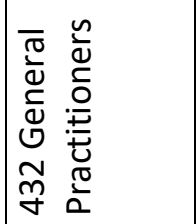 & 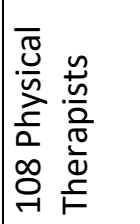 \\
\hline & $\begin{array}{l}\frac{D}{\frac{D}{0}} \\
\frac{\pi}{20} \\
\frac{\mathrm{U}}{w}\end{array}$ & 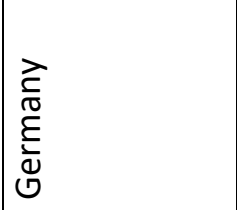 & $\mid \begin{array}{l}\frac{n}{0} \\
\frac{1}{0} \\
\frac{\pi}{2} \\
\frac{d}{d} \\
\frac{1}{0} \\
z \\
z\end{array}$ & $\begin{array}{l}\frac{0}{\frac{0}{0}} \\
\frac{\sigma}{0} \\
\underline{\underline{\underline{0}}}\end{array}$ & $\begin{array}{l}\frac{\pi}{0} \\
\frac{1}{\pi} \\
\frac{1}{10} \\
u\end{array}$ \\
\hline & 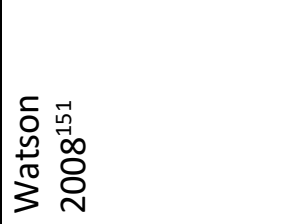 & 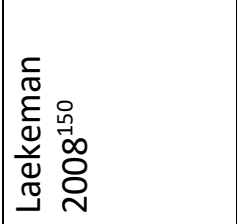 & 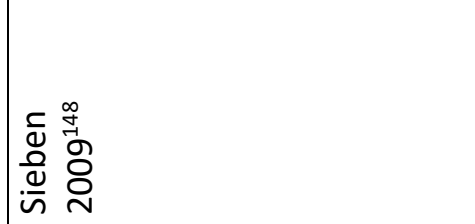 & 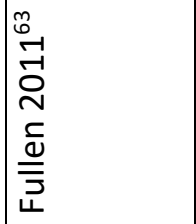 & 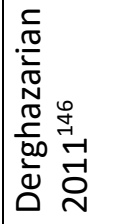 \\
\hline
\end{tabular}




\begin{tabular}{|c|c|c|c|c|c|c|}
\hline 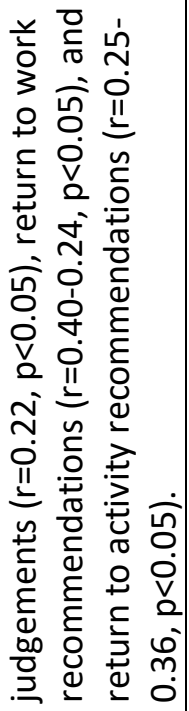 & 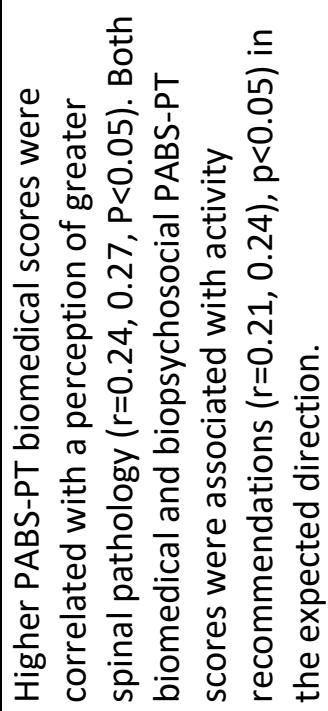 & 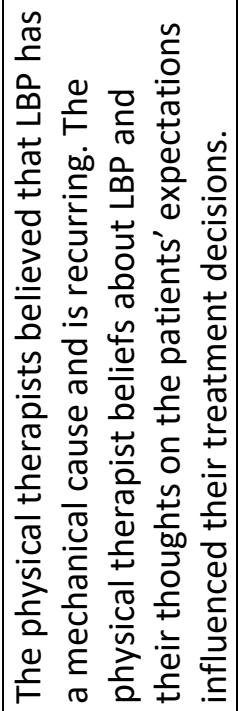 & 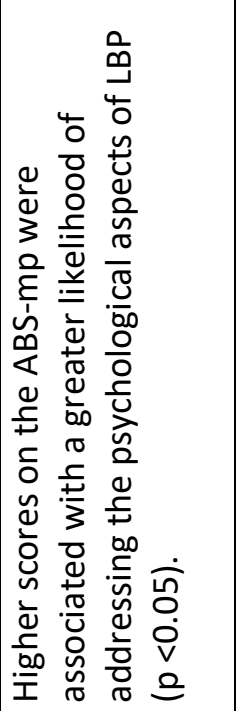 & 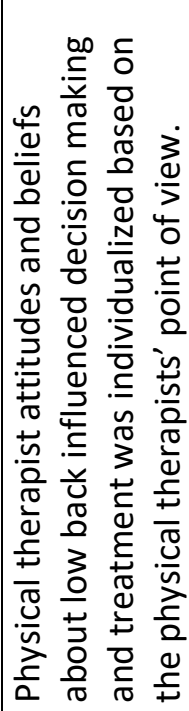 & 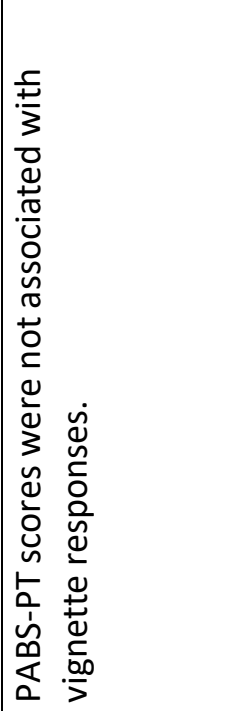 & 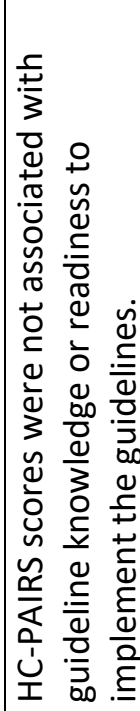 \\
\hline \multirow[t]{4}{*}{ 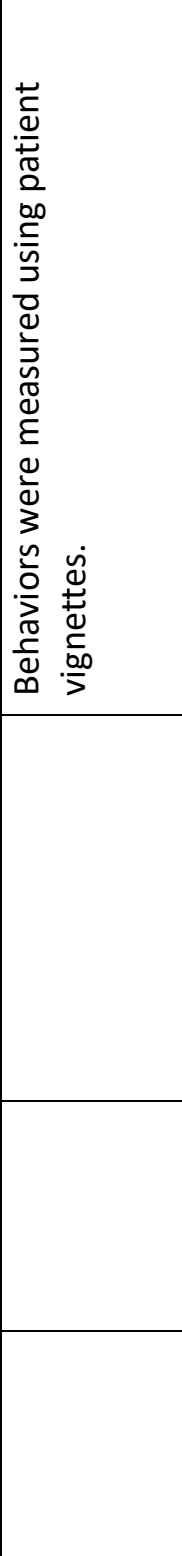 } & 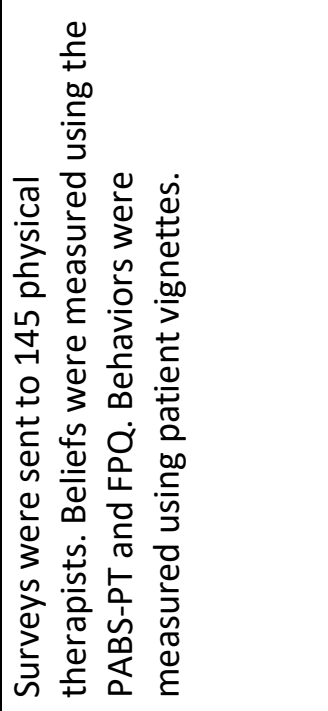 & 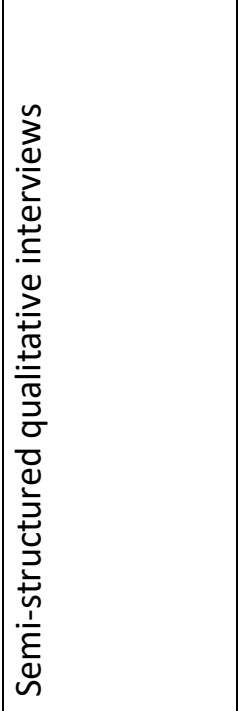 & 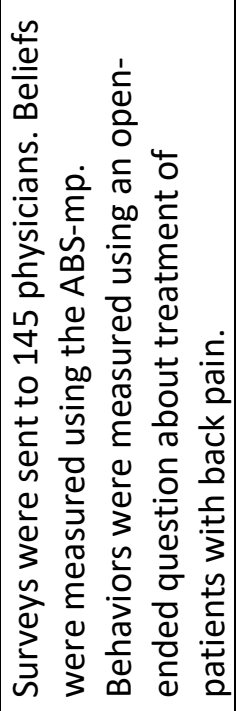 & 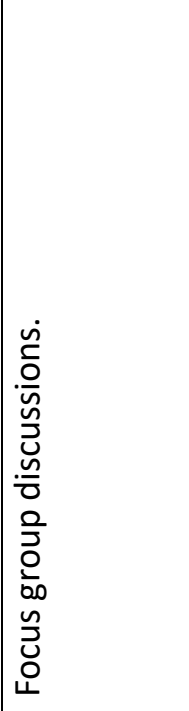 & 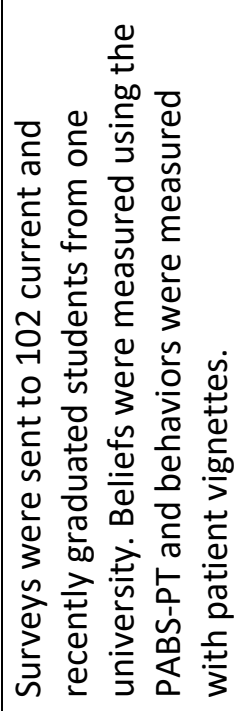 & 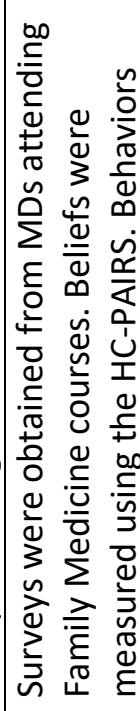 \\
\hline & 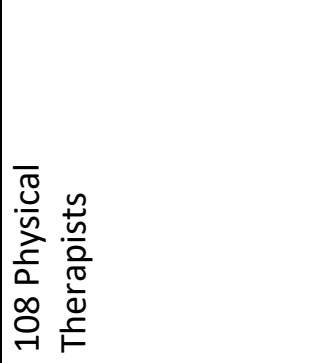 & 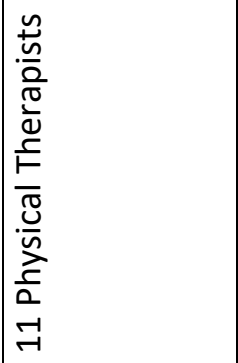 & 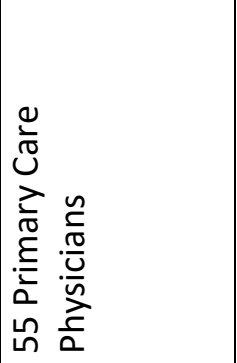 & 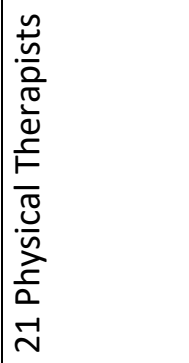 & 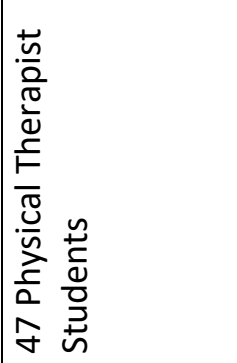 & 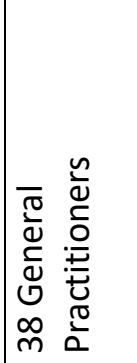 \\
\hline & $\begin{array}{l}\frac{\pi}{0} \\
\frac{\pi}{2} \\
\frac{1}{0}\end{array}$ & 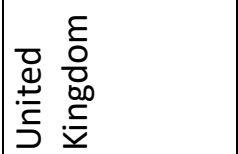 & $\frac{\frac{O}{\frac{0}{\pi}}}{\frac{\pi}{\frac{1}{L}}}$ & 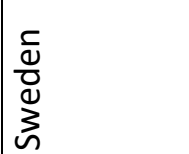 & 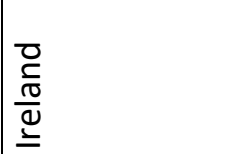 & $\begin{array}{l}\bar{\Phi} \\
\bar{v} \\
\underline{\omega}\end{array}$ \\
\hline & 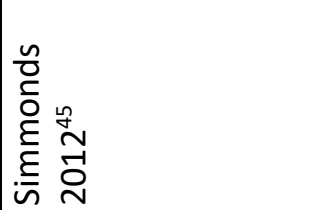 & 离亮 & 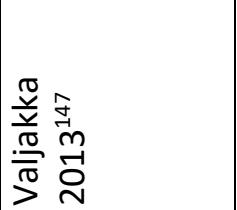 & 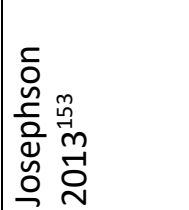 & 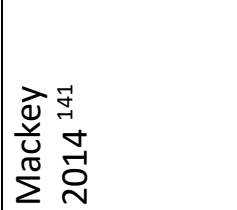 & 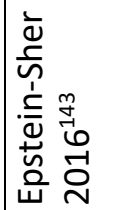 \\
\hline
\end{tabular}




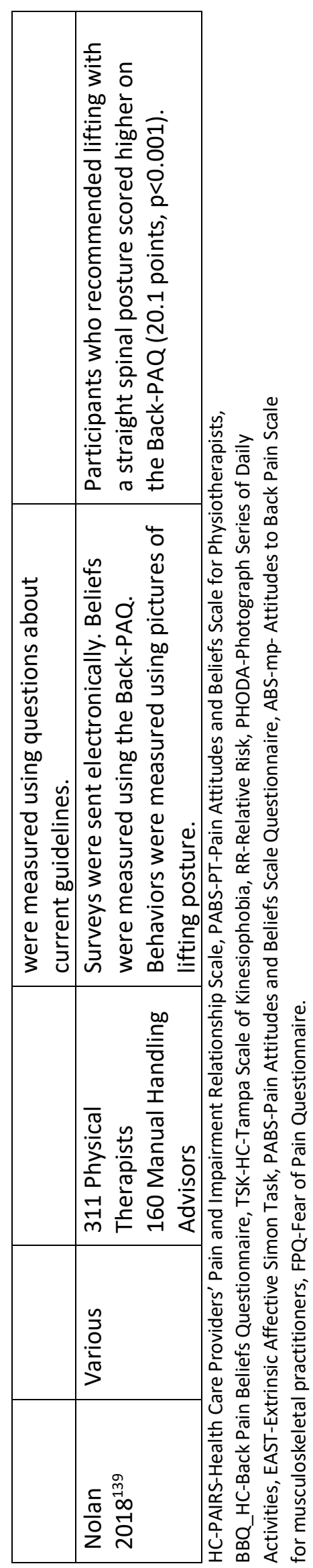

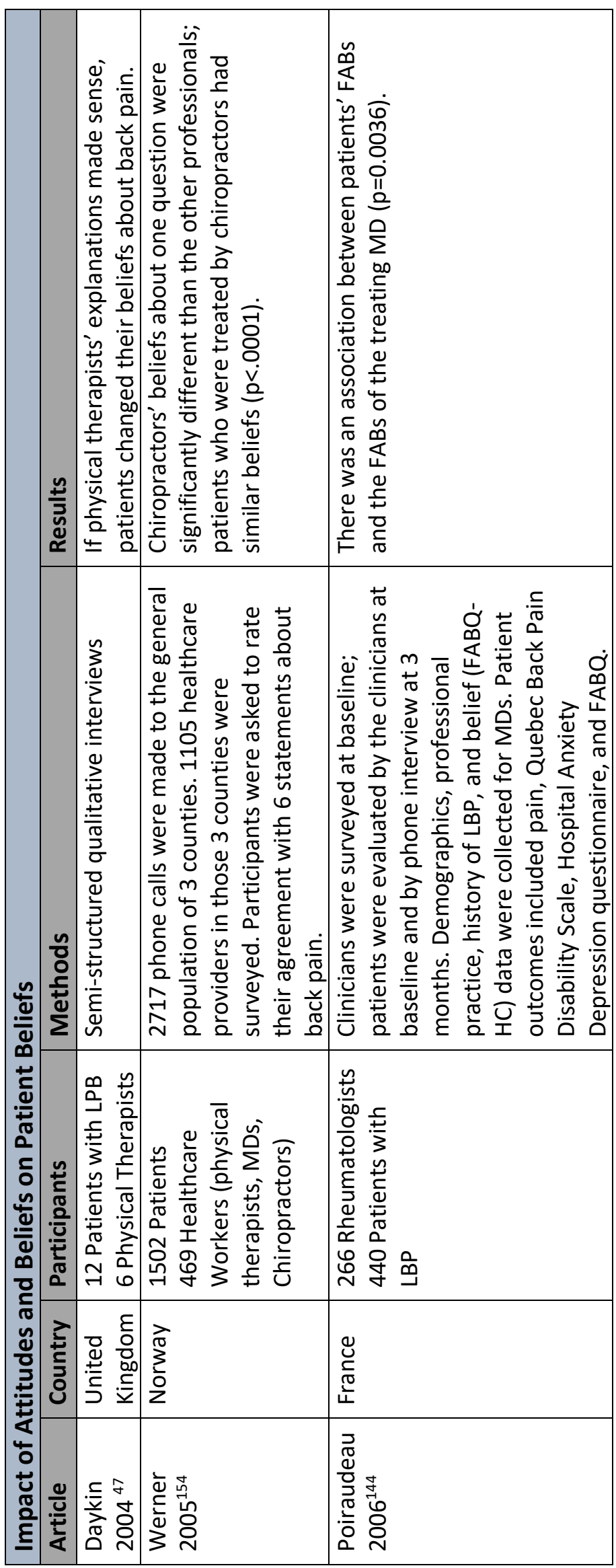




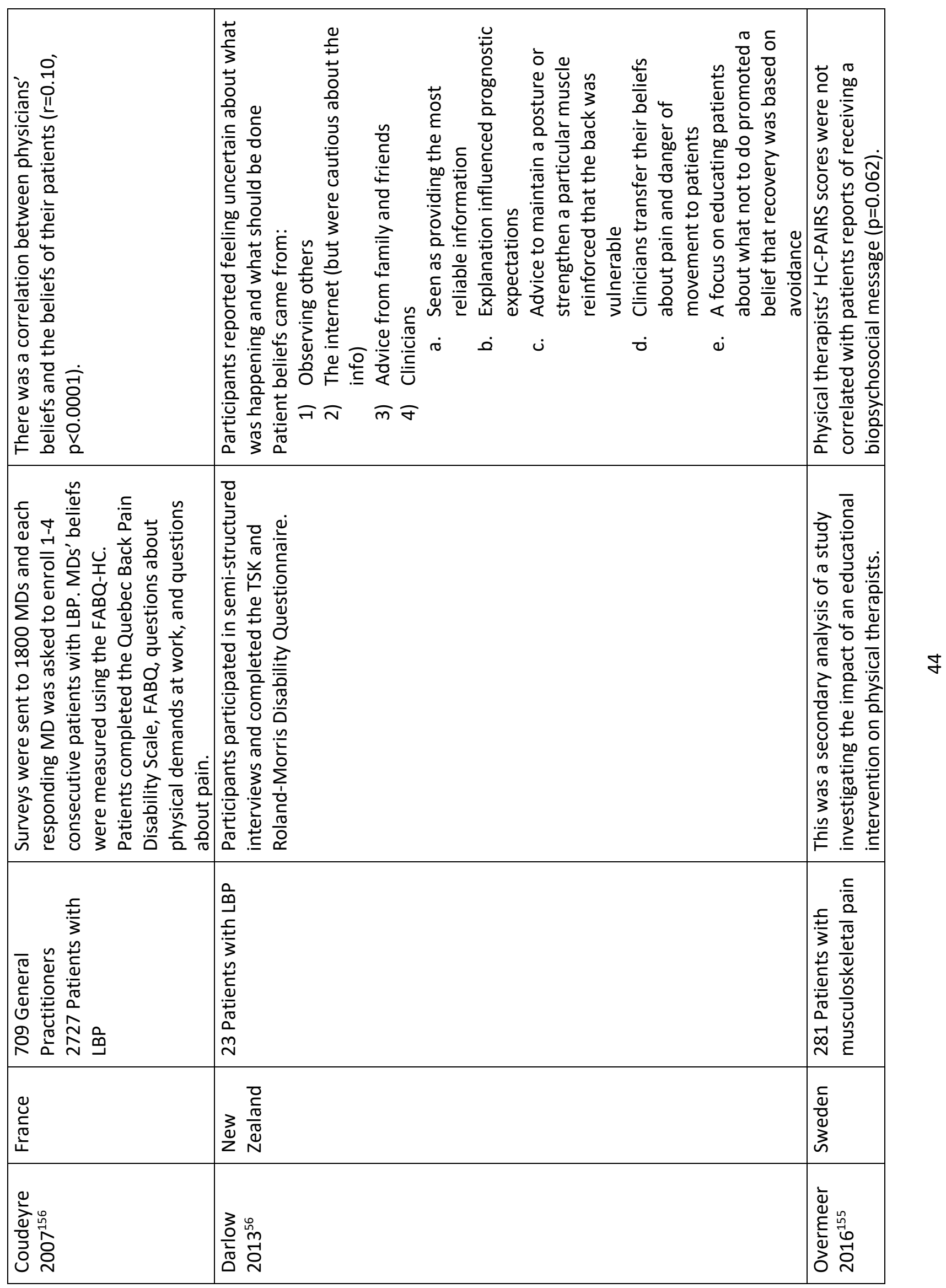



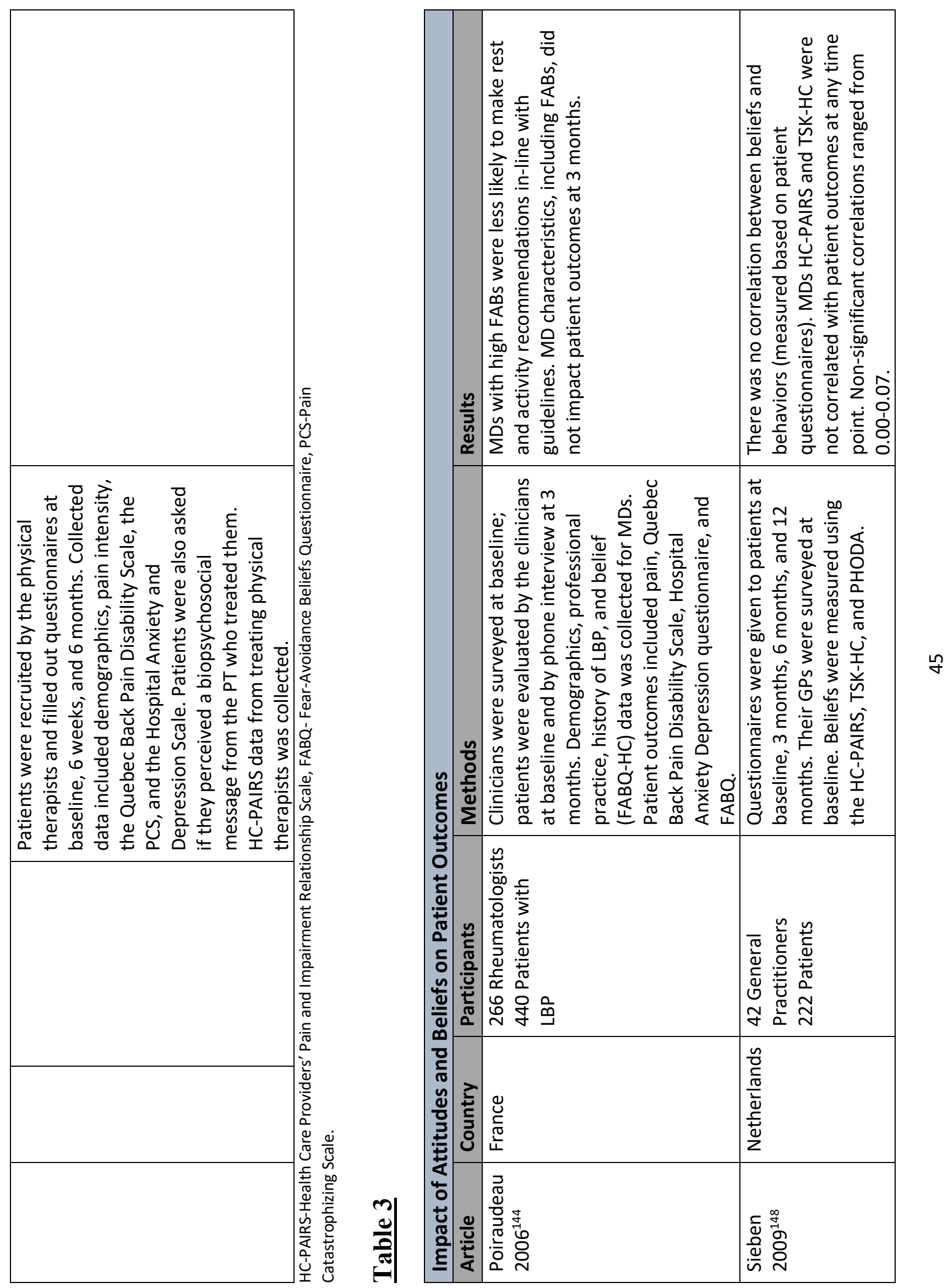


\begin{tabular}{|c|c|c|}
\hline & 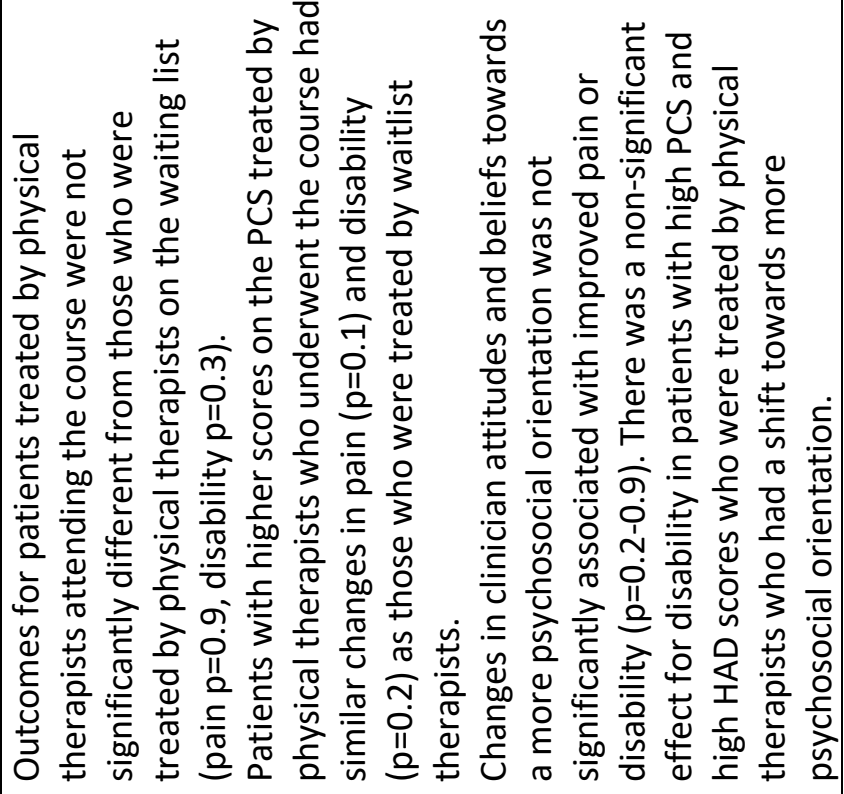 & 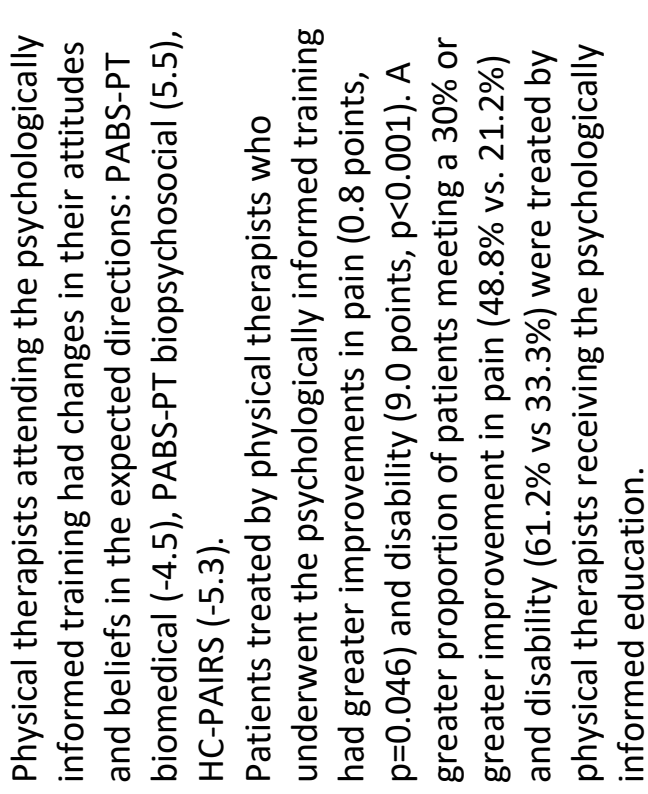 \\
\hline 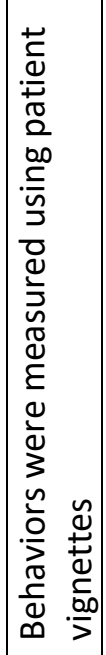 & 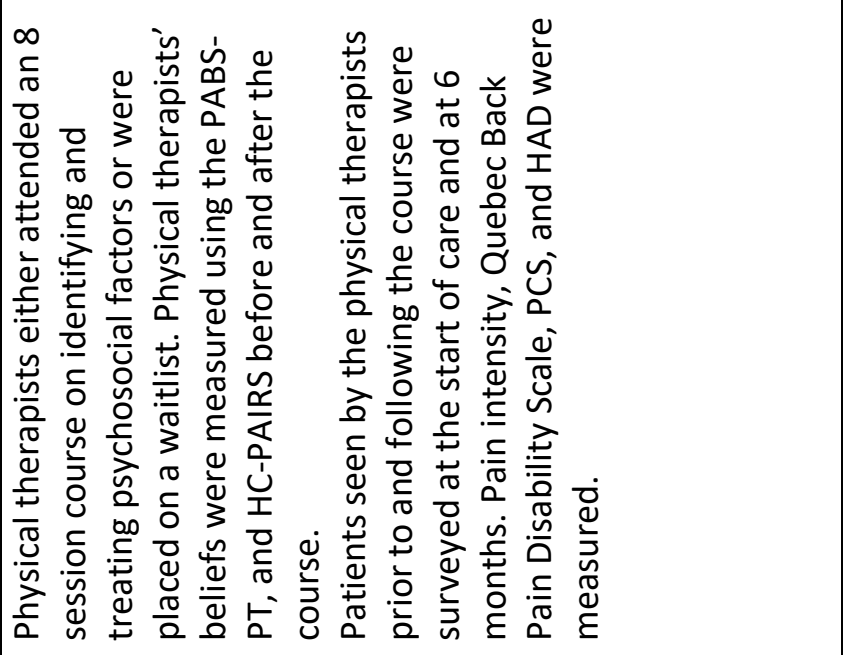 & 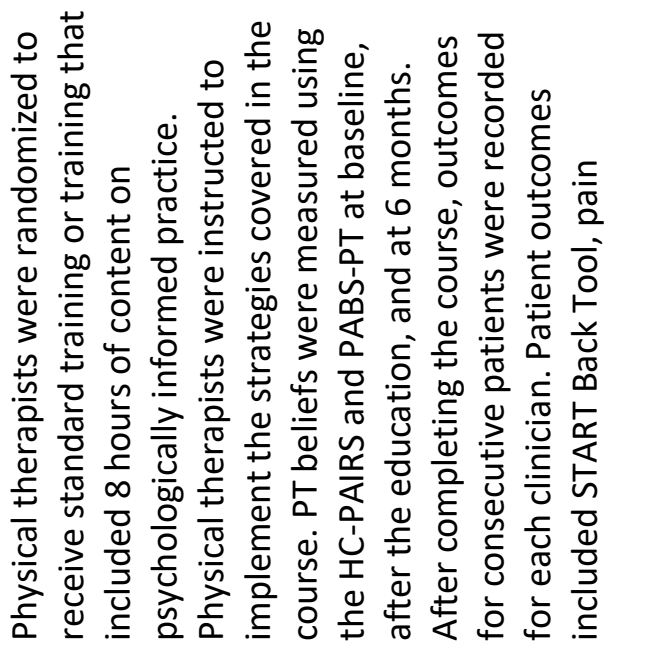 \\
\hline & 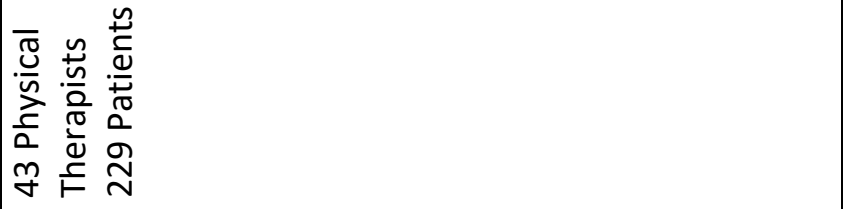 & 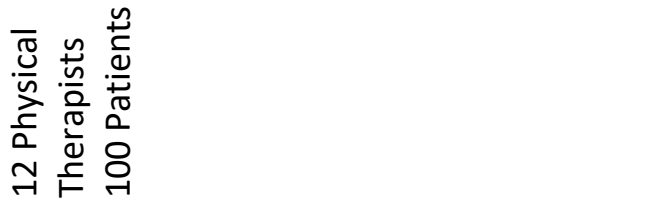 \\
\hline & $\begin{array}{l}\frac{c}{d} \\
\frac{d}{d} \\
\sum_{n}\end{array}$ & 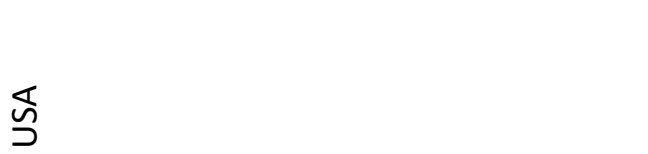 \\
\hline & 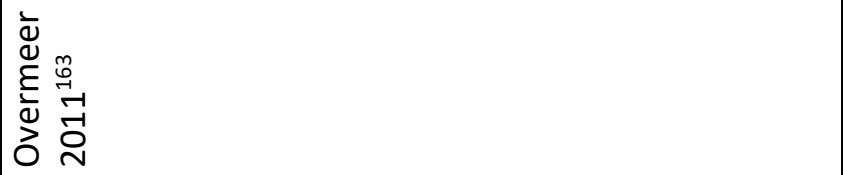 & 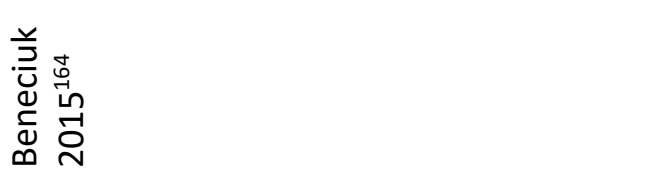 \\
\hline
\end{tabular}




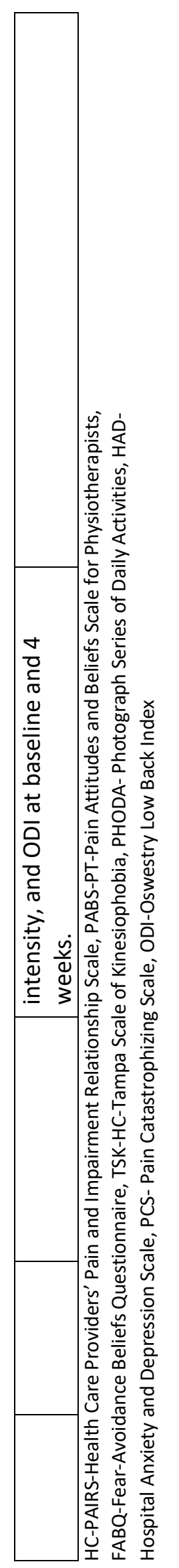




\section{Chapter 3: Methodology}

\section{Introduction}

This chapter describes the methods utilized in this dissertation. The chapter begins with a detailed explanation of the study design and procedures. This is followed by information about the instruments that were used to collect data from physical therapists. Next, a detailed description of the outcomes data and data analysis is included. Lastly, the limitations and delimitations of the study are discussed.

\section{$\underline{\text { Study Design }}$}

This project is a retrospective cohort design that included a survey of physical therapists and the collection of patient outcomes from Focus on Therapeutic Outcomes, Inc. (FOTO). The survey was performed electronically using the Research Electronic Data Capture (REDCap) tool. A link for the survey was generated by REDCap and provided to FOTO. The research facilitation team at FOTO delivered the link, via e-mail, to FOTO clinic contacts and FOTO users who opted to receive marketing e-mails from the company. Users were asked to distribute the link to all registered FOTO users at their clinic. Survey participants provided their name, email address, state, and clinic name, which was used by FOTO to extract patient outcome data. To encourage participation, a financial incentive in the form of a random drawing was offered to participants.

The survey was sent by FOTO on 2/6/19, 2/26/19, and 3/28/19. Data collection was stopped on 4/24/19 and the participants' names, email addresses, states, and clinic names were sent to the research facilitation team at FOTO. The outcomes data of patients with low 
back pain (LBP) treated by the physical therapists who completed the survey were queried for 2017 and 2018. Data from 2018 were used for the primary analysis and 2017 data were used for cross validation. Due to privacy concerns, FOTO was not able to provide data on individual patients. Instead, FOTO provided minimum, maximum, and average patient data for each participating physical therapist. The patient data provided by FOTO was keyed based on a participant identification number. This data was merged with the physical therapist survey data using the participant identification number.

\section{Clinician Survey}

The clinician survey was created in REDCap and contained four sections. The first section collected demographic and other basic information about the physical therapists. The second and third sections of the survey measured physical therapist attitudes and beliefs about LBP using the Health Care Providers' Pain and Impairment Relationship Scale (HC-PAIRS) ${ }^{10}$ and the Pain and Impairment Relationship Scale for Physiotherapists (PABS-PT). ${ }^{136}$ The final section of the survey measured physical therapist knowledge of pain mechanisms using the Neurophysiology of Pain Questionnaire (NPQ). ${ }^{174}$

\section{Section One}

The survey questions utilized in this section can be found in the appendix. These include questions on age, sex, ethnicity, years of experience, entry-level and advanced education, experience treating patients with LBP, practice setting, and personal experience with LBP (see the appendix). These questions were used to describe the study sample and to provide insight into factors that are associated with the attitudes and beliefs of clinicians. Several previous 
studies have attempted to identify factors that are associated with, and potentially influence the development of, clinician attitudes and beliefs about LBP. ${ }^{45,63,142,143,145,146,151,170-173}$ Alshami et al. studied the attitudes and beliefs of student physical therapists in Saudi Arabia, Australia, and Brazil using the HC-PAIRS. ${ }^{173}$ They found no difference in HC-PAIRS scores based on sex, the year of school, personal history of LBP, or country. In a study of French general practitioners, Coudeyre et al also found no association between fear-avoidance beliefs (FABs) and clinician factors such as age, sex, years of practice, and personal history of LBP. ${ }^{142}$ In contrast, several other studies have found a relationship between clinician attitudes and beliefs and practice setting, ${ }^{146}$ age,,$^{45,143}$ years of practice, ${ }^{45,63,171,175}$ and sex. ${ }^{171}$

\section{Section Two}

Several questionnaires have been used to measure the attitudes and beliefs of clinicians about LBP. ${ }^{62}$ The vast majority of these questionnaires are direct modifications of patientoriented scales. ${ }^{62}$ Commonly used patient self-report questionnaires such as the FearAvoidance Beliefs Questionnaire (FABQ), ${ }^{46,176}$ the Tampa Scale of Kinesiophobia (TSK), ${ }^{46,61}$ the Back Beliefs Questionnaire (BBQ), ${ }^{46}$ the Attitudes to Back Pain Sale (ABS), ${ }^{147}$ and the Pain and Impairment Relationship Scale (PAIRS) ${ }^{10}$ have been reworded to use with clinicians. The HCPAIRS and PABS-PT are two of the most commonly used questionnaires and are the most thoroughly tested instruments for the measurement of clinician attitudes and beliefs about LBP. ${ }^{62}$ As a result, they were used as the primary instruments in this study. 


\section{Health Care Providers' Pain and Impairment Relationship Scale}

The PAIRS was developed by Riley et al. in 1988 to measure the beliefs of patients with chronic pain about the relationship between pain and function. ${ }^{177}$ In 1995 , Rainville et al. modified the PAIRS to measure the beliefs of healthcare professionals. ${ }^{10}$ This was done by changing the subject of each item from "I" to "Chronic back pain patients." For example, the first question on the PAIRS is "I can still be expected to fulfill work and family responsibilities despite pain" and the first question on the HC-PAIRS reads "Chronic back pain patients can still be expected to fulfill work and family responsibilities despite pain." The scale contains 15 statements about LBP and respondents rate their agreement with the statement on a 7-point scale (see the appendix). The scale is scored from 1 (Completely Disagree) to 7 (Completely Agree), with 4 being a neutral response. Items 1, 6, and 14 are reverse scored. Scores can range from 15 to 105, with higher scores indicating a stronger belief that chronic back pain should result in disability. ${ }^{10}$

The original study by Rainville et al. identified four factors in the HC-PAIRS. These were functional expectation ( 8 items), social expectation ( 4 items), need for a cure (3 items), and projected cognition ( 2 items). ${ }^{10}$ These factors explained $29 \%, 10 \%, 9 \%$, and $8 \%$ of the variance respectively. Houben et al. also performed a confirmatory factor analysis on the HC-PAIRS and found that the original 4-factor model described by Rainville et al. did not fit the data. ${ }^{61}$ In a new factor analysis, Houben et al. found a 1-factor model was the best fit. ${ }^{61}$ Both Houben et al. and Rainville et al. found that questions 10 and 13 detracted from the internal consistency of the scale and Houben et al. excluded those two items from the final version of the scale. ${ }^{10,61}$ This left a 13-item scale that included items 1-9, 11, 12, 14, and 15, with potential scores 
ranging from 13-91. No other studies were identified that performed a factor analysis of the HC-PAIRS. Studies using the HC-PAIRS have consistently utilized a 1-factor model; ${ }^{54,163,176,178-180}$ however, the use of the 15 -item vs the 13 -item version varies throughout the literature. Given the improved internal consistency and reduced test burden, the 13-item version of the scale was utilized in this study.

Six studies have investigated the reliability of the HC-PAIRS. The majority of these studies include physical therapists, ${ }^{10,61,176,178,179}$ half of them used an English version of the scale, ${ }^{10,54,179}$ and two were performed on clinicians in the United States (US). ${ }^{10,54}$ Five of the six studies reported the internal consistency of the instrument. ${ }^{10,61,176,178,179}$ Internal consistency represents the homogeneity of the construct measured by the items of an instrument. ${ }^{181}$ Higher internal consistency suggests that the items of an instrument measure the same construct and lower values suggest that more than one construct is being measured. Internal consistency also provides insight into the amount of error that can be expected from an instrument. Higher internal consistency suggests that scores on an instrument include less error variance. ${ }^{182}$ The studies investigating the HC-PAIRS used Cronbach's coefficient (Alpha, $\left.\alpha\right)$ to measure internal consistency. Alpha ranges from 0 to 1 , with higher scores indicating greater correlation between the items of the instrument. ${ }^{181,182}$ Interpretation of alpha varies based on the length of the instrument and expectations of the tester; but, in general, authors suggest that values between 0.70 and 0.95 are acceptable. ${ }^{182}$ Scores lower than 0.70 suggest more than one construct is being measured and scores higher than 0.95 suggest redundancy between items. ${ }^{182}$ Internal consistency of the HC-PAIRS ranges from a low of $\alpha=0.71$ for a 15 -item Brazilian-Portuguese version ${ }^{178}$ to a high of $\alpha=0.92$ for the 13 -item English version. ${ }^{179}$ Only one 
study investigating internal consistency included US physical therapists. ${ }^{10}$ This study, by Rainville et al., included 150 healthcare workers with 34\% being physical therapists. With the removal of items 10 and 13 , the alpha score of the HC-PAIRS was 0.78 . This finding was replicated by Houben et al., who also found that the deletion of items 10 and 13 resulted in improved internal consistency $(\alpha=0.83)$. Another study that included physical therapists and osteopathic physicians from New Zealand found high internal consistency $(\alpha=0.92)$ for the 13item version of the HC-PAIRS. ${ }^{179}$

Test-retest reliability measures the stability of an instrument with repeated administration. ${ }^{181}$ Instruments with greater error will have more variability when repeated on subjects who are unchanged. Several statistical tests are available for assessing the reliability of an instrument. Both the Spearman rho test and the Intraclass correlation coefficient (ICC) can be used to assess the reliability of ordinal scale measures. The ICC is the preferred test because it includes both correlation and agreement. ${ }^{181}$ Generally, when ICC scores are $>0.5$, test-retest reliability is considered poor, for scores between 0.5 and 0.75 , test-retest is considered moderate, for scores between 0.75 and 0.9 , test-retest is considered good, and for scores above 0.9 , test-retest is considered excellent. ${ }^{181,183}$ Four studies have investigated the testretest reliability of the HC-PAIRS. ${ }^{54,176,178,179}$ Two of the studies included physical therapists, ${ }^{178,179}$ one included student physical therapists, ${ }^{176}$ and one tested reliability in physicians. ${ }^{54}$ The study including student physical therapists was performed on a Spanish version of the HC-PAIRS and the 4-week test re-test reliability was moderate $(r=0.50) .{ }^{176}$ Magalhaes et al. found good reliability $(r=0.84)$ for a Brazilian version of the HC-PAIRS tested 
over a 7 day period. ${ }^{178}$ These results were confirmed by Moran et al., who also found good testretest reliability $(r=0.84)$ for an English version of the scale. ${ }^{179}$

Two of the studies investigating the reliability of the HC-PAIRS reported the Standard Error of Measurement (SEM), a group of tests that assess measurement error. ${ }^{184}$ In the studies investigating the HC-PAIRS, SEM represented the standard deviation of the measurement error. ${ }^{181}$ This was calculated using the standard deviation of the sample and a reliability coefficient, which is estimated using test-retest reliability scores from previous research $\left(\mathrm{SEM}=\mathrm{S}_{\mathrm{x}} \mathrm{V} 1-\mathrm{r}_{\mathrm{xx}}\right) .{ }^{181}$ Lower values for $\mathrm{SEM}$ suggest less error variance and can be interpreted similarly to a standard deviation value. The SEM for the HC-PAIRS was 3.75 for a sample of 91 Osteopaths and 35 PTs from New Zealand, ${ }^{179}$ and 4.34 for a sample of 100 Brazilian student physical therapists.

Responsiveness is the ability of an instrument to detect change. ${ }^{181} \mathrm{Greater}$ responsiveness suggests that an instrument has the ability to identify smaller changes in the measured construct. The responsiveness of the HC-PAIRS has been studied by investigating changes in the instrument after clinicians are exposed to an educational intervention that is expected to influence attitudes and beliefs. For example, Domenech et al. found an average drop in HC-PAIRS scores of 23 points in student physical therapists who were given training on biopsychosocial factors. ${ }^{176}$ Several other studies found changes in HC-PAIRS scores after education ${ }^{44,180,185-187}$ with average change scores ranging from $0.88^{180}$ to $17.5{ }^{187}$ Two studies found that the HC-PAIRS could differentiate between students who were just starting their education and those towards the end of their entry-level training. ${ }^{125,188}$ In contrast, two studies found no changes in HC-PAIRS scores in physical therapists ${ }^{189}$ and students ${ }^{168}$ who participated 
in six hours and three hours of biopsychosocial focused training, respectively. Interpreting the results of these studies is difficult because the actual degree of change in attitudes and beliefs after an educational intervention is unknown. In general, HC-PAIRS scores change, in the expected direction, when subjects participate in education targeting attitudes and beliefs. This suggests that the HC-PAIRS can detect some degree of change in attitudes and beliefs about LBP.

The construct validity of the HC-PAIRS has been investigated by eight studies. Construct validity represents the instrument's ability to measure the paradigm it purports to measure. ${ }^{181}$ Measuring the construct validity of an instrument is complex because it is related to an abstract concept that often has no definitive anchor. ${ }^{181,190}$ The HC-PAIRS is designed to measure a clinician's beliefs about the connection between LBP and function. There is currently no commonly accepted reference standard for this construct. As a result, indirect methods of evaluation have been used to assess validity. These studies have utilized four main approaches.

The first approach is to compare the HC-PAIRS scores of practitioners who are expected to have different beliefs about LBP and function. If the HC-PAIRS is able to differentiate between providers who have different beliefs, it suggests that the instrument is measuring the proper construct. This approach was taken by Rainville et al., who compared the HC-PARIS scores of 150 healthcare workers (including physical therapists) with 66 functional rehabilitation experts. ${ }^{10}$ Functional rehabilitation experts were clinicians who worked in treatment programs that focused on impairments, function, and patient attitudes and beliefs. These programs put less emphasis on symptoms and the authors expected that providers working in this setting would be less likely to see a strong connection between symptoms and 
function. As anticipated by the authors, clinicians with a functional rehabilitation background scored lower on the HC-PARIS (14 points, $p>0.001$ ), suggesting that the HC-PAIRS has construct validity.

The second approach to investigate the construct validity of the HC-PARIS has been to compare the activity recommendations of healthcare workers to questionnaire scores.

Clinicians who score higher on the HC-PAIRS are expected to have stronger beliefs that LBP results in lower functional activity tolerance. These lower functional expectations are expected to influence the type of advice a clinician provides to patients. Three studies have investigated the relationship between HC-PAIRS scores and the recommendations provided by various healthcare workers. ${ }^{54,61,176}$ Rainville et al. analyzed the activity recommendations of 82 physicians from the US (spine surgeons $n=41$, family practitioners $n=41$ ). ${ }^{54}$ These physicians filled out the HC-PAIRS and answered questions about work and activity recommendations based on three patient vignettes. The physicians were asked 4 questions for each vignette, including the severity of the symptoms, amount of spinal pathology, suggestions for maintaining physical activity despite the pain, and recommendations for returning to work. HCPAIRS scores correlated with physician activity $(r=0.37, p=0.001)$ and work recommendations for vignette $1(r=0.37, p=0.001, r=0.29, p=0.01)$ and for vignette $3(r=0.30, p=0.006, r=0.29$, $p=0.01)$. This weak correlation suggests that higher scores on the HC-PAIRS are associated with more restrictive activity and work recommendations. Houben et al. performed a similar study on physical therapists, chiropractors, manual therapists, and McKenzie therapists from the Netherlands. ${ }^{61}$ Participants were asked to fill out the HC-PAIRS and then answer the same vignette questions used in the Rainville et al. study. HC-PAIRS scores were correlated with 
recommendations for all three vignettes in the expected direction ( $r=0.25$ to $0.45, p<0.002$ ). Domenech et al. used the same methodology on 174 student physical therapists and 32 general practitioners from Spain. ${ }^{176}$ Unlike the Rainville and Houben studies, Domenech et al. did not find a correlation between HC-PAIRS scores and the activity recommendations of students or a combination of students and general practitioners. There was a moderate correlation between recommendations and HC-PAIRS when a sub-analysis of just the general practitioners was performed.

The final method used to investigate the construct validity of the HC-PARIS has been to compare HC-PARIS scores with scores on other questionnaires that purport to measure the same or similar construct. If the scores on these questionnaires are correlated with the HCPAIRS, it suggests that they are measuring a similar construct (convergent validity). Five studies that compare the HC-PAIRS to other instruments were identified. ${ }^{46,61,176,178,179}$ All of these studies found that HC-PAIRS scores were correlated with the other measures of clinician beliefs. Houben et al. compared the HC-PARIS scores of 295 Dutch physical therapists to their scores on the PABS-PT. ${ }^{46}$ They found a moderate correlation between HC-PAIRS and the subscales of the PABS-PT in the expected directions $(r=0.517, p<0.001, r=-0.472, p<0.001)$. Magalhaes et al. compared the HC-PAIRS and PABS-PT scores of 100 Brazilian physical therapists. ${ }^{178}$ Unlike the Houben study, the correlation between the two scales in the Magalhaes study was weak ( $r=$ $0.19, p=0.055, r=-0.28, p=0.005)$. Two studies compared HC-PAIRS scores to scores on the FABQ. One study included student physical therapists and general practitioners from Spain ${ }^{176}$ and the other included physical therapists and osteopathic physicians from New Zealand. ${ }^{179}$ Convergent validity was found to be good in both studies, with correlations ranging from 0.557 
to 0.70 . Houben et al. ${ }^{61}$ and Moran et al. ${ }^{179}$ also found a moderate to strong correlation between the HC-PAIRS and the TSK (0.62-0.69).

\section{The Pain Attitudes and Beliefs Scale for Physiotherapists}

The PABS-PT was developed in 2003 by Ostelo et al. ${ }^{11}$ The goal of the instrument was to measure the attitudes and beliefs of physical therapists about the development and maintenance of chronic LBP. ${ }^{178}$ The scale was designed on the premise that healthcare providers have attitudes and beliefs that are aligned with a biomechanical or biopsychosocial model. ${ }^{136}$ The intent was to develop a scale that could differentiate between providers with biomedical-oriented attitudes and beliefs and those with biopsychosocial-oriented attitudes and beliefs. The PABS-PT was created by modifying questions from patient-oriented questionnaires such as the TSK ( 8 items), the PCS, the BBQ ( 2 items) and the FABQ ( 2 items). ${ }^{11}$ Additional questions that were considered relevant to the treatment of LBP were also added (19 items). These 37 items were submitted to a panel of experts who shortened the tool to 31 items. The items consist of statements such as "good posture prevents back pain" and "if back pain increases in severity, I immediately adjust the intensity of my treatment accordingly." Respondents are asked to rate their agreement with each statement on a 6-point scale. Scores of 1-3 indicate disagreement with the statement and include, totally disagree (1), largely disagree (2), and disagree to some extent (3). Ratings 4-6 correspond with agree to some extent (4), largely agree (5), and totally agree (6).

Factor analyses were performed on the scale in several studies. ${ }^{46,136,150,151,178,191,192}$ All of the studies found two factors (biomedical, biopsychosocial); however, there is disagreement 
about the items that make up each factor. In the original development study, Ostelo et al. included 14 items and 6 items in the two factors. ${ }^{136}$ The biomedical factor (14 items) had a Cronbach's alpha of 0.84 and the biopsychosocial factor had an alpha of 0.54. In an attempt to improve the internal consistency of the second factor, Houben et al. added five additional questions to the scale. ${ }^{46}$ They identified a 10 -item biomedical factor and a 9-item biopsychosocial factor. Nine of the items included in the first factor overlapped with items in the original Ostelo factor. One of the items in the new biomedical factor was from the five items that were added by Houben et al. The biopsychosocial factor included five of the items in the original Ostelo factor, two of the five items added by Houben, and two items from the original 31 items that were not included in the Ostelo factor. The internal consistency of the new biomedical factor remained good $(\alpha=0.80)$ and the biopsychosocial factor's internal consistency improved to 0.68 . Watson et al. performed a factor analysis of the PABS-PT in 85 British general practitioners. ${ }^{151}$ Using the factors developed by Houben et al., Watson found an alpha of 0.781 and 0.296 for the two factors. The internal consistency of the factors improved when one item was eliminated from factor one and 4 items were removed from factor two. This resulted in a 9-item biomedical factor $(\alpha=0.790)$ and a 5-item biopsychosocial factor $(\alpha=0.602)$. Laekeman et al. translated the PABS-PT into German and performed a factor analysis based on responses from German physical therapists and student physical therapists. ${ }^{150}$ They identified a biomedical factor with 10 items and a 4-item biopsychosocial factor. Seven of the items in the first factor aligned with the Houben version and three of the items in the second factor were also include in the Houben version. Internal consistency for the German version was found to be 0.77 and 0.58 . A factor analysis on the Norwegian version of the PABS-PT 
identified a 13-item biomedical and a 6-item biopsychosocial factor. ${ }^{191}$ Ten of the 13 items in factor one were included in the Houben version and all 6 of the items in factor two were contained in the Houben version. The internal consistency of factor one was good $(\alpha=0.79)$ and, consistent with other studies, the second factor had lower internal consistency $(\alpha=0.57)$.

For this study, the 19-item Houben version of the PABS-PT was utilized. This version contains a 10-item biomedical and a 9-item biopsychosocial factor. It was developed using scores from 295 physical therapists and has acceptable internal consistency for both factors. The Houben version of the PABS-PT is widely used in the literature $44,45,138,146,163-165$ and has significant overlap with the other versions of the scale.

Three studies have examined the reliability of the PABS-PT and have found it to be acceptable. ${ }^{150,178,192}$ Bowey-Morris examined the test-retest reliability of the scale in 83 general practitioners in the United Kingdom. ${ }^{192}$ The mean difference between scores for factor one was -0.15 points and factor two was -0.35 . The biomedical factor had good reliability $(0.81)$ and the biopsychosocial factor had moderate reliability (0.65). Laekeman et al. studied the reliability of a German version of the PABS-PT in 70 general practitioners and 30 student physical therapists. ${ }^{150}$ After five weeks, the reliability was 0.83 for the first factor and 0.70 for the second factor. Magalhaes et al. found similar results in a Portuguese version of scale, with an ICC of 0.80 for the biomedical factor and 0.70 for the biopsychosocial factor. ${ }^{178}$ Bowey-Morris and Magalhaes et al. reported an SEM of $3.13^{192}$ and $3.57^{178}$ for the first factor and $1.47^{192}$ and $3.48^{178}$ for the second factor. 
The responsiveness to change of the PABS-PT has been investigated in physical therapists and general practitioners. ${ }^{44,165,192}$ The three identified studies found that PABS-PT scores changed in the expected direction after education. For example, two studies looked at changes in scores after physical therapists participated in a course that covered biopsychosocial topics. ${ }^{44,165}$ Vonk et al. found that Dutch physical therapists who attended a 2-day training session had an average reduction in biomedical scores of 4.4 points and an average increase of 0.67 in biopsychosocial scores. ${ }^{165}$ Overmeer et al. found an increase in the biopsychosocial score $(2.1$ points, $\mathrm{P}<0.004))$ and reduction in the biomedical score $(8.1, \mathrm{P}<0.001)$ in 42 Swedish physical therapists who completed a course focused on psychosocial prognostic factors. ${ }^{44} \mathrm{~A}$ third study looked at PABS-PT changes in 73 UK-based general practitioners who took a 2-hour course on the management of non-specific LBP. The study found an 8.88-point drop in the biomedical factor and a 2.44-point increase in the biopsychosocial factor. ${ }^{192}$

Similar to the HC-PAIRS, there is no specific anchor that can be used to determine the validity of the PABS-PT. Instead, construct validity has been tested by comparing scores from various providers, by comparing PABS-PT scores to expected clinical behaviors, and by looking for concurrent validity with other measures. $46,48,63,136,138,150,151,178$ Studies have consistently found that PABS-PT scores can differentiate between clinicians with divergent attitudes and beliefs, ${ }^{136}$ are correlated with clinical behaviors, ${ }^{46,48,63,150}$ and have convergent validity with other measures. $46,138,150,178$

Ostelo et al. looked at the impact of various clinician characteristics on PABS-PT scores. ${ }^{136}$ They surveyed 421 physical therapists from the Netherlands and asked them to identify their specialization and the education courses they have attended. Self-reported 
specialization was coded as either biomedical or biopsychosocial. Physical therapists identifying as manual therapists or McKenzie therapists were coded biomedical $(n=89)$ and those identifying as chronic pain specialists were coded biopsychosocial $(n=15)$. A similar approach was used to code education courses. Courses focused on manual therapy were coded biomedical $(n=61)$, and courses focused on topics such as cognitive behavioral strategies and graded exposure were coded as biopsychosocial $(n=34)$. Scores on the PABS-PT correlated with specialization and education in the expected direction. Physical therapists coded as biomedical specialists had statistically significant higher scores ( 41.2 vs. $31.9, p=0.002)$ on the biomedical factor and those who only took biomedically-oriented courses scored lower on the biopsychosocial scale (18.4 vs. $21.4, p=0.002)$.

Four studies have found a correlation between PABS-PT scores and clinical behavior. ${ }^{46,48,63,150}$ As expected, clinicians who score higher on the biomedical factor tend to give recommendations that are more restrictive of physical activity and those who score higher on the biopsychosocial factor recommend more physical activity. This trend was seen in a study of 295 physical therapists from the Netherlands. ${ }^{46}$ Participants were asked to give physical activity recommendations based on three patient vignettes. There were moderate to weak statistically significant correlations between PABS-PT scores and activity recommendations in the expected direction. Bishop et al. had similar results in 442 general practitioners and 580 physical therapists from the United Kingdom. ${ }^{48}$ In this study, the authors categorized advice to restrict physical activity as divergent from guideline recommendations and advice to stay active was considered aligned with guidelines. Of the clinicians whose work recommendations deviated from the guidelines, $45 \%$ had high biomedical and low biopsychosocial scores and only 
$12 \%$ had low biomedical and high biopsychosocial scores. There was also a linear relationship between divergent advice and scores on the two factors, in the expected direction. Two other studies involving physical therapists from Germany ${ }^{150}$ and general practitioners from Ireland ${ }^{63}$ also found expected correlations between PABS-PT scores and clinical behavior.

The PABS-PT has been found to have convergent validity with the TSK, BBQ, HC-PAIRS and Photograph series of Daily Activities harmfulness ratings (PHODA). ${ }^{46,138,150,178}$ Houben et al. published 2 studies in 2005 which examined the correlation between the PABS-PT and several other questionnaires that measure clinician attitudes and beliefs. ${ }^{46,138}$ They found that scores on the PABS-PT correlated with the HC-PAIRS, the BBQ, PHODA, and the TSK. The TSK had the strongest correlation $(0.79, \mathrm{p}<.001$, with the biomedical factor) and the weakest statistically significant correlation was between the HC-PAIRS and the biomedical factor $(.35, p<.05)$. Other authors found that the factors of the PABS-PT correlated with the TSK $(0.72,-0.54)^{150}$ and the HC-PAIRS $(0.28,-0.19) \cdot{ }^{178}$

\section{Section Three}

\section{Neurophysiology of Pain Questionnaire}

Moseley developed the NPQ in 2003 to the measure the pain neurophysiology knowledge of healthcare professionals and patients. ${ }^{193}$ The scale was initially created by selecting 30 items from examinations that were given to medical students. Eleven items were eliminated because they were either redundant (1) or not appropriate for both clinicians and patients (10). The final scale consisted of 19 questions rated as either "True", "False," or

"Undecided." A total score between 0-19 is calculated by summing the number of correct 
answers. In 2013, Catley et al. performed a Rash analysis on the scale and recommended the elimination of 7 questions. ${ }^{174}$ This revised scale contains 12 true/false items, with scores ranging from 0 to 12 .

The psychometrics of the NPQ have not been extensively studied. The original article by Mosely et al. examined the construct validity of the scale. ${ }^{193}$ They found that professionals who completed a group education session and patients who completed a one-on-one education session scored better than their untrained counterparts $(p<0.005)$. Catley et al. found that the NPQ had a person separation index of 0.84 , which indicates good internal consistency. Catley et al. also found that the NPQ had good test-retest reliability in patients. A French version of the scale was found to have modest internal validity $(\alpha=0.44)$ and good reliability $(0.644)$ in patients with chronic spine pain. ${ }^{194}$ Meeus et al. studied the test properties of a Dutch version of the NPQ in 61 healthy individuals with chronic pain and 31 healthcare professionals (physical therapists and physicians). ${ }^{195}$ The reliability of the Dutch version was good (ICC=0.76) and the internal consistency was acceptable ( $\alpha=0.77)$. Despite the paucity of studies examining the psychometric properties of the NPQ in clinicians, it has been commonly used to measure pain neurophysiology knowledge in healthcare professionals. ${ }^{168,196-198}$ In this study, the revised NPQ was used as a secondary measure to determine the pain neurophysiology knowledge of physical therapists.

\section{Patient Outcome Data}

Patient information was obtained from the FOTO database. FOTO is an international commercial outcomes assessment system that is primarily used by physical therapists to 
measure the physical function of patients with musculoskeletal conditions. FOTO is used in 5,012 clinics and includes over 22,000 clinicians. ${ }^{199}$ The database contains nearly 22 million patient assessments and 6 million completed episodes of care. FOTO has several standard measures that are body part or condition specific. These standard measures are collected from every patient who has a particular condition or affected body region. For example, every patient entered into the system with LBP will be given the "Lumbar/Low Back Pain" instrument. Every patient also fills out a basic demographic questionnaire, the FABQ-physical activity, and a measure of patient satisfaction. The database also includes several optional surveys that include the PCS, STaRT Back Screening Tool, FABQ-work, Oswestry Disability Index, and specific questions about pain. The standard orthopedic measures, including the LBP measure, are computer adaptive tests (CAT). CATs utilize an algorithm that adjusts the difficulty of the questions presented to a patient based on their performance on previous questions. If a patient scores high on a question, subsequent questions will be at a higher level. This allows the measurement of a wider range of a construct with fewer questions. As a result, CATs have fewer problems with floor and ceiling effects while minimizing the testing burden on patients.

FOTO instruments are collected utilizing a patient inquiry computer-based software that FOTO developed. FOTO recommends that patients enter demographic information and complete the appropriate standard measures for their condition at the initial encounter with their physical therapist. Patients may also complete optional measures if deemed appropriate by the physical therapist. The standard functional measures ask patients a series of questions about the amount of difficulty they have with various activities. Based on the answers provided by the patient, the patient inquiry software calculates a total functional status score that ranges 
from 0 (low functioning) to 100 (high functioning). Ideally, these measures are regularly repeated throughout the course of treatment and at the final visit. At the final visit, patients also complete a satisfaction survey, and information, such as the number of visits and final date of service, are entered.

\section{Computerized Lumbar Functional Status Measure}

The primary measure of patient outcomes in this study was the Computerized Lumbar Functional Status measure (CLFS) included in the FOTO database. The CLFS is designed to measure the functional status of individuals with LBP. The original, non-computerized, version of the CLFS was first described by Stratford et al. ${ }^{200}$ The authors reviewed other questionnaires, gathered information from 63 patients via the Patient Specific Functional Scale, and interviewed six experienced physical therapists. This process identified 74 items to be considered for inclusion in the instrument. The list was reviewed for redundant items and was condensed to 25 items. The items were then administered to 96 patients and reduced to 12 items based on factors such as item means, item option endorsement frequency, and internal consistency. ${ }^{200}$ Hart et al. took the original 25 items of the lumbar functional status measure and developed a computer adaptive version based on Item Response Theory. ${ }^{201}$ The computer adaptive version of the lumbar functional status measure utilized an average of seven questions to reach the same accuracy as the 25 -item paper version. This constituted a $72 \%$ increase in efficiency. The question stem for all items in the CLFS is, "Today, because of your back problem, do you or would you have any difficulty at all performing." The patient is then presented with a list of activities, such as "usual work," "lifting from the floor," or "walking 1 mile." Patients rate 
their ability to perform each activity on a 0-6 scale, with 0 meaning "unable to perform" and 6 equating to "no difficulty."

Two studies investigating the reliability of the CLFS were identified. Both studies indicate that the CLFS is a reliable measure of physical function in patients with LBP. ${ }^{200,202}$ Stratford et al. gave the paper version of the CLFS to 77 patients with LBP. ${ }^{200}$ The measure was repeated 48 hours later in 28 patients resulting in an ICC of 0.88 . The internal consistency was good ( $\alpha=0.93$ ) and the instrument had an SEM of $6.5 \%$. The second study reporting on the reliability of the CLFS was performed by FOTO. ${ }^{199}$ In this report, 262 patients with chronic musculoskeletal conditions filled out functional measures on two subsequent treatment visits. Fifty-seven of the participants filled out the CLFS and it was found to have excellent reliability ( $r=0.93)$.

Three studies investigating the validity of the CLFS were identified. ${ }^{200,201,203}$ The largest of these was performed on 17,439 patients with LBP. ${ }^{201}$ In this study, the CLFS's ability to discriminate between patients with known risk factors was used to investigate the relative validity of the CLFS. As expected, older patients, patients with a history of surgery, and patients with more comorbidities scored lower on the CLFS. This relative validity was confirmed in a follow-up study of 8,198 patients with LBP. ${ }^{203}$ In this study, CLFS scores were correlated with symptom acuity, surgical history, age, payer, and comorbidities, in the expected direction. When compared to the Oswestry Disability Index, the CLFS was a better predictor of all factors except payer. ${ }^{203}$ The CLFS was also found to have good convergent validity $(r=0.79)$ with the Roland-Morris Questionnaire in 77 patients, ages 18-79, who were attending physical therapy for LBP. ${ }^{200}$ 
One study investigating the responsiveness of the CLFS was identified. ${ }^{204}$ This study included 17,439 patients with LBP from 377 clinics. Only $38 \%(6,607)$ of patients included in the study had both an intake and discharge measurement on the CLFS. Responsiveness based on a statistical approach (minimal detectable changes (MDC)) and an anchor-based approach (minimal clinically important difference (MDIC)) were reported. The MDC was calculated using the $95 \%$ confidence interval and SEM values for 10 scale ranges $(0-10,11-20,21-30$ etc.). The authors reported an overall MDC for the scale and 10 specific MDCs for the various score ranges. The MDIC was anchored using a 3-point change on a 15-point global rating of change score. MDIC values were calculated using receiver operating characteristic analysis for each quartile. The overall average MDC for the CLFS was 13.9. The highest MDC (31.5) occurred for scores falling between 0-10 and 91-100, and the lowest MDC (6.0) was for scores between 4150. Global rating of change scores were available for 2,612 of the 17,439 patients. Eighty-three percent were categorized as improved based on a 3-point or greater change. The $\mathrm{MDCl}$ was $\geq 9$, $\geq 5, \geq 3$, and $\geq 5$ for the first through fourth quartiles respectively.

CLFS data was obtained from FOTO in two formats. The first format was an average change score for each physical therapist. This was calculated by subtracting the initial CLFS score from the discharge CLFS for each patient seen by a participating physical therapist. Patients without a discharge CLFS score were not included in the analysis. The CLFS change scores for each patient seen by a participating physical therapist were averaged by FOTO. The second format of CLFS scores obtained from FOTO were average residual scores. Residual scores were calculated based on a risk stratification model developed by FOTO. Risk stratification uses patient characteristics to equalize the risk of a poor outcome for each 
patient. This stratification allows for a more accurate ranking of clinicians and a more detailed assessment of other non-patient factors. ${ }^{205}$ Risk adjustment is commonly used in the literature and has been studies by several authors. ${ }^{205-210}$ For example, Rodeghero et al. found that patients with a high risk of a poor prognosis were older, had a longer symptom duration, more extensive surgical histories, were using medication, had lower baseline disability, and were more likely to have worker's compensation, no-fault or litigation as a payer source. ${ }^{208} \operatorname{In} 2018$, Burgess et al. performed a systematic review on risk stratification models used for patient reported outcome measures. ${ }^{211}$ Fourteen studies were identified including eight studies utilizing the FOTO database. They found that the most common variables were baseline outcomes scores, age, sex, comorbidities, symptoms duration, and surgical history. The risk stratification model developed by FOTO includes intake CLFS scores, age, gender, acuity, payer type, surgical history, exercise history, medication use, previous treatment, postoperative status, and the number of comorbidities. ${ }^{212}$ Based on these factors, FOTO predicts an expected value for the change in CLFS score at discharge. The predicted CLFS change score is subtracted from the actual CLFS change score for each patient. A residual score of zero indicates that the patient's outcome was equal to the predicted outcome based on the risk stratification model. Values greater than zero constitute better than predicted outcomes and values less than zero indicate lower than the expected outcomes. FOTO provided average residual scores for each participating physical therapist.

\section{Secondary Measures}

Average number of visits and Fear Avoidance Beliefs Questionnaire Physical Activity subscale (FABQpa) scores were used as a secondary dependent variable. Although not reported 
in the literature, it is reasonable to hypothesize that the LBP-related attitudes and beliefs of a physical therapist may influence decisions regarding the number of visits utilized for an episode of care. Although not a direct measurement, number of visits provides insight into the amount of resources used for an episode of care. FOTO provided the average number of visits for each participating physical therapist.

Changes in patient fear avoidance beliefs were measured using the FABQ and were included as a secondary measure. The FABQ was developed in 1993 by Waddell et al. to measure a patient's beliefs about the impact of LBP on physical activity and work. ${ }^{213}$ The questions were developed by Waddell et al. based on the work of Fordyce, Sandstrom and Esbjornsson. The questionnaire contains 16 statements about function and patients rate their agreement with those statements on a 7-point Likert scale. The scale ranges from 0 "Completely Disagree" to 6 "Completely Agree". Scores can range from 0-66 with higher scores indicating more fear avoidance. The FABQ has a work (FABQw) and a physical activity subscale. The FABQpa subscale is calculated by summing questions $2-5$ with scores ranging from 0 to 24 . The FABQw subscale is calculated by summing questions $6,7,9-12$, and 15 with scores ranging from 0 to 42. FOTO includes FABQpa as a standard measure for all patients seen for LBP and it was used in this dissertation.

The psychometric properties of the FABQ have been extensively studies and it is commonly reported in the literature. ${ }^{31,213-217}$ The test retest-reliability of the FABQpa ranges from 0.64 to $0.90^{214-216,218}$ and the internal consistence ranges from 0.52 to $0.79 .{ }^{214,218}$ The FABQpa was found to have a minimal detectable change of 8.95 and a standardized response mean of 0.82 (Cohen's d) in 123 patients with acute LBP. ${ }^{214}$ The validity of the FABQpa has been 
tested by comparing the scale to other measures and assessing its association with factors such as pain and disability. Swinkels-Meewisse et al. and Ostelo et al. both found a moderate to strong correlation between FABQpa and the TSK in patient with acute LBP. ${ }^{104,219}$ FABQpa scores are also correlated with Roland-Morris Disability Questionnaire scores, 220 pain visual analog scores, ${ }^{220}$ ODI scores, ${ }^{34,221}$ PCS scores, ${ }^{106,222}$ and Short Form-36 scores. ${ }^{34}$ The construct validity of FABQpa has also been supported by studies that found a connection between FABQ scores and the development of LBP, ${ }^{27}$ disability, ${ }^{8,97}$ pain intensity $8,105,106$ and prognosis. ${ }^{31,110,112}$ 


\section{Chapter 4: Results}

\section{Introduction}

This chapter will describe the data analysis and report the findings of the project. It will start by describing the process used to analyze the data and then report the results. The results section will be broken up into two main sections. The first section will report the results from the physical therapist survey. It will start by discussing general information on the responses and then describe the demographics of the participating physical therapists. Next, it will report on the simple and multiple linear regressions used to find characteristics that predict the low back pain (LBP) related attitudes and beliefs of physical therapists. The next section reports on the combination of data from the physical therapist survey and patient data obtained from Focus on Therapeutic Outcomes, Inc. (FOTO). It will start by describing general information from the patient data and then discuss the demographics of the patients included in the study. Next, it will report the simple and multiple linear regression, and simple and multiple logistic regression used to find physical therapist and patient characteristics that predict patient outcomes.

\section{Data Analysis}

Data analysis was performed using Excel (Microsoft, Redmond WA) and SPSS V25 (IBM, Chicago IL). Significance levels were set at $\alpha=0.05$ for all analyses. All analysis was performed by the PhD candidate with consultation from Gary Brooks PT, DrPH and Dongliang Wang, PhD. 


\section{Data Cleaning}

Physical therapist data was screened for missing values using frequency tables.

Participants with missing or incomplete HC-PAIRS and/or PABS-PT data were excluded from the study. Missing data for other fields was deleted pairwise when appropriate. Variables with missing data were screened for non-random omissions using the Missing Value Module in SPSS. ${ }^{223}$ T-tests were performed on HC-PAIRS, PABS-PT and NPQ means for subjects with and without missing data and there was no significant difference between the groups. The data was screened for outliers using a cut off of \pm 2.2 of the interquartile range. ${ }^{224}$

Patient outcome data from FOTO was sorted based on the treating physical therapists' identification (ID) number that was generated by the Research Electronic Data Capture system (REDCap) used to administer the physical therapist survey. This ID number was used by SPSS to merge the physical therapist and patient data. FOTO supplied average patient data scores for each physical therapist for 2017 and 2018. Primary analysis was performed on the 2018 data and the 2017 data was used for validation. FOTO only queried patient cases with complete data so there was no missing patient data. The patient data was screened for outliers using a cut off of \pm 2.2 of the interquartile range. ${ }^{224}$ The merged data set was split into three samples for analysis. Sample 1 included data for physical therapists who had at least 5 completed patient cases for the 2018 dataset. Sample 2 and 3 included data for physical therapists with at least 10 or 20 completed cases, respectively. 


\section{Descriptive Statistics}

Descriptive statistics were calculated for all patient and physical therapist demographic data. Mean dependent variable scores and NPQ scores were calculated based on physical therapist demographics. Kruskal-Wallis tests were used to assess differences in belief scores and NPQ scores based on physical therapist demographics. Mean values for all physical therapist and patient data were calculated and reported. Descriptive statistics for the patient data were calculated for all three samples $(5,10$, and 20 patient cut-offs). Average CLFS change scores and average CLFS residual scores were categorized for logistics regression and reported descriptively. Average CLFS change score categories were based on the MCID (5 points) of the scale. ${ }^{204}$ Initially, the data was placed into five categories: negative change ( $\geq-5$ points), no change (-4 to 4 points), minimal improvement (5-9 points), moderate improvement (10-14 points) and significant improvement ( $\geq 15$ points). There were no physical therapists with an average negative CLFS change and only one therapist with an average CLFS change in the no change category. As a result, the categories were compressed to minimal to no change $(<10)$, moderate change (10-14), and significant change ( $\geq 15$ points). Average CLFS residual scores were categorized as less than expected change $(<0)$, expected change $(0)$, or more than expected change $(>0)$. No physical therapists had an average residual change of 0 , so average CLFS residual score was dichotomized into less than expected and more than expected change. 


\section{Research Questions 1-4 (Attitudes, beliefs, and knowledge of physical therapists and associated factors.)}

Simple linear regression was performed to compare physical therapist demographic information with scores on the HC-PAIRS and PABS-PT. Assumption testing for normality and variance homogeneity was performed using the Shapiro-Wilk test and Levene's test, respectively. The Shapiro-Wilk test was significant for all beliefs measures, indicating skewness. Log-transformation was performed; however, the Shapiro-Wilk test was still significant. Visually, the untransformed beliefs histograms were close to normally distributed. Simple linear regression and multiple linear regression are robust to small violations of normality. ${ }^{223}$ Logtransformation is controversial ${ }^{225}$ and both statistically and visually, the transformation did not improve the normality of the data. As a result, the untransformed data was used for both the simple and multiple linear regression.

Multiple linear regressions were performed on HC-PAIRS scores and PABS-PT scores. Demographic information was used as predictor variables and were initially selected based on theoretical prediction, and the results of the simple linear regression. The first step of model building was to perform an all-possible subset regression. ${ }^{226,227}$ Predictors used in the final model development were selected from the all-possible subset regression using Akaike information criterion (AIC). ${ }^{228} \mathrm{~A}$ final model was then built using a backwards stepwise approach. Pearson correlation was used to screen for multicollinearity in the model predictors. There were no correlations between variables greater than .70, which suggest no violation of the multicollinearity assumption. ${ }^{223}$ Normality was tested using the Shapiro-Wilk test and 
visually using histograms. Q-Q plots, variance inflation factor, and P-P plots were used to test residual normality, multicollinearity, and homoscedasticity. ${ }^{223}$

\section{Research Question 5 (Are physical therapists' attitudes and beliefs associated with the outcomes of patients with low back pain)}

Simple linear regression using average CLFS change scores, average CLFS residual scores, and the average number of visits was performed for all three patient cut-off samples. Variable normality and variance homogeneity were tested using the Shapiro-Wilk test and Levene's test, respectively. As noted above, HC-PAIRS and PABS-PT scores were significant for the ShapiroWilk test, but were visually normal. Simple logistic regressions using average CLFS change scores and average CLFS residual scores was also performed. Receiver operator characteristic curves were produced for average CLFS change categories and average CLFS change residual categories for beliefs scores that were significant univariate predictors.

Multilevel multiple linear regression for average CLFS change scores, average CLFS residual scores and average number of visits was performed for all three patient cut-off samples. The regression's hierarchical structure had the number of patients seen by the therapist entered into the first block. The second block contained the potential patient and physical therapist related predictors. Potential predictors entered into the second block of the regression were initially selected based on theoretical prediction, and the results of the simple linear regression.

The first step of model building was to perform an all-possible subset regression. ${ }^{226,227}$

Predictors used in the final model development were selected from the all-possible subset regression using $\mathrm{AlC} .{ }^{228} \mathrm{~A}$ final model was then built using a backwards stepwise approach. 
Cross validation of the final model was performed using two methods. The first approach was to rebuild the final model using the physical therapists' data from 2017. The model was considered validated if the same predictors were significant for the models created from the 2018 and the 2017 data. The second approach to cross validation was to calculate correlations between predicted values and actual values. The model created using 2018 data was used to calculate predicted values in the 2017 data. Pearson correlation was calculated and correlations of $>0.5$ were considered poor-fair, scores between 0.5 and 0.75 were considered moderate, scores between 0.75 and 0.9 were considered good, and scores above 0.9 were considered excellent. ${ }^{181,183}$ Dependent variables were assessed for normality using the Shapiro-Wilk test. The presence of a linear relationship between the independent and dependent variables was assessed using scatter plots and Pearson correlation coefficient. Independent variables without a linear relationship with the dependent variable were not included in the model. After creation of the model, residual normality, multicollinearity, and homoscedasticity were assessed using Q-Q plots, variance inflation factor, and P-P plots, respectively.

A multilevel multiple logistic regression was used to develop a predictive model for average CLFS change and average CLFS residual scores for all three cut-off samples. The regression's hierarchical structure had the number of patients seen by the therapist entered into the first block. Predictors included in model development were chosen based on theoretical prediction, and the results of the simple linear regression. A backwards stepwise approach was used to build the final model. 


\section{Provider Data Findings}

The provider survey was electronically sent to Focus on Therapeutic Outcomes, Inc. (FOTO) clients on 2/6/19 with two follow-up e-mails on 2/26/19 and 3/28/19. The individuals receiving the e-mails included all FOTO clinic contacts and FOTO clients who opted to receive marketing e-mails from the company. FOTO clinical contacts are clinic administrators and includes both physical therapists and non-clinical staff. At the time of the survey request FOTO had 23,285 registered clinicians in 5,012 clinics.

At the close of data collection on 4/24/19, 199 responses were recorded. Five respondents did not proceed past the consent page of the survey and 44 completed the consent page but did not respond to any other question. Of the remaining 150 participants, five did not complete the Health Care Providers' Pain and Impairment Relationship Scale (HC-PAIRS) or any items after that scale. All 145 respondents who started the HC-PAIRS, finished the scale with no missing items. The Pain Attitudes and Beliefs Scale for Physiotherapists (PABS-PT) was completed by 140 respondents and 1 subject partially completed the instrument. Data analysis was performed using the 140 respondents who completed both the HC-PAIRS and the PABS-PT questionnaires. This constitutes $70 \%$ of the initial 199 respondents and represents $0.6 \%$ of registered FOTO users.

Of the included subjects, seven had missing data on other survey items. Four respondents did not complete the Neurophysiology of Pain Questionnaire (NPQ) or the demographics sections of the survey. One subject completed all the items of the NPQ except item 6. An additional two subjects did not respond to the demographics and one participant 
had missing items. In total, five respondents had missing or incomplete NPQ responses (3.6\%) and six had missing or incomplete demographics (4.2\%). There was no statistically significant difference in mean HC-PAIRS, PABS-PT, or NPQ scores for subjects with and without missing data. Subjects with missing data were deleted listwise when appropriate.

\section{Physical Therapist Demographics}

Physical therapist demographics are reported in Table 4. Females made up $44.8 \%$ of respondents and $48 \%$ of respondents were between $25-34$ years of age. White, non-Hispanics accounted for $90 \%$ of participants and nearly $40 \%$ of respondents had over 20 years of experience. The most common entry-level degree was a doctorate (45.2\%), and $12.1 \%$ completed a transitional doctorate of physical therapy. Only 19 individuals were residency trained and 12 had completed a fellowship. Two participants were currently enrolled in a fellowship program and one was completing a residency. Thirty four percent were board certified through ABPTS and $12.9 \%$ were Certified Manual Therapists. Sixty eight percent of respondents reported at least a minimal change in their attitudes and beliefs about LBP over the past year. Research articles and courses were the most commonly cited factors that influenced the participants' change in beliefs. Ninety six percent of respondents reported a personal history of LBP, with $10 \%$ reporting that LBP had a significant impact on their life.

Descriptive statistics for belief scales and knowledge are reported in Table 5. HC-PAIRS scores ranged from 18 to 64 with a mean of 33.34, median of 33, and a mode of 36. PABS-BM scores ranged from 12 to 45 with a mean of 26.56 , median of 25.5 , and a mode of 25 . The range for the PABS-BPS was 27 to 48 with a mean of 36.47, a median of 37 and mode of 34 . NPQ 
descriptive statistics were calculated for 136 respondents. Values ranged from 2 to 12 with a mean of 9.4, median of 10 and a mode of 10 . No outliers were identified for the HC-PAIRS, PABS-PT or NPQ. The Shapiro-Wilk test was significant $(p<.05)$ for all belief measures suggesting a violation of normality. Histograms and Q-Q plots were inspected and a general normal trend was observed. Logarithmic transformation was performed and the Shapiro-Wilk test remained significant, and plots remained generally normal. Given the apparent normality on the histograms and Q-Q plots, the lack of improvement with transformation and the stability of simple and multiple regression with small violations of normality, transformed data was not used in the analysis. ${ }^{223}$ Non-parametric tests were utilized for comparing mean beliefs scores by demographics.

HC-PAIRS scores were less for White Non-Hispanics when compared to participants who selected "other" for race $(n=3)$, were lower for physical therapists who graduated with a Bachelor's degree versus those with an entry-level Master's or Doctorate, were less for respondents with a LBP case load of greater than $50 \%$ when compared to those with a case of less than $10 \%$, higher in participants who were not board certified in orthopedics and higher in participants who reported having no certifications. (Table 4) PABS-BM scores were lower for physical therapists who graduated with a Doctorate when compared to those graduating with a Bachelor's, lower for physical therapists who were board certified in orthopedics, less in respondents who completed a residency or fellowship, higher in physical therapists working in an in-patient setting, and higher in survey participants who do not strength train with free weights. Scores on the PABS-BPS scale were higher for 25-34 year olds when compared to 5564 year olds, lower for physical therapists who graduated with a Bachelor's degree when 
compared to those with entry-level Master's or Doctorate degrees, higher in respondents who have completed a residency or fellowship, higher in physical therapists board certified in orthopedics, and higher in those with any board certification when compared to non-board certified respondents.

Table 4. Mean belief and knowledge scores by demographic categories.

\begin{tabular}{|c|c|c|c|c|c|c|}
\hline & & & Mean Score & Group & & \\
\hline & $N$ & $\%$ & HC-PAIRS & $P A B S-B M$ & PABS-BPS & $\begin{array}{l}N P Q \\
(n=136)\end{array}$ \\
\hline All Respondents & 140 & 100 & $33.34(9.4)$ & $26.56(7.0)$ & $36.47(4.4)$ & $9.42(1.8)$ \\
\hline CURRENT AGE & & & & & & \\
\hline $18-24$ & 1 & 0.7 & 42.00 & 40.00 & 35.00 & 1 \\
\hline $25-34$ & 48 & 35.8 & $31.25(8.9)$ & $24.29(7.0)$ & $38.15(3.7)^{1}$ & $9.92(1.8)^{1}$ \\
\hline $35-44$ & 31 & 23.1 & $35.06(8.5)$ & $27.19(7.2)$ & $36.48(4.5)$ & $9.35(1.7)$ \\
\hline $45-54$ & 28 & 20.9 & $31.82(10.5)$ & $27.18(7.5)$ & $35.57(4.9)$ & $9.46(1.5)$ \\
\hline $55-64$ & 24 & 17.9 & $34.79(9.8)$ & $28.50(5.5)$ & $34.75(3.8)^{1}$ & $8.67(1.6)^{1}$ \\
\hline $65-75$ & 2 & 1.5 & $46.00(9.9)$ & $30.50(10.6)$ & $30.50(3.5)$ & $10.50(0.7)$ \\
\hline GENDER & & & & & & \\
\hline Male & 73 & 54.5 & $31.86(7.8)$ & $25.79(6.3)$ & $36.77(4.3)$ & $9.73(1.7)$ \\
\hline Female & 60 & 44.8 & $34.55(11.0)$ & $27.20(7.8)$ & $36.16(4.5)$ & $9.08(1.9)$ \\
\hline Abstain & 1 & 0.7 & 48.00 & 40.00 & 36.00 & 7.00 \\
\hline ETHNICITY/RACE & & & & & & \\
\hline White, Non-Hispanic & 121 & 90.3 & $31.93(7.9)^{1}$ & $25.96(6.5)$ & $36.71(4.3)$ & $9.50(1.8)$ \\
\hline Hispanic or Latino & 3 & 2.2 & $37.67(21.1)$ & $28.00(11.8)$ & $36.33(4.0)$ & $8.33(2.5)$ \\
\hline Asian or Pacific Islander & 2 & 1.5 & $50.50(10.6)$ & $31.00(9.9)$ & $31.00(0.0)$ & $8.00(4.2)$ \\
\hline Other & 3 & 2.2 & $54.33(9.0)^{1}$ & $37.00(6.6)$ & $33.33(1.5)$ & $8.00(3.0)$ \\
\hline Abstain & 5 & 3.7 & $41.20(13.2)$ & $31.40(12.5)$ & $35.00(5.6)$ & $9.60(1.5)$ \\
\hline
\end{tabular}




\begin{tabular}{|c|c|c|c|c|c|c|}
\hline \multicolumn{3}{|l|}{ Years of Experience } & \multirow[b]{2}{*}{$34.20(11.3)$} & \multirow[b]{2}{*}{$26.90(8.3)$} & \multirow[b]{2}{*}{$37.47(3.7)$} & \multirow[b]{2}{*}{$9.37(2.3)$} \\
\hline Less Than 4 & 30 & 22.4 & & & & \\
\hline $5-9$ & 25 & 18.7 & $30.32(8.3)$ & $23.88(6.8)$ & $37.64(4.3)$ & $9.76(2.0)$ \\
\hline $10-14$ & 11 & 8.2 & $35.36(8.3)$ & $25.18(4.1)$ & $35.27(5.4)$ & $10.00(1.5)$ \\
\hline $15-19$ & 15 & 11.2 & $32.93(7.6)$ & $26.67(7.4)$ & $37.67(4.3)$ & $9.73(1.5)$ \\
\hline $20-24$ & 17 & 12.7 & $35.29(10.2)$ & $28.53(9.4)$ & $36.53(5.0)$ & $9.12(1.4)$ \\
\hline 25 or more & 36 & 26.9 & 32.78(9.3) & $27.47(5.2)$ & $34.69(4.0)$ & $9.06(1.6)$ \\
\hline \multicolumn{7}{|l|}{ Entry-Level Degree } \\
\hline Bachelor's & 33 & 24.6 & $36.55(11.1)^{1,2}$ & $28.67(6.7)^{1}$ & $34.42(4.0)^{1,2}$ & $8.82(1.6)$ \\
\hline Master's & 37 & 27.6 & $31.49(7.0)^{1}$ & $26.62(7.1)$ & $37.19(4.6)^{1}$ & $9.48(1.4)$ \\
\hline Doctorate & 61 & 45.5 & $31.49(8.5)^{2}$ & $24.69(6.6)^{1,2}$ & $37.25(4.2)^{2}$ & $9.89(1.8)$ \\
\hline Other & 3 & 2.2 & $51.67(10.0)$ & $39.33(2.1)^{2}$ & $34.67(0.6)$ & $5.67(3.5)$ \\
\hline \multicolumn{7}{|l|}{ Post Graduate Education } \\
\hline None & 60 & 42.9 & $33.50(9.5)$ & $25.53(7.0)$ & $37.27(4.4)$ & $9.75(1.7)$ \\
\hline tDPT & 17 & 12.1 & $32.88(9.9)$ & $25.35(7.9)$ & $37.00(4.5)$ & $9.20(1.5)$ \\
\hline PhD & 1 & 0.7 & 53.00 & 38.00 & 33.00 & 11.00 \\
\hline DsC & 1 & 0.7 & 30.00 & 21.00 & 42.00 & 10.00 \\
\hline Residency & 19 & 13.6 & $30.00(6.0)$ & $23.47(6.0)^{1}$ & $38.95(3.8)^{1}$ & 10.11(1.9) \\
\hline Fellowship & 12 & 8.6 & $29.25(5.7)$ & $21.58(5.2)^{2}$ & $38.83(2.9)^{2}$ & $10.42(1.0)$ \\
\hline Other & 17 & 12.1 & $35.82(11.4)$ & $27.47(6.8)$ & $35.53(4.5)$ & $9.41(1.6)$ \\
\hline \multicolumn{7}{|l|}{ Certifications } \\
\hline None & 78 & 55.7 & $35.74(10.9)^{2}$ & $27.73(7.8)$ & $35.26(4.4)^{1}$ & $8.83(1.9)$ \\
\hline $\begin{array}{l}\text { Board Certified in } \\
\text { Orthopedics }\end{array}$ & 41 & 29.3 & $29.61(6.3)^{1}$ & $23.12(4.6)^{1}$ & $38.39(4.4)^{2}$ & $10.15(1.5)^{2}$ \\
\hline Board Certified in Sports & 2 & 1.4 & $35.50(0.7)$ & $22.00(2.8)$ & $42.50(0.7)$ & $10.50(0.7)$ \\
\hline Board Certified in Geriatrics & 0 & 0 & & & & \\
\hline Board Certified in Neurology & 1 & 0.7 & 39.00 & 28.00 & 36.00 & 10.00 \\
\hline Certified Manual Therapist & 18 & 12.9 & $33.06(9.0)$ & $27.00(7.6)$ & $36.33(3.9)$ & $9.94(1.8)$ \\
\hline
\end{tabular}




\begin{tabular}{|c|c|c|c|c|c|c|}
\hline Other Certification & 20 & 14.3 & $32.24(7.3)$ & $28.81(5.8)^{2}$ & $35.52(2.8)$ & $9.05(1.6)$ \\
\hline \multicolumn{7}{|l|}{ PRACTICE SETTING } \\
\hline $\begin{array}{l}\text { Private practice outpatient } \\
\text { orthopedics }\end{array}$ & 75 & 53.6 & $32.32(8.6)$ & $25.53(6.8)$ & $36.84(4.3)$ & $9.67(1.7)$ \\
\hline $\begin{array}{l}\text { Hospital-based outpatient } \\
\text { orthopedics }\end{array}$ & 43 & 30.7 & $33.53(9.1)$ & $27.56(7.0)$ & $35.47(4.8)$ & $9.19(1.6)$ \\
\hline $\begin{array}{l}\text { Rehab-based outpatient } \\
\text { orthopedics }\end{array}$ & 4 & 2.9 & 28.00(9.4) & $22.75(5.9)$ & $39.50(3.9)$ & $9.50(1.3)$ \\
\hline Other outpatient setting & 12 & 8.6 & $39.33(13.8)$ & $29.50(7.5)$ & $36.33(1.8)$ & $8.67(3.1)$ \\
\hline Inpatient setting & 6 & 4.3 & $34.83(5.6)$ & $31.83(5.4)^{1}$ & $33.67(2.9)$ & $9.17(2.1)$ \\
\hline Other setting & 4 & 2.9 & $42.75(5.6)$ & $35.50(5.5)$ & $35.75(2.9)$ & $9.50(1.3)$ \\
\hline \multicolumn{7}{|l|}{$\begin{array}{l}\text { Changes in LBP beliefs in the } \\
\text { last year }\end{array}$} \\
\hline No Change & 43 & 31.9 & $34.05(8.5)$ & 27.05(6.7) & $35.86(5.0)$ & $9.48(1.8)$ \\
\hline Minimal Change & 66 & 48.9 & $32.97(9.0)$ & $25.85(7.5)$ & $36.91(4.3)$ & $9.27(1.9)$ \\
\hline Significant Change & 26 & 19.3 & $32.04(12)$ & $27.04(7.0)$ & $36.85(4.0)$ & $9.73(1.7)$ \\
\hline \multicolumn{7}{|l|}{ LBP Case Load } \\
\hline Less than $10 \%$ & 8 & 6.0 & $40.63(8.1)^{1}$ & $30.50(5.5)$ & $35.12(3.2)$ & $8.50(1.6)$ \\
\hline $10-24 \%$ & 40 & 29.9 & $33.98(9.5)$ & $26.43(7.2)$ & $36.45(4.1)$ & $9.28(2.2)$ \\
\hline $25-50 \%$ & 70 & 52.2 & $32.63(9.3)$ & $26.14(7.4)$ & $36.70(4.6)$ & $9.67(1.6)$ \\
\hline Greater than $50 \%$ & 16 & 11.9 & $29.94(9.6)^{1}$ & $26.50(6.0)$ & $36.25(4.9)$ & $9.13(1.5)$ \\
\hline \multicolumn{7}{|l|}{ Personal History of LBP } \\
\hline No history of LBP & 5 & 3.7 & $40.40(11.8)$ & $28.40(10.6)$ & $36.20(3.4)$ & $8.00(4.4)$ \\
\hline History of LBP & 129 & 96.3 & $32.91(9.3)$ & $26.46(7.0)$ & $36.49(4.4)$ & $9.47(1.7)$ \\
\hline \multicolumn{7}{|l|}{$\begin{array}{l}\text { Impact of LBP on the } \\
\text { physical therapist's life } \\
(\mathrm{N}=129)\end{array}$} \\
\hline None & 40 & 31.0 & $29.57(6.9)$ & $24.38(6.5)$ & $37.72(4.3)$ & $8.43(3.8)$ \\
\hline Minimal & 76 & 58.9 & $33.89(9.2)$ & $27.22(7.1)$ & $36.08(4.4)$ & $9.38(1.5)$ \\
\hline Significant & 13 & 10.1 & $37.38(13.2)$ & $28.38(6.7)$ & $35.08(4.0)$ & $9.61(1.9)$ \\
\hline
\end{tabular}




\begin{tabular}{|l|l|l|l|l|l|l|}
\hline Exercise Frequency & & & & & & \\
\hline None & 4 & 3.0 & $35.50(2.4)$ & $30.00(1.8)$ & $34.00(2.1)$ & $8.00(1.6)$ \\
\hline 1 time a week & 7 & 5.2 & $30.29(9.8)$ & $28.14(6.4)$ & $36.57(5.2)$ & $9.78(1.7)$ \\
\hline 2 times a week & 23 & 17.2 & $33.91(12.5)$ & $27.30(7.7)$ & $36.70(4.0)$ & $9.93(1.7)$ \\
\hline 3 times a week & 30 & 22.4 & $32.67(9.3)$ & $26.77(6.3)$ & $37.13(3.8)$ & $9.23(1.9)^{1}$ \\
\hline 4 times a week & 30 & 22.4 & $33.93(11.0)$ & $25.50(7.6)$ & $36.70(5.7)$ & $9.23(1.9)$ \\
\hline 5 times a week & 23 & 17.2 & $31.65(7.1)$ & $25.35(7.8)$ & $35.43(4.5)$ & $8.57(2.0)$ \\
\hline More than 5 times & 17 & 12.7 & $34.53(10.1)$ & $27.00(7.2)$ & $36.59(3.1)$ & $9.88(1.3)^{1}$ \\
\hline Exercise Type & & & & & & \\
\hline Cardiovascular exercise & 114 & 81.4 & $33.17(9.3)$ & $26.34(7.0)$ & $36.47(4.5)$ & $9.43(1.6)$ \\
\hline $\begin{array}{l}\text { Machine based strength } \\
\text { training }\end{array}$ & 43 & 30.7 & $33.86(9.6)$ & $26.16(7.6)$ & $36.60(5.0)$ & $9.58(1.7)$ \\
\hline Free weight strength training & 72 & 51.4 & $32.85(8.9)$ & $25.47(7.2)^{1}$ & $36.96(4.5)$ & $9.56(1.7)$ \\
\hline $\begin{array}{l}\text { High Intensity Interval } \\
\text { training }\end{array}$ & 42 & 30.0 & $33.36(9.1)$ & $25.45(8.0)$ & $36.55(5.0)$ & $9.33(2.0)$ \\
\hline
\end{tabular}

Mean scores include standard deviation in parenthesis, Superscript numbers (1:1. 2:2...) indicate a significant difference ( $p \leq 0.05)$. HC-PAIRS-Health Care Providers' Pain and Impairment Relationship Scale, PABS-BM-Pain Attitudes and Beliefs Scale for Physiotherapists biomedical subscale, PABS-BPS-Pain Attitudes and Beliefs Scale for Physiotherapists biopsychosocial subscale, NPQ- Neurophysiology of Pain Questionnaire.

Table 5. Scores for beliefs and knowledge questionnaires.

\begin{tabular}{|l|l|l|l|l|}
\hline & HC-PAIRS & PABS-BM & PABS-BPS & NPQ \\
\hline Minimum & 18 & 12 & 27 & 2 \\
\hline Maximum & 64 & 45 & 48 & 12 \\
\hline Mean & 33.40 & 26.56 & 36.47 & 9.15 \\
\hline Median & 33.00 & 25.50 & 37.00 & 10 \\
\hline Mode & 36 & 25 & 34 & 10 \\
\hline
\end{tabular}

HC-PAIRS-Health Care Providers' Pain and Impairment Relationship Scale, PABS-BM-Pain Attitudes and Beliefs Scale for Physiotherapists biomedical subscale, PABS-BPS-Pain Attitudes and Beliefs Scale for Physiotherapists biopsychosocial subscale, NPQ- Neurophysiology of Pain Questionnaire.

\section{Simple Regression}

Simple regression was performed to predict HC-PAIRS and both subscales of the PABS-

PT based on physical therapist characteristics (Table 6). Entry-level degree, certification, setting, percentage of caseload made up of patients with LBP, and NPQ scores were all significant 
univariate predictors of HC-PAIRS scores. An entry-level Bachelor's degree $(B=4.190,95 \%$ $\left.\mathrm{Cl}[.532,7.848], \mathrm{R}^{2}=.036, \mathrm{p}=.025\right)$, no certifications $\left(\mathrm{B}=3.278,95 \% \mathrm{Cl}[.148,6.409], \mathrm{R}^{2}=.030, \mathrm{p}=\right.$ .040), working in orthopedic settings other than a private practice or hospital based setting $(B=$ $\left.4.403,95 \% \mathrm{Cl}[.342,8.465], \mathrm{R}^{2}=.032, \mathrm{p}=.034\right)$, and having a caseload made up of less than $10 \%$ patients with $\operatorname{LBP}\left(B=7.723,95 \% \mathrm{Cl}[1.036,14.411] \mathrm{R}^{2}=.036, p=.024\right)$ were predictive of higher HC-PAIRS scores. An entry-level Doctorate degree $\left(B=-3.280,95 \% \mathrm{CI}[-6.421,-.140] \mathrm{R}^{2}=.030\right.$, $p=.041)$, board certification $\left(B=-4.598,95 \% \mathrm{Cl}[-8.039,-1.157], R^{2}=.048, p=.009\right)$ and higher $N P Q$ scores $\left(B=-2.046,95 \% \mathrm{Cl}[-2.861,-1.232] \mathrm{R}^{2}=.156, \mathrm{p}=<.001\right)$ were predictive of lower HC-PAIRS scores.

Age, years of experience, entry-level degree, residency training, fellowship training, certifications, practice setting, and NPQ scores were predictors of PABS-BM scores. An age of 18-34 $\left(B=-2.992,95 \% \mathrm{Cl}[-5.417,-.567], R^{2}=.041, p=.016\right), 5-9$ years of experience $(B=-3.259$, 95\% Cl[-6.294,-.224], $\left.\mathrm{R}^{2}=.032, \mathrm{p}=.036\right)$, an entry-level Doctorate degree $(\mathrm{B}=-3.311,95 \% \mathrm{Cl}[-$ 5.628,-.995], $\left.R^{2}=.055, p=.005\right)$, residency training $\left(B=-3.561,95 \% C l[-6.994,-.128], R^{2}=.031\right.$, $p=.042)$, fellowship training $\left(B=-5.433,95 \% \mathrm{Cl}[-9.589,-1.277], R^{2}=.048, p=.011\right)$, board certification $\left(B=-4.469,95 \% C l[-6.994,-1.943], R^{2}=.081, p=<.001\right)$, and higher NPQ scores $(B=-$ $\left.1.943,95 \% \mathrm{Cl}[-2.524,-1.362], \mathrm{R}^{2}=.246, \mathrm{p}=<.001\right)$ were all predictive of lower scores on the PABS-BM. An entry-level Bachelor's degree ( $\left.B=2.760,95 \% \mathrm{Cl}[.016,5.504], R^{2}=.028, p=.049\right)$, no certifications $\left(B=3.293,95 \% \mathrm{Cl}[.984,5.602], \mathrm{R}^{2}=.054, \mathrm{p}=.006\right)$, and practicing in a setting other than a private orthopedic clinic or hospital based orthopedic clinic $(B=3.802,95 \%$ $\left.\mathrm{CI}[.785,6.819], \mathrm{R}^{2}=.043, \mathrm{p}=.014\right)$ were associated with higher PABS-BM scores. 
Age, years of experience, entry-level degree, residency training, fellowship training, certifications, and NPQ scores were predictive of PABS-BPS scores. Age 18-34 ( $B=2.477,95 \%$ $\left.\mathrm{Cl}[.981,3.974], \mathrm{R}^{2}=.072, \mathrm{p}=.001\right)$, residency training $\left(\mathrm{B}=2.878,95 \% \mathrm{Cl}[.789,4.967], \mathrm{R}^{2}=.053\right.$, $p=.007)$, fellowship training $\left(B=2.587,95 \% \mathrm{Cl}[.003,5.172], \mathrm{R}^{2}=.029, p=.050\right)$, board certification $\left(B=3.007,95 \% C l[1.434,4.581], R^{2}=.094, p<.001\right)$, and higher $N P Q$ scores $(B=.895,95 \%$ $\left.\mathrm{Cl}[.505,1.285], \mathrm{R}^{2}=.133, \mathrm{p}<.001\right)$ were associated with higher PABS-PBS scores. Age over 55 ( $\mathrm{B}=-$ $\left.2.516,95 \% \mathrm{Cl}[-4.373,-.658], \mathrm{R}^{2}=.049, \mathrm{p}=.008\right)$, over 25 years of experience $(\mathrm{B}=-2.392,95 \% \mathrm{Cl}[-$ 4.039,-.745], $\left.R^{2}=.056, p=.005\right)$, an entry-level Bachelor's degree $(B=-2.697,95 \% C l[-4.365,-$ $\left..992], R^{2}=.067, p=.002\right)$, and no certifications $\left(B=-2.346,95 \% C l[-3.782,-.910], R^{2}=.070, p=.002\right)$ were associated with lower PABS-BPS scores.

Table 6. Simple linear regression of beliefs scores using physical therapist demographics as predictors.

\begin{tabular}{|c|c|c|c|c|c|c|c|c|c|c|c|c|}
\hline & \multicolumn{12}{|c|}{ Simple Linear Regression } \\
\hline & \multicolumn{4}{|c|}{ HC-PAIRS } & \multicolumn{4}{|c|}{ PABS-BM } & \multicolumn{4}{|c|}{ PABS-BPS } \\
\hline & $B$ & $F$ & $p$ & $R^{2}$ & $B$ & $F$ & $p$ & $R^{2}$ & $B$ & $F$ & $p$ & $R^{2}$ \\
\hline $\begin{array}{l}18-34 \text { Years } \\
\text { Old }\end{array}$ & -3.084 & 3.502 & .063 & .025 & -2.992 & 5.954 & .016 & .041 & 2.477 & 10.714 & .001 & .072 \\
\hline $\begin{array}{l}35-44 \text { Years } \\
\text { Old }\end{array}$ & 2.211 & 1.435 & .233 & .010 & .817 & .601 & .439 & .004 & .016 & .020 & .889 & .000 \\
\hline $\begin{array}{l}45-54 \text { Years } \\
\text { Old }\end{array}$ & -1.902 & .911 & .342 & .007 & .777 & .539 & .464 & .004 & -1.125 & 1.843 & .177 & .013 \\
\hline $\begin{array}{l}>55 \text { Years } \\
\text { Old }\end{array}$ & 3.661 & 3.711 & .056 & .026 & 2.575 & 2.868 & .093 & .020 & -2.516 & 7.169 & .008 & .049 \\
\hline Male & -3.092 & 3.865 & .051 & .027 & -1.594 & 1.799 & .182 & .006 & .618 & .682 & .410 & .005 \\
\hline Female & 2.112 & 2.480 & .118 & .018 & 1.125 & .898 & .345 & .006 & -.592 & .613 & .435 & .004 \\
\hline $\begin{array}{l}\leq 4 \text { Yrs. } \\
\text { Experience }\end{array}$ & 1.091 & 1.008 & .317 & .007 & .436 & .268 & .605 & .002 & 1.267 & 3.027 & .084 & .021 \\
\hline
\end{tabular}




\begin{tabular}{|c|c|c|c|c|c|c|c|c|c|c|c|c|}
\hline $\begin{array}{l}5-9 \text { Yrs. } \\
\text { Experience }\end{array}$ & -3.680 & 3.178 & .077 & .023 & -3.259 & 4.509 & .036 & .032 & 1.423 & 2.147 & .145 & .015 \\
\hline $\begin{array}{l}10-14 \text { Yrs. of } \\
\text { Experience }\end{array}$ & 2.193 & 2.191 & .141 & .016 & -1.493 & 1.121 & .292 & .008 & -1.301 & 2.052 & .154 & .015 \\
\hline $\begin{array}{l}\text { 15-19 Yrs. of } \\
\text { Experience }\end{array}$ & -.459 & .143 & .707 & .001 & .123 & .149 & .700 & .001 & 1.339 & 3.187 & .076 & .023 \\
\hline $\begin{array}{l}\text { 20-24 Yrs. of } \\
\text { Experience }\end{array}$ & 2.221 & .973 & .326 & .007 & 2.245 & 1.523 & .219 & .011 & .066 & .003 & .954 & .000 \\
\hline $\begin{array}{l}\geq 25 \text { Yrs. of } \\
\text { Experience }\end{array}$ & -.761 & .176 & .676 & .001 & 1.232 & 1.210 & .273 & .009 & -2.392 & 8.249 & .005 & .056 \\
\hline Bachelor's & 4.109 & 5.130 & .025 & .036 & 2.760 & 3.956 & .049 & .028 & -2.679 & 9.863 & .002 & .067 \\
\hline Master's & -2.523 & 1.963 & .163 & .014 & .088 & .139 & .710 & .001 & .976 & 1.331 & .251 & .010 \\
\hline Doctorate & -3.280 & 4.265 & .041 & .030 & -3.311 & 7.993 & .005 & .055 & 1.372 & 3.379 & .068 & .024 \\
\hline $\begin{array}{l}\text { No Post } \\
\text { Graduate } \\
\text { Education }\end{array}$ & -.275 & .029 & .865 & .000 & 1.792 & 2.239 & .137 & .016 & -1.392 & 3.463 & .065 & .024 \\
\hline tDPT & -.524 & .215 & .644 & .007 & -1.371 & .638 & .426 & .005 & .602 & .276 & .600 & .002 \\
\hline Residency & -3.868 & 2.80 & .097 & .020 & -3.561 & 4.221 & .042 & .031 & 2.878 & 7.426 & .007 & .053 \\
\hline Fellowship & -4.477 & 2.50 & .116 & .018 & -5.433 & 6.686 & .011 & .048 & 2.587 & 3.921 & .050 & .029 \\
\hline $\begin{array}{l}\text { Board } \\
\text { Certification }\end{array}$ & -4.598 & 6.980 & .009 & .048 & -4.469 & 12.243 & $<.001$ & .081 & 3.007 & 14.284 & $<.001$ & .094 \\
\hline $\begin{array}{l}\text { Manual } \\
\text { Therapy } \\
\text { Certification }\end{array}$ & -.151 & .271 & .603 & .002 & .543 & .136 & .713 & .001 & -.167 & .226 & .635 & .002 \\
\hline $\begin{array}{l}\text { Board and } \\
\text { Manual } \\
\text { Therapy } \\
\text { Certification }\end{array}$ & -2.844 & .437 & .510 & .003 & -4.726 & 2.190 & .141 & .016 & 1.70 & .337 & .563 & .002 \\
\hline $\begin{array}{l}\text { No } \\
\text { Certification }\end{array}$ & 3.278 & 6.652 & .040 & .030 & 3.293 & 7.954 & .006 & .054 & -2.346 & 10.437 & .002 & .070 \\
\hline Private Ortho & -1.068 & 2.195 & .141 & .016 & -2.263 & 3.474 & .064 & .025 & .823 & 1.645 & .202 & .012 \\
\hline $\begin{array}{l}\text { Hospital } \\
\text { Ortho }\end{array}$ & .513 & .300 & .585 & .002 & 1.514 & 1.384 & .241 & .010 & -1.491 & 3.468 & .065 & .026 \\
\hline $\begin{array}{l}\text { Other Ortho } \\
\text { Settings }\end{array}$ & 4.403 & 4.596 & .034 & .032 & 3.802 & 6.208 & .014 & .043 & 1.00 & .508 & .477 & .004 \\
\hline $\begin{array}{l}\text { LBP Case } \\
\text { Load }<10 \%\end{array}$ & 7.723 & 5.215 & .024 & .036 & 4.182 & 2.692 & .103 & .019 & -1.428 & .787 & .377 & .006 \\
\hline $\begin{array}{l}\text { LBP Case } \\
\text { Load } 10-24 \%\end{array}$ & .885 & .250 & .618 & .002 & -.185 & .020 & .889 & .000 & -.030 & .001 & .971 & .000 \\
\hline $\begin{array}{l}\text { LBP Case } \\
\text { Load } 25-50 \%\end{array}$ & -1.429 & .802 & .372 & .006 & -.829 & .483 & .488 & .003 & .457 & .373 & .542 & .003 \\
\hline $\begin{array}{l}\text { LBP Case } \\
\text { Load }>50 \%\end{array}$ & -3.845 & 2.380 & .125 & .017 & -.065 & .001 & .973 & .000 & -.250 & .045 & .832 & .000 \\
\hline
\end{tabular}




\begin{tabular}{|c|c|c|c|c|c|c|c|c|c|c|c|c|}
\hline $\begin{array}{l}\text { No Beliefs } \\
\text { Change }\end{array}$ & .991 & 1.126 & .290 & .008 & .944 & .540 & .464 & .004 & -.953 & 2.044 & .155 & .015 \\
\hline $\begin{array}{l}\text { Minimal } \\
\text { Beliefs } \\
\text { Change }\end{array}$ & -.706 & .194 & .660 & .001 & -1.341 & 1.267 & .262 & .009 & .828 & 1.271 & .261 & .009 \\
\hline $\begin{array}{l}\text { Sig. Beliefs } \\
\text { Change }\end{array}$ & -1.602 & .609 & .436 & .004 & .591 & .373 & .542 & .001 & .460 & .247 & .620 & .002 \\
\hline $\begin{array}{l}\text { LBP Minimal } \\
\text { Impact }\end{array}$ & 1.207 & 1.513 & .221 & .011 & 1.458 & 1.760 & .187 & .031 & -.859 & 1.969 & .163 & .014 \\
\hline $\begin{array}{l}\text { LBP } \\
\text { Significant } \\
\text { Impact }\end{array}$ & 4.455 & 2.665 & .105 & .019 & 2.015 & 1.123 & .291 & .008 & -1.537 & 1.432 & .233 & .001 \\
\hline $\begin{array}{l}\text { Exercise } \\
1 \mathrm{x} / \text { week or } \\
\text { Less }\end{array}$ & -1.260 & .180 & .672 & .001 & 2.454 & 1.232 & .269 & .009 & -.906 & .425 & .516 & .003 \\
\hline $\begin{array}{l}\text { Exercise } \\
2 x / \text { week }\end{array}$ & .682 & .100 & .752 & .001 & .894 & .308 & .580 & .002 & .268 & .070 & .791 & .001 \\
\hline $\begin{array}{l}\text { Exercise } \\
3 x / \text { week }\end{array}$ & -.861 & .195 & .659 & .001 & .267 & .034 & .855 & .000 & .842 & .856 & .356 & .006 \\
\hline $\begin{array}{l}\text { Exercise } \\
4 x / \text { week }\end{array}$ & .752 & .149 & .700 & .001 & -1.345 & .859 & .356 & .006 & .291 & .102 & .750 & .001 \\
\hline $\begin{array}{l}\text { Exercise } \\
5 x / \text { week }\end{array}$ & -.655 & .137 & .712 & .001 & -.710 & .289 & .592 & .002 & -.765 & .856 & .357 & .006 \\
\hline $\begin{array}{l}\text { Cardiovascul } \\
\text { ar Exercise }\end{array}$ & -.949 & .260 & .645 & .002 & -1.158 & .570 & .451 & .004 & .012 & .000 & .990 & .000 \\
\hline $\begin{array}{l}\text { Machine- } \\
\text { Based } \\
\text { Strengthen } \\
\text { Exercise }\end{array}$ & .747 & .186 & .667 & .001 & -.569 & .193 & .661 & .001 & .192 & .056 & .813 & .000 \\
\hline $\begin{array}{l}\text { Free Weight } \\
\text { Based } \\
\text { Strengthen } \\
\text { Exercise }\end{array}$ & -1.020 & .408 & .524 & .003 & -2.234 & 3.583 & .025 & .025 & 1.002 & 1.811 & .181 & .013 \\
\hline $\begin{array}{l}\text { High } \\
\text { Intensity } \\
\text { Training }\end{array}$ & .248 & .165 & .686 & .001 & -1.569 & 2.11 & .149 & .015 & .102 & .114 & .736 & .001 \\
\hline NPQ & -2.046 & 24.697 & $<.001$ & .156 & -1.943 & 43.804 & $<.001$ & .246 & .895 & 20.568 & $<.001$ & .133 \\
\hline
\end{tabular}

HC-PAIRS-Health Care Providers' Pain and Impairment Relationship Scale, PABS-BM-Pain Attitudes and Beliefs Scale for

Physiotherapists biomedical subscale, PABS-BPS-Pain Attitudes and Beliefs Scale for Physiotherapists biopsychosocial subscale, NPQ- Neurophysiology of Pain Questionnaire.

Multiple Linear Regression

HC-PAIRS

The seven significant predictors from the simple regression were entered into a best

subsets regression. One hundred twenty-seven models were created and sorted by AIC. The 
model with the lowest AIC (585.868) excluded three predictors, an entry-level Doctoral degree, board certification, and no certifications. Backwards multiple regression was performed with the remaining 4 variables, which included an entry-level Bachelor's degree, working in orthopedic settings other than a private practice or hospital-based setting, having a caseload made up of less than $10 \%$ patients with LBP, and NPQ scores (Table 7). Variance inflation factor (VIF) scores were below 2.0 and Pearson Correlations were less than .190, which indicated that multicollinearity was not present. A model containing all 6 of the predictors significantly predicted HC-PAIRS scores $(p<.001)$ with an adjusted $R^{2}$ of .189 . NPQ score was the only significant coefficient in this model. After the removal of the caseload variable, the adjusted $\mathrm{R}^{2}$ dropped to .176 and both NPQ scores $(p<.001)$ and the practice-setting variable $(p=.050)$ were significant predictors. The entry-level Bachelor's degree variable was then removed, leaving a two variable model that was significant $(p<.001)$ and had an adjusted $R^{2}$ of .162 . In this model, the NPQ coefficient had a $p$-value of $<.001$ and the practice setting variable had a $p$-value of .069. This left NPQ as the only significant predictor of HC-PAIRS with an adjusted $R^{2}$ of .147 and a $\beta$ of $-2.31,95 \% \mathrm{Cl}[-2.851,-1.213]$.

Table 7. Multiple regression modeling to predict HC-PAIRS scores.

\begin{tabular}{|c|c|c|c|c|c|c|}
\hline & \multicolumn{6}{|c|}{ HC-PAIRS Multiple Regression } \\
\hline & \multirow[t]{2}{*}{ Coefficients } & \multirow[t]{2}{*}{$\beta$} & \multirow{2}{*}{$\begin{array}{c}\text { Standardized } \\
\beta\end{array}$} & \multicolumn{2}{|c|}{$95 \% \mathrm{Cl}$} & \multirow[t]{2}{*}{$P$} \\
\hline & & & & Lower Bound & Upper Bound & \\
\hline \multirow{4}{*}{$\begin{array}{l}\text { Model } 1 \\
C=47.574 \\
R^{2}=.213 \\
A^{2}=.189 \\
p<.001\end{array}$} & NPQ & -1.713 & -.330 & -2.538 & -.888 & $<.001$ \\
\hline & Setting other than & 3.429 & .142 & -.352 & 7.211 & .075 \\
\hline & $\begin{array}{l}\text { Entry-level Bachelor's } \\
\text { Degree }\end{array}$ & 3.225 & .147 & -.209 & 6.659 & .065 \\
\hline & $\begin{array}{l}\text { Case load of less than } \\
10 \% \text { LBP }\end{array}$ & 5.521 & .139 & -.691 & 11.734 & .081 \\
\hline & NPQ & -1.796 & -346 & -2.622 & -.970 & $<.001$ \\
\hline
\end{tabular}




\begin{tabular}{|c|c|c|c|c|c|c|}
\hline Model 2 & Setting other than & 3.793 & .157 & .004 & 7.582 & .050 \\
\hline $\begin{array}{l}C=48.625 \\
R^{2}=.195 \\
A^{2}=.176 \\
p<.001\end{array}$ & $\begin{array}{l}\text { Entry-level Bachelor's } \\
\text { Degree }\end{array}$ & 3.186 & .146 & -.273 & 7.350 & .069 \\
\hline $\begin{array}{l}\text { Model } 3 \\
C=50.828\end{array}$ & NPQ & -1.943 & -.374 & -2.760 & -1.125 & $<.001$ \\
\hline $\begin{array}{l}\mathrm{R}^{2}=.174 \\
\mathrm{AR}^{2}=.162 \\
\mathrm{p}<.001\end{array}$ & Setting other than & 3.539 & .146 & -.273 & 7.350 & .069 \\
\hline $\begin{array}{l}\text { Model } 4 \\
C=52.325 \\
R^{2}=.153 \\
A^{2}=.147 \\
p<.001\end{array}$ & NPQ & -2.032 & -.392 & -2.851 & -1.213 & $<.001$ \\
\hline
\end{tabular}

HC-PAIRS-Health Care Providers' Pain and Impairment Relationship Scale, C-Constant, AR ${ }^{2}$-Adjusted R²

\section{PABS-BM}

The 10 significant predictors from the simple regression were entered into a best

subsets regression. One thousand twenty-three models were created and sorted by AIC. The model with the lowest AIC (483.372) excluded five predictors: 5-9 years of experience, an entrylevel Bachelor's degree, an entry-level Doctorate degree, residency training, and no certifications. Backwards multiple regression was performed with the remaining five variables, which included working in orthopedic settings other than a private practice or hospital-based setting, an age of 18-34, fellowship training, board certification, and NPQ scores (Table 8). VIF was below 2.0 and Pearson Correlations were less than .243, which indicated that

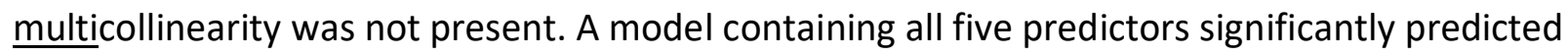
PABS-BM scores $(p<.001)$ with an adjusted $R^{2}$ of .298 . An age of $18-34(p=.024)$ and NPQ $(p<.001)$ were the only significant coefficients in the model. After removal of fellowship training, the adjusted $R^{2}$ dropped to .291 and an age of $18-34(p=.027)$, board certification $(p=.032)$, and NPQ scores $(p<.001)$ were significant predictors. The final model significantly predicted PABS-BM scores $(p<.001)$ with an $R^{2}$ of .278 . The model included an age of $18-34(\beta=-$ 
2.275, 95\% Cl [-4.442,-.108], $p=.040)$ board certification $(\beta=-3.030,95 \% \mathrm{Cl}[-5.374,-.686]$,

$p=.012)$, and NPQ scores $(\beta=-1.63,95 \% \mathrm{CI}[-2.232,-1.047], p<.001)$.

Table 8. Multiple regression modeling to predict PABS-BM scores.

\begin{tabular}{|c|c|c|c|c|c|c|}
\hline & \multicolumn{6}{|c|}{ PABS-BM Multiple Regression } \\
\hline & \multirow[t]{2}{*}{ Coefficients } & \multirow[t]{2}{*}{$\beta$} & \multirow{2}{*}{$\begin{array}{c}\text { Standardized } \\
\beta\end{array}$} & \multicolumn{2}{|c|}{$95 \% \mathrm{Cl}$} & \multirow[t]{2}{*}{$P$} \\
\hline & & & & Lower Bound & Upper Bound & \\
\hline \multirow{5}{*}{$\begin{array}{l}\text { Model } 1 \\
C=42.351 \\
\mathrm{R}^{2}=.325 \\
\mathrm{AR}^{2}=.298 \\
\mathrm{p}<.001\end{array}$} & $\begin{array}{l}\text { Ortho setting other than } \\
\text { private practice or hospital }\end{array}$ & 2.464 & -.330 & -.225 & 5.154 & .072 \\
\hline & Age between 18 and 34 & -2.484 & -.169 & -4.628 & -.340 & .024 \\
\hline & Fellowship Training & -2.816 & -.114 & -6.482 & .849 & .131 \\
\hline & Board Certification & -2.313 & -.149 & -4.699 & .072 & .057 \\
\hline & $\mathrm{NPQ}$ & -1.534 & -.394 & -2.125 & -.943 & $<.001$ \\
\hline \multirow{4}{*}{$\begin{array}{l}\text { Model } 2 \\
\mathrm{C}=42.715 \\
\mathrm{R}^{2}=.313 \\
\mathrm{AR}^{2}=.291 \\
\mathrm{p}<.001\end{array}$} & $\begin{array}{l}\text { Ortho setting other than } \\
\text { private practice or hospital }\end{array}$ & 2.523 & .139 & -.179 & 5.225 & .067 \\
\hline & Age between 18 and 34 & -2.432 & -.166 & -4.586 & -.278 & .027 \\
\hline & Board Certification & -2.599 & -.167 & -4.967 & -.231 & .032 \\
\hline & NPQ & -1.594 & -.409 & -2.183 & -1.005 & $<.001$ \\
\hline \multirow{3}{*}{$\begin{array}{l}\text { Model } 3 \\
C=43.681 \\
R^{2}=.294 \\
A R^{2}=.278 \\
p<.001\end{array}$} & Age between 18 and 34 & -2.275 & -.155 & -4.442 & -.108 & .040 \\
\hline & Board Certification & -3.030 & -.195 & -5.374 & -.686 & .012 \\
\hline & NPQ & -1.639 & -.421 & -2.232 & -1.047 & $<.001$ \\
\hline
\end{tabular}

PABS-BM-Pain Attitudes and Beliefs Scale for Physiotherapists biomedical subscale, C-Constant, AR ${ }^{2}$-Adjusted R ${ }^{2}$

\section{PABS-BPS}

The nine significant predictors from the simple regression were entered into a best

subsets regression. Five hundred eleven models were created and sorted by AIC. The model with the lowest AIC (363.939) excluded six predictors: an age over 55, over 25 years of experience, an entry-level Bachelor's degree, residency training, fellowship training, and no certification. Backwards multiple regression was performed with the remaining three variables (Table 9). VIF was below 2.0 and Pearson Correlations were less than .245, which indicated that multicollinearity was not present. A model containing all three predictors significantly predicted 
PABS-PBS scores $(p<.001)$ with an adjusted $R^{2}$ of .233. The model included an age of 18-34

$(\beta=2.320,95 \% \mathrm{Cl}[.949,3.690], p=.001)$, board certification $(\beta=2.577,95 \% \mathrm{Cl}[1.093,4.061], p=$ .001 , and $N P Q$ scores $(\beta=.612,95 \% \mathrm{Cl}[.237, .987], p=.002)$.

Table 9. Multiple regression modeling to predict PABS-BPS scores.

\begin{tabular}{|c|c|c|c|c|c|c|}
\hline & \multicolumn{6}{|c|}{ PABS-BPS Multiple Regression } \\
\hline & \multirow[t]{2}{*}{ Coefficients } & \multirow[t]{2}{*}{$\beta$} & \multirow{2}{*}{$\begin{array}{c}\text { Standardized } \\
\beta\end{array}$} & \multicolumn{2}{|c|}{$95 \% \mathrm{Cl}$} & \multirow[t]{2}{*}{$P$} \\
\hline & & & & Lower Bound & Upper Bound & \\
\hline Model 1 & Age between 18 and 34 & 2.320 & .257 & .949 & 3.690 & .001 \\
\hline $\begin{array}{l}C=29.110 \\
R^{2}=.250\end{array}$ & Board Certification & 2.577 & .269 & 1.093 & 4.061 & .001 \\
\hline $\begin{array}{l}\mathrm{AR}^{2}=.233 \\
\mathrm{p}<.001\end{array}$ & NPQ & .612 & .255 & .237 & .987 & .002 \\
\hline
\end{tabular}

PABS-BPS-Pain Attitudes and Beliefs Scale for Physiotherapists biopsychosocial subscale, C-Constant, AR ${ }^{2}$-Adjusted R ${ }^{2}$

\section{Patient Data Findings}

FOTO was able to identify 105 of the 140 (75\%) physical therapists who responded to the survey. These therapists had completed cases for 3,201 patients with LBP. Outlier analysis identified four physical therapists with extreme values for the number of completed cases. One therapist had 268 completed cases and three therapists had 155 completed cases in 2018 . This was more than 2.2 times the interquartile range and were removed. Nine therapists had less than five completed cases and were not included in the analysis. No other outliers were present in the data.

Descriptive statistics for patient data are reported in table 10. Average patient values for a 5 patient cut-off sample, 10 patient cut-off sample, and 20 patient cut-off sample were based on data from $2,448,2,345$, and 2,016 patients respectively. The mean of the average patient age ranged from 57.3 years for a 10 patient cut-off to 56.2 years for a 5 patient cut-off. Patient 
age ranged from a low of less than 1 to high of 89 years. The mean average intake

Computerized Lumbar Functional Status measure (CLFS) was very similar for the three cut-off points and ranged from 48.6 to 48.9 . The mean average CLFS change score was 15.7 and the mean average residual score was 1.6 for all three cut-off points. Mean average Fear Avoidance Belief Scale Physical Activity subscale (FABQpa) change ranged from -6.1 to -6.8 points across samples. Physical therapist beliefs scores and NPQ varied by less than 1 point across samples. HC-PAIRS scores ranged from 31.0 to 31.8 , PABS-BM scores ranged from 25.4 to 25.88 , PABSBSP ranged from 36.48 to 37.0 , and NPQ scores ranged from 9.4 to 9.42 across samples.

Table 10. Patient data, physical therapist beliefs, and knowledge by sample.

\begin{tabular}{|c|c|c|c|c|c|c|}
\hline \multirow{2}{*}{$\begin{array}{l}\text { Number of patients } \\
\text { per therapist cut } \\
\text { off }\end{array}$} & \multicolumn{2}{|c|}{$\begin{array}{l}\geq 5 \text { patients } \\
n=92 \text { physical therapists } \\
2,448 \text { patients }\end{array}$} & \multicolumn{2}{|c|}{$\begin{array}{l}\geq 10 \text { patients } \\
n=78 \text { physical therapists } \\
2,345 \text { patients }\end{array}$} & \multicolumn{2}{|c|}{$\begin{array}{l}\geq 20 \text { patients } \\
n=54 \text { physical therapists } \\
2,016 \text { patients }\end{array}$} \\
\hline & Mean & Min/Max & Mean & Min/Max & Mean & Min/Max \\
\hline Number of Patients & $26.6(18.7)$ & $5 / 89$ & $30.1(18.2)$ & $10 / 89$ & $37.3(17.4)$ & $20 / 89$ \\
\hline $\begin{array}{l}\text { Average Patient Age } \\
\text { (Yrs) }\end{array}$ & $56.2(7.5)$ & $41 / 76$ & $57.3(6.7)$ & $43 / 76$ & $57.2(5.5)$ & $43 / 70$ \\
\hline Average Intake CLFS & $48.9(4.9)$ & $27.5 / 58.3$ & $48.6(5.0)$ & $27.5 / 58.3$ & $48.9(5.0)$ & $27.5 / 57.9$ \\
\hline $\begin{array}{l}\text { Average CLFS } \\
\text { Change }\end{array}$ & $15.7(5.8)$ & $3.7 / 34.7$ & $15.7(5.0)$ & $7.5 / 32.2$ & $15.7(4.7)$ & $7.5 / 28.1$ \\
\hline $\begin{array}{l}\text { Average CLFS } \\
\text { Residual Score }\end{array}$ & $1.6(4.8)$ & $-10 \cdot 9 / 15.2$ & $1.6(4.4)$ & $-6.2 / 15.2$ & $1.6(3.7)$ & $-6.2 / 13.3$ \\
\hline $\begin{array}{l}\text { Average Intake } \\
\text { FABQpa }\end{array}$ & $47.7(9.3)$ & $26 / 100$ & $47.5(9.1)$ & $34.2 / 100$ & $47.4(8.6)$ & $35.5 / 100$ \\
\hline $\begin{array}{l}\text { Average FABQpa } \\
\text { Change }\end{array}$ & $-6.1(9.6)$ & $-41.5 / 21$ & $-6.9(9.4)$ & $-41.5 / 16.0$ & $-6.8(7.3)$ & $-26.5 / 7.2$ \\
\hline $\begin{array}{l}\text { Average Number of } \\
\text { Visits }\end{array}$ & $10.0(3.5)$ & $4 / 23$ & $10.3(3.3)$ & $5 / 23$ & $9.7(3.0)$ & $5 / 17$ \\
\hline PT HC-PAIRS Score & $31.82(8.2)$ & $18 / 56$ & $31.0(8.0)$ & $18 / 53$ & $31.7(7.9)$ & $18 / 53$ \\
\hline PT PABS-BM Score & $25.88(6.8)$ & $13 / 45$ & $25.6(6.8)$ & $13 / 45$ & $25.4(6.236)$ & $15 / 45$ \\
\hline $\begin{array}{l}\text { PT PABS-BSP } \\
\text { Scores }\end{array}$ & $36.48(4.6)$ & $27 / 48$ & $37.0(4.5)$ & $27 / 48$ & $36.7(4.4)$ & $27 / 44$ \\
\hline PT NPQ Scores & $9.42(2.2)$ & $6 / 12$ & $9.4(2.3)$ & $6 / 12$ & $9.4(2.2)$ & $6 / 12$ \\
\hline
\end{tabular}




\begin{tabular}{|l|l|l|l|l|l|l|}
\hline $\begin{array}{l}\text { \% of Care Provided } \\
\text { by Other PT } \\
\text { Providers }\end{array}$ & $29.7(28.5)$ & $0 / 90$ & $29.5(29.0)$ & $0 / 90$ & $30.87(28.7)$ & $0 / 90$ \\
\hline
\end{tabular}

CLFS- Computerized Lumbar Functional Scale, FABQpa- Fear Avoidance Beliefs Questionnaire physical activity subscale, HCPAIRS-Health Care Providers' Pain and Impairment Relationship Scale, PABS-BM-Pain Attitudes and Beliefs Scale for Physiotherapists biomedical subscale, PABS-BPS-Pain Attitudes and Beliefs Scale for Physiotherapists biopsychosocial subscale, NPQ- Neurophysiology of Pain Questionnaire.

\section{Simple Linear Regression}

\section{Average CLFS Change}

Simple linear regression of CLFS change scores for the three cut-off samples are reported in Table 11. The three measures of beliefs were not significant predictors of CLFS change at the 5-patient or 20-patient cut-off, with p values ranging from .065 (HC-PAIRS) to .762 (PABS-BPS). Average FABQpa change $\left(B=-.183,95 \% \mathrm{Cl}[-.303,-.062], \mathrm{R}^{2}=.095, \mathrm{p}=.003\right)$ was the only significant predictor of CLFS change for the 5-patient cut-off sample. HC-PAIRS (B=.206, $\left.95 \% \mathrm{Cl}[.069, .343], R^{2}=.105, p=.004\right), \operatorname{PABS}-\mathrm{BM}\left(B=.187,95 \% \mathrm{Cl}[.024, .350], \mathrm{R}^{2}=.064, p=.025\right)$, and average $F A B Q p a$ change $\left(B=-.171,95 \% \mathrm{CI}[-.092, .163], \mathrm{R}^{2}=.100, \mathrm{p}=.005\right)$ were significant predictors of CLFS change in the 10-patient cut-off sample. Average intake FABQpa $(B=.147$, $\left.95 \% \mathrm{Cl}[-.232,-.030], \mathrm{R}^{2}=.074, \mathrm{p}=.049\right)$ and average FABQpa change $(\mathrm{B}=-.290,95 \% \mathrm{Cl}[-.451,-$ $\left..130], R^{2}=.206, p=.001\right)$ were the only two significant predictors of CLFS change for the 20patient cut-off sample.

Table 11. Simple linear regression of average CLFS change scores.

\begin{tabular}{|c|c|c|c|c|c|c|c|c|c|c|c|c|}
\hline & \multicolumn{12}{|c|}{$\begin{array}{l}\text { Simple Linear Regression } \\
\text { Average Computerized Lumbar Functional Status Change }\end{array}$} \\
\hline & \multicolumn{4}{|c|}{5 Patient Cut-off } & \multicolumn{4}{|c|}{10 Patient Cut-off } & \multicolumn{4}{|c|}{20 Patient Cut-off } \\
\hline & $B$ & $F$ & $p$ & $R^{2}$ & $B$ & $F$ & $p$ & $R^{2}$ & $B$ & $F$ & $p$ & $R^{2}$ \\
\hline HC-PAIRS & .116 & 2.477 & .119 & .027 & .206 & 8.939 & .004 & .105 & .150 & 3.554 & .065 & .064 \\
\hline PABS-BM & .134 & 2.319 & .131 & .025 & .187 & 5.201 & .025 & .064 & .107 & 1.072 & .305 & .020 \\
\hline PABS-BPS & .095 & .518 & .473 & .006 & -.109 & .725 & .397 & .009 & -.045 & .092 & .762 & .002 \\
\hline NPQ & -.031 & .012 & .912 & .000 & -.244 & .943 & .335 & .012 & .125 & .176 & .677 & .003 \\
\hline
\end{tabular}




\begin{tabular}{|c|c|c|c|c|c|c|c|c|c|c|c|c|}
\hline $\begin{array}{l}\text { Number of } \\
\text { Patients }\end{array}$ & -.023 & .491 & .485 & .005 & -.026 & .654 & .421 & .009 & -.040 & 1.161 & .286 & .022 \\
\hline $\begin{array}{l}\text { Average Intake } \\
\text { FABQpa }\end{array}$ & .076 & 1.333 & .252 & .015 & .035 & .303 & .584 & .004 & .147 & 4.052 & .049 & .074 \\
\hline $\begin{array}{l}\text { Average } \\
\text { FABQpa } \\
\text { Change }\end{array}$ & -.183 & 9.071 & .003 & .095 & -.171 & 8.333 & .005 & .100 & -.290 & 13.209 & .001 & .206 \\
\hline $\begin{array}{l}\text { Average } \\
\text { Number of } \\
\text { Visits }\end{array}$ & .143 & .658 & .419 & .007 & .134 & .584 & .447 & .008 & -.172 & .624 & .433 & .012 \\
\hline $\begin{array}{l}\leq 4 \text { Yrs. } \\
\text { Experience }\end{array}$ & -.095 & .004 & .949 & .000 & -1.515 & 1.127 & .292 & .015 & -.538 & .124 & .726 & .002 \\
\hline $\begin{array}{l}\text { 5-9 Yrs. } \\
\text { Experience }\end{array}$ & 1.477 & 1.061 & .306 & .012 & .605 & .210 & .648 & .003 & .158 & .009 & .923 & .000 \\
\hline $\begin{array}{l}\text { 10-14 Yrs. of } \\
\text { Experience }\end{array}$ & -1.034 & .233 & .630 & .003 & .446 & .044 & .834 & .001 & 1.110 & .259 & .613 & .005 \\
\hline $\begin{array}{l}\text { 15-19 Yrs. of } \\
\text { Experience }\end{array}$ & 1.897 & .879 & .351 & .010 & 1.555 & .701 & .405 & .009 & 2.476 & 1.553 & .218 & .030 \\
\hline $\begin{array}{l}20-24 \text { Yrs. of } \\
\text { Experience }\end{array}$ & 2.164 & 1.260 & .265 & .014 & 2.071 & 1.392 & .242 & .018 & -.011 & .000 & .996 & .000 \\
\hline $\begin{array}{l}\geq 25 \text { Yrs. of } \\
\text { Experience }\end{array}$ & -2.266 & 2.623 & .109 & .029 & -1.432 & 1.192 & .279 & .016 & -1.401 & .951 & .334 & .018 \\
\hline $\begin{array}{l}\text { 18-34 Years } \\
\text { Old }\end{array}$ & 1.177 & .910 & .343 & .010 & -.352 & .092 & .763 & .001 & -.029 & .000 & .983 & .000 \\
\hline $\begin{array}{l}\text { 35-44 Years } \\
\text { Old }\end{array}$ & -.249 & .028 & .868 & .000 & .168 & .014 & .905 & .000 & .482 & .106 & .746 & .002 \\
\hline $\begin{array}{l}45-54 \text { Years } \\
\text { Old }\end{array}$ & 1.156 & .578 & .449 & .007 & 1.075 & .617 & .435 & .008 & .297 & .036 & .851 & .001 \\
\hline$\geq 55$ Years Old & -2.145 & 1.749 & .189 & .019 & -.973 & .386 & .536 & .005 & -.859 & .278 & .600 & .005 \\
\hline Bachelor's & -1.863 & 1.641 & .204 & .018 & -1.376 & 1.016 & .317 & .014 & -.543 & .134 & .716 & .003 \\
\hline Master's & 1.066 & .567 & .454 & .006 & 1.730 & 1.753 & .190 & .023 & .409 & .076 & .784 & .001 \\
\hline Doctorate & 1.025 & .710 & .402 & .008 & -.343 & .090 & .765 & .001 & .099 & .006 & .938 & .000 \\
\hline Residency & 1.229 & .587 & .446 & .007 & -.113 & .006 & .939 & .000 & 1.648 & 1.039 & .313 & .020 \\
\hline Fellowship & 2.076 & .869 & .354 & .010 & -1.979 & .742 & .392 & .010 & -1.393 & .334 & .566 & .006 \\
\hline $\begin{array}{l}\text { Board } \\
\text { Certification }\end{array}$ & .037 & .001 & .977 & .000 & -.847 & .513 & .476 & .007 & -.810 & .372 & .544 & .007 \\
\hline $\begin{array}{l}\text { Board and } \\
\text { Manual } \\
\text { Therapy } \\
\text { Certification }\end{array}$ & -1.647 & .159 & .691 & .002 & 4.428 & .785 & .379 & .010 & 4.386 & .887 & .351 & .017 \\
\hline $\begin{array}{l}\text { No } \\
\text { Certification }\end{array}$ & -.694 & .322 & .572 & .004 & .364 & .100 & .753 & .001 & 1.427 & 1.266 & .266 & .024 \\
\hline $\begin{array}{l}\% \text { of Care } \\
\text { Provided by }\end{array}$ & -.007 & .099 & .754 & .001 & .001 & .005 & .945 & .000 & .002 & .010 & .922 & .000 \\
\hline
\end{tabular}




\begin{tabular}{|l|l|l|l|l|l|l|l|l|l|l|l|}
\hline $\begin{array}{l}\text { Other PT } \\
\text { Providers }\end{array}$ & & & & & & & & & & & \\
\hline
\end{tabular}

CLFS- Computerized Lumbar Functional Scale, FABQpa- Fear Avoidance Beliefs Questionnaire physical activity subscale, HCPAIRS-Health Care Providers' Pain and Impairment Relationship Scale, PABS-BM-Pain Attitudes and Beliefs Scale for Physiotherapists biomedical subscale, PABS-BPS-Pain Attitudes and Beliefs Scale for Physiotherapists biopsychosocial subscale, NPQ- Neurophysiology of Pain Questionnaire.

\section{Average CLFS Residual}

Simple linear regression results for CLFS residual scores are reported in Table 12. The three measures of beliefs were not significant predictors of CLFS residuals at the 5-patient and 20-patient cut-off, with $p$ values ranging from .100 (PABS-BPS) to .810 (PABS-BPS). Average FABQpa change $\left(B=-.131,95 \% \mathrm{Cl}[.000, .294], \mathrm{R}^{2}=.072, \mathrm{p}=.011\right)$, physical therapists with $\geq 25$ years of experience $\left(B=-2.501,95 \% \mathrm{Cl}[-4.777,-.225], \mathrm{R}^{2}=.51, \mathrm{p}=.032\right)$, and physical therapists who were 55 years old or older $\left(B=-2.802,95 \% \mathrm{Cl}[-5.431,-.173], R^{2}=.049, p=.037\right)$ were significant predictors of CLFS residual scores in the 5-patient cut-off sample. HC-PAIRS $(B=.114$, 95\% CI[.001, .228], $\left.\mathrm{R}^{2}=.050, \mathrm{p}=.048\right)$ and average FABQpa change $(\mathrm{B}=-.124,95 \% \mathrm{Cl}[-.219$, $.012], \mathrm{R}^{2}=.082, \mathrm{p}=.012$ ) were the only two predictors of CLFS residual scores in the 10 -patient cut-off sample. Average FABQpa change $\left(B=-.219,95 \% \mathrm{CI}[-.348,-.090], \mathrm{R}^{2}=.185, \mathrm{p}=.001\right)$ and $15-19$ years of experience $\left(B=3.151,95 \% \mathrm{Cl}[-.029,6.330], R^{2}=.072, p=.052\right)$ were significant predictors of CLFS residual scores in the 20-patient cut-off sample.

Table 12. Simple linear regression of average CLFS residual scores.

\begin{tabular}{|c|c|c|c|c|c|c|c|c|c|c|c|c|}
\hline & \multicolumn{12}{|c|}{$\begin{array}{l}\text { Simple Linear Regression } \\
\text { e Computerized Lumbar Functional Status Residual Score }\end{array}$} \\
\hline & \multicolumn{4}{|c|}{5 Patient Cut-off $n=92$} & \multicolumn{4}{|c|}{10 Patient Cut-off $n=78$} & \multicolumn{4}{|c|}{20 Patient Cut-off $n=54$} \\
\hline & $B$ & $F$ & $p$ & $R^{2}$ & $B$ & $F$ & $p$ & $R^{2}$ & $B$ & $F$ & $p$ & $R^{2}$ \\
\hline HC-PAIRS & .062 & 1.026 & .314 & .011 & .114 & 4.031 & .048 & .050 & .053 & .662 & .420 & .013 \\
\hline PABS-BM & .064 & .754 & .388 & .008 & .114 & 2.934 & .091 & .037 & .041 & .243 & .624 & .005 \\
\hline PABS-BPS & .179 & 2.767 & .100 & .030 & .025 & .058 & .810 & .001 & .092 & .616 & .436 & .012 \\
\hline
\end{tabular}




\begin{tabular}{|c|c|c|c|c|c|c|c|c|c|c|c|c|}
\hline NPQ & .098 & .180 & .672 & .002 & -.084 & .171 & .680 & .002 & .269 & 1.307 & .258 & .025 \\
\hline $\begin{array}{l}\text { Number of } \\
\text { Patients }\end{array}$ & -.011 & .165 & .685 & .002 & -.012 & .224 & .637 & .003 & -.015 & .252 & .618 & .005 \\
\hline $\begin{array}{l}\text { Average } \\
\text { Intake } \\
\text { FABQpa }\end{array}$ & -.014 & .061 & .805 & .001 & -.048 & .881 & .351 & .012 & -.001 & .000 & .993 & .000 \\
\hline $\begin{array}{l}\text { Average } \\
\text { FABQ pa } \\
\text { Change }\end{array}$ & -.131 & 6.673 & .011 & .072 & -.124 & 6.689 & .012 & .082 & -.219 & 11.587 & .001 & .185 \\
\hline $\begin{array}{l}\text { Average } \\
\text { Number of } \\
\text { Visits }\end{array}$ & .136 & .885 & .349 & .010 & .096 & .462 & .499 & .006 & -.139 & .645 & .426 & .012 \\
\hline $\begin{array}{l}\leq 4 \text { Yrs. } \\
\text { Experience }\end{array}$ & .025 & .000 & .984 & .000 & -1.153 & 1.000 & .321 & .013 & -.006 & .000 & .996 & .000 \\
\hline $\begin{array}{l}5-9 \text { Yrs. } \\
\text { Experience }\end{array}$ & 1.530 & 1.670 & .200 & .019 & .728 & .469 & .496 & .006 & -.466 & .123 & .727 & .002 \\
\hline $\begin{array}{l}\text { 10-14 Yrs. } \\
\text { of } \\
\text { Experience }\end{array}$ & -.344 & .038 & .847 & .000 & .670 & .153 & .697 & .002 & .946 & .284 & .597 & .006 \\
\hline $\begin{array}{l}15-19 \text { Yrs. } \\
\text { of } \\
\text { Experience }\end{array}$ & 1.947 & 1.357 & .247 & .015 & 1.445 & .932 & .337 & .012 & 3.151 & 3.958 & .052 & .072 \\
\hline $\begin{array}{l}20-24 \text { Yrs. } \\
\text { of } \\
\text { Experience }\end{array}$ & 1.764 & 1.220 & .272 & .014 & 1.383 & .947 & .334 & .013 & -.525 & .102 & .751 & .002 \\
\hline $\begin{array}{l}\geq 25 \text { Yrs. of } \\
\text { Experience }\end{array}$ & -2.501 & 4.767 & .032 & .051 & -1.510 & 2.057 & .156 & .027 & -1.400 & 1.444 & .235 & .028 \\
\hline $\begin{array}{l}18-34 \\
\text { Years Old }\end{array}$ & 1.341 & 1.737 & .191 & .019 & .035 & .001 & .971 & .000 & .042 & .002 & .969 & .000 \\
\hline $\begin{array}{l}35-44 \\
\text { Years Old }\end{array}$ & .188 & .023 & .880 & .000 & .288 & .065 & .800 & .001 & .412 & .116 & .735 & .002 \\
\hline $\begin{array}{l}45-54 \\
\text { Years Old }\end{array}$ & 1.067 & .720 & .399 & .008 & .821 & .553 & .459 & .007 & .866 & .458 & .501 & .009 \\
\hline $\begin{array}{l}\geq 55 \text { Years } \\
\text { Old }\end{array}$ & -2.802 & 4.486 & .037 & .049 & -1.496 & 1.422 & .237 & .019 & -1.491 & 1.286 & .262 & .025 \\
\hline Bachelor's & -1.876 & 2.447 & .121 & .027 & -1.303 & 1.408 & .239 & .019 & -.937 & .606 & .440 & .012 \\
\hline Master's & .999 & .728 & .396 & .008 & 1.429 & 1.838 & .179 & .024 & .891 & .548 & .463 & .011 \\
\hline Doctorate & 1.111 & 1.222 & .272 & .014 & -.167 & .033 & .857 & .000 & .034 & .001 & .974 & .000 \\
\hline Residency & 1.757 & 1.831 & .179 & .021 & .951 & .642 & .425 & .009 & 1.635 & 1.554 & .218 & .030 \\
\hline Fellowship & 2.065 & 1.300 & .257 & .015 & -.998 & .288 & .593 & .004 & -.841 & .182 & .671 & .004 \\
\hline $\begin{array}{l}\text { Board } \\
\text { Certificatio } \\
\text { n }\end{array}$ & .607 & .322 & .572 & .004 & -.192 & .040 & .841 & .001 & -.267 & .061 & .807 & .001 \\
\hline $\begin{array}{l}\text { Board and } \\
\text { Manual }\end{array}$ & -1.301 & .144 & .705 & .002 & 3.336 & .683 & .411 & .009 & 3.286 & .748 & .391 & .014 \\
\hline
\end{tabular}




\begin{tabular}{|l|l|l|l|l|l|l|l|l|l|l|l|l|}
\hline $\begin{array}{l}\text { Therapy } \\
\text { Certificatio } \\
\mathbf{n}\end{array}$ & & & & & & & & & & & & \\
\hline $\begin{array}{l}\text { No } \\
\text { Certificatio } \\
\mathbf{n}\end{array}$ & -.637 & .396 & .531 & .004 & -.230 & .061 & .805 & .001 & .731 & .492 & .486 & .010 \\
\hline $\begin{array}{l}\% \text { of Care } \\
\text { Provided } \\
\text { by Other } \\
\begin{array}{l}\text { PT } \\
\text { Providers }\end{array}\end{array}$ & -.021 & 1.451 & .232 & .016 & -.011 & .479 & .491 & .006 & -.006 & .118 & .732 & .002 \\
\hline
\end{tabular}

CLFS- Computerized Lumbar Functional Scale, FABQpa- Fear Avoidance Beliefs Questionnaire physical activity subscale, HCPAIRS-Health Care Providers' Pain and Impairment Relationship Scale, PABS-BM-Pain Attitudes and Beliefs Scale for Physiotherapists biomedical subscale, PABS-BPS-Pain Attitudes and Beliefs Scale for Physiotherapists biopsychosocial subscale, NPQ- Neurophysiology of Pain Questionnaire.

\section{Average Number of Visits}

Simple linear regression results for the average number of visits are reported in Table

13. The three measures of beliefs were not significant predictors of number of visits at the 5-

patient and 20-patient cut-off, with p values ranging from .340 (HC-PAIRS) to .921 (PABS-BPS).

An age of $35-44$ years old $\left(B=1.787,95 \% \mathrm{Cl}[.027,3.548], R^{2}=.044, p=.047\right)$ was the only

significant predictor of the average number of visits for the 5-patient cut-off sample. HC-PAIRS

$\left(B=.096,95 \% \mathrm{Cl}[.004, .188], \mathrm{R}^{2}=.054, \mathrm{p}=.041\right)$ and an age of $20-24$ years old $(\mathrm{B}=2.585,95 \%$

$\mathrm{Cl}[.300,4.870], \mathrm{R}^{2}=.064, \mathrm{p}=.027$ were significant predictors of average number of visits for the

10-patient cut-off sample. Average intake FABQpa $\left(B=-.098,95 \% \mathrm{Cl}[-.191,-.004], \mathrm{R}^{2}=.079, \mathrm{p}=\right.$

.041) was the only predictor of average number of visits for the 20-patient cut-off sample. 
Table 13. Simple linear regression of average number of visits.

\begin{tabular}{|c|c|c|c|c|c|c|c|c|c|c|c|c|}
\hline & \multicolumn{12}{|c|}{$\begin{array}{c}\text { Simple Linear Regression } \\
\text { Average Number of Visits }\end{array}$} \\
\hline & \multicolumn{4}{|c|}{5 Patient Cut-off $n=92$} & \multicolumn{4}{|c|}{10 Patient Cut-off $n=78$} & \multicolumn{4}{|c|}{20 Patient Cut-off $n=54$} \\
\hline & $B$ & $F$ & $p$ & $R^{2}$ & $B$ & $F$ & $p$ & $R^{2}$ & $B$ & $F$ & $p$ & $R^{2}$ \\
\hline HC-PAIRS & .042 & .919 & .340 & .010 & .096 & 4.325 & .041 & .054 & .046 & .802 & .375 & .015 \\
\hline PABS-BM & .049 & .863 & .355 & .009 & .106 & 3.895 & .052 & .049 & .016 & .060 & .807 & 001 \\
\hline PABS-BPS & .044 & .312 & .578 & .003 & .005 & .004 & .950 & .000 & .009 & .010 & .921 & .000 \\
\hline NPQ & -.099 & .358 & .551 & .004 & -.016 & .009 & .924 & .000 & .156 & .687 & .411 & .013 \\
\hline $\begin{array}{l}\text { Number of } \\
\text { Patients }\end{array}$ & -.016 & .680 & .412 & .007 & -.037 & 3.357 & .071 & .042 & -.014 & .354 & .554 & .007 \\
\hline $\begin{array}{l}\text { Average } \\
\text { Intake } \\
\text { FABQpa }\end{array}$ & -.070 & 3.100 & .082 & .035 & -.052 & 1.592 & .211 & .021 & -.098 & 4.401 & .041 & .079 \\
\hline $\begin{array}{l}\text { Average } \\
\text { FABQpa } \\
\text { Change }\end{array}$ & -.005 & .019 & .890 & .000 & -.007 & .033 & .856 & .000 & .044 & .599 & .442 & .012 \\
\hline $\begin{array}{l}\leq 4 \text { Yrs. } \\
\text { Experience }\end{array}$ & -5.003 & 1.057 & .307 & .012 & -4.197 & .624 & .432 & .008 & -8.825 & 2.446 & .124 & .046 \\
\hline $\begin{array}{l}\text { 5-9 Yrs. } \\
\text { Experience }\end{array}$ & -.325 & .138 & .711 & .002 & -.649 & .542 & .464 & .007 & -.807 & .585 & .448 & .011 \\
\hline $\begin{array}{l}10-14 \text { Yrs. } \\
\text { of } \\
\text { Experience }\end{array}$ & 2.457 & 3.728 & .057 & .041 & -5.143 & 1.444 & .233 & .019 & 2.258 & 2.660 & .109 & .050 \\
\hline $\begin{array}{l}\text { 15-19 Yrs. } \\
\text { of } \\
\text { Experience }\end{array}$ & -.630 & .262 & .610 & .003 & .868 & .486 & .488 & .007 & -.475 & .132 & .718 & .003 \\
\hline $\begin{array}{l}20-24 \text { Yrs. } \\
\text { of } \\
\text { Experience }\end{array}$ & 2.125 & 3.387 & .069 & .037 & 2.585 & 5.083 & .027 & .064 & -.663 & .257 & .614 & .005 \\
\hline $\begin{array}{l}\geq 25 \text { Yrs. of } \\
\text { Experience }\end{array}$ & -1.519 & 3.230 & .076 & .035 & -1.351 & 2.408 & .125 & .032 & -.832 & .792 & .378 & .015 \\
\hline $\begin{array}{l}18-34 \\
\text { Years Old }\end{array}$ & -.157 & .043 & .835 & .000 & -.085 & .012 & .913 & .000 & .464 & .289 & .593 & .006 \\
\hline $\begin{array}{l}35-44 \\
\text { Years Old }\end{array}$ & 1.787 & 4.070 & .047 & .044 & 1.313 & 2.004 & .161 & .026 & .325 & .114 & .737 & .002 \\
\hline $\begin{array}{l}45-54 \\
\text { Years Old }\end{array}$ & -.222 & .058 & .811 & .001 & -.280 & .093 & .761 & .001 & -.264 & .067 & .797 & .001 \\
\hline $\begin{array}{l}>55 \text { Years } \\
\text { Old }\end{array}$ & -1.533 & 2.455 & .121 & .027 & -1.120 & 1.154 & .286 & .015 & -.807 & .585 & .448 & .011 \\
\hline Bachelor's & -1.171 & 1.769 & .187 & .020 & -1.038 & 1.297 & .258 & .017 & -1.408 & 2.221 & .142 & .042 \\
\hline
\end{tabular}




\begin{tabular}{|c|c|c|c|c|c|c|c|c|c|c|c|c|}
\hline Master's & .406 & .224 & .637 & .003 & .825 & .879 & .351 & .012 & -.083 & .007 & .932 & .000 \\
\hline Doctorate & .556 & .567 & .453 & .006 & .103 & .018 & .894 & .000 & 1.104 & 1.831 & .182 & .035 \\
\hline Residency & .614 & .380 & .539 & .004 & .274 & .077 & .782 & .001 & .056 & .003 & .958 & .000 \\
\hline Fellowship & -.645 & .216 & .643 & .002 & -1.152 & .560 & .457 & .008 & -.816 & .271 & .605 & .005 \\
\hline $\begin{array}{l}\text { Board } \\
\text { Certificatio } \\
\text { n }\end{array}$ & -.267 & .116 & .735 & .001 & -.777 & .969 & .328 & .013 & -.766 & .796 & .377 & .015 \\
\hline $\begin{array}{l}\text { Board and } \\
\text { Manual } \\
\text { Therapy } \\
\text { Certificatio } \\
\text { n }\end{array}$ & -2.057 & .677 & .413 & .008 & -1.293 & .148 & .701 & .002 & -.769 & .064 & .802 & .001 \\
\hline $\begin{array}{l}\text { No } \\
\text { Certificatio } \\
\text { n }\end{array}$ & .334 & .203 & .654 & .002 & .181 & .055 & .815 & .001 & .389 & .219 & .642 & .004 \\
\hline $\begin{array}{l}\% \text { of Care } \\
\text { Provided } \\
\text { by Other } \\
\text { PT } \\
\text { Providers }\end{array}$ & .009 & .483 & .489 & .006 & .022 & 2.837 & .096 & .037 & .016 & 1.308 & .258 & .025 \\
\hline
\end{tabular}

FABQpa- Fear Avoidance Beliefs Questionnaire physical activity subscale, HC-PAIRS-Health Care Providers' Pain and Impairment Relationship Scale, PABS-BM-Pain Attitudes and Beliefs Scale for Physiotherapists biomedical subscale, PABS-BPS-Pain Attitudes and Beliefs Scale for Physiotherapists biopsychosocial subscale, NPQ- Neurophysiology of Pain Questionnaire.

\section{Multiple Linear Regression}

\section{Average CLFS Change}

Results from the CLFS change multiple linear regression are reported in Table 14. Seven potential predictors of CLFS change $(p \leq .25)$ were entered into a best subsets regression for the 5-patient cut-off sample. One hundred twenty-seven regressions were sorted by AIC and the model with the highest AIC (289.787) included PABS-BM, average FABQpa change, and age 55 years or older. Hierarchical multiple linear regression created a model that only included average FABQpa change. After entering number of patients in the first level, average FABQpa change $(\beta=-.186,95 \% \mathrm{Cl}[-.311,-.061], p=.004)$ was a significant predictor CLFS change $(p=.004)$ with an adjusted $\mathrm{R}^{2}$ of .079 . VIF scores were below 1.1 and Pearson Correlations were less than .207 , which indicated that multicollinearity was not present. Validation of the model with 2017 data also found that average FABQpa change was the only significant predictor. Pearson 
correlation of predicted values and actual values was $.310(p=.003)$ for 2018 data and .204 $(p=.051)$ for the 2017 data.

Five potential predictors of CLFS change $(p \leq .25)$ were entered into a best subsets regression for the 10-patient cut-off sample. Thirty-one regressions were sorted by AIC and the model with the highest AIC (231.202) included HC-PAIRS and average FABQpa change. Hierarchical multiple linear regression created a model that included both predictors. After entering number of patients in the first level, HC-PAIRS ( $\beta=.193,95 \% \mathrm{CI}[.059, .327], \mathrm{p}=.005)$ and average FABQpa change $(\beta=-.151,95 \% \mathrm{CI}[-.265,-.036], p=.010)$ were significant predictor CLFS change $(p=.010)$ with an adjusted $R^{2}$ of .161 . VIF scores were below 1.0 and Pearson Correlations were less than .152 , which indicated that multicollinearity was not present. Validation of the model with 2017 data also found that HC-PAIRS and average FABQpa change were significant predictors of CLFS. Pearson correlation of predicted values and actual values was .440 $(p=<.001)$ for 2018 data and $.238(p=.023)$ for the 2017 data.

Four potential predictors of CLFS change $(p \leq .25)$ were entered into a best subsets regression for the 20-patient cut-off sample. Fifteen regressions were sorted by AIC and the model with the highest AIC (146.872) included HC-PAIRS and average FABQpa change. Hierarchical multiple linear regression created a model that only included average FABQpa change. After entering number of patients in the first level, average $F A B Q p a$ change $(\beta=-.284$, $95 \% \mathrm{CI}[-.450,-.117], p=.001)$ was a significant predictor CLFS change $(p=.001)$ with an adjusted $\mathrm{R}^{2}$ of .176. VIF scores were below 1.06 and Pearson Correlations were less than .229, which indicated that multicollinearity was not present. Validation of the model with 2017 data also found that average FABQpa change was the only significant predictor of CLFS. Pearson 
correlation of predicted values and actual values was .458 $(p=.001)$ for 2018 data and .203 $(p=.053)$ for the 2017 data.

Table 14. Hierarchical multiple linear regression of average CLFS change

\begin{tabular}{|c|c|c|c|c|c|c|}
\hline & \multicolumn{6}{|c|}{$\begin{array}{l}\text { Computerized Lumbar Functional Scale Change Multiple Regression } \\
\text { 5-Patient Cut-Off Sample }\end{array}$} \\
\hline & \multirow[t]{2}{*}{ Coefficients } & \multirow[t]{2}{*}{$\beta$} & \multirow{2}{*}{$\begin{array}{c}\text { Standardized } \\
\beta\end{array}$} & \multicolumn{2}{|c|}{$95 \% \mathrm{Cl}$} & \multirow[t]{2}{*}{$P$} \\
\hline & & & & Lower Bound & Upper Bound & \\
\hline $\begin{array}{l}\text { Model } \\
C=15.130 \\
R^{2}=.100 \\
A^{2}=.079 \\
P=.004\end{array}$ & Average FABQpa Change & -.186 & -.308 & -.311 & -.061 & .004 \\
\hline \multicolumn{7}{|c|}{$\begin{array}{l}\text { Computerized Lumbar Functional Scale Change Multiple Regression } \\
\qquad 10 \text {-Patient Cut-Off Sample }\end{array}$} \\
\hline $\begin{array}{l}\text { Model } \\
\mathrm{C}=9.309\end{array}$ & HC-PAIRS & .193 & .304 & .059 & .327 & .005 \\
\hline $\begin{array}{l}\mathrm{R}^{2}=.194 \\
\mathrm{AR}^{2}=.161 \\
\mathrm{P}=.010\end{array}$ & Average FABQpa Change & -.151 & -.279 & -.265 & -.036 & .010 \\
\hline \multicolumn{7}{|c|}{$\begin{array}{l}\text { Computerized Lumbar Functional Scale Change Multiple Regression } \\
\text { 20-Patient Cut-Off Sample }\end{array}$} \\
\hline $\begin{array}{l}\text { Model } \\
C=14.249 \\
R^{2}=.208 \\
A^{2}=.176 \\
P=.001\end{array}$ & Average FABQpa Change & -.284 & -.443 & -.450 & -.117 & .001 \\
\hline
\end{tabular}

FABQpa- Fear Avoidance Beliefs Questionnaire physical activity subscale, HC-PAIRS-Health Care Providers' Pain and Impairment Relationship Scale, C-Constant, $\mathrm{AR}^{2}$-Adjusted $\mathrm{R}^{2}$

\section{Average CLFS Residual}

Results from the CLFS residual multiple regression are reported in Table 15. Twelve potential predictors of CLFS residual scores $(p \leq .25)$ were entered into a best subsets regression for the 5-patient cut-off sample. Four thousand ninety-five regressions were sorted by AIC and the model with the highest AIC (247.261) included PABS-BM, average FABQpa change, an age of 
55 years or more, residency training, and the percentage of other physical therapy providers delivering care to the physical therapists' patients. Hierarchical multiple linear regression created a model that included average FABQpa change and an age of 55 years or greater. After entering number of patients in the first level, average FABQpa change $(\beta=-.140,95 \% \mathrm{CI}[-.243$, $.037], p=.008)$ and an age of 55 years or greater $(\beta=-3.026,95 \% \mathrm{CI}[-5.726,-.325], p=.029$ were significant predictor CLFS residual scores $(p=.029)$ with an adjusted $R^{2}$ of .095 . VIF scores were below 1.05 and Pearson Correlations were less than .287, which indicated that multicollinearity was not present. Validation of the model with 2017 data also found that average FABQpa change was a significant predictor; however, age of 55 years or greater was not significant $(p=.311)$. Pearson correlation of predicted values and actual values was .339 $(p=.001)$ for 2018 data and $.164(p=.122)$ for the 2017 data.

Seven potential predictors of CLFS residual scores $(p \leq .25)$ were entered into a best subsets regression for the 10-patient cut-off sample. One hundred twenty-seven regressions were sorted by AIC and the model with the highest AIC (201.967) included PABS-BM, average FABQpa change, and an age of 55 years or greater. After entering number of patients in the first level, average FABQpa change $(\beta=-.123,95 \% \mathrm{CI}[-.221,-.025], \mathrm{p}=.015)$ was the only significant predictor CLFS residual scores ( $p=.015)$ with an adjusted $R^{2}$ of .057 . VIF scores were below 1.02 and Pearson Correlations were less than .119, which indicated that multicollinearity was not present. Validation of the model with 2017 data also found that average FABQpa change was the only significant predictor of CLSF residual scores. Pearson correlation of predicted values and actual values was $.288(p=.011)$ for 2018 data and $.188(p=.073)$ for the 2017 data. 
Five potential predictors of CLFS residual scores $(p \leq .25)$ were entered into a best subsets regression for the 20 -patient cut-off sample. Thirty-one regressions were sorted by AIC and the model with the highest AIC (124.931) included average FABQpa change, 15-19 years of experience and residency training. Hierarchical multiple linear regression created a model that included average FABQpa change, 15-19 years of experience and residency training. After entering number of patients in the first level, average FABQpa change $(\beta=-.232,95 \% \mathrm{CI}[-.360$, $.104], p=.001), 15-19$ years of experience $(\beta=3.630,95 \% \mathrm{Cl}[.634,6.625], p=.038$, and residency training $(\beta=2.485,95 \% \mathrm{Cl}[.138,4.832], \mathrm{p}=.038)$ were significant predictor CLFS residual scores $(p=.038)$ with an adjusted $R^{2}$ of .257 . VIF scores were below 1.08 and Pearson Correlations were less than .265 , which indicated that multicollinearity was not present. Validation of the model with 2017 data found that average FABQpa change was the only significant predictor of CLSF residual scores. Pearson correlation of predicted values and actual values was .568 $(p=.011)$ for 2018 data and $.250(p=.018)$ for the 2017 data.

Table 15. Hierarchical multiple linear regression for average CLFS residual scores.

\begin{tabular}{|c|c|c|c|c|c|c|}
\hline & \multicolumn{6}{|c|}{$\begin{array}{l}\text { Computerized Lumbar Functional Scale Residual Multiple Regression } \\
\text { 5-Patient Cut-Off Sample }\end{array}$} \\
\hline & \multirow[t]{2}{*}{ Coefficients } & \multirow[t]{2}{*}{$\beta$} & \multirow{2}{*}{$\begin{array}{l}\text { Standardized } \\
\beta\end{array}$} & \multicolumn{2}{|c|}{$95 \% \mathrm{Cl}$} & \multirow[t]{2}{*}{$P$} \\
\hline & & & & Lower Bound & Upper Bound & \\
\hline Model & Average FABQpa Change & -.140 & -.281 & -.243 & -.037 & .008 \\
\hline $\begin{array}{l}\mathrm{R}^{2}=.127 \\
\mathrm{AR}^{2}=.095 \\
\mathrm{p}=.029\end{array}$ & $\begin{array}{l}55 \text { Years of Age or } \\
\text { Older }\end{array}$ & -3.026 & -.237 & -5.726 & -3.250 & .029 \\
\hline \multicolumn{7}{|c|}{$\begin{array}{l}\text { Computerized Lumbar Functional Scale Residual Multiple Regression } \\
\qquad \text { 10-Patient Cut-Off Sample }\end{array}$} \\
\hline $\begin{array}{l}\text { Model } \\
\mathrm{C}=.888 \\
\mathrm{R}^{2}=.082 \\
\mathrm{AR}^{2}=.057\end{array}$ & Average FABQpa Change & -.123 & -.284 & -.221 & -.025 & .015 \\
\hline
\end{tabular}




\begin{tabular}{|c|c|c|c|c|c|c|}
\hline $\mathrm{P}=.015$ & & & & & & \\
\hline \multicolumn{7}{|c|}{ Computerized Lumbar Functional Scale Residual Multiple Regression } \\
\hline \multicolumn{7}{|c|}{ 20-Patient Cut-Off Sample } \\
\hline \multirow{4}{*}{$\begin{array}{l}\text { Model } \\
C=-1.816 \\
R^{2}=.316 \\
A R^{2}=.257 \\
P=.038\end{array}$} & Average FABQpa Change & -.232 & -.454 & -.360 & -.104 & .001 \\
\hline & & & & & & \\
\hline & $\begin{array}{l}\text { 15-19 Years of } \\
\text { Experience }\end{array}$ & 3.630 & .310 & .634 & 6.625 & .019 \\
\hline & Residency Training & 2.485 & .262 & .138 & 4.832 & .038 \\
\hline
\end{tabular}

FABQpa- Fear Avoidance Beliefs Questionnaire physical activity subscale, C-Constant, AR ${ }^{2}$-Adjusted R²

\section{Average Number of Visits}

Results from the average number of visits multiple regression are reported in Table 16.

Six potential predictors of average number of visits $(p \leq .25)$ were entered into a best subsets regression for the 5-patient cut-off sample. Sixty-three regressions were sorted by AIC and the model with the highest AIC (208.429) included average FABQpa intake scores, 10-14 years of experience, and 20-24 years of experience. Hierarchical multiple linear regression created a model that included $10-14$ and 20-24 years of experience. After entering number of patients in the first level, $10-14$ years of experience $(\beta=2.972,95 \% \mathrm{Cl}[.403,5.541], p=.024)$ and $20-24$ years of experience $(\beta=2.379,95 \% \mathrm{Cl}[.084,4.674], p=.042$ were significant predictors of average number of visits ( $p=.042)$ with an adjusted $R^{2}$ of .069 . VIF scores were below 1.04 and Pearson Correlations were less than .193, which indicated that multicollinearity was not present. Validation of the model with 2017 data found that the coefficients included in the 2018 model were not significant predictors of average number of visits in the 2017 data. Pearson correlation of predicted values and actual values was .319 $(p=.002)$ for 2018 data and $.152(p=.152)$ for the 2017 data. 
Seven potential predictors of average number of visits $(p \leq .25)$ were entered into a best subsets regression for the 10-patient cut-off sample. One hundred twenty-seven regressions were sorted by AIC and the model with the highest AIC (175.631) included HC-PAIRS, average intake FABQpa and 20-24 years of experience. Hierarchical multiple linear regression created a model that included HC-PAIRS and average intake FABQpa. After entering number of patients in the first level, HC-PAIRS $(\beta=.130,95 \% \mathrm{CI}[.037, .223], p=.007)$ and intake FABQpa $(\beta=-.093,95 \%$ $\mathrm{CI}[-.174,-.011], \mathrm{p}=.027$ were significant predictors of average number of visits $(p=.027)$ with an adjusted $R^{2}$ of .128. VIF scores were below 1.10 and Pearson Correlations were less than .250, which indicated that multicollinearity was not present. Validation of the model with 2017 data found that the coefficients included in the 2018 model were not significant predictors of average number of visits in the 2017 data. Pearson correlation of predicted values and actual values was $.405(p<.001)$ for 2018 data and $.118(p=.263)$ for the 2017 data.

Five potential predictors of average number of visits $(p \leq .25)$ were entered into a best subsets regression for the 20-patient cut-off sample. Thirty-one regressions were sorted by AIC and the model with the highest AIC (111.863) included average intake FABQpa, less than 4 years of experience, and 10-14 years of experience. Hierarchical multiple linear regression created a model that only included average intake FABQpa scores. After entering number of patients in the first level, intake FABQpa $(\beta=-.106,95 \% \mathrm{CI}[-.202,-.011], \mathrm{p}=.030)$ was a significant predictor of average number of visits $(p=.030)$ with an adjusted $R^{2}$ of .062 . VIF scores were below 1.04 and Pearson Correlations were less than .298, which indicated that multicollinearity was not present. Validation of the model with 2017 data found that the coefficients included in the 2018 model were not significant predictors of average number of 
visits in the 2017 data. Pearson correlation of predicted values and actual values was .362 $(p=.008)$ for 2018 data and $.152(p=.149)$ for the 2017 data.

Table 16. Hierarchical multiple linear regression of average number of visits.

\begin{tabular}{|c|c|c|c|c|c|c|}
\hline & \multicolumn{6}{|c|}{$\begin{array}{l}\text { Average Number of Visits Multiple Regression } \\
\text { 5-Patient Cut-Off Sample }\end{array}$} \\
\hline & \multirow[t]{2}{*}{ Coefficients } & \multirow[t]{2}{*}{$\beta$} & \multirow{2}{*}{$\begin{array}{c}\text { Standardized } \\
\beta\end{array}$} & \multicolumn{2}{|c|}{$95 \% \mathrm{Cl}$} & \multirow[t]{2}{*}{$P$} \\
\hline & & & & Lower Bound & Upper Bound & \\
\hline \multirow{2}{*}{$\begin{array}{l}\text { Model } \\
C=10.086 \\
R^{2}=.102 \\
A^{2}=.069 \\
p=.042\end{array}$} & $\begin{array}{l}\text { 10-14 Years of } \\
\text { Experience }\end{array}$ & 2.972 & .246 & .403 & 5.541 & .024 \\
\hline & $\begin{array}{l}20-24 \text { Years of } \\
\text { Experience }\end{array}$ & 2.379 & .217 & .084 & 4.674 & .042 \\
\hline \multicolumn{7}{|c|}{$\begin{array}{l}\text { Average Number of Visits Multiple Regression } \\
10-\text { Patient Cut-Off Sample }\end{array}$} \\
\hline $\begin{array}{l}\text { Model } \\
\mathrm{C}=12.058\end{array}$ & HC-PAIRS & .130 & .313 & .037 & .223 & .007 \\
\hline $\begin{array}{l}\mathrm{R}^{2}=.163 \\
\mathrm{AR}^{2}=.128 \\
\mathrm{P}=.060\end{array}$ & Average Intake FABQpa & -.093 & -.257 & -.174 & -.011 & .027 \\
\hline \multicolumn{7}{|c|}{$\begin{array}{c}\text { Average Number of Visits Multiple Regression } \\
\text { 20-Patient Cut-Off Sample }\end{array}$} \\
\hline $\begin{array}{l}\text { Model } \\
C=15.685 \\
R^{2}=.314 \\
A^{2}=.062 \\
P=.030\end{array}$ & Average FABQpa Change & -.106 & -.309 & -.202 & -.011 & .030 \\
\hline
\end{tabular}

FABQpa- Fear Avoidance Beliefs Questionnaire physical activity subscale, HC-PAIRS-Health Care Providers' Pain and Impairment Relationship Scale, C-Constant, $\mathrm{AR}^{2}$-Adjusted $\mathrm{R}^{2}$

\section{Simple Logistic Regression}

\section{Average CLFS Change}

Simple logistic regression results for CLFS change are reported in Table 17. In the 5patient cut-off sample, 11 physical therapists had an average CLFS change score in the minimum to no change category, 36 had an average change score in the moderate change category, and 45 physical therapists were categorized into the significant change category. The only significant predictor of CLFS change for the 5-patient cut-off sample was 4-years of 
experience or less. This variable predicted a moderate change in CLFS scores $(\beta=-1.642$, $\mathrm{SE}=.774$, Walds $\left.\mathrm{X}^{2}=4.503, \mathrm{p}=.034\right)$ with an odds ratio of $.194(95 \% \mathrm{Cl}[.042, .882])$. In the $10-$ patient cut-off sample, seven physical therapists had an average CLFS change score in the minimum to no change category, 32 had an average change score in the moderate change category, and 39 physical therapists had an average CLFS change score in the significant change category. Having 4-years of experience or less was the only significant predictor for CLFS change scores for the 10-patient cut-off as well. This variable predicted a moderate change in average CLFS scores $\left(\beta=-2.234, S E=.932\right.$, Walds $\left.X^{2}=5.741, p=.017\right)$ with an odds ratio of .107 $(95 \% \mathrm{Cl}[.017, .666])$ and it predicted a significant change in CLFS scores $(\beta=-1.743, \mathrm{SE}=.872$ Walds $\left.X^{2}=4.000, p=.046\right)$ with an odds ratio of $\left..175995 \% \mathrm{Cl}[.032, .966]\right)$. In the 20-patient cutoff sample, five physical therapists had an average CLFS change score in the minimum to no change category, 19 had an average change score in the moderate change category, and 30 physical therapists had an average CLFS change score in the significant change category. The only significant predictor of CLFS change for the 20-patient cut-off sample was 4-years of experience or less. This variable predicted a moderate change in CLFS scores $(\beta=2.546$, $\mathrm{SE}=1.180$, Walds $\left.\mathrm{X}^{2}=4.654, \mathrm{p}=.031\right)$ with an odds ratio of $.078(95 \% \mathrm{Cl}[.008, .792])$. 
Table 17. Simple logistic regression of average CLFS scores.

\begin{tabular}{|c|c|c|c|c|c|c|c|c|c|c|c|c|}
\hline & \multicolumn{12}{|c|}{ 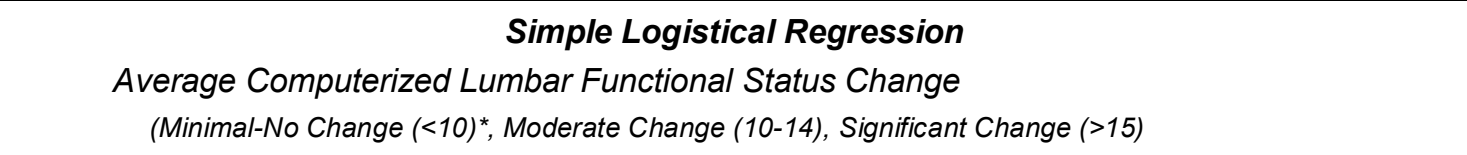 } \\
\hline & \multicolumn{4}{|c|}{$\begin{array}{l}5 \text { Patient Cut-off ( } n=92) \\
\text { Min } n=11, \operatorname{Mod} n=36, \operatorname{Sig} n=45\end{array}$} & \multicolumn{4}{|c|}{$\begin{array}{l}10 \text { Patient Cut-off ( } n=78) \\
\operatorname{Min} n=7, \operatorname{Mod} n=32, \operatorname{Sig} n=39\end{array}$} & \multicolumn{4}{|c|}{$\begin{array}{l}20 \text { Patient Cut-off ( } n=54) \\
\operatorname{Min} n=5, \operatorname{Mod} n=19, \operatorname{Sig} n=30\end{array}$} \\
\hline & $B$ & $S E$ & $p$ & OR & $B$ & $S E$ & $p$ & OR & $B$ & $S E$ & $p$ & OR \\
\hline $\begin{array}{l}\text { HC-PAIRS } \\
\text { Moderate }\end{array}$ & -.022 & .044 & .618 & .978 & -.014 & .059 & .816 & .986 & .000 & .073 & .995 & 1.000 \\
\hline $\begin{array}{l}\text { HC-PAIRS } \\
\text { Significant }\end{array}$ & .029 & .042 & .486 & 1.030 & .063 & .057 & .268 & 1.065 & .074 & .070 & .290 & 1.077 \\
\hline $\begin{array}{l}\text { PABS-BM } \\
\text { Moderate }\end{array}$ & .011 & .055 & .843 & 1.011 & .030 & .070 & .665 & 1.031 & .025 & .092 & .785 & 1.025 \\
\hline $\begin{array}{l}\text { PABS-BM } \\
\text { Significant }\end{array}$ & .058 & .053 & .277 & 1.060 & .090 & .070 & .194 & 1.095 & .070 & .088 & .430 & 1.072 \\
\hline $\begin{array}{l}\text { PABS-BPS } \\
\text { Moderate }\end{array}$ & .036 & .075 & .629 & 1.037 & -.056 & .098 & .566 & .945 & -.016 & .121 & .893 & .984 \\
\hline $\begin{array}{l}\text { PABS-BPS } \\
\text { Significant }\end{array}$ & .035 & .073 & .634 & 1.035 & -.100 & .097 & .303 & .905 & -.078 & .116 & .503 & .925 \\
\hline $\begin{array}{l}\text { NPQ } \\
\text { Moderate }\end{array}$ & .179 & .156 & .252 & 1.196 & .299 & .186 & .109 & 1.349 & .169 & .215 & .434 & 1.184 \\
\hline $\begin{array}{l}\text { NPQ } \\
\text { Significant }\end{array}$ & .038 & .137 & .779 & 1.039 & .042 & .153 & .783 & 1.043 & .082 & .194 & .671 & 1.086 \\
\hline $\begin{array}{l}\text { Number of } \\
\text { Patients } \\
\text { Moderate }\end{array}$ & .018 & .025 & .468 & 1.018 & -.005 & .026 & .863 & .995 & .013 & .037 & .715 & 1.014 \\
\hline $\begin{array}{l}\text { Number of } \\
\text { Patients } \\
\text { Significant }\end{array}$ & .033 & .024 & .171 & 1.033 & .016 & .025 & .511 & 1.017 & .024 & .036 & .496 & 1.024 \\
\hline $\begin{array}{l}\text { Average } \\
\text { Intake } \\
\text { FABQ } \\
\text { Moderate }\end{array}$ & .027 & .044 & .541 & 1.027 & .004 & .043 & .934 & 1.004 & .044 & .101 & .660 & 1.045 \\
\hline $\begin{array}{l}\text { Average } \\
\text { Intake } \\
\text { FABQ } \\
\text { Significant }\end{array}$ & .007 & .044 & .866 & 1.007 & -.018 & .044 & .694 & .983 & .061 & .099 & .542 & 1.062 \\
\hline $\begin{array}{l}\text { Average } \\
\text { FABQ } \\
\text { Change } \\
\text { Moderate }\end{array}$ & -.027 & .040 & .502 & .973 & .002 & .048 & .972 & 1.002 & -.051 & .085 & .548 & .950 \\
\hline $\begin{array}{l}\text { Average } \\
\text { FABQ } \\
\text { Change } \\
\text { Significant }\end{array}$ & -.056 & .040 & .162 & .945 & -.033 & .047 & .480 & .967 & -.115 & .083 & .165 & .891 \\
\hline
\end{tabular}




\begin{tabular}{|c|c|c|c|c|c|c|c|c|c|c|c|c|}
\hline $\begin{array}{l}\text { Average } \\
\text { Number of } \\
\text { Visits } \\
\text { Moderate }\end{array}$ & -.060 & .100 & .546 & .941 & -.151 & .122 & .215 & .860 & -.218 & .172 & .204 & .804 \\
\hline $\begin{array}{l}\text { Average } \\
\text { Number of } \\
\text { Visits } \\
\text { Significant }\end{array}$ & .001 & .095 & .989 & 1.001 & -.056 & .113 & .621 & .946 & -.121 & .161 & .450 & .886 \\
\hline $\begin{array}{l}\leq 4 \text { Yrs. } \\
\text { Experience } \\
\text { Moderate }\end{array}$ & -1.642 & .774 & .034 & .194 & -2.234 & .932 & .017 & .107 & -2.546 & 1.180 & .031 & .078 \\
\hline $\begin{array}{l}\leq 4 \text { Yrs. } \\
\text { Experience } \\
\text { Significant }\end{array}$ & -1.147 & .712 & .107 & .318 & -1.743 & .872 & .046 & .175 & -1.551 & 1.011 & .125 & .212 \\
\hline $\begin{array}{l}5-9 \text { Yrs. } \\
\text { Experience } \\
\text { Moderate }\end{array}$ & 1 & 1 & 1 & 1 & 1 & 1 & 1 & 1 & 1 & 1 & 1 & 1 \\
\hline $\begin{array}{l}\text { 5-9 Yrs. } \\
\text { Experience } \\
\text { Significant }\end{array}$ & 1 & 1 & 1 & 1 & 1 & 1 & 1 & 1 & 1 & 1 & 1 & 1 \\
\hline $\begin{array}{l}10-14 \text { Yrs. } \\
\text { of } \\
\text { Experience } \\
\text { Moderate }\end{array}$ & -.095 & 1.210 & .937 & .909 & 1 & 1 & 1 & 1 & 1 & 1 & 1 & 1 \\
\hline $\begin{array}{l}10-14 \text { Yrs. } \\
\text { of } \\
\text { Experience } \\
\text { Significant }\end{array}$ & .025 & 1.173 & .983 & 1.026 & 1 & 1 & 1 & 1 & 1 & 1 & 1 & 1 \\
\hline $\begin{array}{l}\text { 15-19 Yrs. } \\
\text { of } \\
\text { Experience } \\
\text { Moderate }\end{array}$ & 1 & 1 & 1 & 1 & 1 & 1 & 1 & 1 & 1 & 1 & 1 & 1 \\
\hline $\begin{array}{l}\text { 15-19 Yrs. } \\
\text { of } \\
\text { Experience } \\
\text { Significant }\end{array}$ & 1 & 1 & 1 & 1 & 1 & 1 & 1 & 1 & 1 & 1 & 1 & 1 \\
\hline $\begin{array}{l}20-24 \text { Yrs. } \\
\text { of } \\
\text { Experience } \\
\text { Moderate }\end{array}$ & -.531 & 1.276 & .678 & .588 & -.916 & 1.304 & .482 & .400 & 1 & 1 & 1 & 1 \\
\hline $\begin{array}{l}20-24 \text { Yrs. } \\
\text { of } \\
\text { Experience } \\
\text { Significant }\end{array}$ & .665 & 1.127 & .555 & 1.944 & .150 & 1.169 & .898 & 1.161 & 1 & 1 & 1 & 1 \\
\hline $\begin{array}{l}\geq 25 \text { Yrs. of } \\
\text { Experience } \\
\text { Moderate }\end{array}$ & .410 & .761 & .590 & 1.507 & .270 & .916 & .768 & 1.310 & -.134 & 1.029 & .897 & .875 \\
\hline $\begin{array}{l}\geq 25 \text { Yrs. of } \\
\text { Experience } \\
\text { Significant }\end{array}$ & -.838 & .807 & .299 & .432 & -.726 & .948 & .444 & .484 & -1.163 & 1.037 & .262 & .313 \\
\hline
\end{tabular}




\begin{tabular}{|c|c|c|c|c|c|c|c|c|c|c|c|c|}
\hline $\begin{array}{l}\text { 18-34 Years } \\
\text { Old } \\
\text { Moderate }\end{array}$ & -.388 & .698 & .578 & .678 & -.799 & .847 & .346 & .450 & -1.435 & 1.051 & .172 & .238 \\
\hline $\begin{array}{l}\text { 18-34 Years } \\
\text { Old } \\
\text { Significant }\end{array}$ & -.051 & .679 & .940 & .950 & -.671 & .834 & .421 & .511 & -.898 & .990 & .364 & .407 \\
\hline $\begin{array}{l}\text { 35-44 Years } \\
\text { Old } \\
\text { Moderate }\end{array}$ & .083 & .888 & .926 & 1.086 & .325 & 1.171 & .781 & 1.385 & 1 & 1 & 1 & 1 \\
\hline $\begin{array}{l}\text { 35-44 Years } \\
\text { Old } \\
\text { Significant }\end{array}$ & .310 & .861 & .719 & 1.364 & .657 & 1.146 & .567 & 1.929 & 1 & 1 & 1 & 1 \\
\hline $\begin{array}{l}\text { 45-54 Years } \\
\text { Old } \\
\text { Moderate }\end{array}$ & 1 & 1 & 1 & 1 & 1 & 1 & 1 & 1 & 1 & 1 & 1 & 1 \\
\hline $\begin{array}{l}\text { 45-54 Years } \\
\text { Old } \\
\text { Significant }\end{array}$ & 1 & 1 & 1 & 1 & 1 & 1 & 1 & 1 & 1 & 1 & 1 & 1 \\
\hline $\begin{array}{l}>55 \text { Years } \\
\text { Old } \\
\text { Moderate }\end{array}$ & -.441 & .797 & .581 & .644 & -.770 & .968 & .426 & .463 & -1.269 & 1.109 & .253 & .281 \\
\hline $\begin{array}{l}>55 \text { Years } \\
\text { Old } \\
\text { Significant }\end{array}$ & -1.047 & .827 & .206 & .351 & -.940 & .965 & .330 & .391 & -1.163 & 1.037 & .262 & .313 \\
\hline $\begin{array}{l}\text { Bachelor's } \\
\text { Moderate }\end{array}$ & -.396 & .729 & .587 & .673 & -.651 & .859 & .449 & .522 & -.368 & 1.038 & .723 & .692 \\
\hline $\begin{array}{l}\text { Bachelor's } \\
\text { Significant }\end{array}$ & -1.260 & .766 & .100 & .284 & -1.569 & .903 & .082 & .208 & -1.163 & 1.037 & .262 & .312 \\
\hline $\begin{array}{l}\text { Master's } \\
\text { Moderate }\end{array}$ & 1.204 & 1.117 & .281 & 3.333 & 1 & 1 & $T$ & 1 & 1 & $T$ & 1 & $T$ \\
\hline $\begin{array}{l}\text { Master's } \\
\text { Significant }\end{array}$ & 1.354 & 1.103 & .220 & 3.871 & 1 & 1 & 1 & 1 & 1 & 1 & 1 & 1 \\
\hline $\begin{array}{l}\text { Doctorate } \\
\text { Moderate }\end{array}$ & .071 & .691 & .918 & 1.074 & -.413 & .842 & .624 & .662 & -.944 & 1.029 & .359 & .389 \\
\hline $\begin{array}{l}\text { Doctorate } \\
\text { Significant }\end{array}$ & .511 & .680 & .452 & 1.667 & -.016 & .833 & .985 & .984 & -.057 & .988 & .954 & .944 \\
\hline $\begin{array}{l}\text { Residency } \\
\text { Moderate }\end{array}$ & -1.012 & .994 & .309 & .364 & -1.352 & 1.033 & .191 & .259 & -1.504 & 1.518 & .322 & .222 \\
\hline $\begin{array}{l}\text { Residency } \\
\text { Significant }\end{array}$ & .192 & .869 & .825 & 1.212 & -.219 & .920 & .812 & .804 & .421 & 1.193 & .724 & 1.524 \\
\hline $\begin{array}{l}\text { Fellowship } \\
\text { Moderate }\end{array}$ & -.201 & 1.214 & .869 & .818 & -.477 & 1.239 & .700 & .621 & -.754 & 1.345 & .575 & .471 \\
\hline $\begin{array}{l}\text { Fellowship } \\
\text { Moderate }\end{array}$ & -.393 & 1.212 & .746 & .675 & -1.792 & 1.481 & .226 & .167 & -1.946 & 1.512 & .198 & .143 \\
\hline $\begin{array}{l}\text { Board } \\
\text { Certification } \\
\text { Moderate }\end{array}$ & 1.052 & .853 & .218 & 2.864 & .665 & .909 & .465 & 1.944 & .087 & 1.024 & .932 & 1.091 \\
\hline
\end{tabular}




\begin{tabular}{|c|c|c|c|c|c|c|c|c|c|c|c|c|}
\hline $\begin{array}{l}\text { Board } \\
\text { Certification } \\
\text { Significant }\end{array}$ & .776 & .847 & .360 & 2.172 & .182 & .907 & .841 & 1.200 & -.393 & .997 & .693 & .675 \\
\hline $\begin{array}{l}\text { Board and } \\
\text { Manual } \\
\text { Therapy } \\
\text { Certification } \\
\text { Moderate }\end{array}$ & 1 & 1 & 1 & 1 & 1 & 1 & 1 & 1 & 1 & 1 & 1 & 1 \\
\hline $\begin{array}{l}\text { Board and } \\
\text { Manual } \\
\text { Therapy } \\
\text { Certification } \\
\text { Significant }\end{array}$ & -1.435 & 1.457 & .325 & .238 & 1 & 1 & 1 & 1 & 1 & 1 & 1 & 1 \\
\hline $\begin{array}{l}\text { No } \\
\text { Certification } \\
\text { Moderate }\end{array}$ & -.560 & .710 & .431 & .571 & -1.041 & .909 & .252 & .353 & -.724 & 1.024 & .480 & .485 \\
\hline $\begin{array}{l}\text { No } \\
\text { Certification } \\
\text { Significant }\end{array}$ & -.326 & .698 & .640 & .722 & -.533 & .901 & .554 & .587 & .087 & .990 & .930 & 1.091 \\
\hline $\begin{array}{l}\% \text { of Care } \\
\text { Provided by } \\
\text { Other PT } \\
\text { Providers } \\
\text { Moderate }\end{array}$ & -.027 & .013 & .003 & .973 & -.028 & .015 & .059 & .973 & -.017 & .018 & .324 & .983 \\
\hline $\begin{array}{l}\% \text { of Care } \\
\text { Provided by } \\
\text { Other PT } \\
\text { Providers } \\
\text { Significant }\end{array}$ & -.012 & .012 & .331 & .989 & -.009 & .014 & .521 & .991 & .003 & .016 & .840 & 1.003 \\
\hline
\end{tabular}

FABQpa- Fear Avoidance Beliefs Questionnaire physical activity subscale, HC-PAIRS-Health Care Providers' Pain and Impairment Relationship Scale, PABS-BM-Pain Attitudes and Beliefs Scale for Physiotherapists biomedical subscale, PABS-BPS-Pain Attitudes and Beliefs Scale for Physiotherapists biopsychosocial subscale, NPQ- Neurophysiology of Pain Questionnaire. *Reference standard=Minimal Change, $\backslash$ Contains cells with zero frequencies and could not be calculated

\section{Average CLFS Residual}

Simple logistic regression results for CLFS residual scores are reported in Table 18. In the 5-patient cut-off sample, 36 physical therapists had a CLFS residual score in the less than expected category, and 56 had residual scores in the more than expected category. In the 20patient cut-off sample, 19 physical therapists had a CLFS residual score in the less than expected category, and 35 had residual scores in the more than expected category. There were no significant predictors of CLFS residual scores for the 5-patient cut-off sample or the 20patient cut-off sample. In the 10-patient cut-off sample, 30 physical therapists had a CLFS 
residual score in the less than expected category, and 48 had residual scores in the more than expected category. HC-PAIRS was a significant predictor of CLFS residual scores $(\beta=.067$, $\mathrm{SE}=.033$, Walds $\left.\mathrm{X}^{2}=4.119, \mathrm{p}=.042\right)$ at the 10 -patient cut-off with an odds ratio of 1.070 (95\% $\mathrm{CI}[1.002,1.141])$. Receiver operating characteristic (ROC) curve for HC-PAIRS produced an area under the curve (AUC) of .620. (Table 19) Sensitivity and specificity are maximized at an HCPAIRS cut off score of 30.50, with values of .564 and .641 respectively. (Figure 1 and Table 20)

Table 18. Simple logistic regression of average CLFS residual.

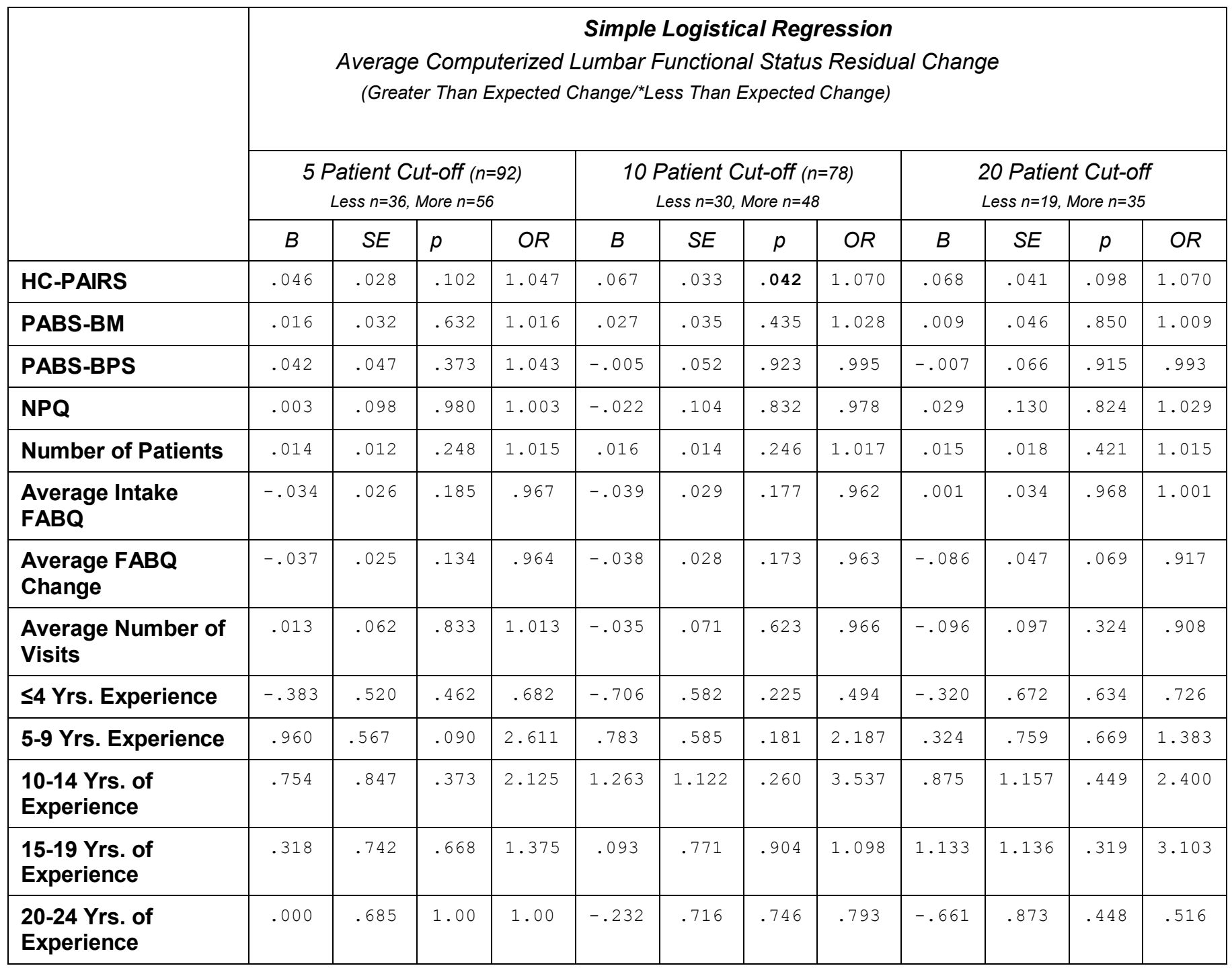




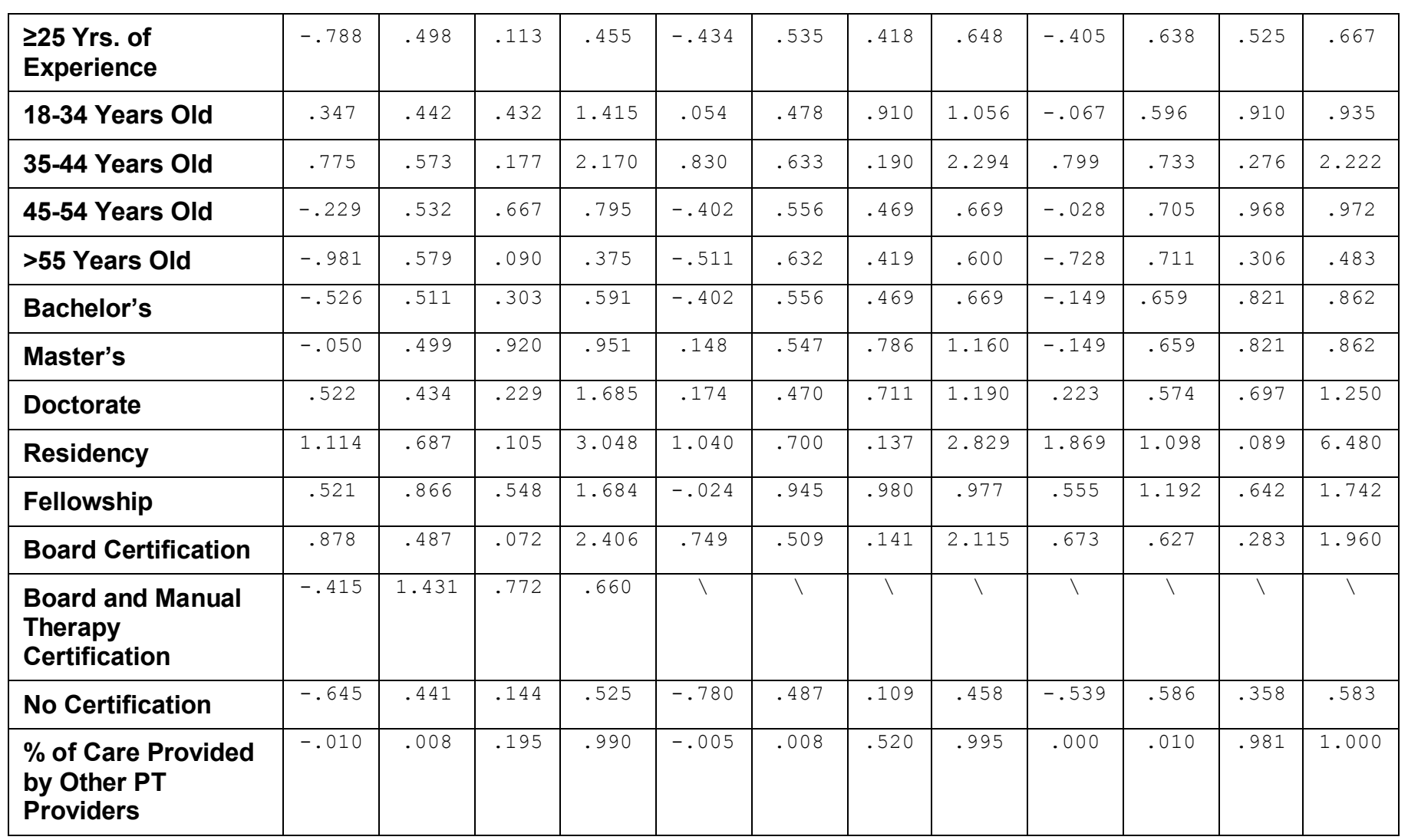

FABQpa- Fear Avoidance Beliefs Questionnaire physical activity subscale, HC-PAIRS-Health Care Providers' Pain and Impairment Relationship Scale, PABS-BM-Pain Attitudes and Beliefs Scale for Physiotherapists biomedical subscale, PABS-BPS-Pain Attitudes and Beliefs Scale for Physiotherapists biopsychosocial subscale, NPQ- Neurophysiology of Pain Questionnaire. *Reference standard= Less than expected change, $\backslash$ Contains cells with zero frequencies and could not be calculated

Table 19. Receiver operating characteristic curve for HC-PAIRS ability to predict CLFS residual.

\begin{tabular}{|c|c|c|c|c|c|c|c|}
\hline \multirow{3}{*}{ Category } & \multicolumn{7}{|c|}{$\begin{array}{c}\text { Discriminatory Ability HC-PAIRS } \\
\text { CLFS Residual 10-Patient Cut-Off Sample }\end{array}$} \\
\hline & \multirow[t]{2}{*}{ Category Cut Off } & \multirow[t]{2}{*}{$N(\%)$} & \multirow[t]{2}{*}{$A \cup C$} & \multirow[t]{2}{*}{ Std.Error } & \multicolumn{2}{|c|}{$95 \% \mathrm{Cl}$} & \multirow[t]{2}{*}{$P$} \\
\hline & & & & & $\begin{array}{l}\text { Lower } \\
\text { Bound }\end{array}$ & $\begin{array}{l}\text { Upper } \\
\text { Bound }\end{array}$ & \\
\hline $\begin{array}{l}\text { Great Than } \\
\text { Predicted } \\
\text { Change }\end{array}$ & CLFS Residual >0 & $39(50 \%)$ & .620 & .063 & .496 & .744 & .068 \\
\hline
\end{tabular}

CLFS- Computerized Lumbar Functional Scale, HC-PAIRS-Health Care Providers' Pain and Impairment Relationship Scale 
Figure 1: Receiver operating characteristic curve for HC-PAIRS ability to predict CLFS residual

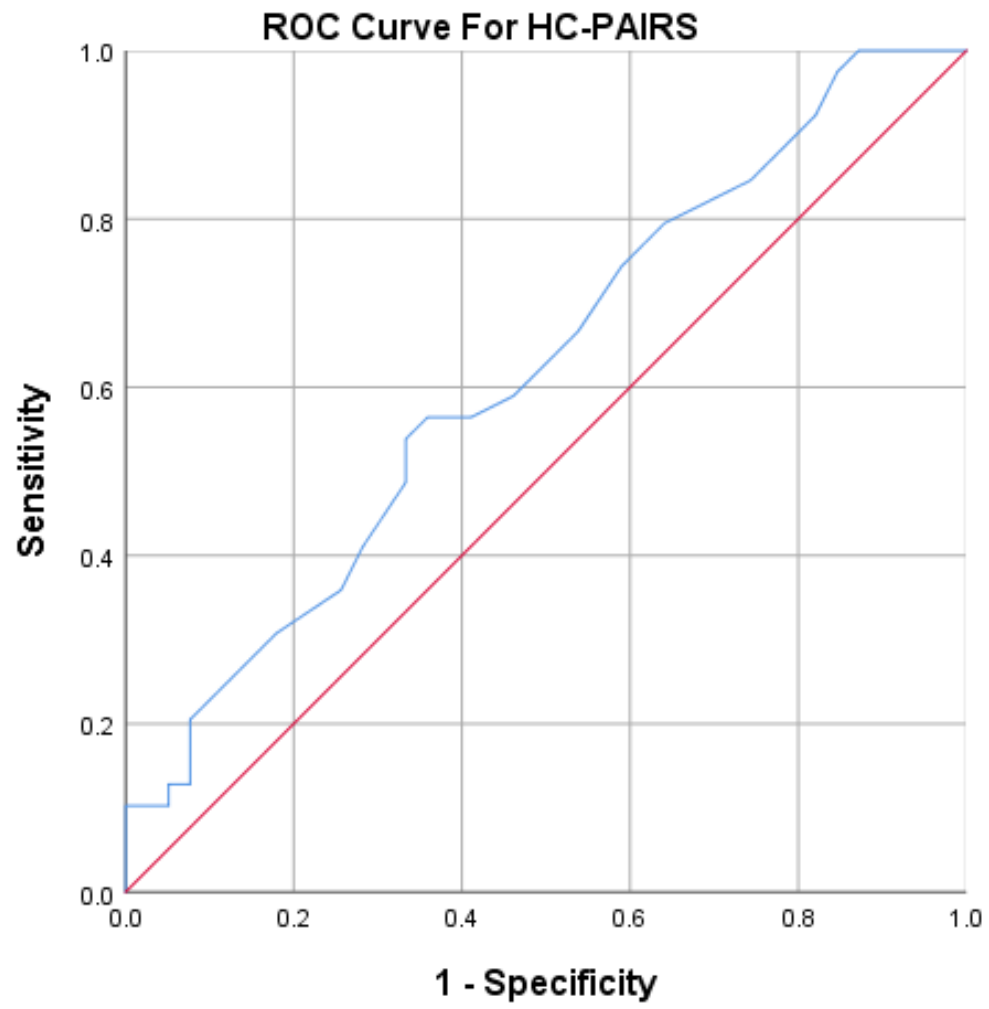

AUC $=.620$, For Prediction of CLFS Residual Scores, 10 Patient Cut-Off Sample

CLFS- Computerized Lumbar Functional Scale, HC-PAIRS-Health Care Providers' Pain and Impairment Relationship Scale

Table 20. Receiver operating characteristic curve coordinates for HC-PAIRS ability to predict CLFS residual.

\begin{tabular}{|c|c|c|}
\hline \multicolumn{3}{|c|}{ Coordinates of the Curve } \\
\hline \multicolumn{3}{|c|}{ CLFS Residual >1, 10-Patient Cut-Off Sample } \\
\hline $\begin{array}{c}\text { HC-PAIRS } \\
\text { Score }\end{array}$ & Sensitivity & 1 - Specificity \\
\hline 17.00 & 1.000 & 1.000 \\
\hline 19.00 & 1.000 & .923 \\
\hline 20.50 & 1.000 & .872 \\
\hline 21.50 & .974 & .846 \\
\hline 22.50 & .923 & .821 \\
\hline 23.50 & .897 & .795 \\
\hline 24.50 & .846 & .744 \\
\hline
\end{tabular}




\begin{tabular}{|c|c|c|}
\hline 25.50 & .795 & .641 \\
\hline 26.50 & .744 & .590 \\
\hline 27.50 & .667 & .538 \\
\hline 28.50 & .590 & .462 \\
\hline 29.50 & .564 & .410 \\
\hline 30.50 & .564 & .359 \\
\hline 31.50 & .538 & .333 \\
\hline 32.50 & .487 & .333 \\
\hline 33.50 & .410 & .282 \\
\hline 35.00 & .359 & .256 \\
\hline 36.50 & .308 & .179 \\
\hline 37.50 & .282 & .154 \\
\hline 38.50 & .256 & .128 \\
\hline 39.50 & .205 & .077 \\
\hline 40.50 & .154 & .077 \\
\hline 41.50 & .128 & .077 \\
\hline 42.50 & .128 & .051 \\
\hline 43.50 & .103 & .051 \\
\hline 44.50 & .103 & .026 \\
\hline 45.50 & .103 & .000 \\
\hline 47.00 & .077 & .000 \\
\hline 49.50 & .051 & .000 \\
\hline 52.00 & .026 & .000 \\
\hline 54.00 & .000 & .000 \\
\hline
\end{tabular}

CLFS- Computerized Lumbar Functional Scale, HC-PAIRS-Health Care Providers' Pain and Impairment Relationship Scale

\section{Multiple Logistic Regression}

\section{Average CLFS Change}

Multiple logistic regression results for CLFS change scores are reported in table 21 . The only significant model that emerged from the data was for predicting a significant improvement in scores in the 5-patient cut-off sample. When controlling the number of patients, the percentage of patients seen by other providers $\left(\beta=.047, S E=.022\right.$, Walds $X^{2}=4.880, p=.034$,

Odds Ratio of 1.045), and having more than 4-years of experience $(\beta=2.650, S E=1.200$, Walds $\mathrm{X}^{2}=4.880, \mathrm{p}=.027$, Odds Ratio of 14.159$)$ were significant predictors. Although a significant 
predictor, the $95 \% \mathrm{Cl}$ interval for the less than 4-years of experience was very wide (.953-

23.165), which calls into question its predictive accuracy. The two-predictor model was significant $(p=.015)$ and had a Nagelkerke pseudo $R^{2}$ of .257 . The overall prediction success rate was $69.4 \%$ with correct prediction rates of $50 \%$ for minimal change and $84.6 \%$ for significant change. Validation of the model with 2017 data found that the coefficients included in the 2018 model were not significant predictors of average number of visits in the 2017 data. When applied to the 2017 data, the model created using the 2018 data had an overall prediction of success of $54.8 \%$.

Table 21. Multiple logistic regression for CLFS change.

\begin{tabular}{|c|c|c|c|c|c|c|}
\hline & \multicolumn{6}{|c|}{$\begin{array}{c}\text { Average CLFS Change Multiple Logistic Regression } \\
\text { Moderate Change 5-Patient Cut-Off Sample }\end{array}$} \\
\hline & \multirow[t]{2}{*}{ Coefficients } & \multirow[t]{2}{*}{$\beta$} & \multirow[t]{2}{*}{ Odds Ratio } & \multicolumn{2}{|c|}{$95 \% \mathrm{Cl}$} & \multirow[t]{2}{*}{$P$} \\
\hline & & & & Lower Bound & Upper Bound & \\
\hline $\begin{array}{l}\text { Model } \\
C=1.547 \\
\text { Pseudo } \\
R^{2}=.090 \\
p=.128\end{array}$ & $\begin{array}{l}\text { Less than } 4 \text { Years of } \\
\text { Experience }\end{array}$ & 1.547 & 4.698 & .953 & 23.165 & .057 \\
\hline \multicolumn{7}{|c|}{ Average CLFS Change Multiple Logistic Regression } \\
\hline $\begin{array}{l}\text { Model } \\
\mathrm{C}=-2.934\end{array}$ & $\begin{array}{l}\text { Less than } 4 \text { Years of } \\
\text { Experience }\end{array}$ & 2.650 & 14.159 & 1.348 & 48.671 & .027 \\
\hline $\begin{array}{l}\text { Pseudo } \\
\mathrm{R}^{2}=.257 \\
\mathrm{P}=.015\end{array}$ & $\begin{array}{l}\text { Percentage of } \\
\text { Patients Seen by } \\
\text { Other Physical } \\
\text { Therapy Providers }\end{array}$ & .047 & 1.048 & 1.004 & 1.094 & .034 \\
\hline \multicolumn{7}{|c|}{ Average CLFS Change Multiple Logistic Regression } \\
\hline $\begin{array}{l}\text { Model } \\
C=-1.339 \\
\text { Pseudo } \\
R^{2}=.067 \\
P=.349\end{array}$ & NPQ Score & .274 & 1.315 & .906 & 1.909 & .149 \\
\hline & \multicolumn{5}{|c|}{ Average CLFS Change Multiple Logistic Regression } & \\
\hline Model & NPQ Score & .072 & 1.074 & .801 & 1.441 & .632 \\
\hline
\end{tabular}




\begin{tabular}{|c|c|c|c|c|c|c|}
\hline $\begin{array}{l}C=1.282 \\
P \text { seudo } \\
R^{2}=.050 \\
P=.192\end{array}$ & & & & & & \\
\hline \multicolumn{7}{|c|}{ Average CLFS Change Multiple Logistic Regression } \\
\hline \multicolumn{7}{|c|}{ Moderate Change 20-Patient Cut-Off Sample } \\
\hline $\begin{array}{l}\text { Model } \\
C=.252 \\
\text { Pseudo } \\
R^{2}=.051 \\
P=.688\end{array}$ & Age 18 to 34 & .841 & 2.319 & .306 & 34.496 & .249 \\
\hline \multicolumn{7}{|c|}{ Average CLFS Change Multiple Logistic Regression } \\
\hline $\begin{array}{l}\text { Model } \\
C=.444 \\
\text { Pseudo } \\
\mathrm{R}^{2}=.099 \\
P=.344\end{array}$ & $\begin{array}{l}\text { Less Than } 4 \text { Years of } \\
\text { Experience }\end{array}$ & 1.649 & 5.200 & .677 & 39.936 & .113 \\
\hline
\end{tabular}

CLFS- Computerized Lumbar Functional Scale, NPQ- Neurophysiology of Pain Questionnaire, C-Constant, AR ${ }^{2}$-Adjusted R²

\section{Average CLFS Residual}

Multiple logistic regression results for CLFS residual scores are reported in Table 22.

Multiple logistic regression for CLFS residual scores produced significant models for the 5patient and 10-patient cut off samples. When controlling for the number of patients, a fourpredictor model significantly predicted CLFS residual categories $(p=.002)$ with a Nagelkerke pseudo $R^{2}$ of .265 . The model included HC-PAIRS scores $\left(\beta=.093, S E=.038\right.$, Walds $X^{2}=5.868, p=$ .015 , Odds Ratio of 1.097), average intake FABQpa $\left(\beta=-.083, S E=.036\right.$, Walds $X^{2}=5.219, p=.022$, Odds Ratio of .920), average FABQpa change $\left(\beta=-.082, S E=.033\right.$, Walds $X^{2}=6.075, p=.014$, Odds Ratio of .921) and physical therapists 55 years or older $\left(\beta=-1.538, S E=.729\right.$, Walds $X^{2}=4.451, p=$ .035 , Odds Ratio of .215). The overall prediction success rate was $70.9 \%$ with correct prediction rates of $50 \%$ for a less than expected change and $84.6 \%$ for more than predicted change. Validation of the model with 2017 data found that average FABQpa intake and change were the only significant predictors of CLFS residual categories in the 2017 data. When applied to the 
2017 data, the model created using the 2018 data had an overall prediction of success of $68.2 \%$.

A four-predictor model also significantly predicted CLFS residual scores $(p=.001)$ for the 10patient cut off sample with Nagelkerke pseudo $R^{2}$ of .324 . This model contained three of the same predictors as the 5 -patient cut-off sample, HC-PAIRS $\left(\beta=.115, \mathrm{SE}=.043\right.$, Walds $X^{2}=7.078$, $p=.008$, Odds Ratio of 1.122), average intake FABQpa $\left(\beta=-.111, S E=.040\right.$, Walds $X^{2}=7.576, p=$ .006 , Odds Ratio of .895$)$ and average FABQpa change $\left(\beta=-.101, S E=.040\right.$, Walds $X^{2}=3.988, p=$ .046 , Odds Ratio of .904). The final predictor was a physical therapist with 5-9 years of experience $\left(\beta=1.366, \mathrm{SE}=.684\right.$, Walds $X^{2}=3.988, p=.046$, Odds Ratio of 3.921$)$ and was unique to the 10-patient cut-off sample. The overall prediction success rate was $76 \%$ with correction prediction rates of $58.6 \%$ for less than expected change and $87 \%$ for more than expected change. Validation of the model with 2017 data found that average FABQpa intake and change were the only significant predictors of CLFS residual categories in the 2017 data. When applied to the 2017 data, the model created using the 2018 data had an overall prediction of success of $52.7 \%$.

Table 22. Multiple logistic regression for average CLFS residual

\begin{tabular}{|c|c|c|c|c|c|c|}
\hline & \multicolumn{6}{|c|}{$\begin{array}{c}\text { Average CLFS Residual Multiple Logistic Regression } \\
\text { 5-Patient Cut-Off Sample }\end{array}$} \\
\hline & \multirow[t]{2}{*}{ Coefficients } & \multirow[t]{2}{*}{$\beta$} & \multirow[t]{2}{*}{ Odds Ratio } & \multicolumn{2}{|c|}{$95 \% \mathrm{Cl}$} & \multirow[t]{2}{*}{$P$} \\
\hline & & & & Lower Bound & Upper Bound & \\
\hline \multirow{4}{*}{$\begin{array}{l}\text { Model } \\
C=.777 \\
\text { Pseudo } \\
R^{2}=.265 \\
p=.002\end{array}$} & HC-PAIRS & .093 & 1.097 & 1.018 & 1.182 & .015 \\
\hline & Average FABQpa Intake & -.083 & .920 & .857 & .988 & .022 \\
\hline & Average FABQpa Change & -.082 & .921 & .863 & .983 & .014 \\
\hline & $\begin{array}{l}55 \text { Years of Age or } \\
\text { Older }\end{array}$ & -1.538 & .215 & .051 & .897 & .035 \\
\hline
\end{tabular}




\begin{tabular}{|c|c|c|c|c|c|c|}
\hline \multicolumn{7}{|c|}{$\begin{array}{c}\text { Average CLFS Residual Multiple Logistic Regression } \\
\text { 10-Patient Cut-Off Sample }\end{array}$} \\
\hline \multirow{4}{*}{$\begin{array}{l}\text { Model } \\
C=.621 \\
\text { Pseudo } \\
R^{2}=.324 \\
P=.001\end{array}$} & HC-PAIRS & .115 & 1.122 & 1.031 & 1.222 & .008 \\
\hline & Average FABQpa Intake & -.111 & .895 & .826 & .968 & .006 \\
\hline & Average FABQpa Change & -.101 & .904 & .836 & .976 & .010 \\
\hline & $\begin{array}{l}5-9 \text { Years of } \\
\text { Experience }\end{array}$ & 1.366 & 3.921 & 1.026 & 14.991 & .046 \\
\hline \multicolumn{7}{|c|}{ Average CLFS Residual Multiple Logistic Regression } \\
\hline $\begin{array}{l}\text { Model } \\
C=-.678 \\
\text { Pseudo } \\
R^{2}=.126 \\
P=.080\end{array}$ & Average FABQpa Change & -.097 & .908 & .825 & .999 & .047 \\
\hline
\end{tabular}

FABQpa- Fear Avoidance Beliefs Questionnaire physical activity subscale, HC-PAIRS-Health Care Providers' Pain and Impairment Relationship Scale, C-Constant, $\mathrm{AR}^{2}$-Adjusted $\mathrm{R}^{2}$

\section{Summary}

Data analysis to predict influences on attitudes and beliefs was performed on data from 140 physical therapists. Analysis to predict factors that influenced patient outcomes was performed on three samples that were based on the number of patients seen by a therapist.

The 5-patient cut-off sample included 92 physical therapists and 2,448 patients. The 10-patient cut-off sample included 78 physical therapists and 2,345 patients. The 10-patient cut-off sample included 54 physical therapists and 2,016 patients.

Simple linear regression showed that physical therapist HC-PAIRS scores were predicted by entry-level degree, board certification, practice setting, percentage of LBP-related caseload, and NPQ scores. PABS-PT scores were predicted by the physical therapists age, level of experience, entry-level degree, residency and fellowship training, board certification, practice setting, and NPQ scores. Model building using multiple linear regression led to a one-predictor model for HC-PAIRS that only included NPQ scores. Higher NPQ scores were associated with 
lower HC-PAIRS scores and the model explained about $15 \%$ of the variability. PABS-BM scores were predicted by a physical therapist age between 18-34, board certification, and NPQ scores. Lower PABS-BM scores were associated with having an age between 18-34, being a board certified clinical specialist, and higher NPQ scores. This model explained $28 \%$ of the variability in PABS-BM scores. PABS-BPS scores were also predicted by a physical therapist age between 1834, board certification and NPQ scores. Higher PABS-BPS scores were associated with having an age between 18-34, being board certified and higher NPQ scores. This model predicted about $23 \%$ of the variability in PABS-BPS scores.

Simple linear regression showed that average CLFS change scores were predicted by intake FABQ scores, changes in FABQ scores, HC-PAIRS scores, and PABS-BM scores. The only predictor that was significant across all three cut-off samples was a change in FABQ scores. Average CLFS residual scores were predicted by changes in FABQ scores, HC-PAIRS scores, and level of experience. The only predictor that was significant across all three cut-off samples was a change in FABQ scores. Average number of patient visits was predicted by intake FABQ scores, HC-PAIRS scores, the physical therapists' age, and level of experience. There were no consistent univariate predictors of the number of patient visits across the three samples.

Linear predictive models were created using a backwards stepwise approach for CLFS change, CLFS residual and the average number of visits. Only two of the models included the attitudes and beliefs of physical therapists. For the 10-patient cut-off sample, greater improvement in CLFS were predicted by higher HC-PAIRS scores and reductions in FABQ scores. This model explained approximately $19 \%$ of the variability in CLFS scores. Higher average number of visits was predicted by greater HC-PAIRs scores and a greater reduction in FABQ for 
the 10 -patient cut-off sample. This model predicted nearly $13 \%$ of the variable in the number of visits.

Simple logistic regression found that having 4 years of experience or less, and the percentage of care provided by other physical therapy providers were the only two univariate predictors of CLFS change scores at the 5-patient cut-off. Having a physical therapist with 4 years of experience or less was the only predictor of CLFS change for the 10-patient and 20patient cut-off samples. The only univariate predictor of CLFS residual scores was HC-PAIRS scores for the 10-patient cut-off sample. An ROC curve for HC-PAIRS predicting CLFS change scores had an AUC of .620 and a cut-off to maximize specificity (.641) and sensitivity (.546) of 30.50 .

Logistic predictive models were created using a backwards stepwise approach for CLFS change and CLFS residual. Physical therapist beliefs were included in the predictive model for CLFS residual for the 5-patient and 10-patient cut-off samples. A higher than predicted residual score was predicted by higher HC-PAIRS scores, lower intake FABQ scores, a reduction in FABQ scores, and being treated by a physical therapist 55 years or older for the 5 -patient cut-off sample. This model explained approximately $26 \%$ of the variability in CLFS residual classification. Higher HC-PAIRS scores, lower FABQ intake scores, a reduction in FABQ scores, and 5-9 years of experience were predictive of a higher than predicted residual score for the 10-patient cut-off sample. This explained about $32 \%$ of the variability in CLFS residual classification. 


\section{Chapter 5: Discussion}

\section{$\underline{\text { Introduction }}$}

This chapter will discuss the findings of the project. It will start by covering the LBPrelated attitudes and beliefs of physical therapists and the factors that influence those beliefs. Next will be a discussion of the impact of attitudes and beliefs on the outcomes of patients. This will be followed by sections on the implications of the findings, limitations and delimitations, and the author's recommendations. The chapter will end with a summary that reviews the content of the chapter.

\section{Discussion}

\section{Physical Therapist Low Back Pain Related Attitudes and Beliefs}

One of the main goals of this dissertation was to explore the low back pain (LBP) related attitudes and beliefs of physical therapists in the United State (US). In this dissertation, the average score on the Health Care Providers' Pain and Impairment Relationship Scale (HC-PAIRS) was 33.34, and Pain Attitudes and Beliefs Scale for Physiotherapists (PABS-PT) average subscale scores were 26.56 for the biomedical subscale (PABS-BM) and 36.47 for the biopsychosocial subscale (PABS-BPS). Previous studies have shown that higher HC-PAIRS and PABS-BM scores, and lower PABS-BPS scores may have a negative impact on clinicians' clinical behaviors ${ }^{46,48,63}$ and patient beliefs. ${ }^{155}$ Although studies have looked at the influence of beliefs on clinical behaviors, there are no established cut-off points for HC-PAIRS or PABS-PT scores. In the literature, HC-PAIRS scores have been reported as high as 57.9 in 72 Irish and United Kingdom based student physical therapists ${ }^{187}$ and as low as 28.3 in 12 physical therapists from the United States. ${ }^{189}$ PABS-BM scores have ranged from 26.7 in 12 US based physical therapists ${ }^{189}$ to 41.4 
in 42 physical therapists from Sweden. ${ }^{44}$ The same 42 Swedish physical therapists had the lowest reported PABS-BPS scores (25.9) ${ }^{44}$ and the highest scores have been reported in 42 physical therapists from the Netherlands (40.4). ${ }^{165}$ The HC-PAIRS and PABS-BPS scores for the participants in this dissertation fell well within the reported scores from other studies. Scores on the PABS-BM subscale were on the low end of the reported values and were similar to a study that also included US based physical therapists. It is possible that US based physical therapists have less biomechanically-oriented attitudes and beliefs than therapists in other countries; however, there is only one other study using the 19-item PABS-PT scale in US based physical therapists and it only included 12 physical therapists. ${ }^{189}$

Several factors predicted the LBP-related attitudes and beliefs of physical therapists who participated in this study. The largest and most consistent predictor was physical therapist knowledge of pain neuroscience. Higher scores on the Neurophysiology of Pain Questionnaire (NPQ) were predictive of beliefs that were less biomedically-oriented and more biopsychosocially-oriented. This is not surprising given that the current science on pain suggests that pain is multifactorial and includes biomedical, psychological, and social factors. ${ }^{229-231}$ The NPQ asks questions that are designed to test if respondents understand the complex, multifaceted nature of pain. Physical therapists with greater knowledge about this multifactorial nature will likely have attitudes and beliefs that align with this knowledge. The potential connection between knowledge and attitudes and beliefs was also supported by the predictive ability of entry-level degree, residency and fellowship training, and certifications. Physical therapists with an entry-level Doctorate degree, residency or fellowship training, and/or board certification were more likely to have biopsychosocially-oriented beliefs. This 
relationship may be at least partially explained by the impact education and certification have on NPQ scores. Fellowship (10.42) and residency trained (10.11) physical therapists had some of the highest NPQ scores and individuals with an entry-level Doctorate degree also had high NPQ scores (9.89). Physical therapists with an entry-level Doctorate degree $(p=.010)$, board certification $(p=.005)$ and who were fellowship trained $(p=.030)$ had statistically significantly higher NPQ scores than physical therapists without the same training or certification. NPQ scores were higher for residency-trained physical therapists, but the difference was not statistically significant.

Physical therapist age, years of experience, caseload of LBP patients, and practice setting predicted between 1.6 and $5.6 \%$ of the variability in LBP-related attitudes and beliefs. Younger professionals (18-34 years) with less years of experience (5-9 years) had more biopsychosocially-oriented beliefs than older ( $\geq 55$ years), more experienced physical therapists ( $\geq 25$ years). This finding is consistent with studies from Fullen et al., ${ }^{63}$ Epstein-Sher et al., ${ }^{143}$ and Magalhaes et al. ${ }^{171}$ that found physicians and physical therapists with less experience had lower scores on the PABS-BM. The trend towards more biomedically-oriented attitudes and beliefs in older more experienced clinicians may be related to clinical experience and/or education. It is possible that clinical experience reinforces stronger biomedically-oriented beliefs. Clinicians with more experience treating patients with bio-pathoanatomical (BPA) issues may develop beliefs that these BPA factors play a major role in the development and recovery from painful conditions. It is also possible that trends in education have changed and older, more experienced therapists have had less educational exposure to modern pain science and biopsychosocial concepts. 
It was hypothesized that a personal history of LBP might have an influence on LBPrelated attitudes and beliefs. This hypothesis was reasonable given the evidence that patients' personal experience has an influence on their pain-related beliefs. ${ }^{57,58} \mathrm{~A}$ qualitative study by Askew et al. also found that physical therapists believed that their personal experience with pain had an impact on their attitude towards patients. ${ }^{145}$ On the other hand, several quantitative studies have found that a personal history of LBP does not have an influence on the attitudes and beliefs of clinicians. ${ }^{142,172,173}$ For example, Coudeyre et al found that a personal history of LBP did not influence the fear avoidance beliefs of French physicians. In this dissertation, the influence of LBP on the attitudes and beliefs of physical therapists was investigated in more detail than previous quantitative studies. Therapists were asked if they had an experience with LBP and the level of impact that pain had on their lives. It was hypothesized that LBP significantly affecting a physical therapist's life would have a more substantial influence on their attitudes and beliefs about LBP. In this study, $96.3 \%$ of the physical therapists had a history of LBP. Of those with a history of LBP, $58.9 \%$ (76) were minimally impacted by the pain and $10.1 \%$ (13) reported that LBP had a significant impact on their life. Despite taking a more nuanced look at the impact of LBP, it was still not a significant predictor of LBP-related attitudes and beliefs. It seems that, even though physical therapists perceive that their personal experience with pain has an impact on their perception of patients, it doesn't have an appreciable influence on their LBP-related attitudes and beliefs.

Multiple regression analysis produced predictive models for both PABS-BM and PABS-BPS. When building a model for HC-PAIRS, NPQ score was the only significant predictor. A model containing NPQ scores, board certification, and an age between 18-34 predicted $27.8 \%$ 
of the variability in PABS-BM and $23.3 \%$ in PABS-BPS. The strongest predictor of PABS-BM scores was NPQ scores (standardized $\beta=-.421$ ) and the strongest predictor of PABS-BPS was board certification (standardized $\beta=-.269$ ), followed closely by NPQ scores (standardized $\beta=-$ .255). This suggests that LBP attitudes and beliefs may be modifiable through education. Gaining an increased understanding of current pain science and obtaining the skills to become board certified may lead to LBP-related attitudes and beliefs that are more aligned with the biopsychosocial model. This is supported by several studies that have shown education, including pain science and biopsychosocial principles, leads to changes in LBP-related attitudes and beliefs. ${ }^{44,125,141,165,166,180,185,187-189,232-235}$ For example, Colleary et al. looked at changes in NPQ and HC-PAIRS scores after providing education on pain science or a control education intervention that included red flags, Waddell's signs, and detecting tissue pathology. ${ }^{187}$ The intervention group had significant changes in NPQ scores and HC-PAIRS scores, in the expected direction. The intervention group was also more likely to provide advice to return to work, exercise, stay active, and avoid bed rest. One study was found that showed no change in attitudes and beliefs after a 3-hour education session on pain science. Student physical therapists' NPQ and HC-PAIRS scores were measured at baseline, immediately after the education, and at 6 months. NPQ scores increased after the education, but there was no significant difference in HC-PAIRS at any time point. It is possible that one 3-hour session was not sufficient to cause a significant change in beliefs. The other studies that showed changes in attitudes and beliefs after education either included more than one session ${ }^{44,163,165,232}$ or had one session that was longer than 3 hours. ${ }^{164,233-235}$ 


\section{Impact of Beliefs on Outcomes}

The other main objective of this dissertation was to investigate the impact of physical therapist LBP-related attitudes and beliefs on the outcomes of patients. To do this, the attitudes and beliefs data collected by the physical therapist survey was compared to the average Computerized Lumbar Functional Scale (CLFS) scores, average CLFS residual scores, and the average number of visits collected from Focus on Therapeutic Outcomes, Inc. (FOTO). The PABS-BPS subscales were not predictive of outcomes and the PABS-BM subscale was only predictive of average CLFS change for the 10-patient cut-off sample. HC-PAIRS score was a univariate linear predictor of average CLFS score, average CLFS residual score, and average number of visits in the 10-patient cut-off sample. When controlling for the number of patients seen, a linear model containing HC-PAIRS scores and the average change in the Fear Avoidance Beliefs Questionnaire (FABQ) predicted $16.1 \%$ of the variability in average CLFS change scores and $12.8 \%$ of the variability in the average number of visits. HC-PAIRS was also a univariate logistic predictor of average CLFS residual for the 10-patient cut-off sample. Receiver operating characteristic (ROC) curve identified a cut-off score of 30.50. This score had weak discriminatory value with a sensitivity of .564 and specificity of .641 .

HC-PAIRS was a predictor of some patient outcomes in this study; however, the direction of the prediction was not in the expected directions. Physical therapists with higher HC-PAIRS scores, indicating a stronger belief that pain is associated with disability, had greater average CLFS change, greater average CLFS residual scores, and saw patients for fewer visits. Based on this, it is possible that physical therapists with beliefs that associate pain and disability act in a way that improves outcomes. Several studies have shown that higher HC-PAIRS scores 
are associated with certain clinician behaviors. ${ }^{46,54,138,148}$ For example, Rainville et al. studied 82 physicians and found that higher HC-PAIRS scores were correlated with advice to avoid activity and stay out of work. ${ }^{54}$ Houben et al. also found that physical therapist students with higher HC-PAIRS scores provided advice and recommended treatments that were more biomedicallyoriented. ${ }^{138}$ For example, students who scored higher on the HC-PAIRS were less likely to suggest that increasing exercise is safe, even if there is some pain. It has been suggested that the psychological- and behavioral-oriented treatment strategies seen in these studies are more effective than pure biomedical approaches. ${ }^{29,85,236}$ Although the evidence for this is growing, there are some who caution that we may be moving too far from the biological part of the biopsychosocial model. ${ }^{237}$ It is possible that physical therapists with beliefs that are too focused on psychological and social factors miss important biomedical components of LBP, leading to worse outcomes.

It is also possible that the predictive value of HC-PAIRs was a statistical anomaly in this dataset and is not generalizable outside of this sample. In this dissertation, the relationship between HC-PAIRS and outcomes was tested 33 times. Out of those 33 tests, HC-PAIRS was a significant predictor eight times (24.2\%). Cross validation of the multiple regression findings using 2017 data from the same sample of physical therapists found that only one out of the four models including HC-PAIRS could be validated. The 10-patient sample CLFS change model, which included HC-PAIRS, was validated in the 2017 sample; however, the residual correlation was poor (.238), indicating low predictive value. Since this study was exploratory in nature, statistical safe guards for $p$-value inflation were not used. As a result, the significant findings for HC-PAIRS scores may have been influence by alpha inflation. If the alpha used in this 
dissertation was adjusted for multiple comparisons ( $\alpha=.002, .05 / 33)$ HC-PAIRS would no longer be a significant predictor for any of the outcome measures.

As discussed in Chapter 2 of this dissertation, there is theoretical support for a connection between biopsychosocial-oriented beliefs and improved patient outcomes. These beliefs seem to influence clinician behaviors, ${ }^{54,138,152}$ patient beliefs, ${ }^{47,56,144}$ and clinician expectations of recovery and disability ${ }^{66,157}$ in a way that will theoretically promote better outcomes. Despite this theoretical support, the results of this dissertation seem to align with other studies that have shown no connection between biopsychosocially-oriented attitudes and beliefs and outcomes. ${ }^{144,148,163}$ It is possible that physical therapist LBP-related attitudes and beliefs have a small influence on outcomes, but this influence is hidden by the many other factors that can influence patient recover. It is also possible that physical therapist attitudes and beliefs have an impact on outcomes in a subset of patients. For example, the outcomes of patients with chronic LBP, or patients at risk for developing chronic LBP, may be more likely influenced by psychologically informed interventions. ${ }^{133,134}$ It is also possible that patients with more extreme psychosocial influences, such as fear avoidance or catastrophizing, have greater sensitivity to the attitudes and beliefs of physical therapist. The data in this dissertation did not allow for subset analysis based on patient characteristics, so it is unclear if there is a subset of patients whose outcomes are influenced by clinician beliefs.

The most consistent predictor of outcomes in this dataset was changes in FABQ physical activity (FABQpa) scores. Change in average FABQpa score was a very consistent linear predictor for all outcomes except number of visits. It was not a univariate logistic predictor of any outcome, but was a multivariate predictor of average CLFS residual change categories. 
There was a fairly large change in the strength of FABQpa prediction across the patient cut-off samples. For average CLFS change scores, FABQ change predicted $9.5 \%$ of the variance for the 5-patient cut-off sample, $10 \%$ in the 10 -patient cut-off, and $20.6 \%$ in the 20 -patient cut-off. This trend was also seen for CLFS residual with slightly lower predictive ability $(7.2 \%, 8.2 \%, 18.5 \%)$. This relationship between FABQ scores and outcomes is consistent with previous work that has found an association between improved disability and reduction in FABQ scores. ${ }^{36,59,238,239}$ The direction of causality in this relationship is still unclear and it is possible that improved disability leads to reduction in fear avoidance. Studies that have found a relationship between baseline FABQ scores and pain, disability, and return to work suggest that FABQ scores may be a mediator of improved outcomes; however, intake FABQpa scores in this dissertation were not consistently associated with outcomes. For example, intake FABQpa was a linear predictor of average CLFS change and number of visits only in the 20-patient cut-off sample. If FABQpa scores do mediate changes in outcomes, it may be important for clinicians to focus interventions on reducing fear avoidance in patients. ${ }^{8}$ Clinicians with more biopsychosociallyoriented beliefs may be more likely to provide interventions that target a reduction in fear avoidance.

\section{$\underline{\text { Implications }}$}

This dissertation is one of the first studies to investigate the impact of LBP-related attitudes and beliefs of physical therapists. These attitudes and beliefs are closely related to pain science knowledge, which implies that educational interventions aimed at improving knowledge are likely to increase the biopsychosocial orientation of attitudes and beliefs. Despite the apparent ability to influence the beliefs of physical therapists, this study questions 
the usefulness of efforts to change those beliefs. Physical therapist LBP-related attitudes and beliefs were not consistent predictors of patient outcomes. This suggests that although LBPrelated attitudes and beliefs appear to be modifiable, there is no evidence that physical therapists with more biopsychosocial-oriented beliefs have better outcomes. Instead, the results of this dissertation suggest that physical therapists who associate pain with disability may have improved outcomes. As a result, if improving outcomes is the goal, this dissertation indicates that educational interventions aimed at increasing the biopsychosocial orientations of physical therapists may not be an effective use of resources.

\section{Limitations and Delimitations}

Several limitations and delimitations should be considered when interpreting the results of this study. These limitations and delimitations will be discussed in two categories. The first category is limitations and delimitations regarding the physical therapists used in this dissertation. The physical therapists in this dissertation were users of the FOTO outcomes registry. This is a commercial outcomes service that is used by only a small number of physical therapists in the US. Physical therapists who use the FOTO system may not accurately represent US physical therapists. The obtained sample was one of convenience and only represented $0.6 \%$ of FOTO users. As a result, it is possible that the sample was biased and cannot be generalized to other FOTO users or to US physical therapists who are not FOTO users.

In this dissertation, physical therapist attitudes and beliefs were measured prospectively, but outcomes data was retrospective. As a result, it is unclear what the physical therapists' beliefs were when they were treating the patients included in this dissertation. To explore this potential limitation, survey respondents were asked if their beliefs had changed 
over the past year. Approximately $81 \%$ of the respondents indicated that they had experienced minimal to no change in their beliefs over the past year. Pilot testing also suggested that scores on the HC-PAIRS and PABS-PT were stable over a 1-year period. There was no difference in outcomes based on change in beliefs and change in beliefs did not have a significant impact on the predictive models.

There was also no way to control for who provided the physical therapy services. In the FOTO system, patients are assigned to a provider based on who performs the initial evaluation. It is possible that other physical therapy providers delivered care for a patient who was assigned to a different physical therapist in the FOTO system. To mitigate this limitation, physical therapists were asked to identify the percentage of their patients seen by other physical therapy providers. This variable was used in regression modeling when it significantly influenced the model.

The second category of limitations and delimitations pertains to the patient data. The patient data only included patients who had complete CLFS scores at the initial evaluation and discharge. It is unclear what percentage of patients seen by each physical therapist were included in this dataset. It is possible that a significant number of patients were eliminated because they did not have a discharge CLFS score. There may have been non-random factors that influenced which patients completed the discharge CLFS. For example, patients who were not responding well to treatment may have been more likely to stop treatment sessions before a follow-up or discharge CLFS score was obtained. This could bias the sample, leaving a higher percentage of improved patients in the dataset. 
FOTO only provided mean patient data for each participating physical therapist. This limited the analysis that could be performed on the data. For example, specific patient characteristics could not be controlled for in the modeling of raw CLFS scores and average number of visits. The lack of individualized data also prevented sub-group analysis based on factors such as chronicity and FABQpa scores. Only having mean data also reduced the ability to screen the patient data for outliers. Outlier analysis could only be performed on the means provided by FOTO. This may have been particularly problematic for physical therapists with only a small number of completed patient cases. To address this, three samples were analyzed based on the number of completed cases.

\section{$\underline{\text { Recommendations }}$}

Based on the results of this dissertation, the attitudes and beliefs of physical therapists do not appear to have a major impact on the outcomes of patients. As a result, if improving outcomes is the goal, there is no support for spending time and resources in an attempt to influence the attitudes and beliefs of physical therapists. Educating physical therapists on the most current pain neuroscience may still be useful, but it is unlikely that changes in LBP-related attitudes and beliefs due to education will have a significant influence on patient outcomes.

Future studies should take two broad approaches. One approach should be replication studies that utilize large datasets. It is possible that the results of this dissertation are biased due to the limitations of the data. Replication studies that utilize a more representative population of physical therapists, include raw patient data, and contain more information about dropouts may produce different results. The other suggested approach is to perform prospective studies with methodology that controls for potential confounding variables. For 
example, these studies could better control for changes in physical therapist beliefs, the influence of other physical therapy providers, the impact of dropouts, and they could better assess the influence of attitudes and beliefs on the clinical behaviors of the treating physical therapists.

\section{Summary}

The LBP-related attitudes and beliefs of physical therapists in the US are similar to those in other countries. The average PABS-BM score for participants in this dissertation was towards the low end of the reported values, suggesting that the physical therapists in this sample had attitudes and beliefs that were less biomedically-oriented. Several factors predicted LBP-related attitudes and beliefs, with the most consistent predictor being knowledge of current pain science. The LBP-related attitudes and beliefs of physical therapists were not consistent predictors of outcomes. HC-PAIRS scores were found to be related to outcomes in 8 of the 33 performed analyses; however, this relationship was not in the predicted direction. Physical therapists who believed there was a strong relationship between pain and disability had improved outcomes. Limitations of this dissertation include a potentially biased sample of physical therapists, an inability to control certain variables, and limited information about individual subjects. Future studies should attempt to replicate these findings. In addition, prospective studies with better controls should be performed. 


\section{Appendix}

\section{Demographic Survey}

\begin{tabular}{|c|c|c|}
\hline $\begin{array}{l}\text { Select the answer that best describes your current } \\
\text { role in the field of physical therapy. }\end{array}$ & $\begin{array}{l}\circ \\
\circ \\
\circ\end{array}$ & $\begin{array}{l}\text { Physical therapist } \\
\text { Physical therapist assistant } \\
\text { Other }\end{array}$ \\
\hline What is your current age? & $\begin{array}{l}0 \\
0 \\
0 \\
0 \\
0 \\
0 \\
0 \\
0\end{array}$ & $\begin{array}{l}18-24 \\
25-34 \\
35-44 \\
45-54 \\
55-64 \\
65-75 \\
75 \text { years or older } \\
\end{array}$ \\
\hline What gender identity best describes you? & $\begin{array}{l} \\
\circ \\
0 \\
0 \\
0 \\
0\end{array}$ & $\begin{array}{l}\text { Male } \\
\text { Female } \\
\text { Transgender Male } \\
\text { Transgender Female } \\
\text { Other } \\
\text { Prefer Not to Answer }\end{array}$ \\
\hline Which ethnicity/race best describes you? & $\begin{array}{l}\circ \\
\circ \\
\circ \\
\circ\end{array}$ & $\begin{array}{l}\text { White, Non-Hispanic } \\
\text { Hispanic or Latino } \\
\text { Black or African American, Non- } \\
\text { Hispanic } \\
\text { Native American } \\
\text { Asian or Pacific Islander } \\
\text { Other } \\
\text { Prefer Not to Answer }\end{array}$ \\
\hline $\begin{array}{l}\text { How many years have you been a licensed physical } \\
\text { therapist or physical therapist assistant? }\end{array}$ & $\begin{array}{l} \\
0 \\
0 \\
0 \\
0 \\
0\end{array}$ & $\begin{array}{l}\text { Less than } 4 \text { years } \\
5-9 \\
10-14 \\
15-19 \\
20-24 \\
25 \text { years or greater } \\
\end{array}$ \\
\hline What was your entry-level degree? & $\begin{array}{l} \\
\circ \\
\circ\end{array}$ & $\begin{array}{l}\text { Bachelor's (e.g. BSPT) } \\
\text { Master's (e.g. MPT) } \\
\text { Doctorate (e.g. DPT) }\end{array}$ \\
\hline $\begin{array}{l}\text { Please indicate if you have completed any of the } \\
\text { following post-graduate programs. (check all that } \\
\text { apply) }\end{array}$ & $\begin{array}{l}0 \\
0 \\
0 \\
0 \\
0 \\
0 \\
0 \\
0\end{array}$ & $\begin{array}{l}\text { None } \\
\text { tDPT } \\
\text { PhD } \\
\text { DsC } \\
\text { Residency } \\
\text { Fellowship } \\
\text { Other }\end{array}$ \\
\hline $\begin{array}{l}\text { Please indicate if you have obtained any of the } \\
\text { following certifications. (check all that apply) }\end{array}$ & $\begin{array}{l}0 \\
0 \\
0 \\
0\end{array}$ & $\begin{array}{l}\text { None } \\
\text { Board Certification in Orthopedics } \\
\text { Board Certification in Sports } \\
\text { Board Certification in Geriatrics }\end{array}$ \\
\hline
\end{tabular}




\begin{tabular}{|c|c|c|}
\hline & $\begin{array}{l}\circ \\
\circ\end{array}$ & $\begin{array}{l}\text { Manual Therapy Certification } \\
\text { Other }\end{array}$ \\
\hline $\begin{array}{l}\text { What setting(s) do you currently practice in? (check all } \\
\text { that apply) }\end{array}$ & $\begin{array}{l}\circ \\
\circ \\
\circ\end{array}$ & $\begin{array}{l}\text { Private Practice Outpatient } \\
\text { Orthopedics } \\
\text { Hospital-Based Outpatient } \\
\text { Orthopedics } \\
\text { Rehabilitation Facility Based } \\
\text { Outpatient Orthopedics } \\
\text { Other Outpatient Setting } \\
\text { Inpatient } \\
\text { Other }\end{array}$ \\
\hline $\begin{array}{l}\text { What percentage of your overall caseload consists of } \\
\text { patients with low back pain as their primary complaint? }\end{array}$ & $\begin{array}{l}0 \\
0 \\
0 \\
0 \\
0\end{array}$ & $\begin{array}{l}\text { None } \\
\text { Less than } 10 \% \\
10-24 \% \\
25-50 \% \\
\text { Greater than } 50 \%\end{array}$ \\
\hline $\begin{array}{l}\text { Approximately what percentage of your patients with } \\
\text { low back pain are seen by other physical therapy } \\
\text { providers within your clinic (i.e. other physical } \\
\text { therapists or assistants) }\end{array}$ & & 0-------------------------100\% \\
\hline $\begin{array}{l}\text { Of those patients seen by other physical therapy } \\
\text { providers in your clinic, what is the usual percentage of } \\
\text { care provided by you? }\end{array}$ & & 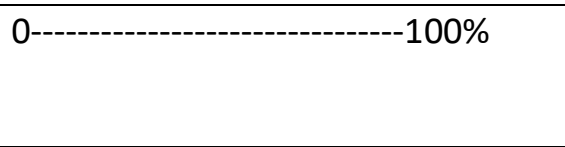 \\
\hline Have you ever experienced low back pain? & $\begin{array}{l}0 \\
\circ\end{array}$ & $\begin{array}{l}\text { Yes } \\
\text { No }\end{array}$ \\
\hline How large of an impact has back pain had on your life? & $\begin{array}{l} \\
\circ \\
\circ\end{array}$ & $\begin{array}{l}\text { None } \\
\text { Minimal } \\
\text { Significant }\end{array}$ \\
\hline On a typical week, how often do you exercise & $\begin{array}{l}0 \\
0 \\
0 \\
0 \\
0 \\
0 \\
0 \\
0\end{array}$ & $\begin{array}{l}\text { Not at all } \\
1 \text { time a week } \\
2 \text { times a week } \\
3 \text { times a week } \\
4 \text { times a week } \\
5 \text { times a week } \\
\text { More than } 5 \text { times a week }\end{array}$ \\
\hline $\begin{array}{l}\text { On a typical week, what type of exercise do you } \\
\text { perform? (Check all that apply) }\end{array}$ & $\begin{array}{l}\circ \\
\circ \\
\circ \\
\circ\end{array}$ & $\begin{array}{l}\text { Cardiovascular exercises (e.g. } \\
\text { running, walking, biking) } \\
\text { Machine based strength training } \\
\text { Free weight based strength training } \\
\text { Body weight exercises } \\
\text { High intensity interval training }\end{array}$ \\
\hline $\begin{array}{l}\text { How have your attitudes and beliefs about back pain } \\
\text { changed in the past year? }\end{array}$ & $\begin{array}{l}0 \\
0 \\
0\end{array}$ & $\begin{array}{l}\text { There has been no change } \\
\text { There has been minimal change } \\
\text { There has been a significant change }\end{array}$ \\
\hline $\begin{array}{l}\text { What factor(s) influenced your change in attitudes and } \\
\text { beliefs? (check all that apply) }\end{array}$ & $\begin{array}{l}0 \\
\circ \\
0\end{array}$ & $\begin{array}{l}\text { A book(s) } \\
\text { A research article(s) } \\
\text { A colleague(s) }\end{array}$ \\
\hline
\end{tabular}




\begin{tabular}{|l|l|}
\hline & $\circ$ A course(s) \\
& $\circ$ Other \\
\hline
\end{tabular}


Health Care Providers' Pain and Impairment Relationship Scale (HC-PAIRS), 13-item Version Instructions: Please respond by selecting the response that most closely describes the way you feel about each of the following statements.

1. Chronic back pain patients can still be expected to fulfill work and family responsibilities, despite pain.

\begin{tabular}{|l|l|l|l|l|l|l|}
\hline $\begin{array}{l}\square \\
\text { Completely } \\
\text { Disagree }\end{array}$ & $\begin{array}{l}\square \\
\text { Disagree }\end{array}$ & $\begin{array}{l}\square \\
\text { Disagree } \\
\text { Somewhat }\end{array}$ & $\begin{array}{l}\square \\
\text { Neutral }\end{array}$ & $\begin{array}{l}\square \\
\text { Agree } \\
\text { Somewhat }\end{array}$ & $\begin{array}{l}\square \\
\text { Agree }\end{array}$ & $\begin{array}{l}\square \\
\text { Completely } \\
\text { Agree }\end{array}$ \\
\hline
\end{tabular}

2. An increase in pain is an indicator that a chronic back pain patient should stop what he/she is doing until the pain decreases.

\begin{tabular}{|l|l|l|l|l|l|l|}
\hline$\square$ & $\square$ & $\square$ & $\square$ & $\square$ & $\square$ & $\square$ \\
$\begin{array}{l}\text { Completely } \\
\text { Disagree }\end{array}$ & Disagree & $\begin{array}{l}\text { Disagree } \\
\text { Somewhat }\end{array}$ & Neutral & $\begin{array}{l}\text { Agree } \\
\text { Somewhat }\end{array}$ & $\begin{array}{l}\text { Agree } \\
\text { Completely } \\
\text { Agree }\end{array}$ \\
\hline
\end{tabular}

3. Chronic back pain patients cannot go about normal life activities when they are in pain.

\begin{tabular}{|l|l|l|l|l|l|l|}
\hline $\begin{array}{l}\text { Completely } \\
\text { Disagree }\end{array}$ & $\begin{array}{l}\text { Disagree } \\
\text { Disagree } \\
\text { Somewhat }\end{array}$ & $\begin{array}{l}\square \\
\text { Neutral }\end{array}$ & $\begin{array}{l}\square \\
\text { Agree } \\
\text { Somewhat }\end{array}$ & $\begin{array}{l}\square \\
\text { Agree }\end{array}$ & $\begin{array}{l}\square \\
\text { Completely } \\
\text { Agree }\end{array}$ \\
\hline
\end{tabular}

4. If their pain would go away, chronic back pain patients would be every bit as active as they used to be.

\begin{tabular}{|l|l|l|l|l|l|l|}
\hline$\square$ & $\square$ & $\square$ & $\square$ & $\square$ & $\square$ & $\square$ \\
$\begin{array}{l}\text { Completely } \\
\text { Disagree }\end{array}$ & $\begin{array}{l}\square \text { Disagree } \\
\text { Somewhat }\end{array}$ & Neutral & $\begin{array}{l}\text { Agree } \\
\text { Somewhat }\end{array}$ & $\begin{array}{l}\square \text { Agree } \\
\text { Completely } \\
\text { Agree }\end{array}$ \\
\hline
\end{tabular}

5. Chronic back pain patients should have the same benefits as the handicapped because of their chronic pain problems.

\begin{tabular}{|l|l|l|l|l|l|l|}
\hline $\begin{array}{l}\text { Completely } \\
\text { Disagree }\end{array}$ & $\begin{array}{l}\square \\
\text { Disagree }\end{array}$ & $\begin{array}{l}\square \\
\text { Disagree } \\
\text { Somewhat }\end{array}$ & $\begin{array}{l}\square \\
\text { Neutral }\end{array}$ & $\begin{array}{l}\square \\
\text { Agree } \\
\text { Somewhat }\end{array}$ & $\begin{array}{l}\square \\
\text { Agree }\end{array}$ & $\begin{array}{l}\square \\
\text { Completely } \\
\text { Agree }\end{array}$ \\
\hline
\end{tabular}

6. Chronic back pain patients owe it to themselves and those around them to perform their usual activities even when their pain is bad. *

\begin{tabular}{|l|l|l|l|l|l|l|}
\hline $\begin{array}{l}\square \\
\text { Completely } \\
\text { Disagree }\end{array}$ & $\begin{array}{l}\square \\
\text { Disagree }\end{array}$ & $\begin{array}{l}\square \\
\text { Disagree } \\
\text { Somewhat }\end{array}$ & $\begin{array}{l}\square \\
\text { Neutral }\end{array}$ & $\begin{array}{l}\square \\
\text { Agree } \\
\text { Somewhat }\end{array}$ & $\begin{array}{l}\square \\
\text { Agree }\end{array}$ & $\begin{array}{l}\square \\
\text { Completely } \\
\text { Agree }\end{array}$ \\
\hline
\end{tabular}

7. Most people expect too much of chronic back pain patients, given their pain.

\begin{tabular}{|l|l|l|l|l|l|l|}
\hline $\begin{array}{l}\text { Completely } \\
\text { Disagree }\end{array}$ & $\begin{array}{l}\square \\
\text { Disagree }\end{array}$ & $\begin{array}{l}\square \\
\text { Disagree } \\
\text { Somewhat }\end{array}$ & $\begin{array}{l}\square \\
\text { Neutral }\end{array}$ & $\begin{array}{l}\square \\
\text { Agree } \\
\text { Somewhat }\end{array}$ & $\begin{array}{l}\square \\
\text { Agree }\end{array}$ & $\begin{array}{l}\square \\
\text { Completely } \\
\text { Agree }\end{array}$ \\
\hline
\end{tabular}

8. Chronic back pain patients have to be careful not to do anything that might make their pain 
worse.

\begin{tabular}{|l|l|l|l|l|l|l|}
\hline$\square$ & $\square$ & $\square$ & $\square$ & $\square$ & $\square$ & $\square$ \\
$\begin{array}{l}\text { Completely } \\
\text { Disagree }\end{array}$ & Disagree & $\begin{array}{l}\square \text { Disagree } \\
\text { Somewhat }\end{array}$ & Neutral & $\begin{array}{l}\text { Agree } \\
\text { Somewhat }\end{array}$ & Agree & $\begin{array}{l}\text { Completely } \\
\text { Agree }\end{array}$ \\
\hline
\end{tabular}

9. As long as they are in pain, chronic back pain patients will never be able to live as well as they did before.

\begin{tabular}{|l|l|l|l|l|l|l|}
\hline $\begin{array}{l}\square \\
\begin{array}{l}\text { Completely } \\
\text { Disagree }\end{array}\end{array}$ & $\begin{array}{l}\square \\
\text { Disagree }\end{array}$ & $\begin{array}{l}\square \\
\text { Disagree } \\
\text { Somewhat }\end{array}$ & $\begin{array}{l}\square \\
\text { Neutral }\end{array}$ & $\begin{array}{l}\square \\
\text { Agree } \\
\text { Somewhat }\end{array}$ & $\begin{array}{l}\square \\
\text { Agree }\end{array}$ & $\begin{array}{l}\square \\
\text { Completely } \\
\text { Agree }\end{array}$ \\
\hline
\end{tabular}

11. Chronic back pain patients have to accept that they are disabled persons, due to their chronic pain.

\begin{tabular}{|l|l|l|l|l|l|l|}
\hline $\begin{array}{l}\square \\
\text { Completely } \\
\text { Disagree }\end{array}$ & $\begin{array}{l}\square \\
\text { Disagree }\end{array}$ & $\begin{array}{l}\square \\
\text { Disagree } \\
\text { Somewhat }\end{array}$ & $\begin{array}{l}\square \\
\text { Neutral }\end{array}$ & $\begin{array}{l}\square \\
\text { Agree } \\
\text { Somewhat }\end{array}$ & $\begin{array}{l}\square \\
\text { Agree }\end{array}$ & $\begin{array}{l}\square \\
\text { Completely } \\
\text { Agree }\end{array}$ \\
\hline
\end{tabular}

12. There is no way that chronic back pain patients can return to doing the things that they used to do, unless they first find a cure for their pain.

\begin{tabular}{|l|l|l|l|l|l|l|}
\hline $\begin{array}{l}\square \\
\begin{array}{l}\text { Completely } \\
\text { Disagree }\end{array}\end{array}$ & $\begin{array}{l}\square \\
\text { Disagree }\end{array}$ & $\begin{array}{l}\square \\
\text { Disagree } \\
\text { Somewhat }\end{array}$ & $\begin{array}{l}\square \\
\text { Neutral }\end{array}$ & $\begin{array}{l}\square \\
\text { Agree } \\
\text { Somewhat }\end{array}$ & $\begin{array}{l}\square \\
\text { Agree }\end{array}$ & $\begin{array}{l}\square \\
\text { Completely } \\
\text { Agree }\end{array}$ \\
\hline
\end{tabular}

14. Even though their pain is always there, chronic back pain patients often don't notice it at all when they keeping themselves busy.

\begin{tabular}{|l|l|l|l|l|l|l|}
\hline $\begin{array}{l}\square \\
\text { Completely } \\
\text { Disagree }\end{array}$ & $\begin{array}{l}\square \\
\text { Disagree }\end{array}$ & $\begin{array}{l}\square \\
\text { Disagree } \\
\text { Somewhat }\end{array}$ & $\begin{array}{l}\square \\
\text { Neutral }\end{array}$ & $\begin{array}{l}\square \\
\text { Agree } \\
\text { Somewhat }\end{array}$ & $\begin{array}{l}\square \\
\text { Agree }\end{array}$ & $\begin{array}{l}\square \\
\text { Completely } \\
\text { Agree }\end{array}$ \\
\hline
\end{tabular}

15. All of the chronic back pain patients' problems would be solved if their pain would go away.

\begin{tabular}{|l|l|l|l|l|l|l|}
\hline $\begin{array}{l}\square \\
\text { Completely } \\
\text { Disagree }\end{array}$ & $\begin{array}{l}\square \\
\text { Disagree }\end{array}$ & $\begin{array}{l}\square \\
\text { Disagree } \\
\text { Somewhat }\end{array}$ & $\begin{array}{l}\square \\
\text { Neutral }\end{array}$ & $\begin{array}{l}\square \\
\text { Agree } \\
\text { Somewhat }\end{array}$ & $\begin{array}{l}\square \\
\text { Agree }\end{array}$ & $\begin{array}{l}\square \\
\text { Completely } \\
\text { Agree }\end{array}$ \\
\hline
\end{tabular}

Scoring: Completely Disagree (1), Disagree (2), Disagree Somewhat (3), Neutral (4), Agree Somewhat (5), Agree (6), Completely Agree (7).

* Items 1,6, and 14 are reverse scored. 


\section{The Pain Attitude and Beliefs Scale For Physical Therapists, Houben ‘05 Version}

Instructions: The purpose of this list is to analyze how you approach the most common forms of back pain. This excludes back pain resulting from a radicular syndrome, cauda equina syndrome, fractures, infections, inflammation, a tumor or metastasis.

1=totally disagree $2=$ largely disagree $3=$ disagree to some extent $4=$ agree to some extent $5=$ largely agree $6=$ totally agree

\begin{tabular}{|c|c|c|}
\hline $6^{+}$ & Mental stress can cause back pain even in the absence of tissue damage & $\begin{array}{lllll}1 & 2 & 3 & 4 & 5\end{array}$ \\
\hline $7^{+}$ & The cause of back pain is unknown & 12345 \\
\hline $10^{*}$ & Pain is a nociceptive stimulus, indicating tissue damage & 123 \\
\hline $11^{\dagger}$ & A patient suffering from severe back pain will benefit from physical exercise & 123345 \\
\hline $12^{+}$ & Functional limitations associated with back pain are the result of psychosocial factors & 123345 \\
\hline $14^{*}$ & Patients with back pain should preferably practice only pain free movements & 12233456 \\
\hline $17^{+}$ & Therapy may have been successful even if pain remains & 123345 \\
\hline $20^{*}$ & Back pain indicates the presence of organic injury & 123345 \\
\hline $22^{*}$ & $\begin{array}{l}\text { If back pain increases in severity, I immediately adjust the intensity of my treatment } \\
\text { accordingly }\end{array}$ & 12345 \\
\hline $23^{*}$ & $\begin{array}{l}\text { If therapy does not result in a reduction in back pain, there is a high risk of severe } \\
\text { restrictions in the long term }\end{array}$ & $\begin{array}{llllll}1 & 2 & 3 & 4 & 5 & 6\end{array}$ \\
\hline $24^{*}$ & Pain reduction is a precondition for the restoration of normal functioning & $\begin{array}{llllll}1 & 2 & 3 & 4 & 5 & 6\end{array}$ \\
\hline $25^{*}$ & Increased pain indicates new tissue damage or the spread of existing damage & 123345 \\
\hline $27^{+}$ & There is no effective treatment to eliminate back pain & 123345 \\
\hline $29^{+}$ & Even if the pain has worsened, the intensity of the next treatment can be increased & 123345 \\
\hline $30^{*}$ & If patients complain of pain during exercise, I worry that damage is being caused & 122345 \\
\hline $31^{*}$ & The severity of tissue damage determines the level of pain & 1223455 \\
\hline $33^{+}$ & Learning to cope with stress promotes recovery from back pain & 12233456 \\
\hline $34^{+}$ & Exercises that may be back straining should not be avoided during treatment & 12345 \\
\hline $35^{*}$ & $\begin{array}{l}\text { In the long run, patients with back pain have a higher risk of developing spinal } \\
\text { impairments }\end{array}$ & $\begin{array}{llllll}1 & 2 & 3 & 4 & 5 & 6\end{array}$ \\
\hline
\end{tabular}

${ }^{*}=$ biomedical subscale, $+=$ biopsychosocial subscale 


\section{Neurophysiology of Pain Questionnaire}

$\begin{array}{lll}\mathbf{T} & \mathbf{U}\end{array}$

\begin{tabular}{|l|l|l|l|}
\hline 1 & It is possible to have pain and not know about it & & \\
\hline 2 & $\begin{array}{l}\text { When part of your body is injured, special pain receptors convey the pain message to } \\
\text { your brain }\end{array}$ & & \\
\hline 3 & Pain only occurs when you are injured or at risk of being injured & & \\
\hline 4 & $\begin{array}{l}\text { When you are injured, special receptors convey the danger message to your spinal } \\
\text { cord }\end{array}$ & & \\
\hline 5 & Special nerves in your spinal cord convey 'danger' messages to your brain & & \\
\hline 6 & Nerves adapt by increasing their resting level of excitement & & \\
\hline 7 & Chronic pain means that an injury hasn't healed properly & & \\
\hline 8 & The body tells the brain when it is in pain & & \\
\hline 9 & Nerves adapt by making ion channels stay open longer & & \\
\hline 10 & Descending neurons are always inhibitory & & \\
\hline 11 & Pain occurs whenever you are injured & $\begin{array}{l}\text { When you injure yourself, the environment that you are in will not affect the amount } \\
\text { of pain you experience, as long as the injury is exactly the same }\end{array}$ & \\
\hline 12 & The brain decides when you will experience pain & \\
\hline 13 & T & & \\
\hline
\end{tabular}

$\mathrm{T}=$ True, $\mathrm{F}=$ False, $\mathrm{U}=$ Undecided 


\section{References}

1. Hoy D, Bain C, Williams G, et al. A systematic review of the global prevalence of low back pain. Arthritis Rheum. 2012;64(6):2028-2037.

2. Hoy D, March L, Brooks $P$, et al. The global burden of low back pain: Estimates from the global burden of disease 2010 study. Ann Rheum Dis. 2014;73(6):968-974.

3. Jarvik JG, Hollingworth W, Heagerty PJ, Haynor DR, Boyko EJ, Deyo RA. Three-year incidence of low back pain in an initially asymptomatic cohort: Clinical and imaging risk factors. Spine. 2005;30(13):1541-1549.

4. Brinjikji W, Luetmer PH, Comstock B, et al. Systematic literature review of imaging features of spinal degeneration in asymptomatic populations. AJNR Am J Neuroradiol. 2015;36(4):811-816.

5. Christensen S, Hartvigsen J. Spinal curves and health: A systematic critical review of the epidemiological literature dealing with associations between sagittal spinal curves and health. $J$ Man Manip Therap. 2008;31(9):690-714.

6. Saragiotto BT, Maher CG, Yamato TP, et al. Motor control exercise for chronic non-specific low-back pain. Cochrane Database Syst Rev. 2016;CD012004(1).

7. Adams N, Poole H, Richardson C. Psychological approaches to chronic pain management: Part 1. J Clin Nurs. 2006;15(3):290-300.

8. George SZ, Fritz JM, McNeil DW. Fear-avoidance beliefs as measured by the fear-avoidance beliefs questionnaire: Change in fear-avoidance beliefs questionnaire is predictive of change in self-report of disability and pain intensity for patients with acute low back pain. Clin J Pain. 2006;22(2):197-203.

9. Lakke SE, Soer R, Krijnen WP, van der Schans CP, Reneman MF, Geertzen JH. Influence of physical therapists' kinesiophobic beliefs on lifting capacity in healthy adults. Phys Ther. 2015;95(9):1224-1233.

10. Rainville J, Bagnall D, Phalen L. Health care providers' attitudes and beliefs about functional impairments and chronic back pain. Clin J Pain. 1995;11(4):287-295.

11. Ostelo RW, Stomp-van den Berg SG, Vlaeyen JW, Wolters PM, de Vet HC. Health care provider's attitudes and beliefs towards chronic low back pain: The development of a questionnaire. Man Ther. 2003;8(4):214-222.

12. Balague F, Mannion AF, Pellise F, Cedraschi C. Non-specific low back pain. Lancet. 2012;379(9814):482-491. 
13. Walker BF, Muller R, Grant WD. Low back pain in australian adults: Prevalence and associated disability. J Manipulative Physiol Ther. 2004;27(4):238-244.

14. Walker BF. The prevalence of low back pain: A systematic review of the literature from 1966 to 1998. J Spinal Disord. 2000;13(3):205-217.

15. Meucci RD, Fassa AG, Faria NM. Prevalence of chronic low back pain: Systematic review. Rev Saude Publica. 2015;49(1).

16. Johannes CB, Le TK, Zhou X, Johnston JA, Dworkin RH. The prevalence of chronic pain in united states adults: Results of an internet-based survey. J Pain. 2010;11(11):1230-1239.

17. Katz JN. Lumbar disc disorders and low-back pain: Socioeconomic factors and consequences. J Bone Joint Surg Am. 2006;88 Suppl 2:21-24.

18. Jette AM, Smith K, Haley SM, Davis KD. Physical therapy episodes of care for patients with low back pain. Phys Ther. 1994;74(2):101-10; discussion 110-5.

19. Dagenais S, Caro J, Haldeman S. A systematic review of low back pain cost of illness studies in the united states and internationally. Spine J. 2008;8(1):8-20.

20. Engel G. The need for a new medical model: A challenge for biomedicine. Science. 1977;196:129-136.

21. Gatchel RJ, Turk DC. Criticisms of the biopsychosocial model in spine care: Creating and then attacking a straw person. Spine. 2008;33(25):2831-2836.

22. Sharp TJ. Chronic pain: A reformulation of the cognitive-behavioural model. Behav Res Ther. 2001;39(7):787-800.

23. Leeuw M, Goossens ME, Linton SJ, Crombez G, Boersma K, Vlaeyen JW. The fear-avoidance model of musculoskeletal pain: Current state of scientific evidence. J Behav Med. 2007;30(1):77-94.

24. Vlaeyen JW, Linton SJ. Fear-avoidance and its consequences in chronic musculoskeletal pain: A state of the art. Pain. 2000;85(3):317-332.

25. Carragee EJ, Alamin TF, Miller JL, Carragee JM. Discographic, MRI and psychosocial determinants of low back pain disability and remission: A prospective study in subjects with benign persistent back pain. Spine J. 2005;5(1):24-35.

26. Linton SJ. A review of psychological risk factors in back and neck pain. Spine. 2000;25(9):1148-1156. 
27. Linton SJ, Buer N, Vlaeyen J, Hellsing AL. Are fear-avoidance beliefs related to the inception of an episode of back pain? A prospective study. Psychol Health. 2000;14(6):1051-1059.

28. Klenerman L, Slade PD, Stanley IM, et al. The prediction of chronicity in patients with an acute attack of low back pain in a general practice setting. Spine. 1995;20(4):478-484.

29. Pincus T, Burton AK, Vogel S, Field AP. A systematic review of psychological factors as predictors of chronicity/disability in prospective cohorts of low back pain. Spine.

2002;27(5):109-120.

30. Picavet HS, Vlaeyen JW, Schouten JS. Pain catastrophizing and kinesiophobia: Predictors of chronic low back pain. Am J Epidemiol. 2002;156(11):1028-1034.

31. Wertli M, Rasmussen-Barr E, Weiser S, Bachmann LM, Brunner F. The role of fear avoidance beliefs as a prognostic factor for outcome in patients with nonspecific low back pain: $A$ systematic review. Spine J. 2014;14(5):816-836.

32. Wertli MM, Eugster R, Held U, Steurer J, KofmehI R, Weiser S. Catastrophizing-a prognostic factor for outcome in patients with low back pain: A systematic review. Spine J. 2014;14(11):2639-2657.

33. Thomas EN, Pers YM, Mercier G, et al. The importance of fear, beliefs, catastrophizing and kinesiophobia in chronic low back pain rehabilitation. Ann Phys Rehabil Med. 2010;53(1):3-14.

34. Sions JM, Hicks GE. Fear-avoidance beliefs are associated with disability in older american adults with low back pain. Phys Ther. 2011;91(4):525-534.

35. Lundberg M, Frennered K, Hagg O, Styf J. The impact of fear-avoidance model variables on disability in patients with specific or nonspecific chronic low back pain. Spine.

2011;36(19):1547-1553.

36. Woby SR, Watson PJ, Roach NK, Urmston M. Are changes in fear-avoidance beliefs, catastrophizing, and appraisals of control, predictive of changes in chronic low back pain and disability? Eur J Pain. 2004;8(3):201-210.

37. Flynn T, Fritz J, Whitman J, et al. A clinical prediction rule for classifying patients with low back pain who demonstrate short-term improvement with spinal manipulation. Spine.

2002;27:2835-2843.

38. Childs JD, Fritz JM, Flynn TW, et al. A clinical prediction rule to identify patients with low back pain most likely to benefit from spinal manipulation: A validation study. Ann Intern Med. 2004;141(12):920-928. 
39. Smeets RJ, Vlaeyen JW, Kester AD, Knottnerus JA. Reduction of pain catastrophizing mediates the outcome of both physical and cognitive-behavioral treatment in chronic low back pain. J Pain. 2006; 7(4):261-271.

40. Feitosa AS, Lopes JB, Bonfa E, Halpern AS. A prospective study predicting the outcome of chronic low back pain and physical therapy: The role of fear-avoidance beliefs and extraspinal pain. Rev Bras Reumatol Engl Ed. 2016;56(5):384-390.

41. Cleland J, Fritz J, Brennan G. Predictive validity of initial fear avoidance beliefs in patients with low back pain receiving physical therapy: Is the FABQ a useful screening tool for identifying patients at risk for a poor recovery. Eur Spine J. 2008;17(1):70-79.

42. Louw A, Zimney K, Puentedura EJ, Diener I. The efficacy of pain neuroscience education on musculoskeletal pain: A systematic review of the literature. Physiother Theory Pract. 2016;32(5):332-355.

43. Linton SJ, Vlaeyen J, Ostelo R. The back pain beliefs of health care providers: Are we fearavoidant? J Occup Rehabil. 2002;12(4):223-232.

44. Overmeer T, Boersma K, Main CJ, Linton SJ. Do physical therapists change their beliefs, attitudes, knowledge, skills and behaviour after a biopsychosocially orientated university course? J Eval Clin Pract. 2009;15(4):724-732.

45. Simmonds MJ, Derghazarian T, Vlaeyen JW. Physiotherapists' knowledge, attitudes, and intolerance of uncertainty influence decision making in low back pain. Clin J Pain. 2012;28(6):467-474.

46. Houben RM, Ostelo RW, Vlaeyen JW, Wolters PM, Peters M, Stomp-van den Berg SG. Health care providers' orientations towards common low back pain predict perceived harmfulness of physical activities and recommendations regarding return to normal activity. Eur J Pain. 2005;9(2):173-183.

47. Daykin AR, Richardson B. Physiotherapists' pain beliefs and their influence on the management of patients with chronic low back pain. Spine. 2004;29(7):783-795.

48. Bishop A, Foster N, Thomas E, Hay E. How does the self-reported clinical management of patients with low back pain relate to the attitudes and beliefs of health care practitioners? A survey of UK general practitioners and physiotherapists. Pain. 2008;135:187-195.

49. Coste J, Lefrancois G, Guillemin F, Pouchot J, French Study Group for Quality of Life in Rheumatology. Prognosis and quality of life in patients with acute low back pain: Insights from a comprehensive inception cohort study. Arthritis Rheum. 2004;51(2):168-176. 
50. Pengel LH, Herbert RD, Maher CG, Refshauge KM. Acute low back pain: Systematic review of its prognosis. BMJ. 2003;327(7410):323.

51. Von Korff M, Deyo RA, Cherkin D, Barlow W. Back pain in primary care. outcomes at 1 year. Spine. 1993;18(7):855-862.

52. Maher CG. Effective physical treatment for chronic low back pain. Orthop Clin North Am. 2004;35(1):57-64.

53. van Middelkoop M, Rubinstein SM, Kuijpers $T$, et al. A systematic review on the effectiveness of physical and rehabilitation interventions for chronic non-specific low back pain. Eur Spine J. 2011;20(1):19-39.

54. Rainville J, Carlson N, Polatin P, Gatchel RJ, Indahl A. Exploration of physicians' recommendations for activities in chronic low back pain. Spine. 2000;25(17):2210-2220.

55. Darlow B, Fullen B, Dean S, Hurley D, Baxter G, Dowell A. The association between health care professional attitudes and beliefs and the attitudes and beliefs, clinical management, and outcomes of patients. Eur J Pain. 2012;16(1):3-17.

56. Darlow B, Dowell A, Baxter GD, Mathieson F, Perry M, Dean S. The enduring impact of what clinicians say to people with low back pain. Ann Fam Med. 2013;11(6):527-534.

57. Darlow B. Beliefs about back pain: The confluence of client, clinician and community. Int J Osteopath MED. 2016;20:53-61.

58. Darlow B, Dean S, Perry M, Mathieson F, Baxter G, Dowell A. Easy to harm, hard to heal:Patient views about the back. Spine. 2015;40(11):842-850.

59. Wertli M, Rasmussen-Barr E, Held U, Weiser S, Bachmann LM, Brunner F. Fear-avoidance beliefs-a moderator of treatment efficacy in patients with low back pain: A systematic review. Spine J. 2014;14(11):2658-2678.

60. Park SJ, Yoon DM, Yoon KB, Moon JA, Kim SH. Factors associated with higher reported pain levels in patients with chronic musculoskeletal pain: A cross-sectional, correlational analysis. PLoS One. 2016;11(9):e0163132.

61. Houben RM, Vlaeyen JW, Peters M, Ostelo RW, Wolters PM, Stomp-van den Berg SG. Health care providers' attitudes and beliefs towards common low back pain: Factor structure and psychometric properties of the HC-PAIRS. Clin J Pain. 2004;20(1):37-44.

62. Bishop A, Thomas E, Foster NE. Health care practitioners' attitudes and beliefs about low back pain: A systematic search and critical review of available measurement tools. Pain. 2007;132(1-2):91-101. 
63. Fullen BM, Baxter GD, Doody C, Daly LE, Hurley DA. General practitioners' attitudes and beliefs regarding the management of chronic low back pain in ireland: A cross-sectional national survey. Clin J Pain. 2011;27(6):542-549.

64. Delitto A, George SZ, Van Dillen LR, et al. Low back pain clinical practice guideline. J Orthop Sports Phys Ther. 2012;42(4):1-57.

65. Rosenthal R, Reed L. A longitudinal study of the effects of experimenter bias on the operant learning of laboratory rats. J Psychiat Res. 1963;2:61-77.

66. Galer BS, Schwartz L, Turner JA. Do patient and physician expectations predict response to pain-relieving procedures? Clin J Pain. 1997;13(4):348-351.

67. IASP terminology. International Association for the Study of Pain Web site. https://www.iasp-pain.org/terminology?\&navltemNumber=576. Updated 2017. Accessed May 21, 2018.

68. van Tulder $M$, Becker $A$, Bekkering $T$, et al. European guidelines for the management of acute nonspecific low back pain in primary care. Eur Spine J. 2006;15 Suppl 2:S169-91.

69. Fritz JM, Brennan GP, Clifford SN, Hunter SJ, Thackeray A. An examination of the reliability of a classification algorithm for subgrouping patients with low back pain. Spine. 2006;31:77-82.

70. Delitto A, Erhard RE, Bowling RW. A treatment-based classification approach to low back syndrome: Identifying and staging patients for conservative treatment. Phys Ther. 1995;75:470485.

71. Qaseem A, Wilt TJ, McLean RM, Forciea MA, Clinical Guidelines Committee of the American College of Physicians. Noninvasive treatments for acute, subacute, and chronic low back pain: $A$ clinical practice guideline from the american college of physicians. Ann Intern Med. 2017;166(7):514-530.

72. Manchikanti L. Epidemiology of low back pain. Pain Physician. 2000;3(2):167-192.

73. Vos T, Allen C, Arora M, Barber R, Bhutta Z, Brown A. Global, regional, and national incidence, prevalence, and years lived with disability for 310 diseases and injuries, 1990-2015: A systematic analysis for the global burden of disease study 2015. Lancet. 2016;388(10053):15451602.

74. Deyo RA, Tsui-Wu YJ. Descriptive epidemiology of low-back pain and its related medical care in the united states. Spine. 1987;12(3):264-268.

75. Sternbach R. Survey of pain in the united states: The nuprin pain report. Clin J Pain. 1986:49-53. 
76. Lawrence RC, Helmick CG, Arnett FC, et al. Estimates of the prevalence of arthritis and selected musculoskeletal disorders in the united states. Arthritis Rheum. 1998;41(5):778-799.

77. Rubin DI. Epidemiology and risk factors for spine pain. Neurol Clin. 2007;25(2):353-371.

78. Waddell G. 1987 volvo award in clinical sciences. A new clinical model for the treatment of low-back pain. Spine. 1987;12(7):632-644.

79. Snodgrass SJ, Haskins R, Rivett DA. A structured review of spinal stiffness as a kinesiological outcome of manipulation: Its measurement and utility in diagnosis, prognosis and treatment decision-making. J Electromyogr Kinesiol. 2012;22(5):708-723.

80. Gilbert FJ, Grant AM, Gillan MG, et al. Does early imaging influence management and improve outcome in patients with low back pain? A pragmatic randomized controlled trial. Health Technol Assess. 2004;8(17):1-131.

81. Fordyce W. A behavioral perspective on chronic pain. Br J Clin Psychol. 1982;21:313-320.

82. Fordyce M, Fowler R, Lehmann J, DaLateur B. Some implications of learning in problems of chronic pain. J Chron Dis. 1968;21:179-190.

83. Fordyce $W$, Roberts $A$, Sternbach $R$. The behavioral management of chronic pain: $A$ response to critics. Spine. 1985;22:113-125.

84. Fordyce M, Shelton J, Dundore D. The modification of avoidance learning pain behaviors. J Behav Med. 1982;5:405-414.

85. Pincus T, Kent $P$, Bronfort G, Loisel P, Pransky G, Harbigsen J. Twenty-five years with the biopsychosocial model of low back pain-is it time to celebrate? Spine. 2013;38(24):2118-2123.

86. Chou R, Qaseem A, Snow V, et al. Diagnosis and treatment of low back pain: A joint clinical practice guideline from the american college of physicians and the american pain society. Ann Intern Med. 2007;147(7):478-491.

87. Mariano TY, Urman RD, Hutchison CA, Jamison RN, Edwards RR. Cognitive behavioral therapy (CBT) for subacute low back pain: A systematic review. Curr Pain Headache Rep. 2018;22(3):15-018-0669-5.

88. George SZ, Wittmer VT, Fillingim RB, Robinson ME. Comparison of graded exercise and graded exposure clinical outcomes for patients with chronic low back pain. J Orthop Sports Phys Ther. 2010;40(11):694-704.

89. Lethem J, Slade PD, Troup JD, Bentley G. Outline of a fear-avoidance model of exaggerated pain perception--I. Behav Res Ther. 1983;21(4):401-408. 
90. Neblett R. Pain catastrophizing: An historical perspective. J Appl Behav Res. 2017;22(12086).

91. Sullivan MJ, Thorn B, Haythornthwaite JA, et al. Theoretical perspectives on the relation between catastrophizing and pain. Clin J Pain. 2001;17(1):52-64.

92. Sullivan M. The pain catastrophizing scale user manual. http://sullivanpainresearch.mcgill.ca/pdf/pcs/PCSManual English.pdf;. Updated 2009. Accessed May 15, 2018.

93. Grotle M, Vollestad NK, Brox JI. Clinical course and impact of fear-avoidance beliefs in low back pain: Prospective cohort study of acute and chronic low back pain. Spine. 2006;31(9):10381046.

94. Sullivan MJ, Stanish W, Waite H, Sullivan M, Tripp DA. Catastrophizing, pain, and disability in patients with soft-tissue injuries. Pain. 1998;77(3):253-260.

95. Mallen CD, Peat G, Thomas E, Dunn KM, Croft PR. Prognostic factors for musculoskeletal pain in primary care: A systematic review. Br J Gen Pract. 2007;57(541):655-661.

96. Al-Obaidi SM, Beattie P, Al-Zoabi B, Al-Wekeel S. The relationship of anticipated pain and fear avoidance beliefs to outcome in patients with chronic low back pain who are not receiving workers' compensation. Spine. 2005;30(9):1051-1057.

97. Swinkels-Meewisse IE, Roelofs J, Verbeek AL, Oostendorp RA, Vlaeyen JW. Fear-avoidance beliefs, disability, and participation in workers and non-workers with acute low back pain. Clin J Pain. 2006;22(1):45-54.

98. Fritz JM, George SZ. Identifying psychosocial variables in patients with acute work-related low back pain: The importance of fear-avoidance beliefs. Phys Ther. 2002;82(10):973-983.

99. Grotle M, Vollestad NK, Veierod MB, Brox JI. Fear-avoidance beliefs and distress in relation to disability in acute and chronic low back pain. Pain. 2004;112(3):343-352.

100. Mannion AF, Junge A, Taimela S, Muntener M, Lorenzo K, Dvorak J. Active therapy for chronic low back pain: Part 3. factors influencing self-rated disability and its change following therapy. Spine. 2001;26(8):920-929.

101. Severeijns R, Vlaeyen JW, van den Hout MA, Weber WE. Pain catastrophizing predicts pain intensity, disability, and psychological distress independent of the level of physical impairment. Clin J Pain. 2001;17(2):165-172.

102. Turner JA, Jensen MP, Warms CA, Cardenas DD. Catastrophizing is associated with pain intensity, psychological distress, and pain-related disability among individuals with chronic pain after spinal cord injury. Pain. 2002;98(1-2):127-134. 
103. Westman AE, Boersma K, Leppert J, Linton SJ. Fear-avoidance beliefs, catastrophizing, and distress: A longitudinal subgroup analysis on patients with musculoskeletal pain. Clin J Pain. 2011;27(7):567-577.

104. Swinkels-Meewisse IE, Roelofs J, Oostendorp RA, Verbeek AL, Vlaeyen JW. Acute low back pain: Pain-related fear and pain catastrophizing influence physical performance and perceived disability. Pain. 2006;120(1-2):36-43.

105. Peters ML, Vlaeyen JW, Weber WE. The joint contribution of physical pathology, painrelated fear and catastrophizing to chronic back pain disability. Pain. 2005;113(1-2):45-50.

106. George SZ, Stryker SE. Fear-avoidance beliefs and clinical outcomes for patients seeking outpatient physical therapy for musculoskeletal pain conditions. J Orthop Sports Phys Ther. 2011;41(4):249-259.

107. Sullivan M, Tripp D, Santor D. Gender differences in pain and pain behavior: The role of catastrophizing. Cognit Ther Res. 2000;24(1):121-134.

108. Boersma K, Linton SJ. How does persistent pain develop? an analysis of the relationship between psychological variables, pain and function across stages of chronicity. Behav Res Ther. 2005;43(11):1495-1507.

109. Fritz JM, George SZ, Delitto A. The role of fear-avoidance beliefs in acute low back pain: Relationships with current and future disability and work status. Pain. 2001;94(1):7-15.

110. Jensen JN, Albertsen K, Borg V, Nabe-Nielsen K. The predictive effect of fear-avoidance beliefs on low back pain among newly qualified health care workers with and without previous low back pain: A prospective cohort study. BMC Musculoskelet Disord. 2009;10:117-2474-10117.

111. Nicholas MK, Linton SJ, Watson PJ, Main CJ, "Decade of the Flags" Working Group. Early identification and management of psychological risk factors ("yellow flags") in patients with low back pain: A reappraisal. Phys Ther. 2011;91(5):737-753.

112. Pincus T, Vogel S, Burton AK, Santos R, Field AP. Fear avoidance and prognosis in back pain: A systematic review and synthesis of current evidence. Arthritis Rheum. 2006;54(12):39994010.

113. Underwood MR, Morton V, Farrin A, UK BEAM Trial Team. Do baseline characteristics predict response to treatment for low back pain? secondary analysis of the UK BEAM dataset. Rheumatology. 2007;46(8):1297-1302.

114. Vowles KE, Gross RT. Work-related beliefs about injury and physical capability for work in individuals with chronic pain. Pain. 2003;101(3):291-298. 
115. Bergbom S, Boersma K, Overmeer T, Linton SJ. Relationship among pain catastrophizing, depressed mood, and outcomes across physical therapy treatments. Phys Ther. 2011;91(5):754764.

116. Wertli MM, Burgstaller JM, Weiser S, Steurer J, Kofmehl R, Held U. Influence of catastrophizing on treatment outcome in patients with nonspecific low back pain: A systematic review. Spine. 2014;39(3):263-273.

117. van Tulder MW, Ostelo R, Vlaeyen JW, Linton SJ, Morley SJ, Assendelft WJ. Behavioral treatment for chronic low back pain: A systematic review within the framework of the cochrane back review group. Spine. 2000;25(20):2688-2699.

118. Moseley GL. Evidence for a direct relationship between cognitive and physical change during an education intervention in people with chronic low back pain. Eur J Pain. 2004;8:39-45.

119. Moseley GL, Butler DS. Fifteen years of explaining pain: The past, present, and future. $J$ Pain. 2015;16(9):807-813.

120. Robinson V, King R, Ryan C, Martin D. A qualitative exploration of people's experiences of pain neurophysiological education for chronic pain: The importance of relevance for the individual. Man Ther. 2016;22:56-61.

121. Qaseem A, Wilt TJ, McLean RM, Forciea MA, Clinical Guidelines Committee of the American College of Physicians. Noninvasive treatments for acute, subacute, and chronic low back pain: A clinical practice guideline from the american college of physicians. Ann Intern Med. 2017;166(7):514-530.

122. Richmond H, Hall AM, Copsey B, et al. The effectiveness of cognitive behavioural treatment for non-specific low back pain: A systematic review and meta-analysis. PLoS One. 2015;10(8):e0134192.

123. Moseley L. Combined physiotherapy and education is efficacious for chronic low back pain. Aust J Physiother. 2002;48:297-302.

124. Malfliet A, Kregel J, Coppieters I, et al. Effect of pain neuroscience education combined with cognition-targeted motor control training on chronic spinal pain: A randomized clinical trial. JAMA Neurol. 2018.

125. Ryan CG, Gray HG, Newton M, Granat MH. Pain biology education and exercise classes compared to pain biology education alone for individuals with chronic low back pain: A pilot randomized controlled trial. Man Ther. 2010;15(4):382-387. 
126. Van Oosterwijck J, Meeus M, Paul L, et al. Pain physiology education improves health status and endogenous pain inhibition in fibromyalgia: A double-blind randomized controlled trial. Clin J Pain. 2013;29(10):873-882.

127. Vibe Fersum K, O'Sullivan P, Skouen JS, Smith A, Kvale A. Efficacy of classification-based cognitive functional therapy in patients with non-specific chronic low back pain: A randomized controlled trial. Eur J Pain. 2013;17(6):916-928.

128. Beltran-Alacreu H, Lopez-de-Uralde-Villanueva I, Fernandez-Carnero J, La Touche R. Manual therapy, therapeutic patient education, and therapeutic exercise, an effective multimodal treatment of nonspecific chronic neck pain: A randomized controlled trial. Am J Phys Med Rehabil. 2015;94(10):887-889.

129. Gallagher L, McAuley J, Moseley GL. A randomized-controlled trial of using a book of metaphors to reconceptualize pain and decrease catastrophizing in people with chronic pain. Clin J Pain. 2013;29(1):20-25.

130. Meeus M, Nijs J, Van Oosterwijck J, Van Alsenoy V, Truijen S. Pain physiology education improves pain beliefs in patients with chronic fatigue syndrome compared with pacing and selfmanagement education: A double-blind randomized controlled trial. Arch Phys Med Rehabil. 2010;91(8):1153-1159.

131. Moseley GL, Nicholas MK, Hodges PW. A randomized controlled trial of intensive neurophysiology education in chronic low back pain. Clin J Pain. 2004;20(5):324-330.

132. Louw A, Diener I, Landers MR, Puentedura EJ. Preoperative pain neuroscience education for lumbar radiculopathy: A multicenter randomized controlled trial with 1-year follow-up. Spine. 2014;39(18):1449-1457.

133. Tegner H, Frederiksen P, Esbensen BA, Juhl C. Neurophysiological pain education for patients with chronic low back pain: A systematic review and meta-analysis. Clin J Pain. 2018;34(8):778-786.

134. Traeger AC, Lee $\mathrm{H}$, Hubscher $\mathrm{M}$, et al. Effect of intensive patient education vs placebo patient education on outcomes in patients with acute low back pain: A randomized clinical trial. JAMA Neurol. 2019;76(2):161-169.

135. Gardner T, Refshauge K, Smith L, McAuley J, Hübscher M, Goodall S. Physiotherapists' beliefs and attitudes influence clinical practice in chronic low back pain: A systematic review of quantitative and qualitative studies. Journal of Physiotherapy. 2017;63(3):132-143.

136. Ostelo RW, Stomp-van den Berg SG, Vlaeyen JW, Wolters PM, de Vet HC. Health care provider's attitudes and beliefs towards chronic low back pain: The development of a questionnaire. Man Ther. 2003;8(4):214-222. 
137. Nitesh B, Chhabra S. Attitudes \& beliefs of physiotherapists towards low back pain: A review of literature. Indian J Physiother Occup Ther. 2016;10(1):151-156.

138. Houben RM, Gijsen A, Peterson J, de Jong PJ, Vlaeyen JW. Do health care providers' attitudes towards back pain predict their treatment recommendations? differential predictive validity of implicit and explicit attitude measures. Pain. 2005;114(3):491-498.

139. Nolan D, O'Sullivan K, Stephenson J, O'Sullivan P, Lucock M. What do physiotherapists and manual handling advisors consider the safest lifting posture, and do back beliefs influence their choice? Musculoskelet Sci Pract. 2018;33:35-40.

140. Jeffrey JE, Foster NE. A qualitative investigation of physical therapists' experiences and feelings of managing patients with nonspecific low back pain. Phys Ther. 2012;92(2):266-278.

141. Mackey L, Hurley D. A comparison of physiotherapy students' and recently graduated physiotherapists' attitudes and beliefs to patients with chronic low back pain (CLBP) in ireland A pilot study. PHYSIOTHER PRACT RES. 2014;35(2):101-109.

142. Coudeyre E, Rannou F, Tubach F, et al. General practitioners' fear-avoidance beliefs influence their management of patients with low back pain. Pain. 2006;124(3):330-337.

143. Epstein-Sher S, Jaffe DH, Lahad A. Are they complying? physicians' knowledge, attitudes, and readiness to change regarding low back pain treatment guideline adherence. Spine. 2017;42(4):247-252.

144. Poiraudeau S, Rannou F, Baron G, et al. Fear-avoidance beliefs about back pain in patients with subacute low back pain. Pain. 2006;124(3):305-311.

145. Askew R, Kibelstis C, Overbaugh S, Walker S, Nixon-Cave K, Shepard KF. Physical therapists' perception of patients' pain and its effect on management. Physiother Res Int. 1998;3(1):37-57.

146. Derghazarian T, Simmonds MJ. Management of low back pain by physical therapists in quebec: How are we doing? Physiother Can. 2011;63(4):464-473.

147. Valjakka AL, Salanterä S, Laitila A, Julkunen J, Hagelberg NM. The association between physicians' attitudes to psychosocial aspects of low back pain and reported clinical behaviour: $A$ complex issue. Scandinavian Journal of Pain. 2013;4(1):25-30.

148. Sieben JM, Vlaeyen JW, Portegijs PJ, et al. General practitioners' treatment orientations towards low back pain: Influence on treatment behaviour and patient outcome. Eur J Pain. 2009;13(4):412-418.

149. Bishop A, Foster NE. Do physical therapists in the united kingdom recognize psychosocial factors in patients with acute low back pain? Spine. 2005;30(11):1316-1322. 
150. Laekeman MA, Sitter H, Basler HD. The pain attitudes and beliefs scale for physiotherapists: Psychometric properties of the german version. Clin Rehabil. 2008;22(6):564575.

151. Watson PJ, Bowey J, Purcell-Jones G, Gales T. General practitioner sickness absence certification for low back pain is not directly associated with beliefs about back pain. Eur J Pain. 2008;12(3):314-320.

152. Mclntosh A, Shaw CF. Barriers to patient information provision in primary care: Patients' and general practitioners' experiences and expectations of information for low back pain. Health Expect. 2003;6(1):19-29.

153. Josephson I, Hedberg B, Bulow P. Problem-solving in physiotherapy--physiotherapists' talk about encounters with patients with non-specific low back pain. Disabil Rehabil. 2013;35(8):668-677.

154. Werner EL, Ihlebaek C, Skouen JS, Laerum E. Beliefs about low back pain in the norwegian general population: Are they related to pain experiences and health professionals? Spine. 2005;30(15):1770-1776.

155. Overmeer T, Boersma K. What messages do patients remember? relationships among patients' perceptions of physical therapists' messages, patient characteristics, satisfaction, and outcome. Phys Ther. 2016;96(3):275-283.

156. Coudeyre E, Tubach F, Rannou F, et al. Fear-avoidance beliefs about back pain in patients with acute LBP. Clin J Pain. 2007;23(8):720-725.

157. Witt CM, Martins F, Willich SN, Schutzler L. Can I help you? physicians' expectations as predictor for treatment outcome. Eur J Pain. 2012;16(10):1455-1466.

158. Ambady N, Koo J, Rosenthal R, Winograd CH. Physical therapists' nonverbal communication predicts geriatric patients' health outcomes. Psychol Aging. 2002;17(3):443452.

159. Birkhauer J, Gaab J, Kossowsky J, et al. Trust in the health care professional and health outcome: A meta-analysis. PLoS One. 2017;12(2):e0170988.

160. Bialosky JE, Bishop MD, Cleland JA. Individual expectation: An overlooked, but pertinent, factor in the treatment of individuals experiencing musculoskeletal pain. Phys Ther.

2010;90(9):1345-1355.

161. Gregg CD, McIntosh G, Hall H, Hoffman CW. Prognostic factors associated with low back pain outcomes. J Prim Health Care. 2014;6(1):23-30. 
162. Campbell P, Foster NE, Thomas E, Dunn KM. Prognostic indicators of low back pain in primary care: Five-year prospective study. J Pain. 2013;14(8):873-883.

163. Overmeer T, Boersma K, Denison E, Linton SJ. Does teaching physical therapists to deliver a biopsychosocial treatment program result in better patient outcomes? A randomized controlled trial. Phys Ther. 2011;91(5):804-819.

164. Beneciuk J, George S. Pragmatic implementation of a stratified primary care model for low back pain management in outpatient physical therapy settings: Two-phase, sequential preliminary study. Phys Ther. 2015;95(8):1120-34.

165. Vonk F, Pool JJ, Ostelo RW, Verhagen AP. Physiotherapists' treatment approach towards neck pain and the influence of a behavioural graded activity training: An exploratory study. Man Ther. 2009;14(2):131-137.

166. Abdel Shaheed C, Graves J, Maher C. The effects of a brief educational intervention on medical students' knowledge, attitudes and beliefs towards low back pain. Scandinavian Journal of Pain. 2017;16:101-104.

167. Ryan C, Murphy D, Clark M, Lee A. The effect of a physiotherapy education compared with a non-healthcare education on the attitudes and beliefs of students towards functioning in individuals with back pain: An observational, cross-sectional study. Physiotherapy. 2010;96(2):144-150.

168. Cox T, Louw A, Puentedura EJ. An abbreviated therapeutic neuroscience education session improves pain knowledge in first-year physical therapy students but does not change attitudes or beliefs. J Man Manip Therap. 2017;25(1):11-21.

169. Cleland JA, Fritz JM, Brennan GP, Magel J. Does continuing education improve physical therapists' effectiveness in treating neck pain? A randomized clinical trial. Phys Ther. 2009;89(1):38-47.

170. Pincus T, Foster NE, Vogel S, Santos R, Breen A, Underwood M. Attitudes to back pain amongst musculoskeletal practitioners: A comparison of professional groups and practice settings using the ABS-mp. Man Ther. 2007;12(2):167-175.

171. Magalhaes MO, Costa LO, Cabral CM, Machado LA. Attitudes and beliefs of brazilian physical therapists about chronic low back pain: A cross-sectional study. Rev Bras Fisioter. 2012;16(3):248-253.

172. Ferreira PH, Ferreira ML, Latimer J, et al. Attitudes and beliefs of brazilian and australian physiotherapy students towards chronic back pain: A cross-cultural comparison. Physiother Res Int. 2004;9(1):13-23. 
173. Alshami A, Albahrani Y. A comparison of the attitudes toward chronic low back pain in saudi, australian and brazilian physical therapy students. J Taibah Univ Sci. 2015;10(2):181-87.

174. Catley MJ, O'Connell NE, Moseley GL. How good is the neurophysiology of pain questionnaire? A rasch analysis of psychometric properties. J Pain. 2013;14(8):818-827.

175. Watson AW, Mac Donncha C. A reliable technique for the assessment of posture: Assessment criteria for aspects of posture. J Sports Med Phys Fitness. 2000;40(3):260-270.

176. Domenech J, Segura-Orti E, Lison JF, Espejo-Tort B, Sanchez-Zuriaga D. Psychometric properties and factor structure of the spanish version of the HC-PAIRS questionnaire. Eur Spine J. 2013;22(5):985-994.

177. Riley JF, Ahern DK, Follick MJ. Chronic pain and functional impairment: Assessing beliefs about their relationship. Arch Phys Med Rehabil. 1988;69(8):579-582.

178. Magalhaes MO, Costa LO, Ferreira ML, Machado LA. Clinimetric testing of two instruments that measure attitudes and beliefs of health care providers about chronic low back pain. Rev Bras Fisioter. 2011;15(3):249-256.

179. Moran RW, Rushworth WM, Mason J. Investigation of four self-report instruments (FABT, TSK-HC, back-PAQ, HC-PAIRS) to measure healthcare practitioners' attitudes and beliefs toward low back pain: Reliability, convergent validity and survey of new zealand osteopaths and manipulative physiotherapists. Musculoskeletal Science and Practice. 2017;32:44-50.

180. Evans D, Breen A, Pincus T, et al. The effectiveness of a posted information package on the beliefs and behavior of musculoskeletal practitioners: The UK chiropractors, osteopaths, and musculoskeletal physiotherapists low back pain management (COMPLeMENT) randomized trial. Spine. 2010;35(8):858-866.

181. Portney L, Watkins M. Foundations of clinical research application to practice. 3rd ed. Philadelphia, PA: F.A. Davis Company; 2015:892.

182. Tavakol M, Dennick R. Making sense of cronbach's alpha. Int J Med Educ. 2011;2:53-55.

183. Koo TK, Li MY. A guideline of selecting and reporting intraclass correlation coefficients for reliability research. J Chiropr Med. 2016;15(2):155-163.

184. McManus I. The misinterpretation of the standard error of measurement in medical education: A primer on the problems, pitfalls and peculiarities of the three different standard errors of measurment. Med Teach. 2012;34(7):569-76.

185. Latimer J, Maher C, Refshauge K. The attitudes and beliefs of physiotherapy students to chronic back pain. Clin J Pain. 2004;20(1):45-50. 
186. Slater H, Briggs A, Smith A, Bunzli S, Davies S, Quinter J. Implementing evidence-informed policy into practice for health care professionals managing people with low back pain in australian rural settings: A preliminary prospective single-cohort study. Pain Med. 2018;15(10):1657-1658.

187. Colleary G, O'Sullivan K, Griffin D, Ryan CG, Martin DJ. Effect of pain neurophysiology education on physiotherapy students' understanding of chronic pain, clinical recommendations and attitudes towards people with chronic pain: A randomised controlled trial. Physiotherapy (United Kingdom). 2017;103(4):423-429.

188. Morris H, Ryan C, Lauchlan D, Field M. Do medical student attitudes towards patients with chronic low back pain improve during training? a cross-sectional study. BMC Med Educ. 2012;12:10-6920-12-10.

189. Beneciuk JM, Bishop MD, George SZ. Clinical prediction rules for physical therapy interventions: A systematic review. Phys Ther. 2009;89:114-124.

190. Davis J. Construct validity in measurement: A pattern matching approach. Eval Program Plann. 1989;12(1):31-36.

191. Eland ND, Kvale A, Ostelo RW, Strand LI. The pain attitudes and beliefs scale for physiotherapists: Dimensionality and internal consistency of the norwegian version. Physiother Res Int. 2016.

192. Bowey-Morris J, Purcell-Jones G, Watson PJ. Test-retest reliability of the pain attitudes and beliefs scale and sensitivity to change in a general practitioner population. Clin J Pain. 2010;26(2):144-152.

193. Moseley L. Unraveling the barriers to reconceptualization of the problem in chronic pain: The actual and perceived ability of patients and health professionals to understand the neurophysiology. J Pain. 2003;4(4):184-189.

194. Demoulin C, Brasseur P, Roussel N, et al. Cross-cultural translation, validity, and reliability of the french version of the neurophysiology of pain questionnaire. Physiother Theory Pract. 2017;33(11):880-887.

195. Meeus M, Niks J, Elsemans K, Truijen S, De Meirleis K. Development and properties of the dutch neuro-physiology of pain test in patients with chronic fatigue syndrome. J Musculoskelet Pain. 2010;18:58-65.

196. Lee H, McAuley JH, Hubscher M, Kamper SJ, Traeger AC, Moseley GL. Does changing painrelated knowledge reduce pain and improve function through changes in catastrophizing? Pain. 2016;157(4):922-930. 
197. Adillon C, Lozano E, Salvat I. Comparison of pain neurophysiology knowledge among health sciences students: A cross-sectional study. BMC Res Notes. 2015;8:592-015-1585-y.

198. Louw A, Diener I, Puentedura EJ. The short term effects of preoperative neuroscience education for lumbar radiculopathy: A case series. Int J Spine Surg. 2015;9:11.

199. Focus on therapeutic outcomes INC. http://www.fotoinc.com/. Accessed September 12, 2018.

200. Stratford PW, Binkley JM, Riddle DL. Development and initial validation of the back pain functional scale. Spine (Phila Pa 1976). 2000;25(16):2095-2102.

201. Hart DL, Werneke MW, Wang YC, Stratford PW, Mioduski JE. Computerized adaptive test for patients with lumbar spine impairments produced valid and responsive measures of function. Spine. 2010;35(24):2157-2164.

202. Focus on therapeutic outcome Inc. Test-retest reliablity of FOTO functional status outcomes surveys. http://cdn2.hubspot.net/hub/442011/file-1970004530-pdf/docs/NQF TestRetest Reliability Assessment Report.pdf?t=1432064040387. Updated 2013. Accessed September 15, 2018.

203. Hart DL, Stratford PW, Werneke MW, Deutscher D, Wang YC. Lumbar computerized adaptive test and modified oswestry low back pain disability questionnaire: Relative validity and important change. J Orthop Sports Phys Ther. 2012;42(6):541-551.

204. Wang YC, Hart DL, Werneke M, Stratford PW, Mioduski JE. Clinical interpretation of outcome measures generated from a lumbar computerized adaptive test. Phys Ther. 2010;90(9):1323-1335.

205. Deutscher D, Werneke MW, Hayes D, et al. Impact of risk adjustment on provider ranking for patients with low back pain receiving physical therapy. J Orthop Sports Phys Ther. 2018;48(8):637-648.

206. Brooks G, Dolphin M, Vanbeveren P, Hart DL. Referral source and outcomes of physical therapy care in patients with low back pain. J Orthop Sports Phys Ther. 2012;42(8):705-715.

207. Gozalo PL, Resnik $\sqcup$, Silver B. Benchmarking outpatient rehabilitation clinics using functional status outcomes. Health Serv Res. 2016;51(2):768-789.

208. Rodeghero JR, Cook CE, Cleland JA, Mintken PE. Risk stratification of patients with low back pain seen in physical therapy practice. Man Ther. 2015;20(6):855-860.

209. Resnik L, Liu D, Mor V, Hart DL. Predictors of physical therapy clinic performance in the treatment of patients with low back pain syndromes. Phys Ther. 2008;88(9):989-1004. 
210. Resnik L, Hart DL. Using clinical outcomes to identify expert physical therapists. . 2003;83:990-1002.

211. Burgess R, Bishop A, Lewis M, Hill J. Models used for case-mix adjustment of patient reported outcome measures (PROMs) in musculoskeletal healthcare: A systematic review of the literature. Physiotherapy. 2019;105(2):137-146.

212. Risk adjustment \& predictive analytics. Focus on Therapeutic Outcomes, Inc Web site. https://www.fotoinc.com/risk-adjustment-predictive-analysis. Accessed December,11, 2019.

213. Waddell G, Newton M, Henderson I, Somerville D, Main CJ. A fear-avoidance beliefs questionnaire (FABQ) and the role of fear-avoidance beliefs in chronic low back pain and disability. Pain. 1993;52(2):157-168.

214. Grotle M, Brox JI, Vollestad NK. Reliability, validity and responsiveness of the fearavoidance beliefs questionnaire: Methodological aspects of the norwegian version. $J$ Rehabil Med. 2006;38(6):346-353.

215. Pfingsten $M$, Kroner-Herwig B, Leibing E, Kronshage $U$, Hildebrandt J. Validation of the german version of the fear-avoidance beliefs questionnaire (FABQ). Eur J Pain. 2000;4(3):259266.

216. Chaory K, Fayad F, Rannou F, et al. Validation of the french version of the fear avoidance belief questionnaire. Spine. 2004;29(8):908-913.

217. Kovacs FM, Muriel A, Medina JM, et al. Psychometric characteristics of the spanish version of the FAB questionnaire. Spine. 2006;31(1):104-110.

218. Swinkels-Meewisse EJ, Swinkels RA, Verbeek AL, Vlaeyen JW, Oostendorp RA. Psychometric properties of the tampa scale for kinesiophobia and the fear-avoidance beliefs questionnaire in acute low back pain. Man Ther. 2003;8(1):29-36.

219. Ostelo RW, Swinkels-Meewisse IJ, Knol DL, Vlaeyen JW, de Vet HC. Assessing pain and pain-related fear in acute low back pain: What is the smallest detectable change? Int J Behav Med. 2007;14(4):242-248.

220. Chung EJ, Hur YG, Lee BH. A study of the relationship among fear-avoidance beliefs, pain and disability index in patients with low back pain. J Exerc Rehabil. 2013;9(6):532-535.

221. Cai C, Pua YH, Lim KC. Correlates of self-reported disability in patients with low back pain: The role of fear-avoidance beliefs. Ann Acad Med Singapore. 2007;36(12):1013-1020. 
222. George SZ, Valencia C, Beneciuk JM. A psychometric investigation of fear-avoidance model measures in patients with chronic low back pain. J Orthop Sports Phys Ther. 2010;40(4):197205.

223. Meyers L, Gamst G, Guarino A. Applied multivariate research design and interpretation. second ed. California, USA: Sage Publication INC; 2013:1078.

224. Hoaglin D, Iglewicz B. Fine tuning some resistant rules for outlier labeling. J Am Stat Assoc. 1987;82:1147-1149.

225. Feng $\mathrm{C}$, Wang $\mathrm{H}$, Lu N, et al. Log-transformation and its implications for data analysis. Shanghai Arch Psychiatry. 2014;26(2):105-109.

226. Ruengvirayudh $\mathrm{P}$, Brooks $\mathrm{G}$. Comparing stepwise regression models to the best-subsets models, or, the arto of stepwise. Gen Linear Mod J. 2016;42(1):1-14.

227. Zhang Z. Variable selection with stepwise and best subset approaches. Ann Transl Med. 2016;4(7):136.

228. Li W, Nyholt DR. Marker selection by akaike information criterion and bayesian information criterion. Genet Epidemiol. 2001;21 Suppl 1:S272-7.

229. Courtney C, Fernandez-de-Las-Penas C, Bond S. Mechanisms of chronic pain - key considerations for appropriate physical therapy management. The Journal of manual \& manipulative therapy. 2017;25(3):118-127.

230. Gatchel RJ, Neblett R, Kishino N, Ray CT. Fear-avoidance beliefs and chronic pain. J Orthop Sports Phys Ther. 2016;46(2):38-43.

231. Louw A, Puentedura E. Therapeutic neuroscience education. OPTP; 2013:292.

232. Domenech J, Sanchez-Zuriaga D, Segura-Orti E, Espejo-Tort B, Lison JF. Impact of biomedical and biopsychosocial training sessions on the attitudes, beliefs, and recommendations of health care providers about low back pain: A randomised clinical trial. Pain. 2011;152(11):2557-2563.

233. Monnin D, Courvoisier DS, Genevay S. Modifying beliefs about back pain: A pilot study among healthcare professionals. Patient Educ Couns. 2016;99(4):665-670.

234. Slater H, Davies SJ, Parsons R, Quintner JL, Schug SA. A policy-into-practice intervention to increase the uptake of evidence-based management of low back pain in primary care: $A$ prospective cohort study. PLoS One. 2012;7(5):e38037. 
235. Slater H, Briggs A, Smith A, Bunzli S, Davies S, Quintner J. Implementing evidence-informed policy into practice for health care professionals managing people with low back pain in australian rural settings: A preliminary prospective single cohort study. Pain Med.

2014;15(10):1657-1658.

236. Schiltenwolf $M$, Buchner $M$, Heindl B, von Reumont J, MÃ $1 / 1$ ller A, Eich W. Comparison of a biopsychosocial therapy (BT) with a conventional biomedical therapy (MT) of subacute low back pain in the first episode of sick leave: A randomized controlled trial. European spine journal : official publication of the European Spine Society, the European Spinal Deformity Society, and the European Section of the Cervical Spine Research Society. 2006;15(7):1083-1092.

237. Hancock MJ, FAU MC, Laslett MF, Hay EF, Koes B. Discussion paper: What happened to the 'bio' in the bio-psycho-social model of low back pain? Eur Spine J. 2011;20(12):2105-10.

238. George SZ, Zeppieri G,Jr, Cere AL, et al. A randomized trial of behavioral physical therapy interventions for acute and sub-acute low back pain (NCT00373867). Pain. 2008;140(1):145157.

239. Beneciuk JM, Robinson ME, George SZ. Low back pain subgroups using fear-avoidance model measures: Results of a cluster analysis. Clin J Pain. 2012;28(8):658-666. 